\title{
Theoretical and Numerical Studies of Constitutive Relations for Frictional Granular Flow
}

\author{
Final Report
}

Donald D. Gray

James M. Stiles

Ismail Celik

January 1991

Work Performed Under Contract No.: DE-FC21-87MC24207

For

U.S. Department of Energy

Office of Fossil Energy

Morgantown Energy Technology Center

Morgantown, West Virginia

By

West Virginia University

Department of Civil Engineering

Morgantown, West Virginia 


\section{DISCLAIMER}

This report was prepared as an account of work sponsored by an agency of the United States Government. Neither the United States Government nor any agency thereof, nor any of their employees makes any warranty, express or implied, or assumes any legal liability or responsibility for the accuracy, completeness or usefulness of any information, apparatus, product, or process disclosed, or represents that its use would not infringe privately owned rights. Reference herein to any specific commercial product, process, or service by trade name, trademark, manufacturer, or otherwise, does not necessaril'; constitute or imply its endorsement, recommendation, or favoring by the United States Government or any agency thereof. The views and opinions of authors expressed herein do not necessarily state or reflect those of the United States Government or any agency thereof.

This report has been reproduced directly from the best available copy.

Available to DOE and DOE contractors from the Office of Scientific and Technical Information, P.O. Box 62, Oak Ridge, TN 37831; prices available from (615)576-8401, FऽS 626-8401.

Available to the public from the National Technical Information Service, U.S. Department of Commerce, 5285 Port Royal Rd., Springfield, VA 22161. 


\title{
Theoretical and Numerical Studies of Constitutive Relations for Frictional Granular Flow
}

\author{
Final Report
}

\author{
Donald D. Gray \\ James M. Stiles \\ Ismail Celik
}

Work Performed Under Contract No.: DE-FC21-87MC24207

\author{
For \\ U.S. Department of Energy \\ Office of Fossil Energy \\ Morgantown Energy Technology Center \\ P.O. Box 880 \\ Morgantown, West Virginia 26507-0880 \\ By \\ West Virginia University \\ Department of Civil Engineering \\ Morgantown, West Virginia 26506
}

Ji.luary 1991 


\section{TABLE OF CONTENTS}

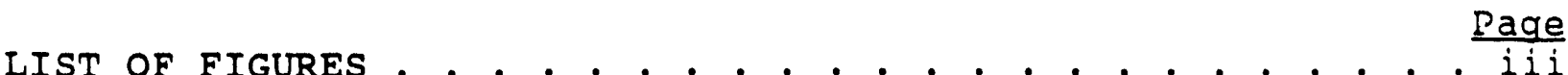
LIST OF TABLES . . . . . . . . . . . . . . . . . vi vi 1.0 INTRODUCTION . . . . . . . . . . . . . . . . 1 1.1 Literature Review . . . . . . . . . . . . . . 2

2.0 CONSTITUTIVE RELATIONSHIPS FOR FRICTIONAL FLOW . . . . . . 8

3.0 CODING THE GRAY-STILES CONSTITUTIVE RELATIONSHIP • • . . 19 3.1 Evaluation of the Material Properties . . . . . . . 22 3.2 Results Using the Gray-Stiles Constitutive Relationship . . . . . . . . 25

4.0 CODING THE VON MISES CONSTITUTIVE RELATIONSHIP . . . . . 27

4.1 Results Using the Von Mises Constitutive Relationship . . . . . . . . 28

5.0 CONCLUSIONS . . . . . . . . . . . . . . . 36

6.0 ACKNOWLEDGEMENT . . . . . . . . . . . . . . . 37

7.0 REFERENCES . . . . . . . . . . . . . . . . . . 38

8.0 FIGURES . . . . . . . . . . . . . . . . . . . . 41

APPENDIX 1. GENERALIZATION OF THE GRAY-STILES .......80

APPENDIX 2. GRANULAR FLOW EQUATIONS FOR SPECIAL
CASES AND THEIR SOLUTIONS . . . . . . . . . . 90

APPENDIX 3. MODIFIED VERSION OF TEACH FOR
THE VON MISES SIMULATIONS . . . . . . . . . . . . 96 


\section{LIST OF FIGURES}

FIGURE NO.

PAGE

Sign Convention

Von Mises Yield Surface . . . . . . . . . 43

Associated Flow Rule . . . . . . . . . 44

Extended Von Mises Yield Surface . . . . . . 45

Mohr-Coulomb Yield Surface . . . . . . . 46

Gray-Siles Yield Surface . . . . . . . . 47

Gray-Stiles Yield surface and

Associated Flow Rule . . . . . . . . . 48

Schematic of a Shear cell from

Cernica (1982) . . . . . . . . . . . . . . 49

Example Plot of Failure Envelope and Corresponding Mohr Circle . . . . . . 50

Schematic of standard Compressibility

Tester Erom Royal (1986) . . . . . . . . 51

Critical state Pressure Versus Density on Logarithmic Axes for Granular

Material No. 1 . . . . . . . . . . 52

2 Critical state Pressure Versus Density

on Logarithmic Axes for Granular

Material No. 2 . . . . . . . . . . 53

Critical state Pressure Versus Density

on Logarithmic Axes for Granular

Material No. 3 . . . . . . . . . . 54

Critical state Pressure Versus Density

on Logarithmic Axes for Granular

Material No. 4 . . . . . . . . . . 55

Critical State Pressure Versus Density

on Logarithmic Axes for Granular

Material No. 5 . . . . . . . . . . 56

Critical state Pressure Versus Density

on Logarithmic Axes for Granular

Material No. 6 . . . . . . . . . . 57

Finite Difference Grid Used for the

Von Mises Material Simulations . . . . . 58 
19 Pressure Contour Plot for the Von Mises Simulation after 500 Iterations . . . . . 60 Simulation after 10,000 Iterations . . . . 63

Pressure Contour Plot for the Von Mises Simulation after 10,000 Iterations . . . . 64

Velocity Profiles for Newtonian PoiseuilleCouette Simulation after 1000 Iterations . . 65

Midline Pressure for Newtonian PoiseuilleCouette Simulation after 1000 Iterations. . . 66

Velocity Profiles for Newtonian/Von Mises/ Inviscid Poiseuille-Couette Simulation . . . 67

Midline Pressure for Newtonian/Von Mises/ Inviscid Poiseuille-Couette Simulation . . . 68

Velocity Profiles for Newtonian/Von Mises/ Bounded Viscosity Poiseuille-Couette Simulation .. . . . . . . . . . . . 69

Midline Pressure for Newtonian/Von Mises/ Bounded Viscosity Poiseuille-Couette Simulation................. . 70

30 Velocity Profiles for Newtonian/Von Mises/ Bounded Viscosity Plus Kinetic Effects Poiseuille-Couette Simulation . . . . . . 71

31 Midline Pressure for Newtonian/Von Mises/ Bounded Viscosity Plus Kinetic Effects Poiseuille-Couette Simulation . . . . . . 72 Features of Haigh-Westergaard Space . . . . 73

33 Distinction between Convexity and Dissipativeriess . . . . . . . . . . . 74

34 Gray-stiles Yield surface for Cohesive Material...... . . . . . . . . . 75 
Dimensionless Radius of Yield Surface

4 for $n=0.5,1.0,2.0$. . . . . . . 76

36 Yield Surface 4 in Haigh-Westergaard

space with $p=3, \sigma_{0}=-1$,

$\mathrm{k}=0.4837, \mathrm{n}=0.2$. . . . . . . . . . 77

Dimensionless Radius of Yield Surface

5 for $\mathrm{m}=0.5,1.0,2.0 . . . . . . . .78$

38 Yield Surface 5 in Haigh-Westergaard

Space with $\mathrm{p}=3, \sigma_{1}=-1$,

$\mathrm{k}=0.4837, \mathrm{~m}=0.2^{\prime \prime} . . . . . . . . . .79$ 


\subsection{INTRODUCTION}

Some of the most important processes in modern industry involve the flow of granular materials. Unfortunately the flow of granular materials is poorly understood. Although granular flow was studied as early as 1852 by Hagen, only in recent years has the need for precise information concerning the flow of granular materials been recognized by industry. This need for precise knowledge has been brought about by the automation of process equipment which must handle flowing granular materials (Brown and Richards, 1970).

In the energy industry, coal is by far the most important granular material. One of the best ways to burn coal for electricity generation is in a fluidized bed. Pulverized coal in a fluidized bed flows in one of three basic regimes: fluidized two-phase flow, rapid unfluidized flow, and frictional unfluidized flow. Fluidized two-phase flow occurs when the coal particles are influenced dynamically by drag stresses due to the interstitial fluid. Rapid unfluidized flow occurs when only the collisional stresses between particles are dynamically important. Frictional unfluidized flow is present when both collisional and drag stresses are insignificant. This lack of collisions results in essentially continuous inter-particle contact which induces frictional stresses between the particles in a flowing granular material.

With the appropriate constitutive relationship, one can apply the continuum assumption to derive and solve the conservation equations of mass and momentum for any of the three regimes. A suitable constitutive relationship for the fluidized two-phase regime has been proposed by syamlal (1985). This constitutive relationship has also been incorporated successfully in a finite difference computer program (MFIX) which numerically solves the equations of momentum and continuity for both the gas and the solid phases. MFIX uses a separate solid phase for each particle size and density present in the flow in order to model phenomena such as segregation and elutriation (Syamlal, 1987). A suitable constitutive relationship for the rapid unfluidized regime based upon the principles of the kinetic theory of gases has also been developed (Boyle, Massoudi, and O'Brien, 1988). Likewise this constitutive relationship has been successfully implemented in the computer program MFIX (Syamlal, 1988). No suitable constitutive relationship has yet been accepted for the frictional flow regime.

The purpose of this report is to propose a suitable constitutive relationship for the three dimensional frictional flow of a cohesionless granular material and to incorporate at least a qualitatively similar constitutive relationship in the computer program TEACH. TEACH is a hydrodynamic code developed by Dr. A.D. Gosman at Imperial College, London, for the simulation of incompressible two dimensional steady duct flows. Simulations performed for this report assumed that the material was flowing under two dimensional plane strain conditions. The numerical 
algorithm implemented by TEACH is based upon the control volume finite difference principles developed by spalding and Patankar (Patankar, 1980). TEACH's program structure facilitates the implementation of a non-Newtonian constitutive relationship of the type proposed below.

\subsection{Literature Review}

Three approaches have been used to study the problem of frictional granular flow: discrete particle, micromechanical, and phenomeological. The discrete particle approach does not make the continuum assumption for the granular material, but models the flow as an assemblage of discrete spherical particles by numerically solving the conservation equations of linear and angular momentum for each particle. The micromechanical approach views the granular material as a continuum, but bases the constitutive relationship only on the microscopic behavior and properties of the solid particles which make up the granular material. The phenomenological approach also makes the continuum assumption, but the constitutive relationship is based solely on the macroscopic behavior and properties of the material.

The most successful method used in the discrete particle approach is the distinct element method developed by cundall and strack (1979). The distinct element method calculates the equilibrium contact forces and displacements of a stressed assembly of particles with a series of calculations that trace the movements of individual particles. The individual particle movements result from the propagation of disturbances that originate at the boundaries of the assembly of particles. Time steps are taken over which the velocities and accelerations of the particles can be assumed constant. This assumption is valid when the time steps are small enough that disturbances cannot propagate from any particle farther than its immediate neighbors. The resultant force on any particle can be determined exclusively by its interaction with the particles it comes in contact with. Although the distinct element method is completely explicit, computer memory and computational time requirements severely limit the number of particles one can model; only 1500 two dimensional particles can be modeled with the in-core memory of a minicomputer with $64 \mathrm{~K}$ of 32 -bit words (Cundall and Strack, 1979). Walton, Braun, and Cervelli (1986) extended the method to solve for assemblies of three dimensional pirticles. With the three dimensional computer models, they studied the rheology of assemblies of inelastic, frictional pariicles in steady shearing flow. While the results of the discrete particle approach have been promising, this approach is impractical even with today's supercomputers.

The micromechanical approach is based upon the fact that the macroscopic constitutive relationship is a function only of those quantities that are related to the microstructure of the granular material. The microstructure of a granular material is defined by 
the placement of solid particles and void spaces in the granular material. The two basic properties of the microstructure are the coordination number (number of contacts per particle) and the void fraction. The basic problem with the micromechanical approach is the assumptions needed to average the microscopic properties to formulate a macroscopic constitutive relationship (Mehrabadi, 1986).

Like the micromechanical approach, the phenomenological approach makes the continuum assumption for the granular material. The phenomenological approach on the other hand, attempts to create a constitutive relationship based solely upon macroscopic properties. Because the microstructure of the granular material is ignored, the phenomenological approach is necessarily an approximate one. Most of the work performed from this approach has been based upon the theory of plasticity.

The theory of plasticity contains two basic hypotheses: the yield condition and the flow rule. The yield condition determines whether or not plastic deformations will occur, and the flow rule governs the direction of those plastic deformations. Granular materials are often assumed to be rigid-plastic materials, which is to say that the material undergoes no deformation until the loading satisfies the yield condition. Similarly elastic-plastic materials undergo only elastic deformations before the yield condition is satisfied. In 1776, Coulomb proposed the first yield condition for granular materials (Erown and Richards, 1970). This yield condition is a linear relationship between applied normal stress and shear stress at yield. The sole purpose of coulomb's work was to determine what set of circumstances would cause a soil to yield. In order to derive a momentum equation for the yielding granular material one must also postulate a flow rule.

Drucker and Prager (1952) were the first to apply the principles of plasticity to granular flow. The yield condition they proposed was a balance of the hydrostatic stress and the square root of the second invariant of the deviatoric stress tensor. They also proposed the associated flow rule as the stress-strain relation. The associated flow rule assumes that the principal deformation rate vector is an outward normal to the yield surface when the principal deformation rates are plotted on the same axes as the principal stresses. While Drucker and Prager's goal was to develop stability theorems for soil mechanics problems, they were the first to provide a basis from which one could derive a momentum equation for the flow of granular materials. Their model predicts both a positive definito dilatation rate and a zero rate of energy dissipation. These two attributes are unacceptable for a model describing the frictional flow of granular materials.

Jenike, Elsey, and Woolley (1960) proposed three modifications to the Mohr-Coulomb yield condition to permit the yield condition to reflect changes in the granular material caused by consolidation: 
1. For small values of applied normal stress, the yield condition becomes curved.

2. The yield surface does not extend indefinitely with increasing normal stress but terminates at a point $\mathrm{E}$.

3. The position of terminal point $E$ is not a constant but varies with the bulk density of the granular material.

This modified Mohr-Coulomb yield surface was applied to the flow of bulk solids using the alignment flow rule (Jenike, 1961). The alignment flow rule states that the eigenvectors of the deformation rate tensor and the deviatoric stress tensor are identical. This formulation suffers from analytical difficulties due to the shape of the yield surface in Haigh-Westergaard space and possesses severe problems with ill-posedness (Pitman, 1989). HaighWestergaard space is a space spanned by the principal stresses in which every conceivable state of stress is represented by a point.

Schofield and wroth (1968) described a new concept of the yielding of granular materials which was first proposed by Roscoe, Schofield, and wroth in 1958. This critical state hypothesis states that as the granular material yields, it approaches the critical state by either dilatating or consolidating (Schofield and Wroth, 1968).

One of the first models for the deformation of granular materials based upon the critical state concept was the Granta-gravel model. Granta-gravel is a hypothetical cohesionless volumetric strain hardening, rigid-plastic material with positive energy dissipation. When shown in Haigh-Westergaard space, the yield surface is a teardrop-shaped surface of revolution about the hydrostatic axis. The Granta-gravel model agrees with most of the experimental results presented by schofield and wroth except for tests of undrained granular materials yielding in the critical state. This deficiency is attributed by schofield and wroth to the lack of elastic strains in the model. Schofield and Wroth present the Granta-gravel model using stress parameters from triaxial testing. This presentatior makes the physical meaning and the generalization to three dimensions obscure. Although these problems and the analytical difficulties with its yield surface have hindered the widespread usage of the Granta-gravel model in soil mechanics, the critical state concept itself has become the dominant theory of frictional granular flow.

In a review article in 1983, Jackson reviewed plasticity and critical state theory and described attempts to solve the problem of incompressible granular material discharge from a cone. The governing equations were the two dimensional conservation equations of momentum and mass and a constitutive relationship that consisted of an associated flow rule and a convex yield surface. The discharge problem was treated as a steady state incompressible 
problem near the exit boundary of the hopper. The boundary conditions included traction-free surfaces at the exit boundary and at the upper free surface and a frictional condition based upon Coulomb's law at the walls. The theoretical predictions were shown to disagree with experimental results of Sullivan. The discrepancy between theory and experiment was attributed by Jackson to the assumptions of incompressibility and of a traction-free surface at the exit boundary of the hopper. Photographic evidence by Bosley et al in 1969 was presented which indicated that the exjt boundaries may be better approximated by a surface below which the particles fall vertically under the influence of gravity alone. While simulations performed with this boundary condition agreed better with the experimental results, the problem of the exit boundary condition was by no means resolved. From Jackson's results it is clear that the effects of compressibility, collisional stresses, boundary conditions, and the intergranular fluid on the discharge problem remain to be determined.

In 1987, schaeffer investiyated the linear instability of the evolution equations describing incompressible granular flow. The constitutive model he used consisted of the yield condition first proposed by Drucker and Prager (1952) and a nonassociated flow rule based upon critical state ideas which insured positive energy dissipation. The main result was the discovery of violent instability due to linear ill-posedness analogous to the backwards heat equation for parameter values arising in most physically realistic situations. From these results and the experimental fact that the granular flow in discharging silos is typically pulsating, Schaeffer conjectured that there are time periodic solutions of the elastic-plastic equations governing frictional granular flow. While this conjecture appears to be reasonable, the analytical and numerical difficulties with the elastic-plastic equations prevent the testing of his conjecture.

Pitman and schaeffer, in 1987, examined the stability of compressible granular flow under two dimensional plane strain conditions. The constitutive relationship used by Pitman and Schaeffer was of the same form as the Granta-gravel model proposed by Schofield and wroth (1968) and consisted of a convex yield surface and the associated flow rule. Pitmin and Schaeffer's linear stability analysis indicates that most of the $i 11$-posedress described by schaeffer $(1987)$ is damped by the inclusion of compressibility effects. The remaining ill-posedness is not damped by the inclusion of elastic effects, but only occurs wheil the granular material is dilatating.

In 1988, Schaeffer and Pitman examined the question of wellposedness for the three dimensional compressible flow of granular materials with the governing equations derived in their earlier publication (Pitman and Schaeffer, 1987). The central conclusion of their work was that the governing equations are linearly wellposed as long as the flow is fully three dimensional. In flow that 
is fully three dimensional all three principal deformation rates are nonzero. As one of the principal deformation rates goes to zero, ill-posedness may appear, as described in their earlier paper (Pitman and Schaeffer, 1987). While the possession of linear well or ill-posedness carries no rigorous implications for the nonlinear theory, these results indicate that some of the phenomena observed in two dimensinnal plane strain experiments may not be present in fully three dimensional flow. Unfortunately most of the experimental work in grardalar flow is condicted under plane stiain conditions.

Schaeffer, Shearer, and Pitman, in 4988 , investigatec the linear stability of the governing equations of two dimensional compressible granular flow. Unlike their previous publications, this paper deals with linear instability in the more customary sense, that is, under what conditions does the linear theory predict that small deviations from a homogeneous deformation wili grow exponentially with time. The main conclusion of their work was that the boundary between stable and unstable flow conditions is on the consolidation side of the yield surface when the flow is two dimensional. Other observations made were that the steady state equations are elliptic when the flow is stable and hyperbolic when the flow is unstable, and that all of the principal stresses are compressive. A numerical algorithm solving the unsteady equations would have to adjust to the changes in the mathematical type of the steady state equations (Pitman, 1989).

Pitman and schaeffer, in a review article written in 1988, discussed the $r$ previous work concerning the problems of the stability and Fusedness of the governing equations of granular flow and presented the results of attempts to solve the governing equations for granular flow in a shear cell. Pitman and schaeffer conjectured that the formation of shear bands in the granular material is a consequence of the transition of the governing equations from stable to unstable and then to ill-posed. In the proximity of a shear band there is the transfer of a large amount of momentum over distances on the order of the particle diameter. In this region the frictional granular constitutive relationship is not realistic, but the system can be regularized by using a constitutive relationship for the rapid flow regime such as was developed by Jenkins and Savage (1983). This was accomplished by assuming that the total granular stress tensor is equal to the sum of the critical state theory stress tensor and the kinetic theory stress tensor. When the granular material is deforming slowly and uniformly, the contribution of the kinetic theory stress tensor is negligible and the critical state stress tensor predominates. on the other hand, near the location of a shear band the critical state stress tensor is negligible and the kinetic theory predominates. The numerical results presented were in approximate agreement with experimental observations of shear band formation in flowing granular materials. 
Gray and stiles (1988) proposed a constitutive relationship for three dimensional cumpressible granular flow based upon the two dimensional constitutive relationship used by Pitman and schaeffer (1987). Gray and stiles's analysis shows that the constitutive relationship allows dilatation and consolidation and possesses a positive definite plastic dissipation rate. The stability and posedness of the governing equations with the proposed constitutive relationship is described by Pitman and schaeffer (1988).

Baxter, Behringer, Faggert, and Johnson (1989) report the results of experiments involving the gravity flow of faceted and smooth sand through a wedge-shaped hopper. These experiments observed spatial variations in density using digital subtraction radiography. The objectives of these experiments were to establish the spatial patterns of density waves in granular flow tlirough a wedge-shaped hopper, look for the existence of bifurcations, and to compare existing predictions for fully developed granular flow. The experiments indicate that density waver form and propagate in faceted sand and mixtures of smooth and faceted sand. The formation and propagation of these waves are controlled by various factors such as the mass flow rate, hopper angle, and the inass fraction of the faceted sand. The results also indicate the existence of a bifurcation when the mass fraction of the faceted sand is approximately 0.2. Jenike's (1961) solutions indicated that there should be a transition between uniform flow and funnel flow, but the experimental results do not show any transition. Funnel flow is hopper flow that possesses an interface between flowing and stagnant regions. 


\subsection{CONSTITUTIVE RELATIONSHIPS FOR FRICTIONAL' FLOW}

As was mentioned in the introduction, the constitutive relationship for the frictional flow of granular materials presented here will be based upon the priniiples of plasticity, a subject which has developed primarily to describe the behavior of ductile metals. A compressive sign convention has been chosen for the sticess and deformation rate tensors because cohesionless granular materials cannot resist tension. The sign convention is shown in Figure 1. The two basic hypotheses of plasticity are the yield condition and the flow rule. The yield condition indivates the onset of plastic behavior and is represented by a surface in Haigh-Westergaard space. The axes in Haigh-Westergaard space represent the principal stresses, so that every possible state of stress is represented by a point. The yield condition for ductile metals is unaffected by the mean normal stress, $\langle\sigma\rangle$. This fact forces the yield surface to be a cylinder whose axis parallels the hydrostatic axis. The hydrostatic axis is the line that passes through the roints $(0,0,0)$ and $(1,1,1)$ in Haigh-Westergaard space. The axis of the yield surface of an isotropic material will coincide with the hydrostatic axis because the yield surface must be unaffected by a relabeling of the coordinate axis.

The simplest yield surfaces possessing these attributes are the hexagonal and circular cylinders. The Tresca yield condition, the hexagonal cylinder, can be viewed as a limitation on the maximum shear stress. The Tresca yield surface will not be discussed further in this report because of the analytical difficulties with its sharp corners.

The von Mises yield condition for ductile metals, the circular cylinder, is interpreted as being a restraint on the distortional strain energy. The von Mises yield surface is shown in Figure 2 . Mathematicaliy speaking, the von Mises yield condition is a limitation on the second invariant of the deviatoric stress tensor, $J_{2 D}$, and is given by the function $F_{1}$.

$$
F_{1}=-J_{2 D}-k^{2}=0
$$

$J_{2 D}$ is defined by equation (2).

$$
J_{2 D}=-\frac{1}{2} \sum_{i=1}^{3}\left(\sigma_{i}-\langle\sigma\rangle\right)^{2}
$$

where:

$$
<\sigma\rangle=\left(\sigma_{11}+\sigma_{22}+\sigma_{33}\right) / 3=\left(\sigma_{1}+\sigma_{2}+\sigma_{3}\right) / 3
$$


The single subscript in (2) indicates that the principal stress vector is indicated.

$J_{2 D}$ car be expressed in the following ways.

$$
\begin{aligned}
J_{2 D}= & -\frac{1}{6}\left[\left(\sigma_{1}-\sigma_{2}\right)^{2}+\left(\sigma_{2}-\sigma_{3}\right)^{2}+\left(\sigma_{3}-\sigma_{1} j^{2}\right]\right. \\
= & -\frac{1}{6}\left[\left(\sigma_{11}-\sigma_{22}\right)^{2}+\left(\sigma_{22}-\sigma_{33}\right)^{2}+\left(\sigma_{33}-\sigma_{11}\right)^{2}\right] \\
& -\sigma_{12} \sigma_{21}-\sigma_{23} \sigma_{32}-\sigma_{31} \sigma_{13}
\end{aligned}
$$

Because of the conservation of angular momentum principle, the stress tensor is symmetric. With this fact in mind, it is tempting to simplify the off-diagonal elements in equation (4), e.g. $\sigma_{12} \sigma_{21}=\sigma_{12}{ }^{2}$. An expression for $J_{2 D}$ will be needed which can be

differenilated with respect to the stress tensor to obtain a proper tersor equation, rence the off-diagonal elements of the stress tensor in equation (4) should not be simplified.

The physical interpretation of the parameter $k$ in equation (1) is that it is the maximum elastic shear stress mulciplied by 2 divided by the square root of 3 . If the state of stress is inside the cylinder, the material is tehaving elastically or rigidly. A state of stress on the surfoce of the cylinder indicates that the material is yielding plastically. Points in Haigh-Westergaard space that lie outside the yield surface are not physically possible. For a perfectly plastic material the parameter $k$ is a constant and the size of the yield surface will not change. For a strain hardening material, $k$ will increase and the yield surface will grow as the material plastically deforms. But $k$ will decrease and the yield surface will become smaller as a strain softening material deforms.

By itself, the yield condition only indicates whether or not the material is undergoing plastic deformation. The plastic deformation itself is governed by the flow rule. The concept of the flow rule states that there is a plastic potential function, $G$, whose gradient with respect to the stress tensor is proportional to the deformation rate tensor. Stated mathematically the flow rule is as follows:

$$
D_{i j}=q \frac{\partial G}{\partial \sigma_{i j}}
$$

where $q$ is a positive scalar that must be determined as part of the solution. The deformation rate teisor, $D_{i j}$, is defined using the velocity vector, $v_{i}$. 


$$
D_{i j}=-\frac{1}{2}\left[\frac{\partial v_{i}}{\partial x_{j}}+\frac{\partial v_{j}}{\partial x_{i}}\right]
$$

Most work in plasticity uses a deformation tensor that is defined with the displacement vector $u_{i}$. A deformation tensor diefined in such a manner requires that the quasi-statical assumption be made. This assumption is often made in soil mechanics because of the very slow velocities present in geotechnical problems. Because this report is investigating the frictional flow of granular materials in situations such as hopper flows where the relocities are much larger, the quasi-statical assumption is not made here.

When transformed to principal soordinates equation (5) becomes:

$$
D_{i}=q \frac{\partial G}{\partial \sigma_{i}}
$$

A common plastic potential function used in both metal and gianular material plasticity is the yield surface. This choice of the yield surface as the plastic potentizl function is known as the associated flow rule. Flow rules which use other plastic potential functions are known as nonassociated flow rules. In order to better visualize the flow rule, it is helpful to superimpose principal deformation rate axec on the corresponding principal stress axes of Haigh-Westergaard space. One can then plot the principal deformation rate vector at the appropriate point on the plastic potential surface. This frocedure for the associated flow rule is shown in Figure 3. Since the rest. of this paper will use the associated flow rule exclusively, equation (7) becomes:

$$
D_{i}=q \frac{\partial F}{\partial \sigma_{1}}
$$

As shown in Figure 3, the ussociated flow rule implies that the deformation rate tensor in principal coordinates is a vector which is normal to the outward surface of the yield condition. With the substitution of the von Mises yield condjtion, equations ( 1 ) and (2), into the associated flow rule, equation (8), the deformation rate tensor can be shown to be equal to:

$$
D_{i}=q\left(\sigma_{1}-<\sigma>\right)
$$

Equation (9) indicates that the principal axes of the deformation rate tensor and the deviatoric stress tensor are aligned. ihis attribute is known as the alignment condition. An important variable in plasticity is the consolidation rate: the crace of the deformation rate tensor. Since the trace of any tensor is 
invariant, one can show that a constitutive relationship consisting of the associated flow rule and the von Mises yield condition implies incompressibi"ity. This follows from equation (9).

$$
\begin{aligned}
D_{i \mathrm{i}} & =D_{1}+D_{2}+D_{3} \\
& =q\left(\sigma_{1}-\langle\sigma\rangle+\sigma_{2}-\langle\sigma\rangle+\sigma_{3}-\langle\sigma\rangle\right) \\
& =q(3\langle\sigma\rangle-3\langle\sigma\rangle)=0
\end{aligned}
$$

Geometrically, in Figure 3 one can see that incompressibility results because any vector normal to the von Mises yield surface possesses no projection on the hydrostatic axis, It should be noted that although ductile metals flow incompressibly, granular materials generaliy do not.

Another important variable in plasticity is $w$, the rate of dissipation of mechanical energy in plastic deformation.

$$
w=\sigma_{i j} D_{i j}=\sigma_{i} D_{i}
$$

Again from Figure 3 , one can see that the angle between the principal stress vector and the principal deformation rate vector is always acute. Therefore $w$, the dot product of the two vectors, is always positive in agreement with experiments and the second Law of Thermodynamics.

The constitutive relationship presented above is well established in metal plasticity. Certain key differences exist between ductile metals and granular materials. Unlike ductile metals, granular materials undergo very small elastic strains and are therefore usually modeled as rigid-plastic materials. Coulomb's law of friction for macroscopic surfaces, equation (12), suggests that the yield condition for granular materials depends linearly on the mean normal stress.

$$
\mathbf{S}=\mathrm{N} \tan \phi
$$

where: $\quad S=$ maximum tangential force

$N=$ normal force

$\tan \phi=$ coefficient of friction

These facts prompted Drucker and Prager (1952) to modify the von Mises yield condition so that the radius of the yield surface is proportional to the hydrostatic stress. This yield surfice is known as the extended von Mises or Drucker-Prager yield condition and is shown in Figure 4. Mathematically the extended von Mises yield condition, $F_{2}=0$, requires the second invariant of the stress tensor to be proportional to the square of the hydrostatic stress. 


$$
F_{2}=-J_{2 D}-k^{2}<\sigma>^{2}=0
$$

According to Schaeffer (1987):

$$
k=\sin \phi
$$

By using the extended von Mises yield condition, equation (13), and the associated flow rule, equation ( 8 ), the following expression for the principal deformation rate vector can be derived.

$$
D_{i}=q\left[\left(\sigma_{i}-\langle\sigma\rangle\right)-\frac{2 k^{2}}{3}\langle\sigma\rangle\right]
$$

As Jackson (1983) discovered, this constitutive model predicts a consistently negative consolidation rate.

$$
\begin{aligned}
D_{i i} & =q\left[\left(\sigma_{1}+\sigma_{2}+\sigma_{3}-3\langle\sigma\rangle\right)-2 k^{2}\langle\sigma\rangle\right] \\
& =-2 q k^{2}\langle\sigma\rangle\langle 0
\end{aligned}
$$

One can visualize this with Figure 4 because all outward normals to the yield surface have negative projections on the hydrostatic axis.

Arother problem with this constitutive relationship is that the energy dissipation for cohesionless granular materials is always zero.

$$
\mathrm{W}=\mathrm{D}_{\mathrm{i}} \sigma_{\mathrm{i}}=2 \mathrm{q}\left(-\mathrm{J}_{2 \mathrm{D}}-\mathrm{k}^{2}<\sigma>^{2}\right)=0
$$

The geometric reason for this lack of energy dissipation, which may be observed in Figure 4 , is that the state of stress vector is a generator of the yield surface, therefore it is orthogonal to all vectors normal to the yield surface.

Schaeffer (1987) used an incompressible constitutive relationship which consisted of the extended von Mises yield condition and a nonassociated flow rule based upon critical state principles. This constitutive relationship guaranteed incompressibility and positive energy dissipation, but his analysis discovered linear ill-posedness. Pitman and Schaeffer (1987) demonstrated that the ill-posedness was caused by incompressibility.

Jenike (1961) formulated a constitutive relationship for the frictional flow of granular materials using a modified form of the Mohr-Coulomb failure envelope as the yield condition and the alignment condition as the flow rule. The Mohr-Coulomb failure envelope generalized for three dimensions using the three stress invariants is: 


$$
F=\langle\sigma\rangle \sin \phi+\left(\cos \alpha-\frac{\sqrt{3}}{3} \sin \phi \sin \alpha\right) \sqrt{-J_{2 D}}=0
$$

where :

$$
\alpha=\frac{1}{3} \sin ^{-1}\left(\frac{9 \operatorname{det}\left(\sigma_{i j}-<\sigma>\delta_{i j}\right)}{2 \sqrt{-3 J_{2 D}}}\right)
$$

In Haigh-Westergaard space, this failure envelope is an irregular hexagonal pyramid with its apex at the origin as shown in Figure 5. The Mohr-coulomb failure envelope was modified by Jenike, Ellsey, and woolery (1960) to permit the yield condition to reflect changes caused by consolidation.

The flow rule used by Jenike is based on the alignment condition and the compressible continuity equation.

$$
D_{i j}=q\left[\sigma_{i j}-\left\langle\sigma>\delta_{i j}\right]+\Gamma \delta_{i j}\right.
$$

where:

$$
\Gamma=\frac{\beta}{3(e+\langle\sigma>)}\left(\frac{\partial<\sigma\rangle}{\partial x_{k}} v_{k}\right)
$$

and $e$ is equal to $11 b / f t^{2}$ and $\beta$ is a material property. Geometrically in Haigh-Westergaard space, this flow rule indicates that the principal deformation rate vector is fixed at the terminal point $E$ of the yield surface. Since the terminal point of the yield surface is a cusp, the consolidation rate is not fixed by the shape of the Mohr-Coulomb yield surface. These facts are illustrated by Figure 5 . The consolidation rate predicted by this flow rule is the trace of equation (20).

$$
D_{i i}=q[3\langle\sigma\rangle-3\langle\sigma\rangle]+3 \Gamma=\frac{\beta}{1+\langle\sigma\rangle}\left(\frac{\partial\langle\sigma\rangle}{\partial x_{k}} v_{k}\right)
$$

Clearly from equation (22) the consolidation rate can be either positive or negative depending upon the stress field. This property is physically realistic.

The rate of energy dissipation predicted by this constitutive relationship can also be derived from equation (20). 


$$
W=\sigma_{i} D_{i}=-2 q J_{2 D}+\frac{\langle\sigma\rangle \beta}{1+\langle\sigma\rangle}\left(\frac{\partial\langle\sigma\rangle}{\partial x_{k}} v_{k}\right)
$$

The predicted rate of energy dissipation should always be positive for any physical system in which frictional forces predominate. At first glance, it appears as if $w$ could be either positive or negative because while the first term of equation (23) is guaranteed to be positive, no such assurance exists for the second term.

In order for $W$ to be positive the absolute value of the second term cannot be larger than the first term if the second term is negative. A comparison of equations (22) and (23) shows that the second term of equation (23) is merely the consolidation rate multiplied by the hydrostatic stress. While the compressibility of a granular material is important dynamically, the actual magnitude of the compressibility is very small. For most granular materials the parameter $\beta$ is much less than 0.1. For an incompressible granular material $\beta$ is equal to zero, and $w$ is always positive. Therefore because of the limited compressibility of granular materials of practical interest, the predicted plastic dissipation rate is assumed to always be positive for this constitutive relationship.

Jenike (1961) used this constitutive relationship to simulate the slow steady state gravity flow of granular materials in converging horizontal and vertical channels. Because he assumes that the flow is both slow and steady, both of the inertia terms in the momentum equation are neglected. This assumption permits the separate calculation of the stress field and the velocity field. The stress field is calculated using the truncated momentum equation and the modified Mohr-coulomb yield condition. The velocity field is then calculated using the continuity equation, the flow rule, and the stress field. Both stress fields and velocity fields are calculated 'sing the method of characteristics. This separation of the problem circumvents the severe analytical difficulties inherent in Jenike's constitutive relationship because the stress tensor is not calculated from the deformation rate tensor. Since one of the objectives of this research is to permit the simulation of more general cases of frictional granular flow, a constitutive relationship without these analytical difficulties is needed.

Recent analytical work by Pitman and Schaeffer (1987) suggests that the steady state two dimensional solutions calculated by Jenike are probably unrealistic because of the presence of illposedness in the evolution equations. These problems are compounded by the use of the modified Mohr-coulomb failure envelope as the yield condition (Pitman, 1989). 
From the above discussion it is clear that a constitutive relationship for the frictional flow of granular materials should be the simplest relationship which can model the phenomenon of dilatation and consolidation while always possessing a positive rate of plastic energy dissipation. Because of its analytical simplicity, the use of the associated flow rule is highly advantageous. Only a constitutive relationship that has a convex yield surface will model the above phenomena, when an associated flow rule is used. Dilatation and consolidation also require that there be a family of nested yield surfaces. Because a granular material with a higher density can resist more stress before deforming than a granular material with a lower density, the yield surfaces for higher densities must enclose those surfaces for lower densities. Each of these yield surfaces has a cross section for which the outward normals have no component along the hydrostatic axis. The granular material at these critical state cross sections will flow incompressibly. In order to accommodate Coulomb's law, these cross secilions form a cone with a half angle equal to the arctangent of the sine of the internal angle of friction. While this cone is identical in shape and size with the extended von Mises cone discussed earlier, the convex yield surface is the yield condition, not the cone.

Gray and stiles (1988) proposed a constitutive relationship which possesses all of these properties. This constitutive relationship consists of the associated flow rule and the following yield condition.

$$
F_{3}=-J_{2 D}+k^{2}\left[(\langle\sigma\rangle-p)^{2}-p^{2}\right]=0
$$

where the parameter $\mathrm{p}$ is the hydrostatic stress when the granular material is flowing in tne critical state, and the parameter $k$ is the sine of the angle of internal friction, $\phi$.

This yield surface is shown in Figure 6 . The length of its major axis in Haigh-Westergaard space is equal to twice the hydrostatic stress in the critical state, $p$. The parameter $p$ is related to the density through the following polytropic equation of critical state.

$$
\mathrm{p}=c \rho^{1 \mathrm{~s}}
$$

Here $c$ and $\beta$ are empirical material constants and $\beta$ is a measure of compressibility numerically similar to the measure of compressibility used by Jenike (1961).

The flow rule can be evaluated by substituting the yield condition, equation (24), into the associated flow rule, equation (8). 


$$
D_{i}=q\left(\sigma_{i}-\langle\sigma\rangle\right)+\frac{2 q k^{2}}{3}(\langle\sigma\rangle-p)
$$

The consolidation rate:

$$
-\frac{\partial v_{i}}{\partial x_{i}}=D_{i i}=2 q k^{2}(\langle\sigma\rangle-p)
$$

is negative for $\langle\sigma\rangle$ less than $p$ (dilatation) and positive for $\langle\sigma\rangle$ greater than $p$ (consolidation), as required by observations and shown in Figure 7. The plastic dissipation rate is always positive as is also illustrated by Figure 7 .

$$
W=\sigma_{i} D_{i}=2 q k^{2}<\sigma>p>0
$$

The transformation of equation (26) from the principal coordinate system to any arbitrary Cartesian coordinate system results in the following equation.

$$
D_{i j}=q\left[\sigma_{i j}+\left(-\langle\sigma\rangle+\frac{2 k^{2}}{3}(\langle\sigma\rangle-p)\right) \delta_{i j}\right]
$$

Together, the yield condition, equation (24), and the associated flow rule, equation (29), provide a constitutive relationship for the three dimensional frictional flow of a rigid-plastic granular material. A necessary condition for the validity of any constitutive relationship is that the equations of continuity and momentum balance and the constitutive relationship define a closed problem. The set of governing equations includes the continuity equation:

$$
\frac{\partial \rho}{\partial t}+v_{j} \frac{\partial \rho}{\partial x_{j}}+\rho \frac{\partial v_{j}}{\partial x_{j}}=0
$$

the momentum equation:

$$
\rho \frac{\partial v_{i}}{\partial t}+\rho v_{j} \frac{\partial v_{i}}{\partial x_{j}}=\rho g_{i}-\frac{\partial \sigma_{i j}}{\partial x_{j}}
$$

the yield condition (24), the associated flow rule (29), the polytropic equation of critical state (25), and the definitions for the deformation rate tensor (6), the second invariant of the deviatoric stress tensor (4), and the hydrostatic stress tensor (3). 
Table 1 demonstrates that this set of governing equations is closed. Given proper boundary conditions, a suitable numerical algorithm should be able to solve this set.

Table 1. Set of Governing Equations.

$\begin{array}{cccc}\text { Equation } & \begin{array}{c}\text { Number of } \\ \text { Equations }\end{array} & \begin{array}{c}\text { Unknowns } \\ \text { Introduced } \\ \rho_{1} v_{i}\end{array} & \begin{array}{c}\text { Number of } \\ \text { Unknowns }\end{array} \\ (30) & 1 & \sigma_{i j} & 4 \\ (31) & 3 & \left.J_{2 D}<\sigma\right\rangle_{,} p & 6 \\ (24) & 1 & q, D_{i j} & 7 \\ (29) & 6 & \text { none } & 0 \\ (25) & 1 & \text { none } & 0 \\ (6) & 6 & \text { none } & 0 \\ (4) & 1 & \text { none } & 0 \\ (3) & 1 & & \end{array}$

While these equations form a closed set, this constitutive relationship is nct free of objections. One limitation is the neglect of elastic deformations. Another is that while the behavior of the granular material in the vicinity of the critical state is probably accurate, the exact shape of the yield surface is probably different from equation (24). While the associated flow rule is desirable mathematically, some investigators have questioned its physical validity. Drescher (1976) experimentally investigated flow rules for granular materials using optically sensitive glass particles immersed in castor oil. The stress and deformation trajectories of these particles were observed while undergoing plane, double simple shear. While these experiments observed misalignment between the principal axes of the deformation rate tensor and the deviatoric stress tensor, the results were not conclusive with regard to the exact nature of the misalignment. Also the applicability of these results to dry granular flow is questionable. Even with these potential problems, this constitutive relationship is more realistic than previous formulations.

Because granular materials are rather difficult to compress, it would seem possible to neglect the small changes in density except where those density variations are dynamically significant. A similar approach is employed when making the Boussinesq approximation for a compressible fluid. But unlike buoyant flows, density variations in frictionally flowing granular materials are dynamically important, not in the buoyancy term, but in the constitutive relationship. The density variations in the flow of a compressible fluid when the Boussinesq approximation is valid can be calculated with the conservation of energy equation. For the frictional flow of granular materials, no such analogous equation 
exists, because variations in temperature do not produce significant variations in density. Therefore, all variations in density for a granular material must be calculated with the complete continuity equation. There can be no "Boussinesq approximation" for granular materials. 


\subsection{CODING THE GRAY-STILES CONSTITUTIVE RELATIONSHIP}

In order to implement the Gray-stiles constitutive relationship given in the preceding section using a hydrodynamic computer program, granular viscosities must be derived and expressed in terms of material constants, the granular density, and the deformation rate tensor. The granular viscosities can easily be derived by comparing the momentum equation for the granular material with the momentum equation for a Newtorian fluid. This derivation was originally presented in Gray and stiles (1989). The stress tensor can be eliminated from the momentum equation, equation (31), using the associated flow rule, equation (29).

$$
\begin{aligned}
\rho \frac{\partial v_{i}}{\partial t}+\rho v_{j} \frac{\partial v_{i}}{\partial x_{j}} & =\rho g_{i}-\frac{\partial p}{\partial x_{i}}+\frac{\partial}{\partial x_{j}}\left(\frac{1}{2 q}\left[\frac{\partial v_{i}}{\partial x_{j}}+\frac{\partial v_{j}}{\partial x_{i}}\right)\right] \\
& +\frac{\partial}{\partial x_{i}}\left[\frac{3-2 k^{2}}{6 q k^{2}}\left[\frac{\partial v_{m}}{\partial x_{m}}\right]\right)
\end{aligned}
$$

The Navier-stokes equation for a compressible, variable property fluid (Batchelor, 1.967) is:

$$
\begin{aligned}
\rho \frac{\partial v_{i}}{\partial t}+\rho v_{j} \frac{\partial v_{i}}{\partial x_{j}}= & \rho g_{i}-\frac{\partial p}{\partial x_{i}}+\frac{\partial}{\partial x_{j}}\left(\mu\left(\frac{\partial v_{i}}{\partial x_{j}}+\frac{\partial v_{j}}{\partial x_{i}}\right)\right) \\
& +\frac{\partial}{\partial x_{i}}\left(\lambda \frac{\partial v_{m}}{\partial x_{m}}\right)
\end{aligned}
$$

where $P=$ fluid pressure

$\mu=$ dynamic viscosity

$\lambda=$ second coefficient of viscosity.

From inspection of equations (32) and (33) the following identifications can be made:

$$
\begin{gathered}
P=p \\
\mu=\frac{1}{2 q} \\
\lambda=\frac{3-2 k^{2}}{6 q k^{2}}
\end{gathered}
$$


The second law of thermodynamic requires that the following inequalities hold (Batchelor, 1967).

$$
\mu \geq 0
$$

$$
\lambda \geq-\frac{2 \mu}{3}
$$

Since $q$ is by definition positive, inequality (37) is always satisfied. Inequality (38) becomes:

$$
\frac{3-2 k^{2}}{6 q k^{2}} \geq \frac{-2}{3}\left(\frac{1}{2 q}\right)
$$

or:

$$
3-2 k^{2} \geq-2 k^{2}
$$

which is obviously satisfied. This is not surprising considering that the plastic dissipation rate is always positive.

The next task is to express the scalar factor $q$ in terms of the invariants of the deformation rate tensor, the material constants, and the density. The associated flow rule, equation (29), can be used to express the second invariant of the deviatoric stress tensor, $J_{2 D}$, in terms of the second invariant of the deviatoric deformation rate tensor, $I_{2 D}$, and the scalar $q$.

$$
\begin{aligned}
J_{2 D}= & -\frac{1}{6}\left[\left(\sigma_{11}-\sigma_{22}\right)^{2}+\left(\sigma_{22}-\sigma_{33}\right)^{2}+\left(\sigma_{33}-\sigma_{11}\right)^{2}\right] \\
& -\sigma_{12} \sigma_{21}-\sigma_{23} \sigma_{32}-\sigma_{31} \sigma_{13} \\
= & -\frac{1}{6 q^{2}}\left[\left(D_{11}-D_{22}\right)^{2}+\left(D_{22}-D_{33}\right)^{2}+\left(D_{33}-D_{11}\right)^{2}\right] \\
& -\frac{1}{q^{2}}\left[D_{12} D_{21}+D_{23} D_{32}+D_{31} D_{13}\right] \\
= & \frac{I_{2 D}}{q^{2}}
\end{aligned}
$$

This relationship can be used to eliminate $J_{2 D}$ in the yield condition, equation (24). 


$$
0=\frac{-I_{2 D}}{q^{2}}+k^{2}(\langle\sigma\rangle-p)^{2}-k^{2} p^{2}
$$

The consolidation rate, equation (27), can then be rearranged and substituted into the second term of equation (41). This can then be solved for $q$ to yield:

$$
q=\frac{1}{2 p k^{2}} \sqrt{D_{i i}^{2}-4 k^{2} I_{2 D}}
$$

This equation can be combined with the equations that relate the granular viscosities with the scalar factor q:

$$
\begin{aligned}
& \mu=\frac{p k^{2}}{\sqrt{D_{i i}^{2}-4 k^{2} I_{2 D}}} \\
& \lambda=\frac{p\left(3-2 k^{2}\right)}{3 \sqrt{D_{i i}^{2}-4 k^{2} I_{2 D}}}
\end{aligned}
$$

When the granular material is rigid, $q=0$, and from equations (35) and (36):

$$
\begin{aligned}
& \lim _{q \rightarrow 0} \mu=\infty \\
& \lim _{q \rightarrow 0} \lambda=\infty
\end{aligned}
$$

Which means that the slower the material deforms, the greiter the resistance to shear, until the material is no longer flowing plastically. In the rigid state, the stresses are governed only by the applied loading. There is not even a smooth transition between the plastic and rigid regimes because:

$$
\lim _{q \rightarrow 0}\left(\mu D_{i j}\right)=\left(\sigma_{i j}-<\sigma>\delta_{i j}\right) / 2
$$

This lack of a smooth transition between rigid and deforming states is not observed in Newtonian fluids and is responsible for the numerical difficulty in modeling the onset of plastic flow in a rigid-plastic material. 


\subsection{Evaluation of the Material Properties}

In order to use the constitutive relationship proposed by Gray and Stiles (1988), numerical values must be assigned to the empirical constants in the equation of critical state (25). These values are assigned using the results of direct shear and virgin compressibility tests reported by Royal (1986) with the yield condition used by the proposed constitutive relationship. This work was originally presenter in Gray and stiles (1990).

A schematic of a shear cell from Cernica (1982) is shown in Figure 8. With the results from the direct shear test one can plot the shear stress versus the normal stress in the critical state. For most granular materials the plot is a straight line. The $y$ intercept of this line is called the cohesion of the material and the slope is $\tan \phi$ where $\phi$ is the angle of internal friction. When the granular material yields, the relationship between shear stress and normal stress is given by the plot which is referred to as the failure envelope. A corresponding Mohr circle can be drawn for any state of stress on the failure envelope. This Mohr circle can be used to calculate the major and minor principal stresses. Because the shear cell resists deformation normal to the applied normal and shear loads and no load is applied in that direction, the direct shear test is a plane strain experiment. Therefore, the principal stress normal to both the major and minor principal stresses is equal to the average of the major and minor principal stresses. An example of a plot of the failure envelope and a corresponding Mohr circle is shown in Figure 9.

The yield condition proposed by Gray and stiles (1988) is:

$$
F_{3}=-J_{2 D}+k^{2}\left((\langle\sigma\rangle-p)^{2}-p^{2}\right)=0
$$

Because the granular material yields along the failure envelope, equation (24) is valid at any point along the failure envelope. Rearranging equation (24) and solving for $p$ yields:

$$
p=\frac{1}{2}\left(\langle\sigma\rangle-\frac{J_{2 D}}{\langle\sigma\rangle k^{2}}\right)
$$

From the geometry of the failure envelope and the corresponding Mohr circle, the major and minor stresses are:

$$
\sigma_{1}=\sigma\left(1+\tan ^{2} \phi\right)(1+\sin \phi)
$$




$$
\sigma_{3}=\sigma\left(1+\tan ^{2} \phi\right)(1-\sin \phi)
$$

where: $\sigma=$ applied normal load. Because of the plane strain condition:

$$
\begin{gathered}
\sigma_{2}=\left(\sigma_{1}+\sigma_{3}\right) / 2 \\
\langle\sigma\rangle=\left(\sigma_{1}+\sigma_{3}\right) / 2
\end{gathered}
$$

Equations (49) through (52) can be used to evaluate the second invariant of the deviatoric part of the stress tensor.

$$
-J_{2 D}=\frac{-1}{4}\left(\sigma_{1}-\sigma_{3}\right)^{2}=-\left[\sigma\left(1+\tan ^{2} \phi\right) \sin \phi\right]^{2}
$$

Equations (49) and (50) can be used with equations (51) and (52) to calculate the hydrostatic stress.

$$
<\sigma>=\sigma\left(1+\tan ^{2} \phi\right)
$$

Equations (53) and (54) can be used to evaluate the hydrostatic stress present when the granular material is in the critical state.

$$
p=\sigma\left(1+\tan ^{2} \phi\right)
$$

Because there is shear acting in the same plane as the normal stress applied to the shear cell, the applied normal stress, $\sigma$, is not equal to the major principal stress, $\sigma_{1}$. The density of the granular material is a function of the major principal stress only (Royal, 1986), so equation (55) must be expressed in terms of $\sigma_{1}$.

$$
p=\frac{\sigma_{1}}{1+\sin \phi}
$$

Royal (1986) also made tests of the virgin compressibility of several granular materials. A schematic diagram of the standard compressibility tester is shown in Figure 10 (Royal, 1986). With the virgin compressibility test, a common assumption is that no shear stress is present in the same plane as the applied normal stress, therefore the major principal stress, $\sigma_{1}$, is equal to the applied normal stress. Modern techniques of granular material testing almost eliminate the friction between the sample and the testing apparatus and ensure that this assumption is reasonable. The results of the compressibility tests were used to fit a compressibility equation of the form: 


$$
\rho=\max \left[\rho_{0}\left(\frac{\sigma_{1}}{\sigma_{0}}\right)^{\beta}, \rho_{\mathrm{m}}\right)
$$

where $\rho_{\mathrm{m}}$ is the minimum density of the granular material, and $\sigma_{0}$ and $\rho_{0}$ are the initial normal stress applied to and the initial density of the granular material during the compressibility test. The form of equation (57) reflects the fact that the density during virgin compression is a function only of the major principdl stress when the density is above some minimum value. Equation (56) can be solved for $\sigma_{1}$ and used in equation (57), resulting in:

$$
\rho=\max \left[\rho_{0}\left(\frac{\mathrm{p}(1+\sin \phi)}{\sigma_{0}}\right)^{\beta}, \rho_{\mathrm{m}}\right]
$$

Equation (58) can be arranged to make an equation with a form similar to equation (25).

$$
\rho=\max \left(a p^{\beta}, \rho_{m}\right)
$$

whe? I:

$$
a=\rho_{0}\left(\frac{1+\sin \phi}{\sigma_{0}}\right)^{\beta}=c^{-\beta}
$$

Equation (60) was used to fit $a$ and $\beta$ by regression for six cohesionless granular materials with results shown in Table 2 . Because equation (57) is valid only over a range of densities, Table 2 also contains the range of densities for which equation (57) is valid. Figures 11 through 16 are plots of the critical state pressure, $p$, versus the density, $\rho$, on logarithinic axes for each granular material. The solid line represents the equation of critical state and the dashed lines represent the probable amount of error in the estimation of the parameters a and $\beta$. The probable amount of error for the parameters is also given in Table 2 for each granular material. The numerical values assigned to a and $\beta$ are reasonable, but the experimental results from other investigators will be needed to confirm these values. 
Table 2. Compressibility Parameters for Several Coals.

\begin{tabular}{|c|c|c|c|c|}
\hline No. & Description & Particle siz & $\mathrm{ze}$ & Moisture Conte \\
\hline 1 & Illinois $5 / 6$ & -8 Mesh & & $3.15 \%$ \\
\hline 2 & Pitt. Seam Bit. & $-1 / 4$ Inch & & $17.20 \%$ \\
\hline 3 & Indianhead Lig. & $-1 / 4$ Inch +20 & Mesh & $35.80 \%$ \\
\hline 4 & Indianhead Lig. & $-1 / 4$ Inch +20 & Mesh & 40.758 \\
\hline 5 & Wyodak sub-Bit. & $-1 / 4$ Inch & & 30.408 \\
\hline$\epsilon$ & Wyodak sub-Bit. & $-1 / 4$ Inch & & 30.708 \\
\hline No. & $\mathbf{a}$ & $\beta$ & Min. $\rho$ & $\operatorname{Max} . \rho$ \\
\hline 1 & $0.62180 \pm 0.00020$ & $0.0095 \pm 0.0002$ & 0.74 & 0.78 \\
\hline 2 & $0.40260 \pm 0.00040$ & $0.0278 \pm 0.0005$ & 0.67 & 0.78 \\
\hline 3 & $0.36050 \pm 0.00040$ & $0.0231 \pm 0.0010$ & 0.55 & 0.62 \\
\hline 4 & $0.18069 \pm 0.00002$ & $0.0550 \pm 0.0001$ & 0.50 & 0.66 \\
\hline 5 & $0.50140 \pm 0.00030$ & $0.0171 \pm 0.0005$ & 0.68 & 0.76 \\
\hline 6 & $0.51860 \pm 0.00030$ & $0.0155 \pm 0.0005$ & 0.64 & 0.68 \\
\hline
\end{tabular}

Note: Units of density are $\mathrm{g} / \mathrm{cm}^{3}$.

3.2 Results Using the Gray-stiles Consititutive Relationship

The formulas for equavilent granular pressure, dynamic viscosity, and bulk viscosity, equations (34), (43), (44), were used to implement the frictional granular constitutive relationship in TEACH. Density changes were modeled with the equation of state, (25). These modifications were implemented in subroutine PROPS.

In addition to the numerical difficulties inherent in the von Mises constitutive relationship which are described below, the frictional constitutive relationship possesses additional numerical problems when implemented in two dimensions. As was discussed by schaeffer, Shearer, and Pitman (1988), the governing equations for two 
dimensional flow change from elliptic to hyperbolic as the material approaches the critical state from the consolidation side of the yield surface. When the critjcal state is reached, the equations become ill-posed. Pitman and Schaeffer (1988) conjectured that the formation of shear bands in granular materials were caused by this ill-posedness. All attempts to simulate the frictional flow of a Gray-Stiles granular material using the computer program TEACH were completely unsuccessful. 


\section{0 CODING THE VON MISES CONSTITUTIVE RELATIONSHIP}

As was mentioned earlier, the proposed governing equations for the frictional flow of granular materials are superficially similar to the equations governing the flow of a compressible fluid. Because of the complexity of this set of equations, it is desirable to test any proposed numerical algorithm with a simple constitutive relationship that retains the basic qualitative properties of the frictional granular relationship. One of the basic properties of the frictional granular relationship is the property of rateindependency. This indicates that the constitutive relationship when expressed as a relationship between the stress tensor and the deformation rate tensor, does not contain any terms with a time derivative of the stress tensor.

Because of its application in geomechanics, the extended von Mises constitutive relation proposed by Drucker and Prager appears to be a good choice. Unfortunately, the presence of a negative definite consolidation rate prevents the incorporation of the extended von Mises constitutive relationship in a hydrodynamic code without major modifications to the continuity equation. Since the goal is to develop a numerical algorithm to solve the set of governing equations proposed by Gray and stiles (1988), the use of the extended von Mises constitutive relationship would be selfdefeating.

The von Mises constitutive relationship, because it implies incompressibility, does not suffer from the aforementioned liability, and the basic features of the frictional constitutive relationship, such as rate-independency, are retained. Because the computer program TEACH was originally designed to model subsonic flows with density variations, the incompressibility inherent in the von Mises constitutive relationship causes no numerical problems. The von Mises constitutive relationship consists of the yield condition,

$$
F_{1}=-J_{2 D}-k^{2}=0
$$

and the associated flow rule.

$$
D_{i j}=q \frac{\partial F}{\partial \sigma_{i j}}
$$

Evaluating the associated flow rule and solving for the stress tensor yields:

$$
\sigma_{i j}=\langle\sigma\rangle \delta_{i j}+\frac{D_{i j}}{q}
$$

An equation for the plastic scalar, $q$, can be formulated by substituting equation (62) into (1). 


$$
q=\frac{\sqrt{-I_{2 D}}}{k}
$$

Substituting equation (63) into equation (62) expresses the stress tensor in terms of the mean normal stress and the deformation rate tensor.

$$
\sigma_{i j}=\langle\sigma\rangle \delta_{i j}+\frac{k}{\sqrt{-I_{2 D}}} D_{i j}
$$

The stress tensor, using compressive notation, for an incompressible Newtonian fluid is (Batchelor, 1967):

$$
\sigma_{i j}=p \delta_{i j}+2 \mu D_{i j}
$$

A comparison of equations (64) and (65) leads to the following expressions for pressure and granular dynamic viscosity.

$$
\begin{gathered}
P=\langle\sigma\rangle \\
\mu=\frac{k}{2 \sqrt{-I_{2 D}}}
\end{gathered}
$$

The expression for pressure, equation (66), indicates that a numerical algorithm for modeling the flow of a von Mises material can calculate the pressure in the same manner as for an incompressible fluid. Because of the form of equation (67), the behavior of the von Mises material as the deformation becomes slower is identical to the behavior of the frictional granular material as described by inequalities (45), (46), and (47).

\subsection{Results Using the Von Mises Constitutive Relationship}

In its original form, the computer program TEACH models the subsonic turbulent steady-state flow of a fluid in a two dimensional or axisymmetric duct. The objective of these simulations is to model the two dimensional plane-strain Poiseuille flow of a von Mises material. Clearly, this required substantial modification to TEACH. The equations being solved in these simulations include continuity,

$$
\frac{\partial u}{\partial x}+\frac{\partial v}{\partial y}=0
$$

x-momentum, 


$$
\begin{aligned}
\rho u \frac{\partial u}{\partial x}+\rho v \frac{\partial u}{\partial y} & =-\frac{\partial p}{\partial x}+\frac{\partial}{\partial x}\left(\frac{k}{\sqrt{-I_{2 D}}} \frac{\partial u}{\partial x}\right) \\
& +\frac{\partial}{\partial y}\left(\frac{k}{2 \sqrt{-I_{2 D}}}\left(\frac{\partial u}{\partial y}+\frac{\partial v}{\partial x}\right)\right)
\end{aligned}
$$

$y$-momentum,

$$
\begin{aligned}
\rho u \frac{\partial v}{\partial x}+\rho v \frac{\partial v}{\partial y} & =-\frac{\partial p}{\partial y}+\frac{\partial}{\partial y}\left(\frac{k}{\sqrt{-I_{2 D}}} \frac{\partial v}{\partial y}\right) \\
& +\frac{\partial}{\partial x}\left(\frac{k}{2 \sqrt{-I_{2 D}}}\left(\frac{\partial u}{\partial y}+\frac{\partial v}{\partial x}\right)\right)
\end{aligned}
$$

and the second invariant of the deformation rate tensor.

$$
\begin{aligned}
I_{2 D}= & \left.-\frac{1}{6}\left[\frac{\partial u}{\partial x}+\frac{\partial v}{\partial y}\right)^{2}+\left(\frac{\partial u}{\partial x}\right)^{2}+\left(\frac{\partial v}{\partial y}\right)^{2}\right] \\
& -\frac{1}{4}\left(\frac{\partial u}{\partial y}+\frac{\partial v}{\partial x}\right)^{2}
\end{aligned}
$$

The first change to TEACH involved setting the geometry variable INDCOS equal to 1 so that two dimensional Cartesian coordinates would be employed. Because symmetry boundary conditions can sometimes cause numerical problems, it was decided to replace the symmetry condition with a solid boundary. The no-slip boundary condition was imposed at both the upper and lower solid boundaries of the flow. Originally TEACH had a boundary condition at the inlet which allowed the user to specify the velocity distribut:ion on that line, and a free flow condition at the outlet which maintained overall continuity for the entire computational domain. These two boundary conditions were retained in this version.

Implementing the von Mises constitutive relationship involved using equation (67) to calculate the dynamic viscosity. Because equation (67) is an expression for dynamic viscosity in terms of the velocity gradients, this version of TEACH updates the dynamic viscosity each iteration using the velocity field calculated by the previous iteration. This is performed in subroutine PROPS. BY inspection, equation (67) clearly indicates that the dynamic viscosity is inversely proportional to the deformation rate. When the deformation rate is very small, the dynamic viscosity approaches infinity. This problem is alleviated by specifying a 
maximum value for the dynamic viscosity; these simulations restricted it to a maximum of $5.0 \times 10^{5} \mathrm{~N}-\mathrm{s} / \mathrm{m}^{2}$.

After several simulations, it became clear that the numerical behavior of a von Mises material was quite different from an incompressible Newtonian fluid, and it was difficult to decide when a simulation had converged. A minimum standard for convergence for any iterative numerical algorithm is that the variables being calculated do not change very much between successive iterations. This was selected as the primary convergence criterion for these simulations. The relative change in the calculated values of velocity, pressure, and dynamic viscosity are calculated and printed at the end of each juteration. The simulations were said to have converged when the relative change in the primary variables (pressure, $u-$, and v-velocity) were less than 0.1 percent. While the relative change in the dynamic viscosity was monitored, it was not employed to determine convergence since its value only reflects the calculated velocity field.

The version of TEACH used to simulate the Poiseuille flow of a von Mises material is listed in Appendix 3, where all modifications to TEACH are identified by the use of lower case letters. While the use of lower case letters is not allowed in ANSI FORTRAN-77, most compilers permit their use, including the VAX FORTRAN compiler employed for these simulations.

The simulation presented in this report is of the Poiseuille flow of a von Mises material between two plates $0.02 \mathrm{~m}$ apart. The length of the computationa' domain is $2 \mathrm{~m}$. A plot of the computational grid is shown in Figure 17. For clarity's sake the vertical scale in all of the plots presented here is 100 times larger than the horizontal scale. In Figure 17 the solid boundary celis at the top and bottom of the computational domain are represented by cross hatching. The inflow boundary cells are shown with left hatching, and the outflow cells are shown with right hatching.

A parabolic velocity distribution was specified at the entrance to the flow field and was used as the initial condition. This velocity distribution was specified because the shear stress is distributed along the width of the flow field without any discontinuities in the velocity gradient. The initial centerline velocity of the flow was $0.1 \mathrm{~m} / \mathrm{s}$. The parameter $\mathrm{k}$ used in equation (67) for the dynamic viscosity was arbitrarily set to $1 \mathrm{~Pa}$ which corresponds to a yield strength of $0.866 \mathrm{~Pa}$. Body forces were neglected in these simulations.

strong underrelaxation of the velocities was required for convergence, and the underrelaxation factors were set to 0.4 . The pressure calculations were underrelaxed with a value of 0.9 . Numerical experiments with larger values resulted in monotonic 
divergence. While it was possible to underrelax the viscosity calculations, it was felt that to do so would create dynamic problems due to its relationship with the velocity field.

Figure 18 is a vector plot of the velocity field after 500 iterations. As expected, the flow is primarily unidirectional. on the left side of Figure 18 an almost parabolic velocity distribution is shown, and as flow progresses it becomes more uniform in the center of the field. At the outlet, on the right side, the von Mises material is flowing almost as a rigid body in the middle third of the domain with the majority of the shearing in a layer near each wall. This lack of significant diffusion in the middle of the flow occurs because the dissipative mechanism is that of friction between the particles rather than diffusion as for a Newtonian fluid. Similar behavior has been observed in flowing granular materials.

Figure 19 is a plot of the relative pressure contours after 500 iterations. Overall the relative pressure is, paradoxically, increasing in the direction of flow. Near the inlet there is a sharp drop, then the pressure remains constant over the second and third tenth's of the flow field. This behavior can probably be attributed to entrance effects because this relatively constant region is at approximately the same location as where the shear layer forms. Over the remaining seven tenth's of the flow field the relative pressure is monotonically increasing. In the middle of this region, the pressure contours are curved, probably due to the increased velocity in this region. This seemingly paradoxical behavior can be explained by examining what happens to the $x-$ momentum equation. In the middle of the flow near the outlet the following conditions hold true.

$$
\begin{gathered}
\frac{\partial u}{\partial y} \rightarrow 0 \\
\frac{\partial u}{\partial x} \rightarrow 0 \\
v \rightarrow 0
\end{gathered}
$$

These conditions result in the following changes to the $x$-momentum equation.

$$
\begin{aligned}
& \rho u \frac{\partial u}{\partial x} \rightarrow 0 \\
& \rho v \frac{\partial u}{\partial y} \rightarrow 0
\end{aligned}
$$




$$
I_{2 D} \rightarrow 0
$$

This result causes the following dispersion terms in the momentum equation to become indeterminate.

$$
\begin{gathered}
\frac{\partial}{\partial y}\left[\frac{k}{2 \sqrt{-I_{2 D}}}\left(\frac{\partial u}{\partial y}+\frac{\partial v}{\partial x}\right)\right) \\
\frac{\partial}{\partial x}\left(\frac{k}{\sqrt{-I_{2 D}}} \frac{\partial u}{\partial x}\right)
\end{gathered}
$$

By elimination, the remaining term of the $x$-momentum equation, the pressure gradient, has to be indeterminate. This indicates that whatever pressure variation is calculated under these conditions by TEACH has nothing to do with the flow field. similarly, the pressure gradient in the $y$-direction is also indeterminate.

Figure 20 is a vector plot of the velocities after 1000 iterations. overall the velocity field has not changed much in the previous 500 iterations. The flow is slightly more uniform in the center of the domain, and the shear layers form slightly farther from the inlet. Figure 21 is a contour plot of the relative pressure after 1000 iterations. The qualitative behavior of the pressure field has not changed much with respect to the location of the formation of the shear layers.

Figure 22 is as vector plot of the velocities after 10,000 iterations. At this point, the shear layers have become thinner than the width of the computational cells closest to the wall. The velocity field is completely uniform and unidirectional throughout the entire computational domain. The relative change in the velocities at this point is less than 0.001 percent. The magnitude of the velocity is quite small, less than $68 \%$ of the initial centerline velocity. It would appear that the effect of decoupling the pressure field from the momentum equation has spread to the entire flow field.

Figure 23 is a contour plot of the relative pressure after 10,000 iterations. This plot confirms that the momentum equation has been completely decoupled from the velocity distribution. A positive pressure difference of $2000 \mathrm{~Pa}$ is shown between the inlet and outlet. Although the relative change in pressure after this iteration is greater than 10 percent, the simulation appears to have reached a steady-state. To reach this point the calculations required approximately 63 minutes of CPU time on the Morgantown Energy Technology Center's VAX 8650.

The results of this simulation appear to indicate that as the flow of the von Mises material becomes fully developed (e.g. as the 
shear layers develop) the velocity distribution becomes disengaged from the momentum equation. While $\mathrm{TEACH}$ is a steady-state code, it is possible to infer the transient behavior by viewing the simulation at various locations along the direction of flow. When the material is shearing continuously, like at the inlet, the velocity distribution and the momentum equation are properly coupled. Because the von Mises constitutive relationship is shear thinning, the resistance to deformation is inversely proportional to the deformation rate. This results in regions of almost rigid motion appearing near the outlet, and the influence of the crossstream derivative of the velocity on the streamwise momentum equation becomes greatly diminished. After an almost infinite time, the shear layers become smaller than the width of the computational cells next to the walls and the material flows almost as a rigid body. This behavior is a consequence of inequalities $(45),(46)$, and (47).

Inspection of the velocity field calculated by this simulation, indicates that proper kinetic energy dissipation is occurring when the material is continuously shearing. Where the material is flowing as a rigid body, the dissipation rate approaches zero. This behavior is not surprising considering the decoupling of the velocity distribution from the momentum equation that occurs when the material is moving as a rigid body.

Because of the flat velocity profile and the decoupling of the velocity field from the momentum equation in the poiseuille flow simulations, it was decided to simulate Poiseuille-couette flow to determine if the velocity boundary conditions were being enforced properly. These simulations were conducted by first simulating Newtonian flow and then switching to the von Mises constitutive relationship. This change in constitutive relation would be gradual because the under-relaxation factor for the granular dynamic viscosity was set to 0.1 . The boundary conditions for these simulations were the same except for the top solid boundary, which was moved in the positive $x$ direction at a constant velocity. The computational grid employed by these simulations used a finer mesh near the top and bottom solid boundaries and is shown by the plots of the velocity profiles. A cell width of 0.25 m was used over most of the grid in the horizontal direction with a smaller width of $0.2 \mathrm{~m}$ used near the inflow and outflow boundaries. As in the Poiseuille simulations, the total number of cells in the $y$ direction was 26 ; to conserve computational resources, the total number of cells in the $x$ direction was reduced to 23 . The size of the flow field was $0.1 \mathrm{~m}$ by $5.0 \mathrm{~m}$.

The value selected for the viscosity for the Newtonian part of the simulations was based upon the von Mises equation for the dynamic viscosity and the assumption that the cross stream derivative of the horizorital velocity would be dominant. This value was calculated using the following FORTRAN formula. 


$$
M U=K * R L A R G E / U T O P=5900 N-s / m \star \star 2
$$

where:

$$
\begin{aligned}
& \mathrm{K}=5.9 \mathrm{E}+04 \text { Pascals } \\
& \text { RLARGE }=\text { width of flow field }=0.1 \mathrm{~m} \\
& \text { UTOP = velocity of sliding top plate } 1.0 \mathrm{~m} / \mathrm{s}
\end{aligned}
$$

Figure 24 is a plot of the velocity profiles for the Newtonian simulation at several locations along the length of the computational domain. The left-most velocity profile is the velocity profile specified at the inflow area. The right-most profile is the condition specified at the outflow to ensure satisfaction of continuity for the ccmputational domain. Figure 25 is a plot of the pressure at the center of the flow field versus horizontal distance for the Newtonian simulation. This plot shows the expected linear drop in pressure in the direction of flow. As expected, there is no vertical pressure gradient.

It was initially believed that the simulations of Poiseuille flow discussed earlier were in error because of the restriction given to the maximum size of the granular dynamic viscosity. As the Poiseuille simulations reached steady state, the velocity profiles became flat and the granular dynamic viscosity over most of the flow field became equal to that maximum value. Some modification to the von Mises relation was required to prevent floating point overflow errors from occurring in the center of the flow field where the velocity gradients were small. Because the kinetic energy dissipation approaches zero as the velocity profile becomes flat, it was decided to set the granular dynamic viscosity equal to zero when the calculated viscosity would have been larger than the initial viscosity. Figure 26 is a plot of the velocity profiles for this simulation 1000 iterations after the switch from the Newtonian relation. This simulation is clearly unsuccessful because of the presence of a velocity discontinuity in the upper half of the two right-most velocity profiles. Figure 27 is a plot of the pressure at the center of the flow field versus horizontal distance. This plot shows a general drop in pressure between the inflow and outflow boundaries, but there is no apparent reason for the change of pressure gradient in the middle of the flow field.

After the failure of the previous simulation, it was decided to go back to the previous method of restricting the maximum value of the granular dynamic viscosity. This simulation specified the maximum viscosity to be equal to the initial viscosity. Figure 28 is a plot of the velocity profiles for this simulation 1000 iterations after the switch from the Newtonian relation. At this iteration level the velocity field satisfies the boundary conditions at the top and bottom (no-slip), but in the middle of the field the material is flowing as a rigid body similar to the von Mises 
simulations discussed above. At this point, it appears that the simulation has reached a steady state. It appears that the shearing boundary conditions prevent the shear layers from thinning out. While the decoupling of the velocity field from the momentum equation, observed in the Poiseuille simulations, probably does occur in the middle of the flow where the profile is flat, the midline pressure plot, Figure 29, appears to be reasonable. Inspection of the printed output indicates that no vertical pressure gradient is present.

With the reasonably successful simulation of Couette-Poiseuille flow described above, the authors were curious as to the effect of adding kinetic stress terms to the von Mises constitutive relation. The addition of kinetic stress terms to the frictional granular stress tensor is often employed by investigators to regularize the system of governing equations (Pitman, 1988). With this in mind, the formulas for the kinetic pressure and dynamic viscosity used in the original version of the program MFIX were added to the pressure and granular dynamic viscosity calculated by program TEACH. Because the von Mises constitutive relationship 1 mplies incompressibility, the kinetic effects caused by volumetric deformations were ignored.

Figure 30 is a plot of the velocity profiles for this simulation 1000 iterations after the switch from the Newtonian relation, and Figure 31 is a plot of the pressure at the center of the flow field verslis horizontal distance. Neither Figure 30 nor Figure 31 shows any change from the results of the non-kinetic von Mises simulation described earlier. It would appear that the von Mises constitutive relation predominates throughout the flow field. 


\subsection{CONCLUSIONS}

The goals of validating a constitutive law for the frictional flow of granular materials and incorporating this law into a general granular flow code have not been achieved. Using a phenomenological approach based on the theory of plasticity, a new constitutive law was proposed. This Gray-stiles law consists of (1) a yield surface family which allows for consolidation, dilatation, and critical state flow and (2) the associated flow rule which guarantees alignment of the principal axes of stress and deformation rate. Appendix 1 descripes generalizations which permit cohesion and non-coulombic behavior. While further refinements are certainly possible, the authors have no doubt that this formulation is the most realistic phenomenological law yet proposed for frictional flow.

on the other hand, extensive attempts to use the Gray-stiles law as a basis for computations were completely unsuccessful. In order to simplify th.e numerical problems, computations were carried out using the vo.r Mises constitutive law. These experiments provided some insight into the difficulties experienced with the Gray-stiles formulation.

The TEACH simulation of Poiseuille flow with the von Mises constitutive relationship shows a decoupling of the pressure field from the momentum equation as the simulation approaches a steadystate with a uniform flat velocity profile. As was shown earlier, this decoupling was the result of the inertia terms of the momentum equation vanishing and the dispersion terms becoming indeterminate.

The flat velocity profile of the steady-state solution indicates that very little diffusion of momentum occurred in the flow field. Under certain conditions granular materials will flow as rigid blocks with the vast majority of the momentum transfer occurring in the shear bends separating the rigid blocks. The mechanism for momentum transfer across these shear bends is probably collisional (Pitman and Schaeffer, 1988). Collisional effects that are caused by the particles behaving like gas molecules were not modeled in most of these simulations. The lack of significant momentum diffusion in frictional flow is a result of the type of dissipative mechanism present in granular materials. Newtonian fluids dissipate kinetic energy by diffusing momentum to the surrounding fluid. As a frictional granular material flows, the kinetic energy is dissipated by the particles rolling and sliding over one another. This results in the kinetic energy being dissipated in the form of thermal energy via friction. Because of the large specific heat values for solids, this dissipation results in minuscule changes in temperature.

With the experience gained from this research, it is possible to identify some required characteristics of a numerical algorithm for 
modeling the frictional flow of a granular material. These attributes include:

1. The ability to model three dimensional flow to avoid the ill-posedness discovered by schaeffer and Pitman (1988).

2. The ability to adjust to changes in the type of the steady-state equations (Pitman, 1989). This probably should include the ability to capture shocks.

3. The ability to model flows with a large and highly variable viscosity. The need for this attribute was shown by the von Mises simulations.

No numerical algorithm known to the authors, possesses all of these attributes. This absence of such an algorithm in the literature and the failure to develop one explains the limited success of this research.

While the simulations of the flow of a granular material using the Gray-stiles constitutive relationship were not successful, the von Mises simulations do show some features that are qualitatively similar to observed phenomena in frictionally flowing granular materials. This research has also helped clarify the problems that remain to be solved in order to model the frictional flow of a granular material.

\subsection{ACKNOWLEDGEMENT}

The authors are most grateful to Dr. Edward J. Boyle of METC for his assistance and encouragement. 


\subsection{REFERENCES}

Batchelor, G.K., 1967. An Introduction to Fluid Dynamics, Cambridge University Press, London.

Baxter, G.W., R.P. Behringer, T. Faggert, and G.A. Johnson, 1989. "Pattern Formation in Flowing Sand", Physical Review Letters, 62, 2825-2828.

Bland, D.R., 1957. "The Associated Flow Rule of Plasticity," Journal of the Mechanics and Physics of Solids, $\underline{6}, 71-78$.

Boyle, E.J., M. Massoudi, and T.J. O'Brien, 1988. "A Kinetic Theory Derivation of the stress Tensor for Granular Material That Includes Normal Stress Effects", Engineering Mechanics7th Conference, American Society of Civil Engineers, New York, 22 .

Brown, R.I., and J.C. Richards, 1970. Principles of Powder Mecharics, Pergamon Press, London.

Cernica, J.N., 1982. Geotechnical Engineering, CBS College Publishing, New York.

Cundall, P.A., and O.D.L. Strack, 1979. "A discrete numerical model for granular assemblies", Géotechnique, 29, 47-65.

Drescher, A., 1976. "An experimental investigation of flow rules for granular materials using optically sensitive glass particles", Géotechnique, 26, 591-601.

Drucker, D.C., 1950. "Some Implications of Work-Hardening and Ideal Plasticity," Quarterly of Applied Mathematics, I, 411-418.

Drucker, D.C., 1951. "A More Fundamental Approach to Plastic Stress-strain Relations," Proceedings of the First U.S. National Congress on Applied Mechanics, 487-491.

Drucker, D.C., and W. Prager, 1952. "Soil Mechanics and Plastic Analysis or Limit Design", Quarterly Journal of Applied Mathematics, 10, 157-165.

Gray, D.D., and J.M. Stiles, 1988. "On the constitutive Relation For Frictional Flow of Granular Materials", Topical Report DOE/MC/21353-2584 (DE88001089), U.S. Department of Energy. 
Gray, D.D., and J.M. Stiles, 1989. "Theoretical and Numerical Studies of Constitutive Relations for Frictional Granular Flow", Topical Report, U.S. Department of Energy, preprint.

Gray, D.D., and J.M. Stiles, 1990. "Theoretical and Numerical Studies of Constitutive Relations for Frictional Granular Flow", Annual Report January - December 1989 DOE/MC/24207-2852 (DE90009662), U.S. Department of Energy.

Jackson, R., 1983. "Some Mathematical and Physical Aspects of Continuum Models for the Motion of Granular Materials", Theory of Dispersed Multiphase Flow, R.E. Meyer ed., Academic Press, New York, 291-337.

Jenike, A.W., P.J. Elsey, and R.H. Woolley, 1960. "Flow Properties of Bulk Solids", Proceedings ASTM, $\underline{60}$, 11681181.

Jenike, A.W., 1961. "Gravity Flow of Bulk Solids", Utah Engineering Experiment Station, Bulletin 108, University of Utah.

Jenkins, J.T., and S.B. Savage, 1983. "A Theory for the Rapid Flow of Identical, Smooth, Nearly Elastic spherical Particles", Journal of Fluid Mechanics, 130, 187-202.

Mehrabadi, M.M., 1986. "Modeling of Rate-Independent Flow of Granular Materials", Proceedings of the Tenth U.S. National Congress of Applied Mechanics, ASME.

Patankar, S.V., 1980. Numerical Heat Transfer and Fluid Flow, Hemisphere, Washington, D.C.

Pitman, E.B., and D.G. Schaeffer, 1987. "stability of Time Dependent Compressible Granular Flow in Two Dimensions", Communications on Pure and Applied Mathematics, 40, 421447 .

Pitman, E.B., and D.G. Schaeffer, 1988. "Instability and IllPosedness in Granular Flow", Proceedings AMS Workshop on Riemann Problems, preprint.

Pitman, E.B., 1989. Personal Communication.

Royal, T.A., 1986. "Analysis of Pressurization of Lock Hoppers and Typical Measured Flow Properties of Coal", Phase II, AC21-80MC14101, U.S. Department of Energy.

Schaeffer, D.G., 1987. "Instability in the Evolution Equations Describing Granular Flow", Journal of Differential Equations, 66, 19-50. 
Schaeffer, D.G., and E.B. Pitman, 1988. "Ill-Posedness in ThreeDimensional Plastic Flow", Communications on Pure and Applied Mathematics, $\underline{41}$, 879-890.

Schaeffer, D.G., M. Shearer, and E.B. Pitman, 1988. "Instability in Critical state Theories of Granular Flow", preprint.

Schofield, A., and P. Wroth, 1968. Critical state soil Mechanics, McGraw-Hill, New York.

Syamlal, M., 1985. Multiphase Hydrodynamics of Gas-Solids Fl. OW, Unpublished Doctoral Dissertation, Ilinois Institute of Technology.

Syamlal, M., 1987. "NIMPF: A Computer Code for Nonisothermal Multiphase Fluidization", Unpublished EG\&G Report.

Syamlal, M., 1988. Personal Communication.

Walton, O.R., R.L. Braun, and D.M. Cervelli, 1986. "Flow of Granular Solids: 3-Dimensional Discrete-Particle Computer Modeling of Uniform Shear", U.S. DOE Office of Fossil Energy, PETC/METC Direct Utilization \& AR\&TD Contractor Review Meeting, preprint. 
7.0 Figures 


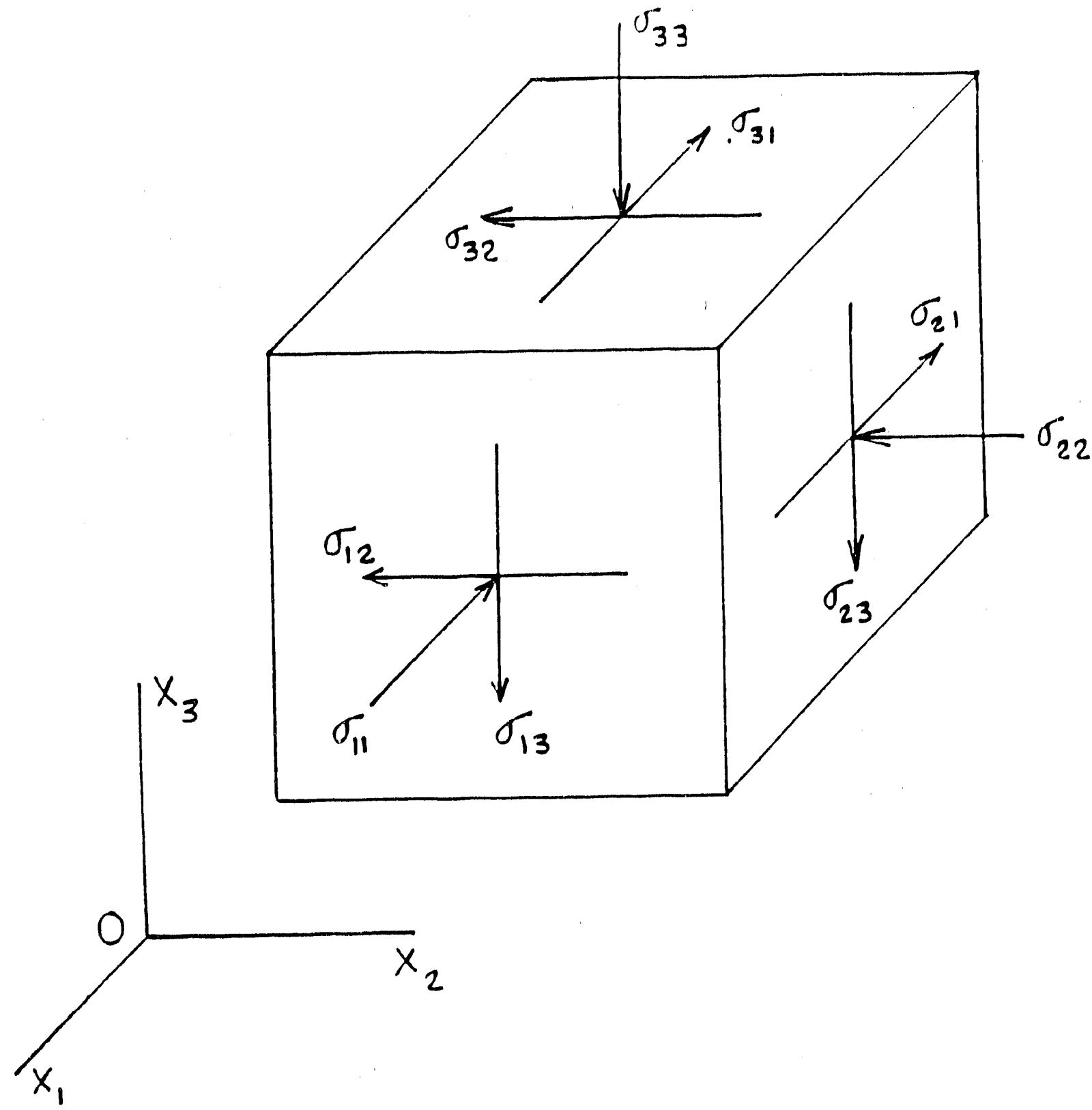

POSITIVE STRESSES

Figure 1. Sign Convention. 


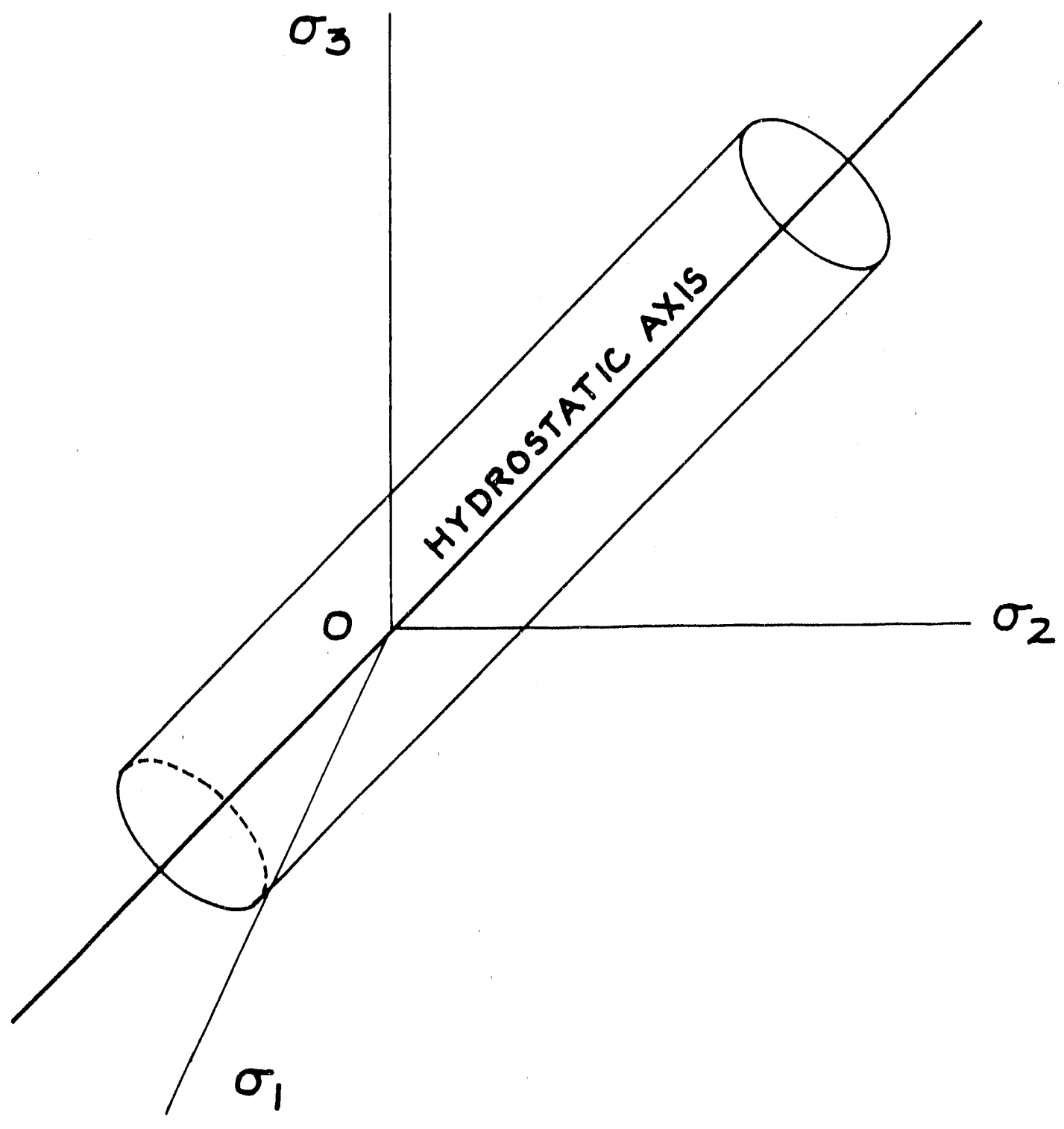

Figure 2. Von Mises Yield Surface. 


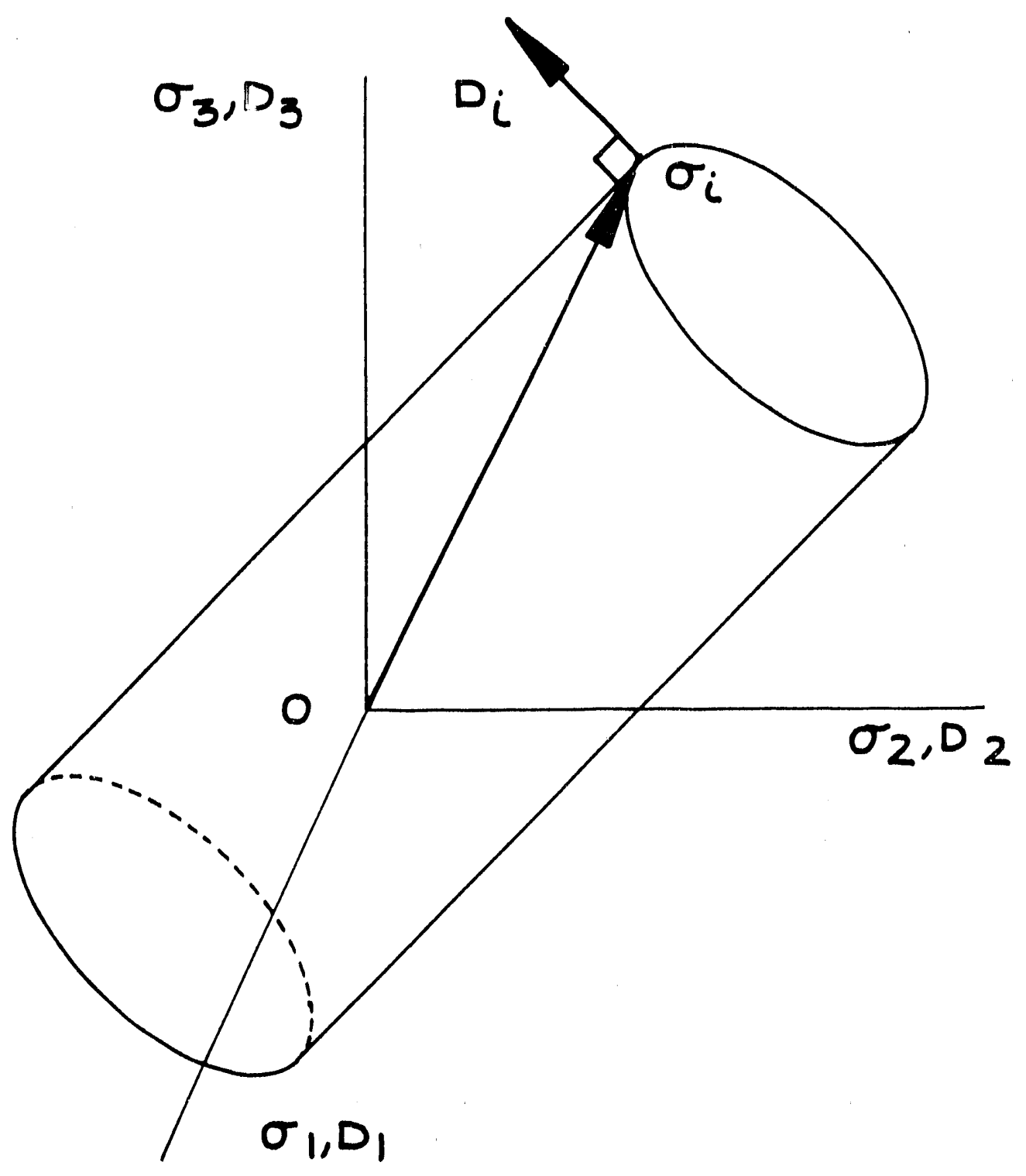

Figure 3. Associated Flow Rule. 


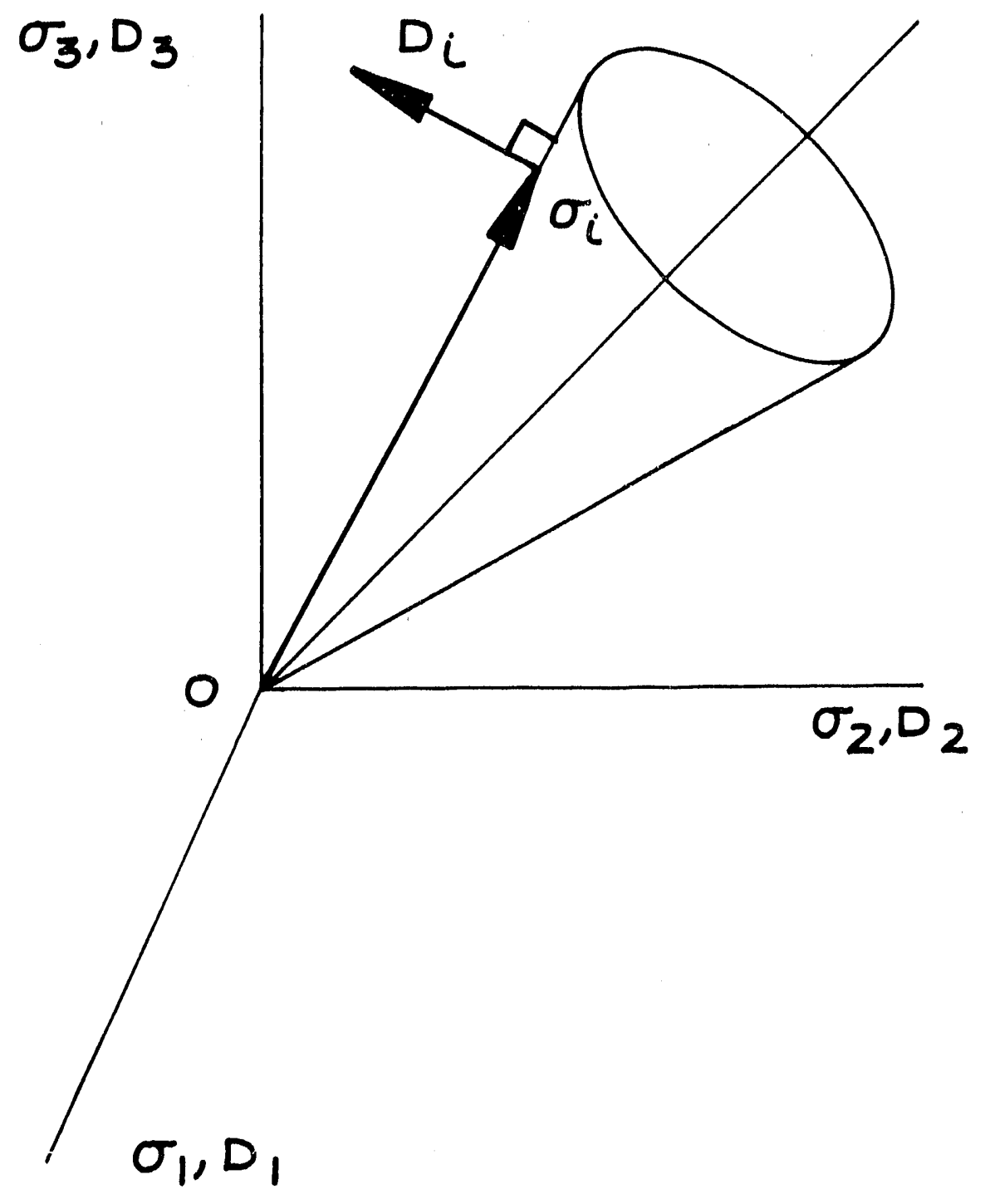

Figure 4. Extended Von Mises Yield Surface. 


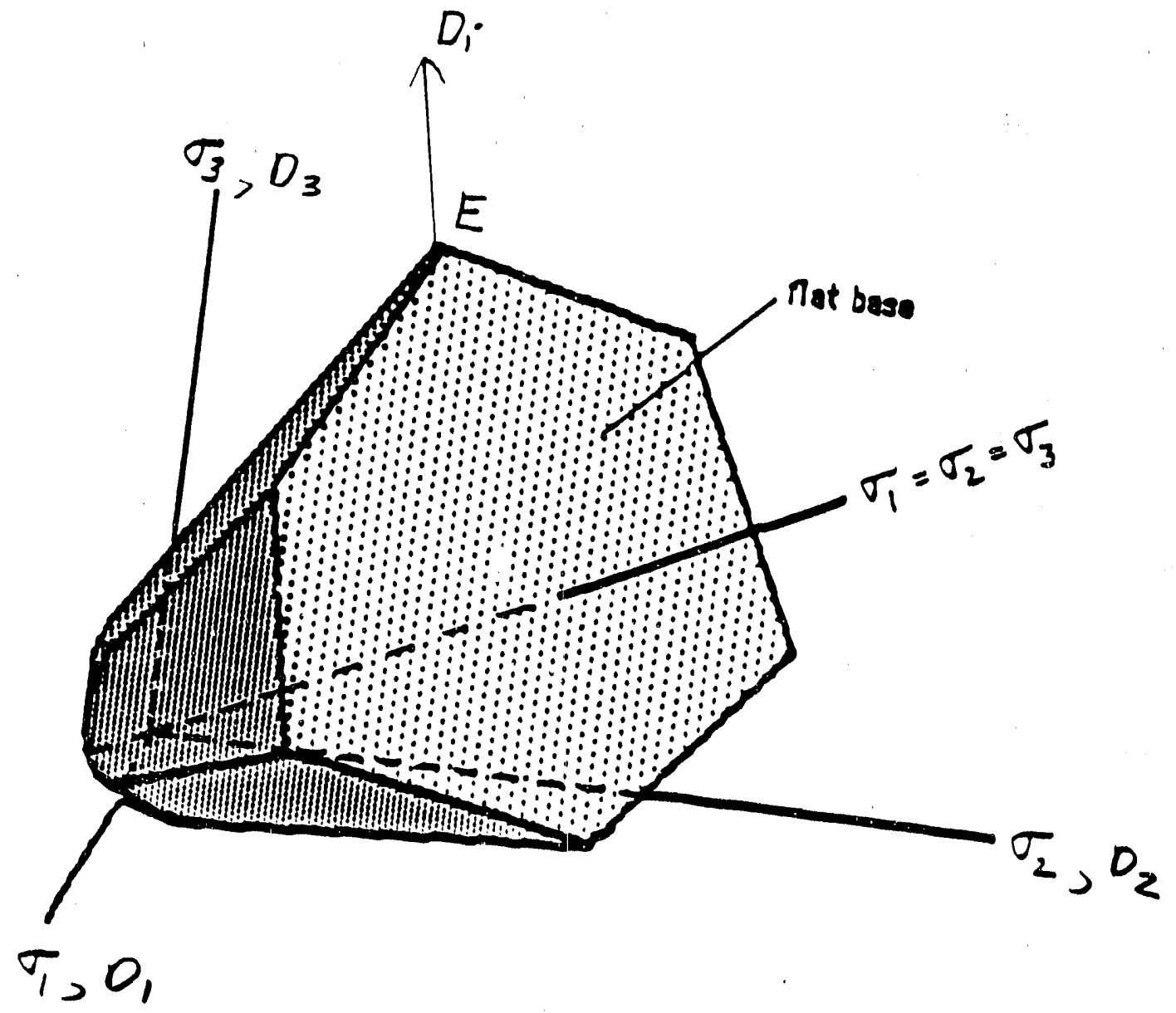

Figure 5. Mohr-Coulomb Yield Surface. 


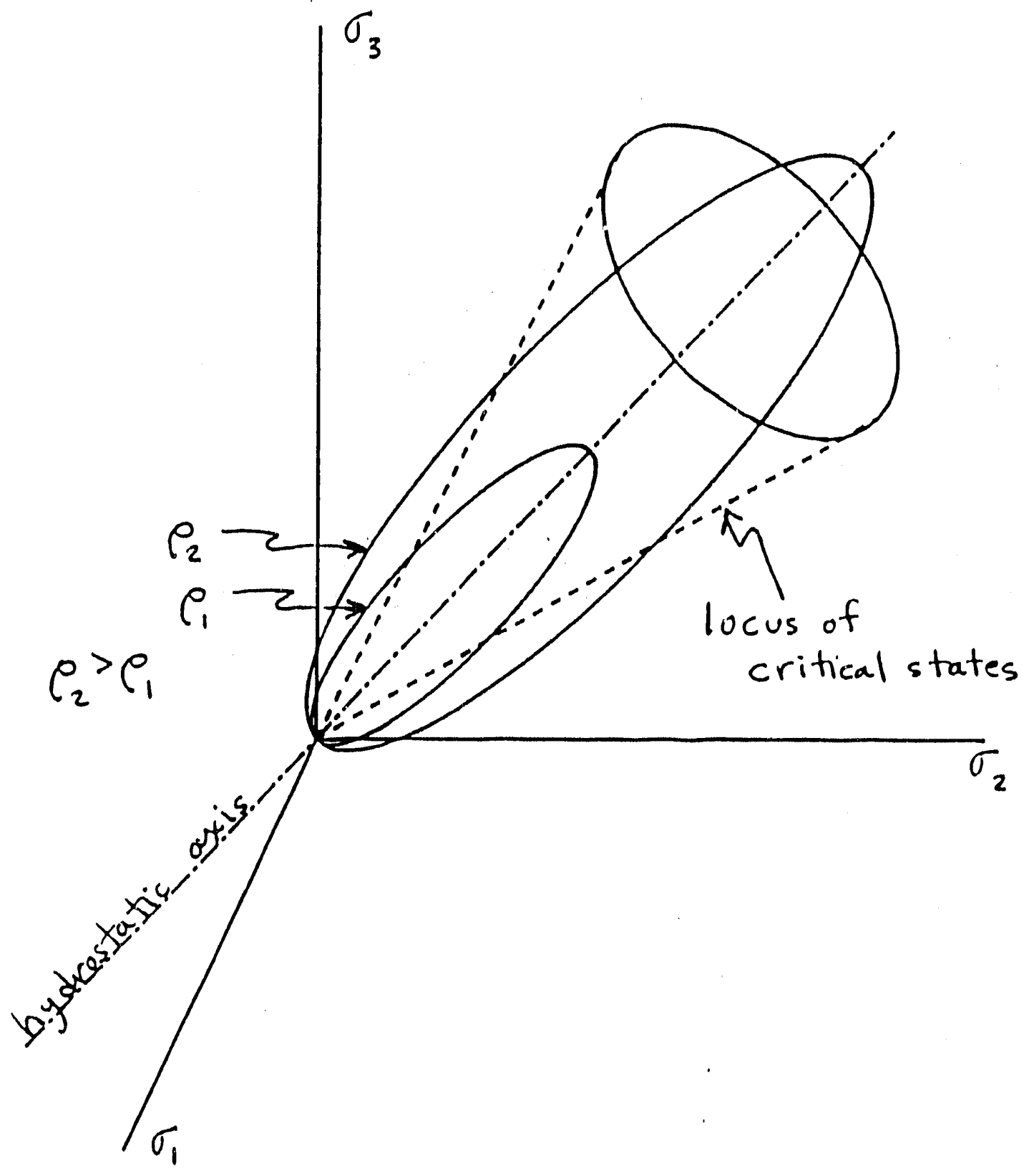

Figure 6. Gray-Stiles Yield Surface. 


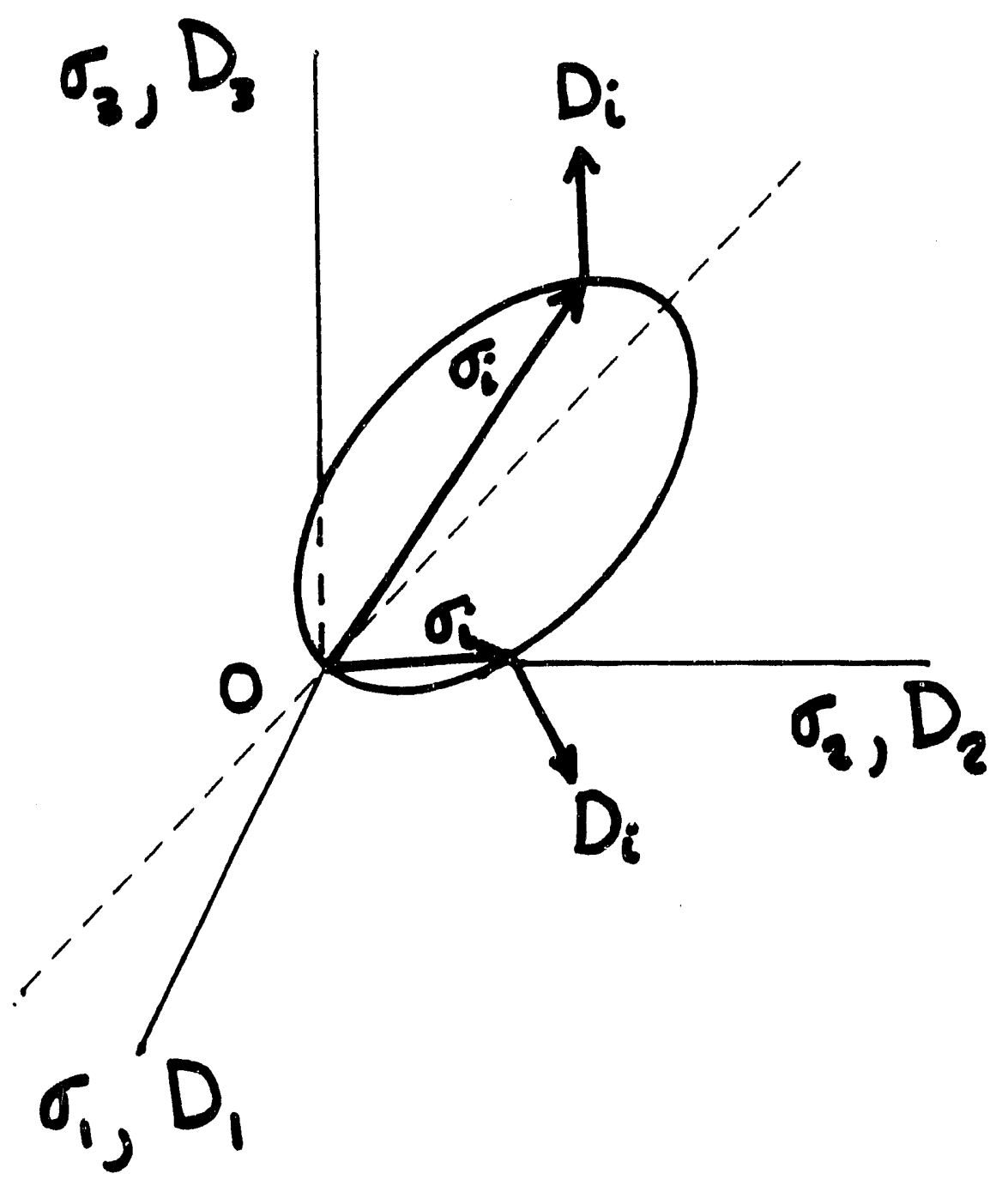

Figure 7. Gray-Stiles Yield surface and Associated Flow Rule. 


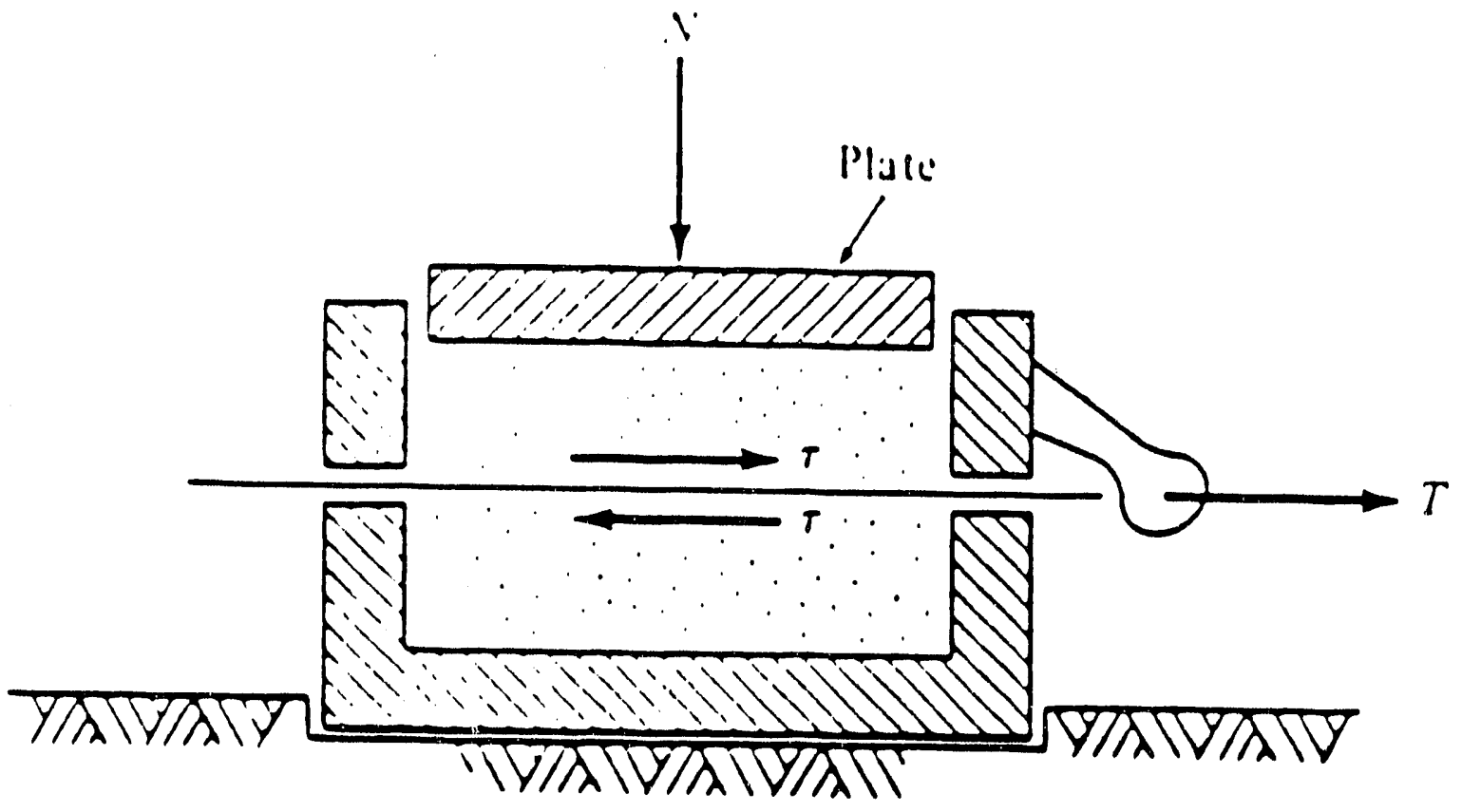

Figure 8. Schematic of a Shear Cell from Cernica (1982). 


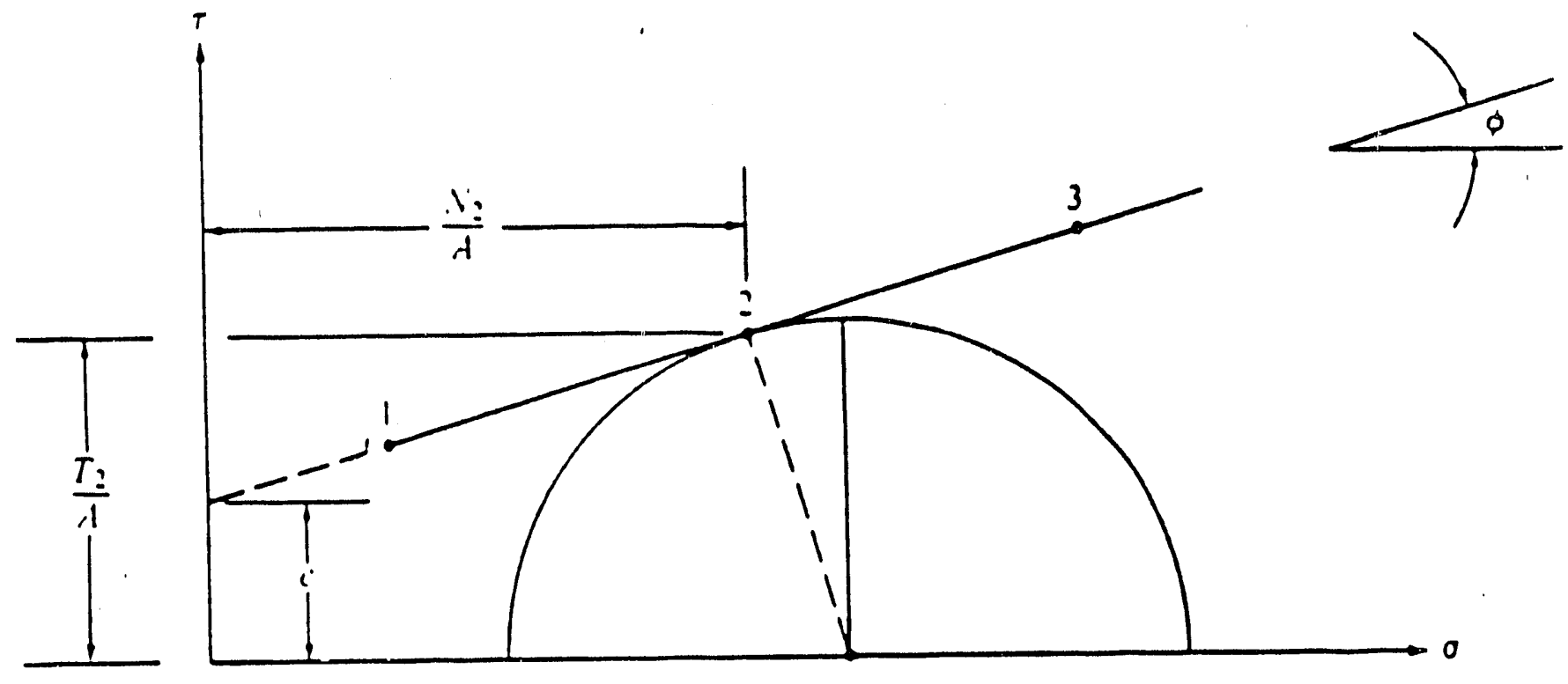

Figure 9. Example Plot of Failure Envelope and Corresponding Mohr circle. 


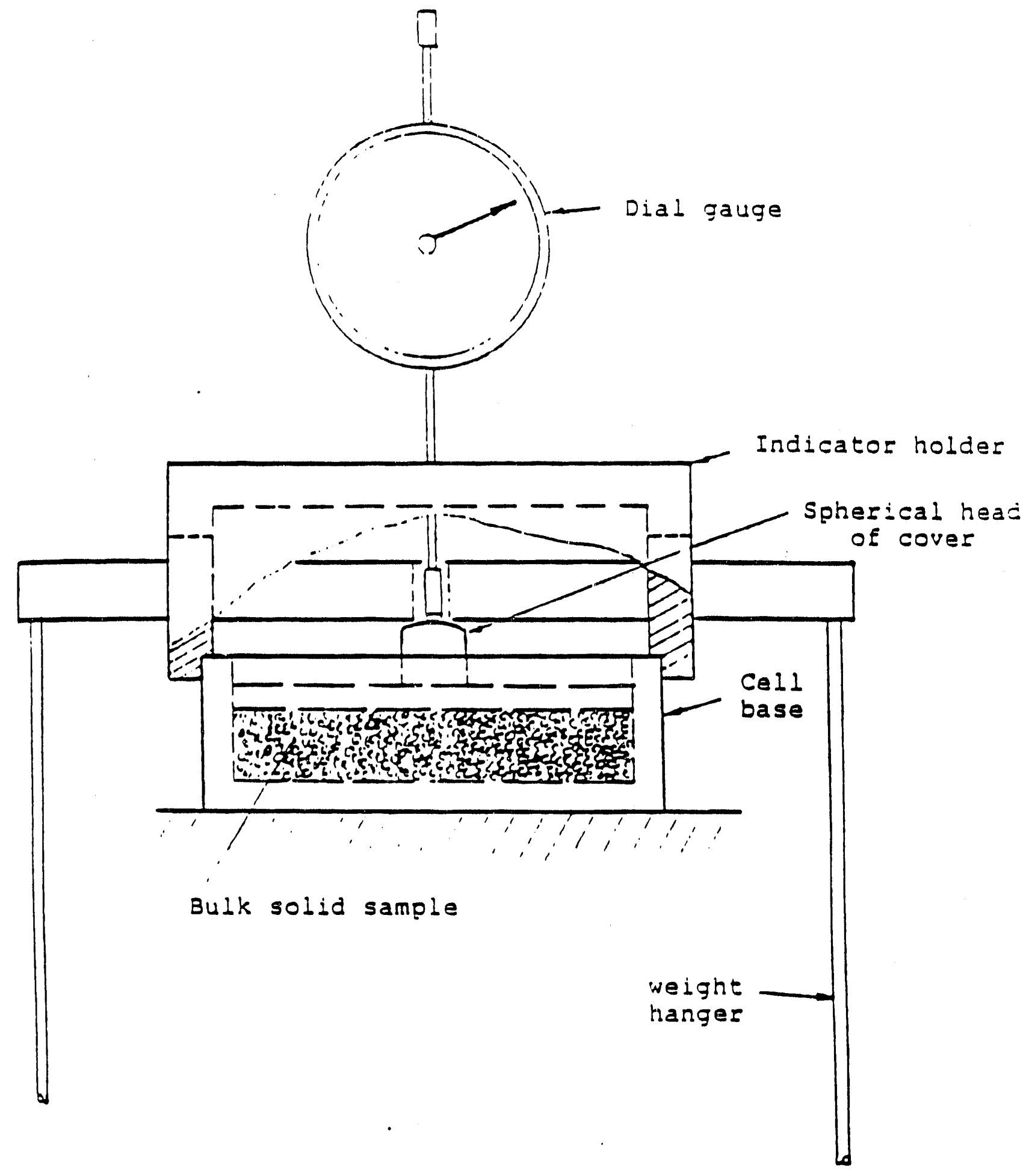

Figure 10. Schematic of Standard Compressibility Tester from Royal (1986). 


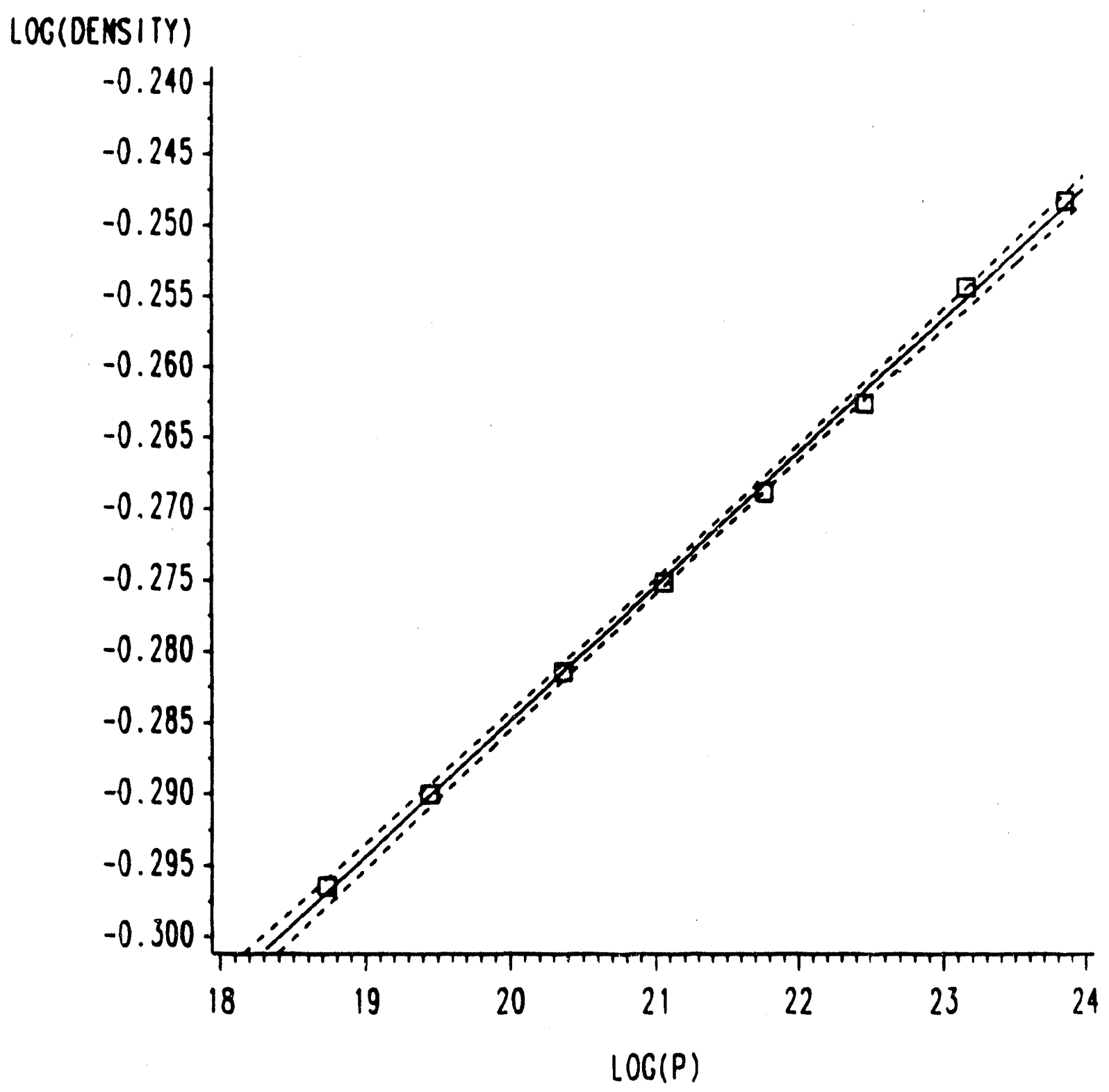

Figure 11. Critical state Pressure, $p$, Versus Density, $\rho$, on Logarithmic Axes for Granular Material No. 1. 


\section{LOG(DENSITY)}

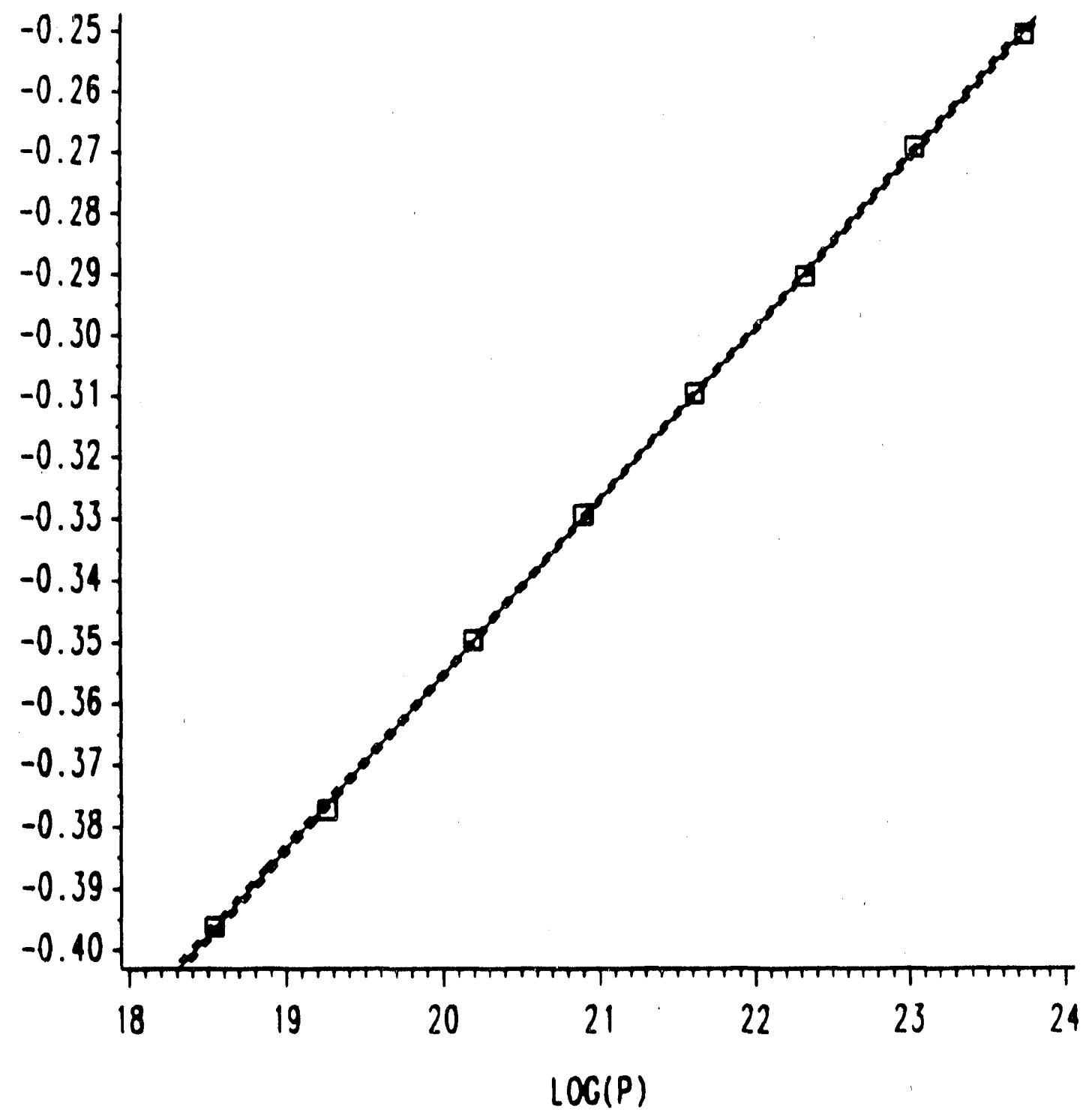

Figure 12. Critical state Pressure, $p$, Versus Density, $\rho$, on Logarithmic Axes for Granular Material No. 2 . 


\section{LOG (DENSITY)}

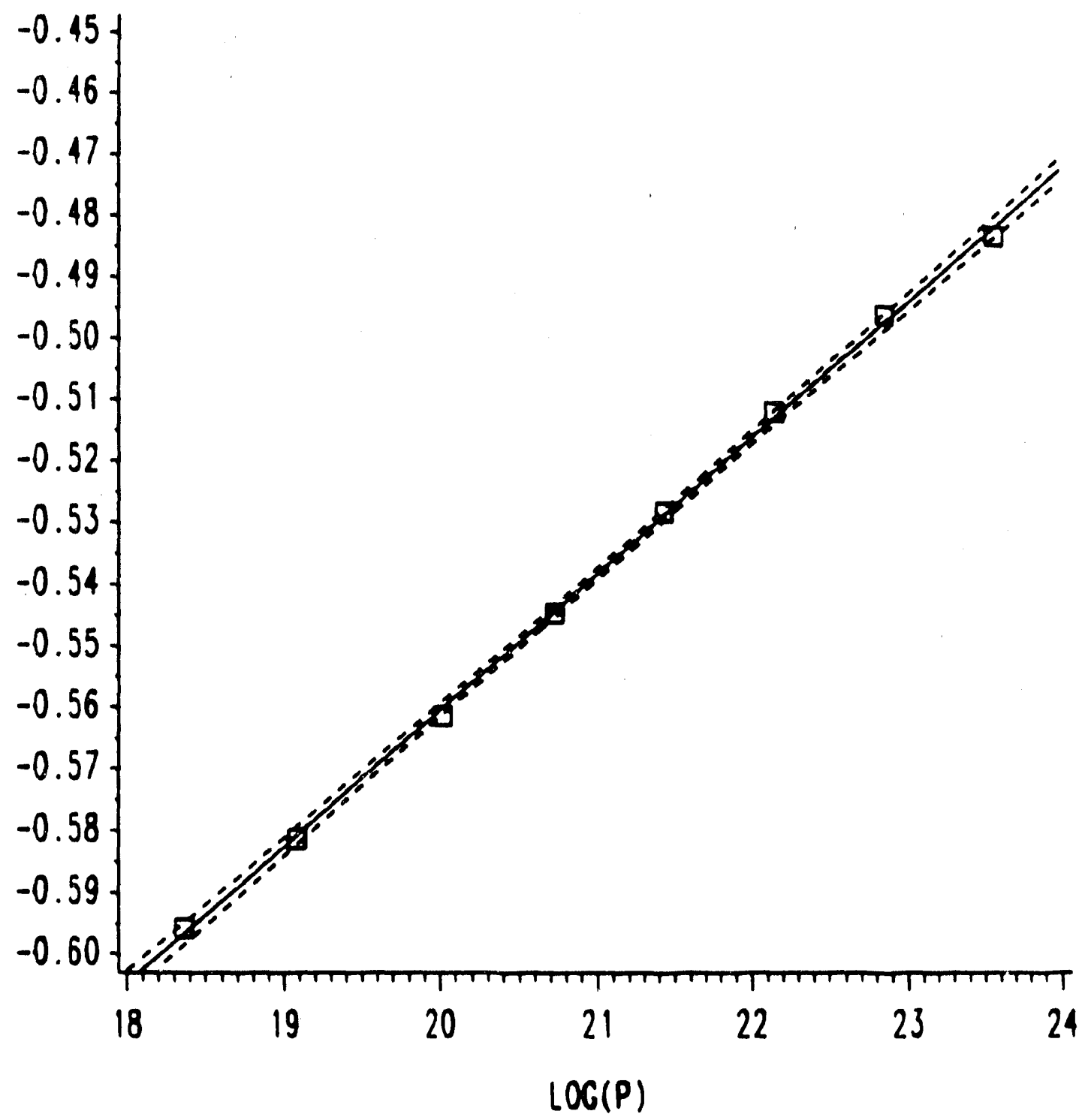

Figure 13. Critical state Pressure, $p$, Versus Density, $\rho$, on Logarithimic Axes for Granular Material No. 3 . 


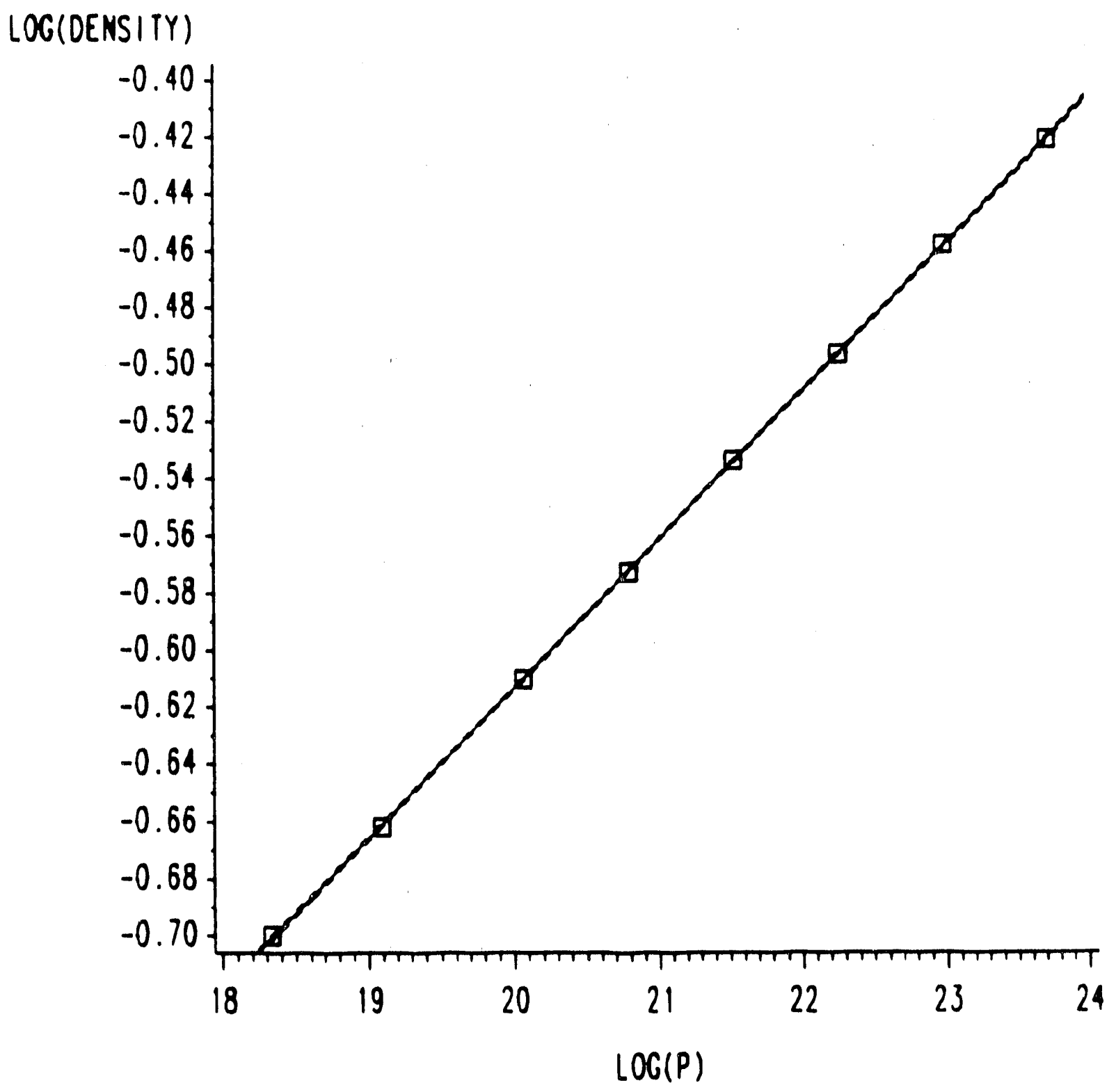

Figure 14. Critical state Pressure, $p$, Versus Density, $\rho$, on Logarithmic Axes for Granular Material No. 4 . 


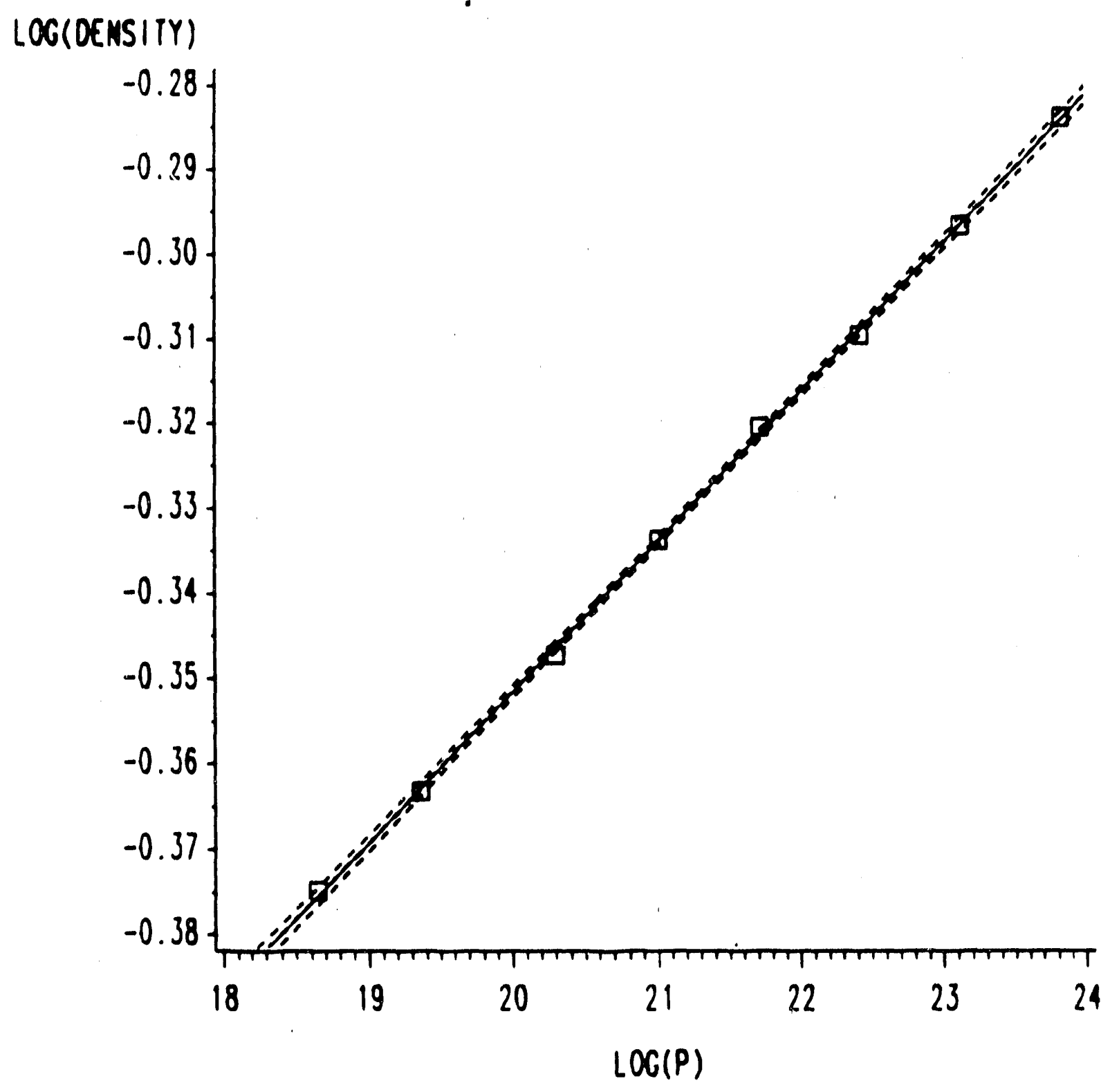

Figure 15. Critical State Pressure, p, Versus Density, $\rho$, on Logarithmic Axes for Granular Material No. 5. 


\section{LOG(DENSITY)}

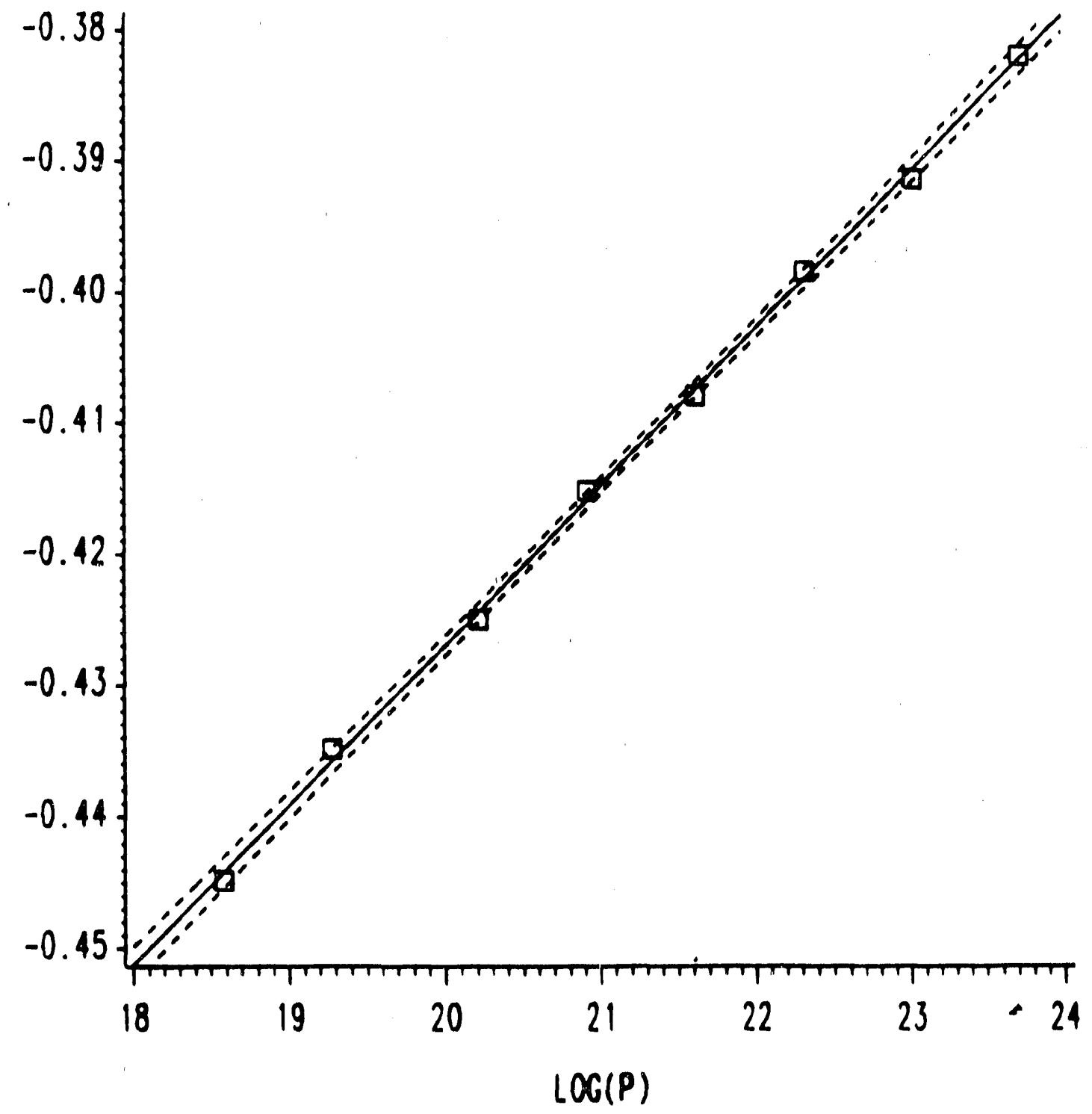

Figure 16. Critical state Pressure, $p$, Versus Density, $\rho$, on Logarithmic Axes for Granualar Material No. 6. 


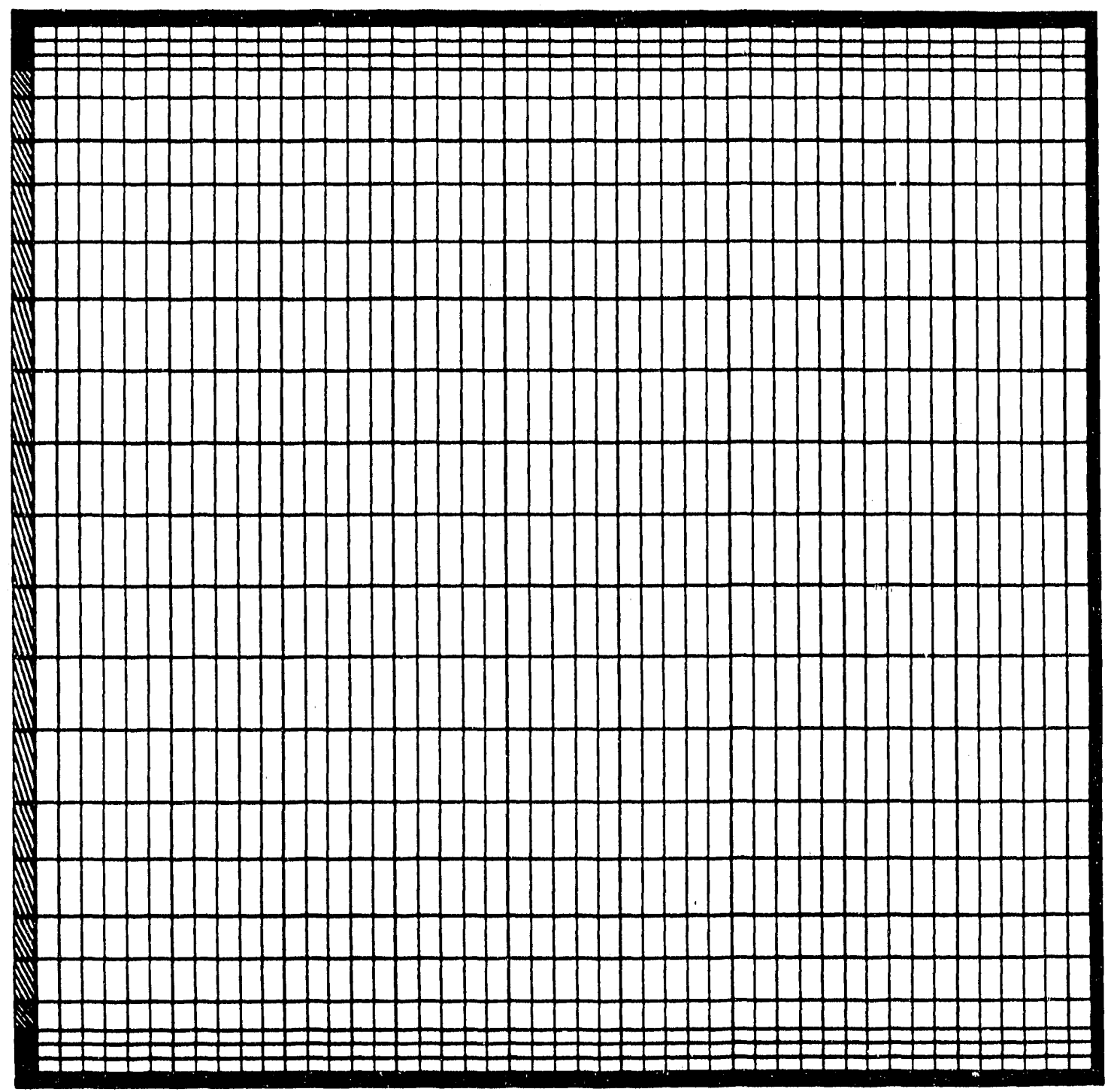

Figure 17. Finite Difference Grid Used for the Von Mises Material Simulations. The vertical scale in this figure is 100 times larger than the horizontal scale. 


\section{VELOCITY VECTORS \\ NITER $=500$}

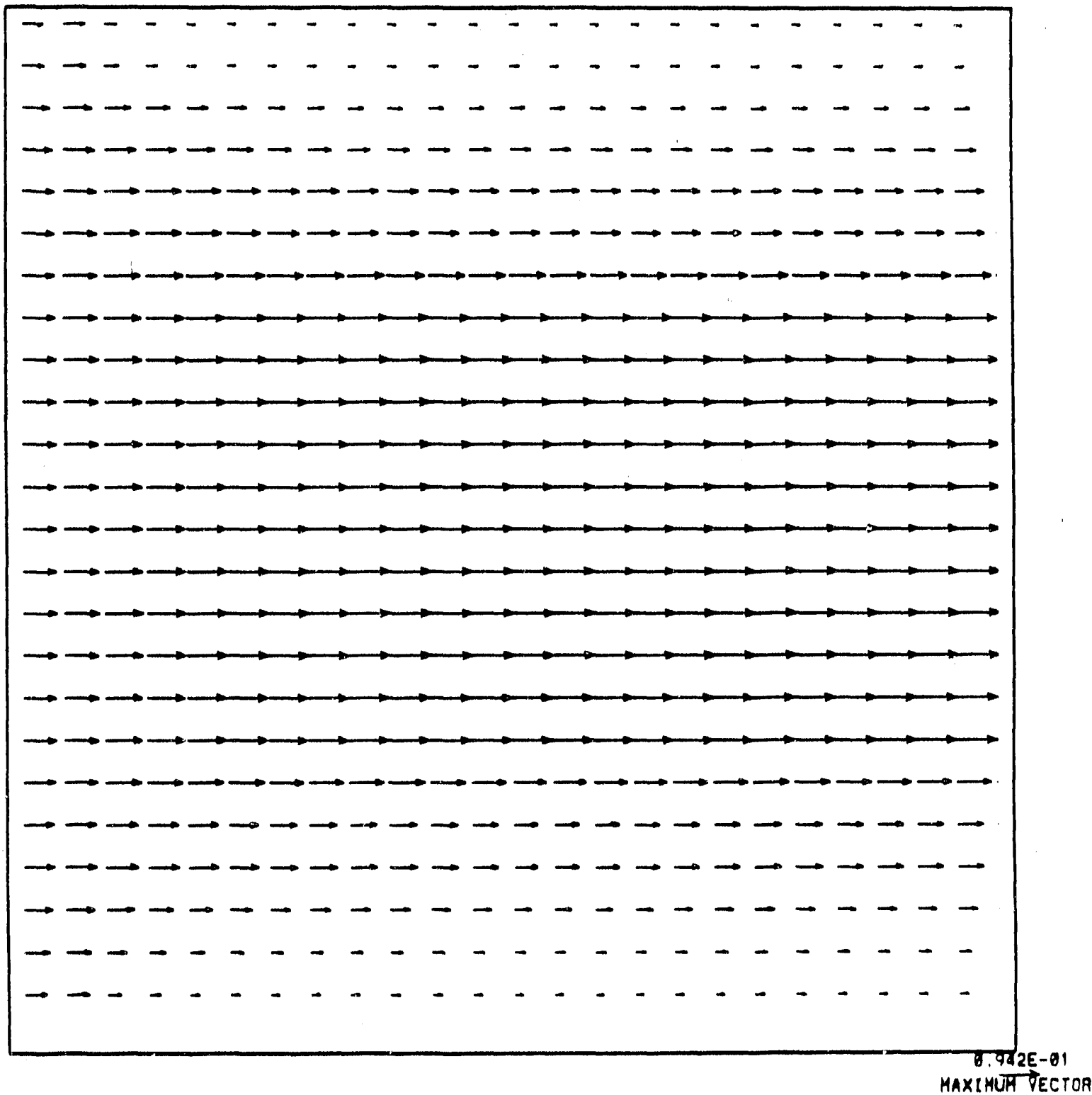

Figure 18. Velocity Vector Plot for the Von Mises Simulation after 500 Iterations. 


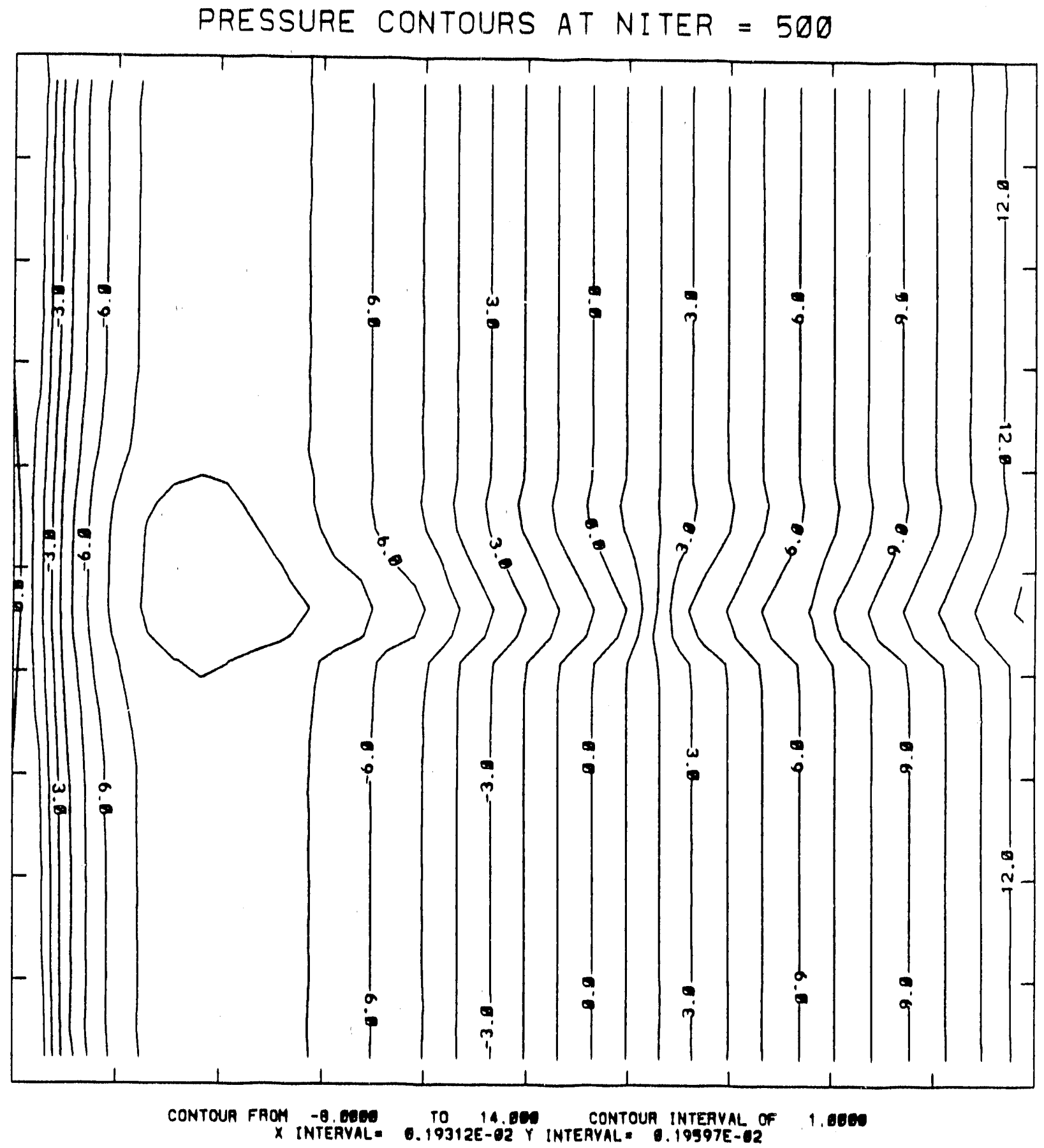

Figure 19. Pressure Contour Plot for the Von Mises Simulation after 500 Iterations. 


\section{VELOCITY VECTORS \\ NITER $=1000$}

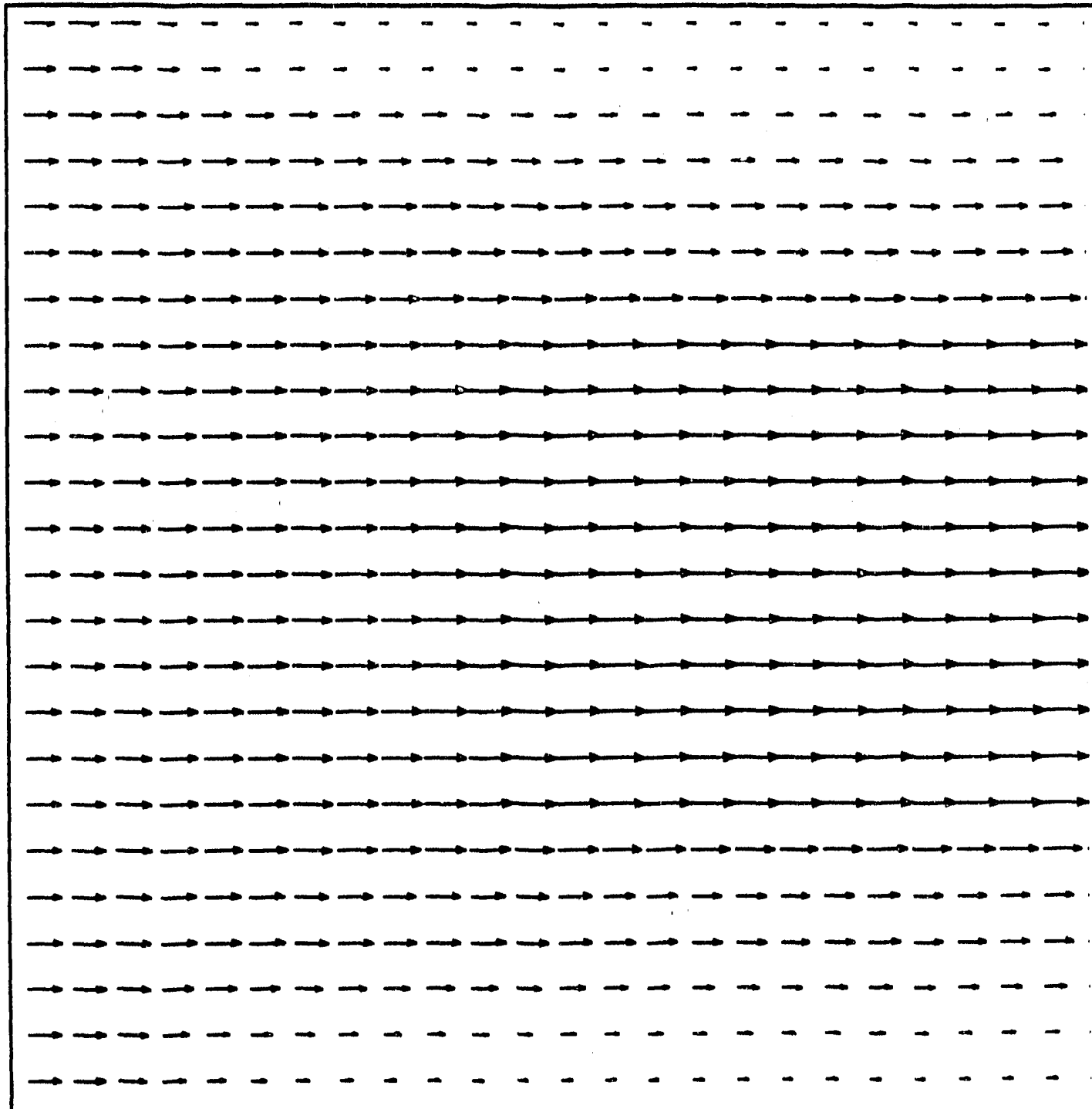

Figure 20. Velocity Vector Plot for the Von Mises Simulation after 1000 Iterations. 


\section{PRESSURE CONTOURS AT NITER $=1000$}

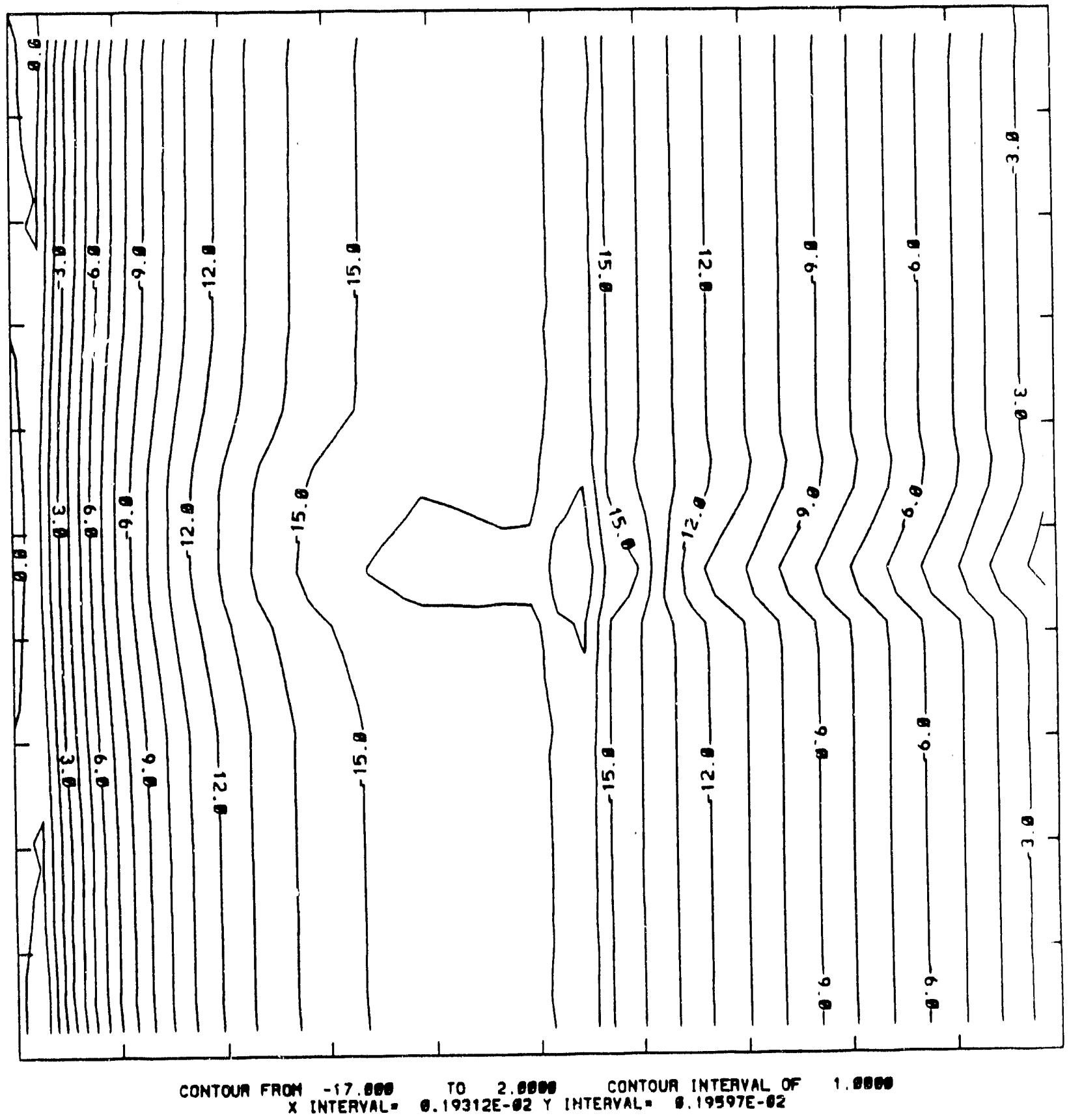

Figure 21. Pressure Contour Plot for the Von Mises Simulation after loúu İterations. 


\section{VELOCITY VECTORS \\ NITER $=10000$}

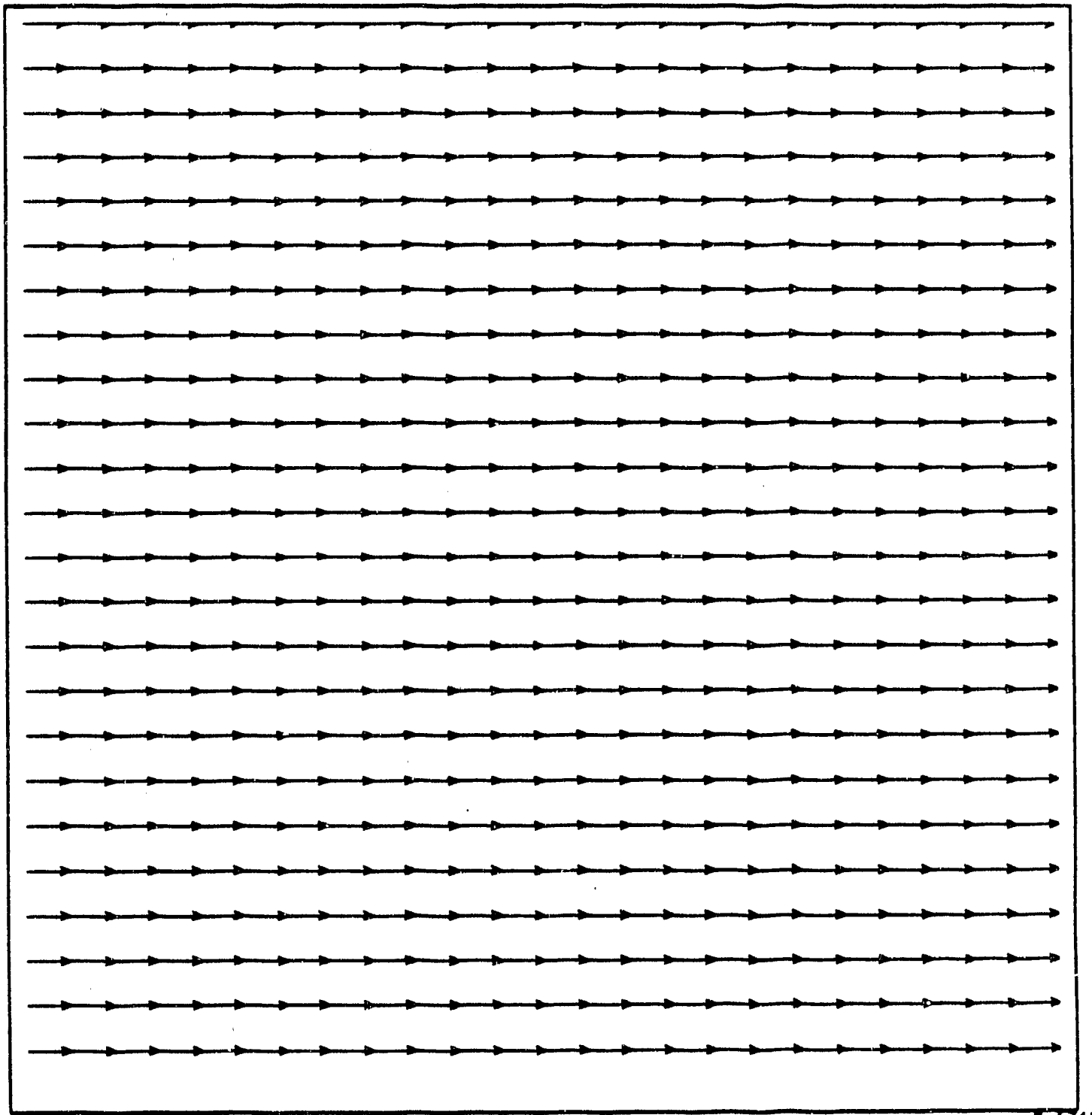

Figure 22. Velocity Vector Plot for the Von Mises Simulation after 10,000 Iterations. 
PRESSURE CONTOURS AT NITER $=10000$

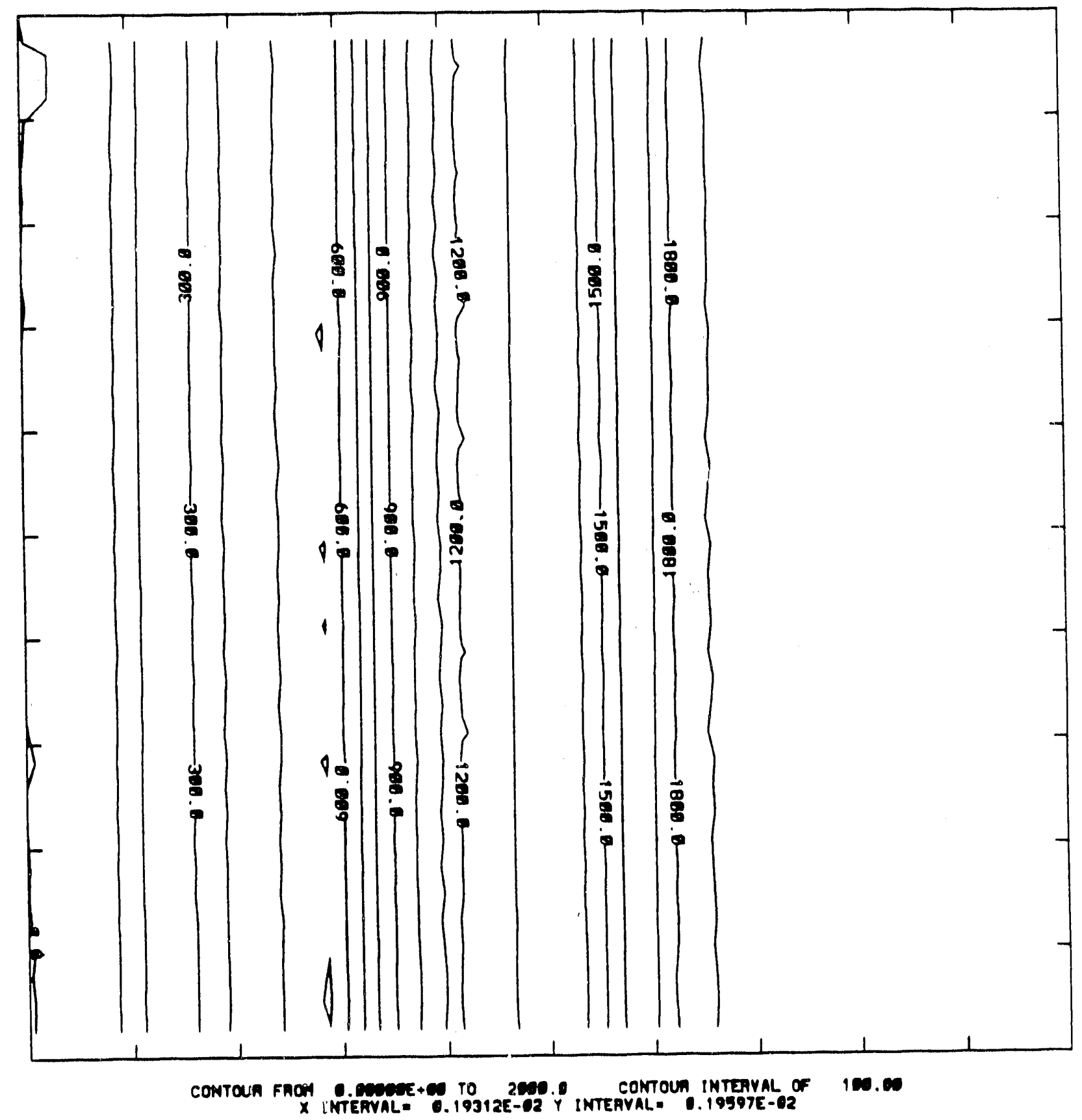

Figure 23. Pressure Contour Plot for the Von Mises Simulation after 10,000 Iterations. 


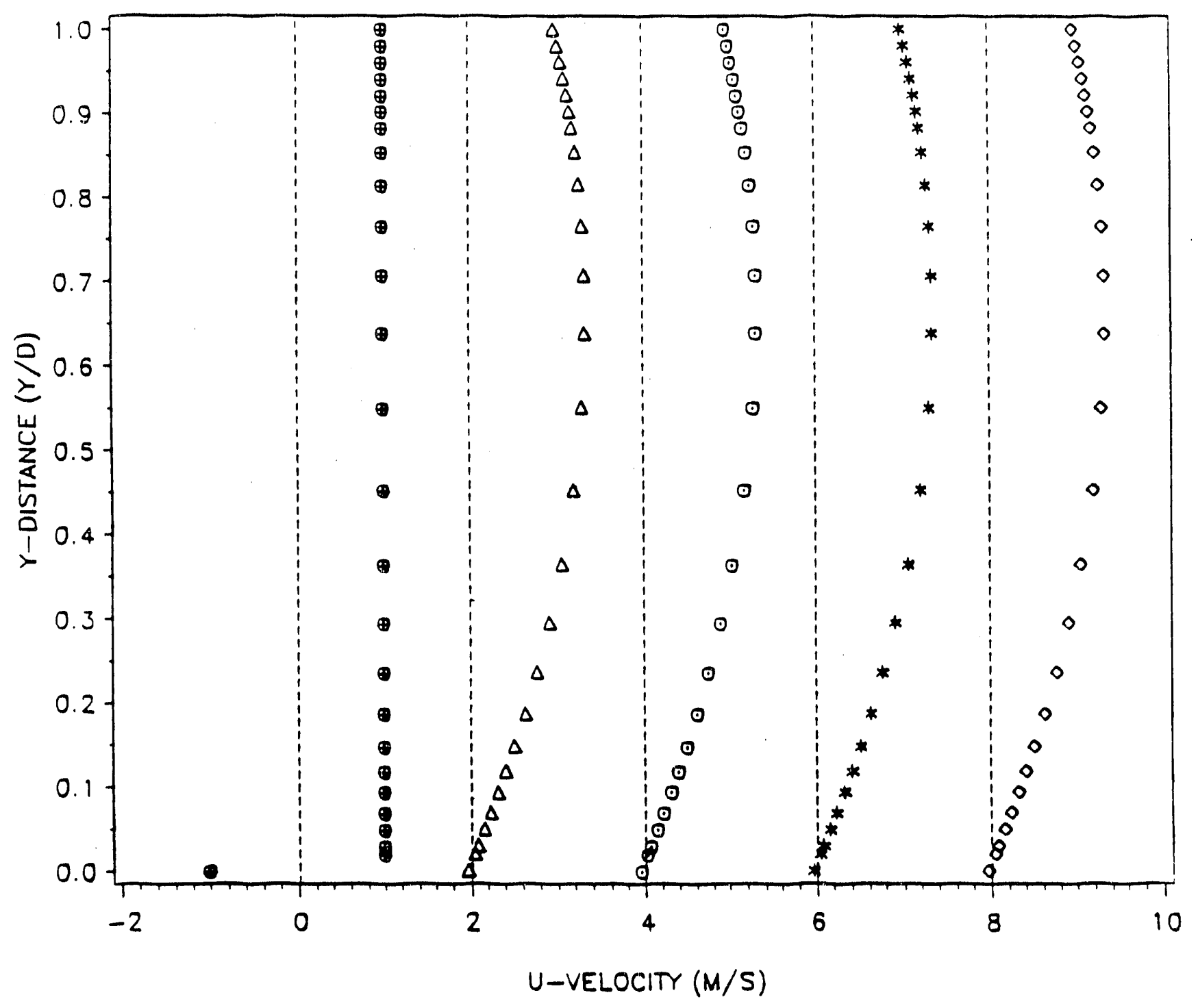

Figure 24. Velocity profiles for Newtonian Poiseuille-Couette Simulation after 1000 Iterations. 


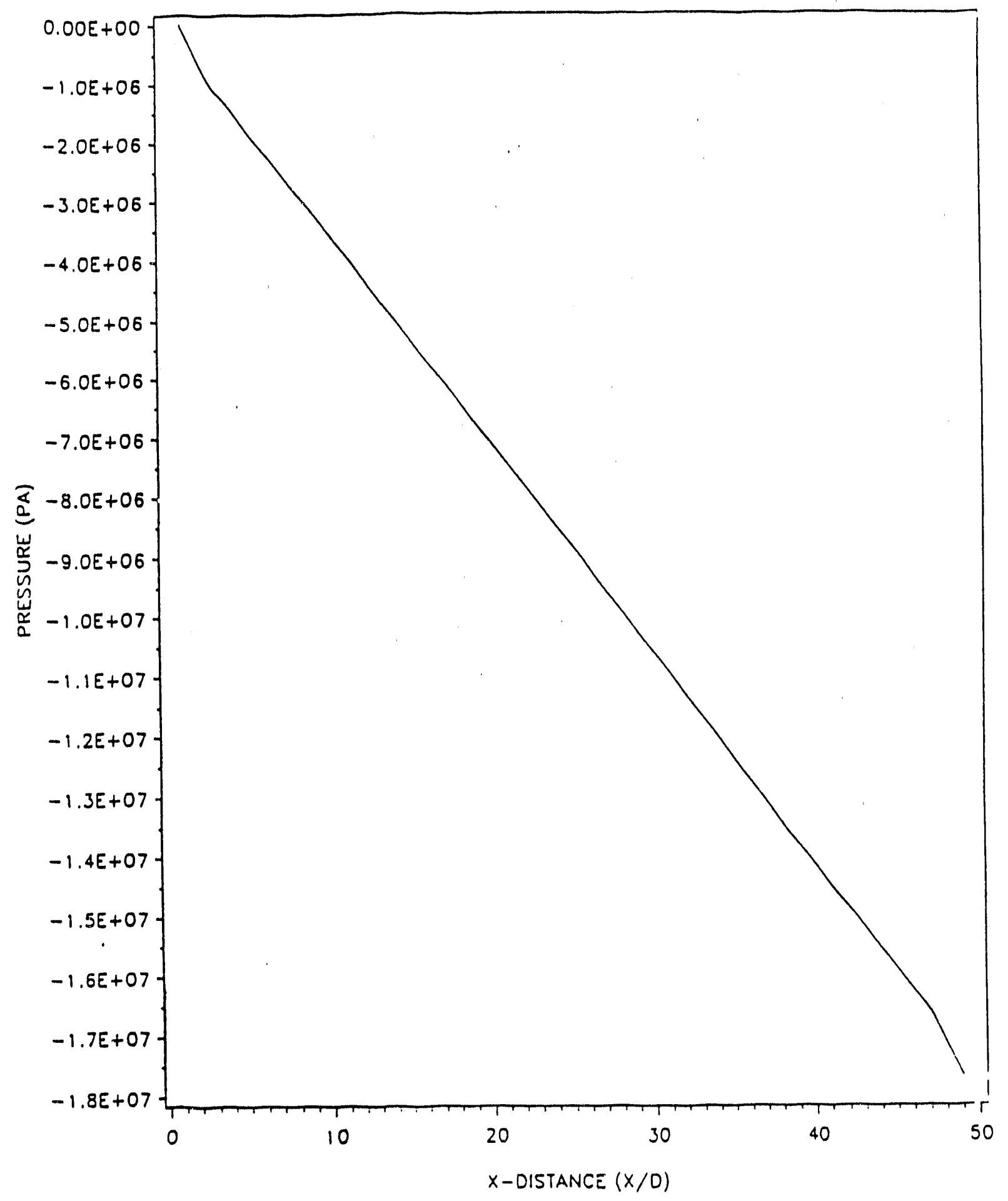

Figure 25. Midline Pressure for Newtonian Poiseuille-Couette Simulation after 1000 Iterations. 


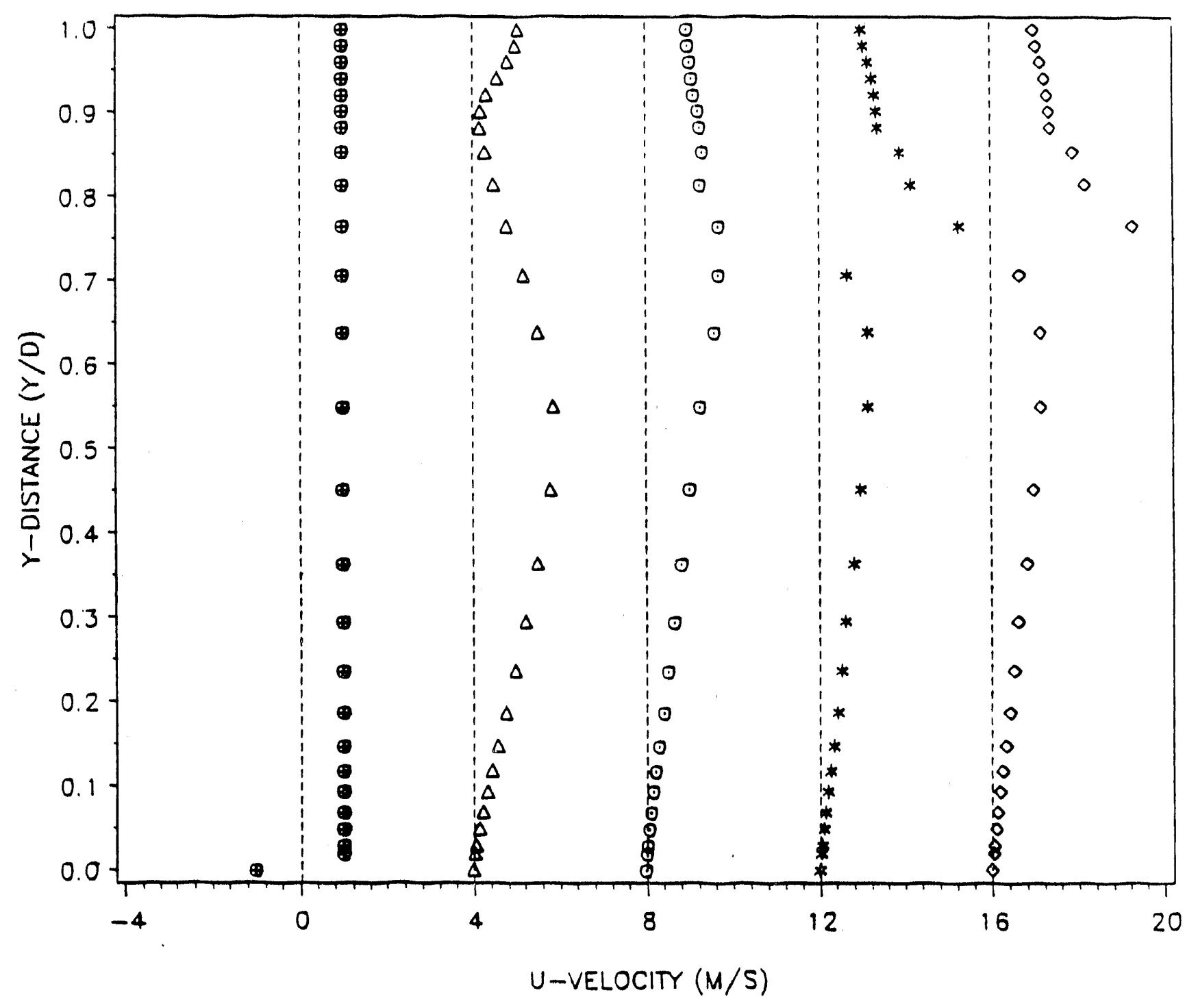

Figure 26. Velocity Profiles for Newtonian/Von Mises/Inviscid Poiseuille-couette simulation. 


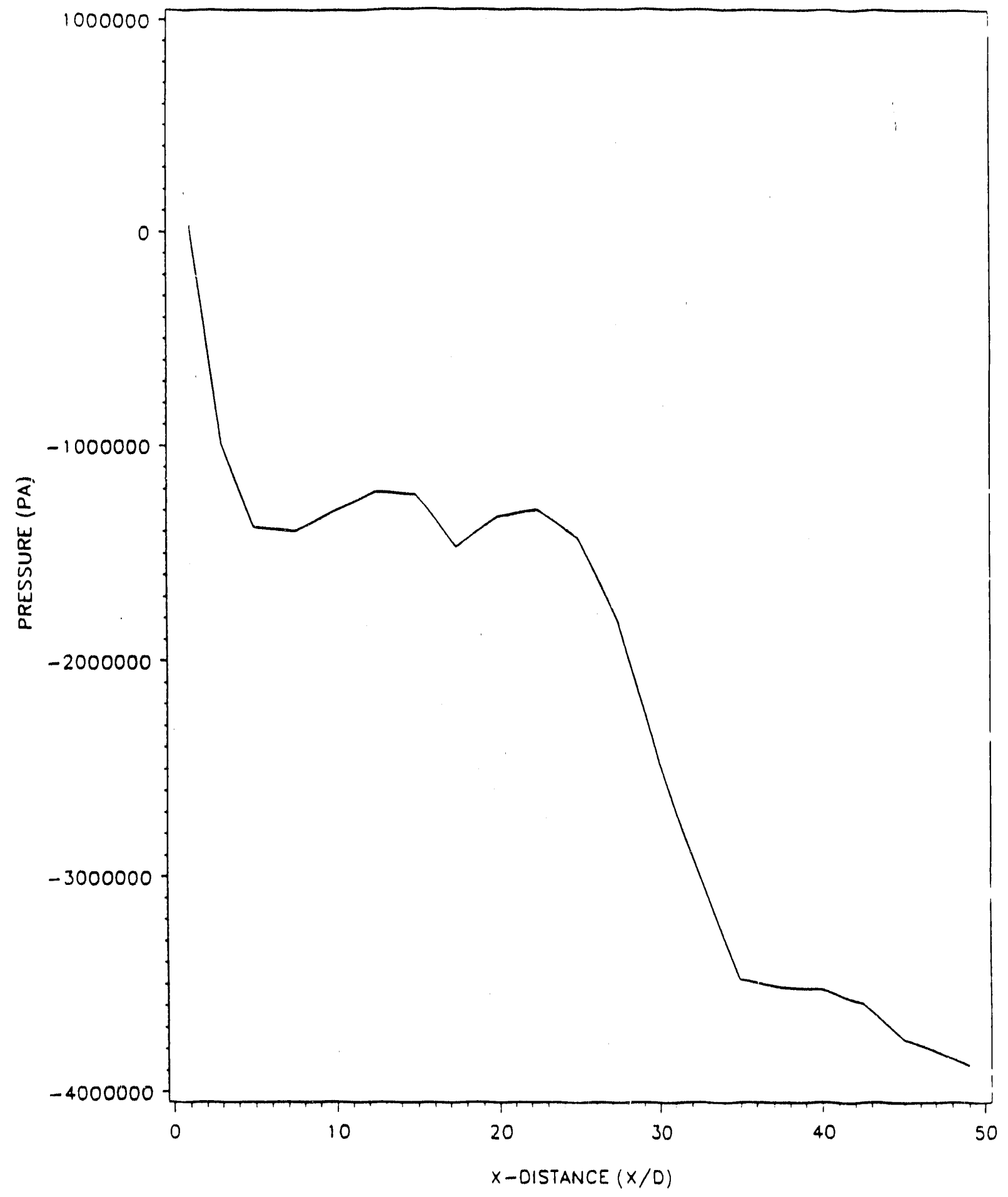

Figure 27. Midline Pressure for Newtonian/Von Mises/Inviscid Poiseuille-Couette Simulation. 


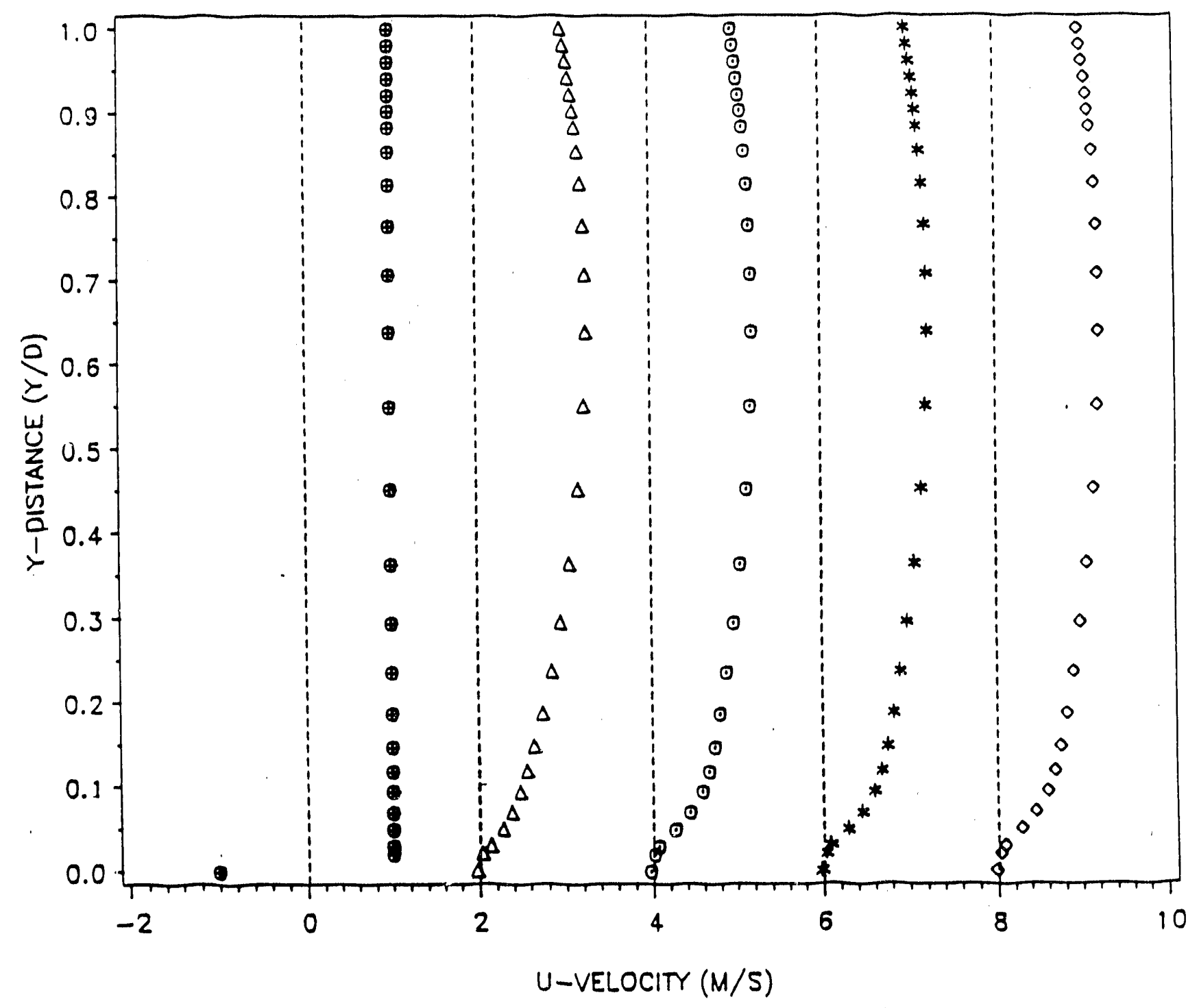

Figure 28. Velocity Profiles for Newtonian/Von Mises/Bounded Viscosity poiseuille-couette simulation. 


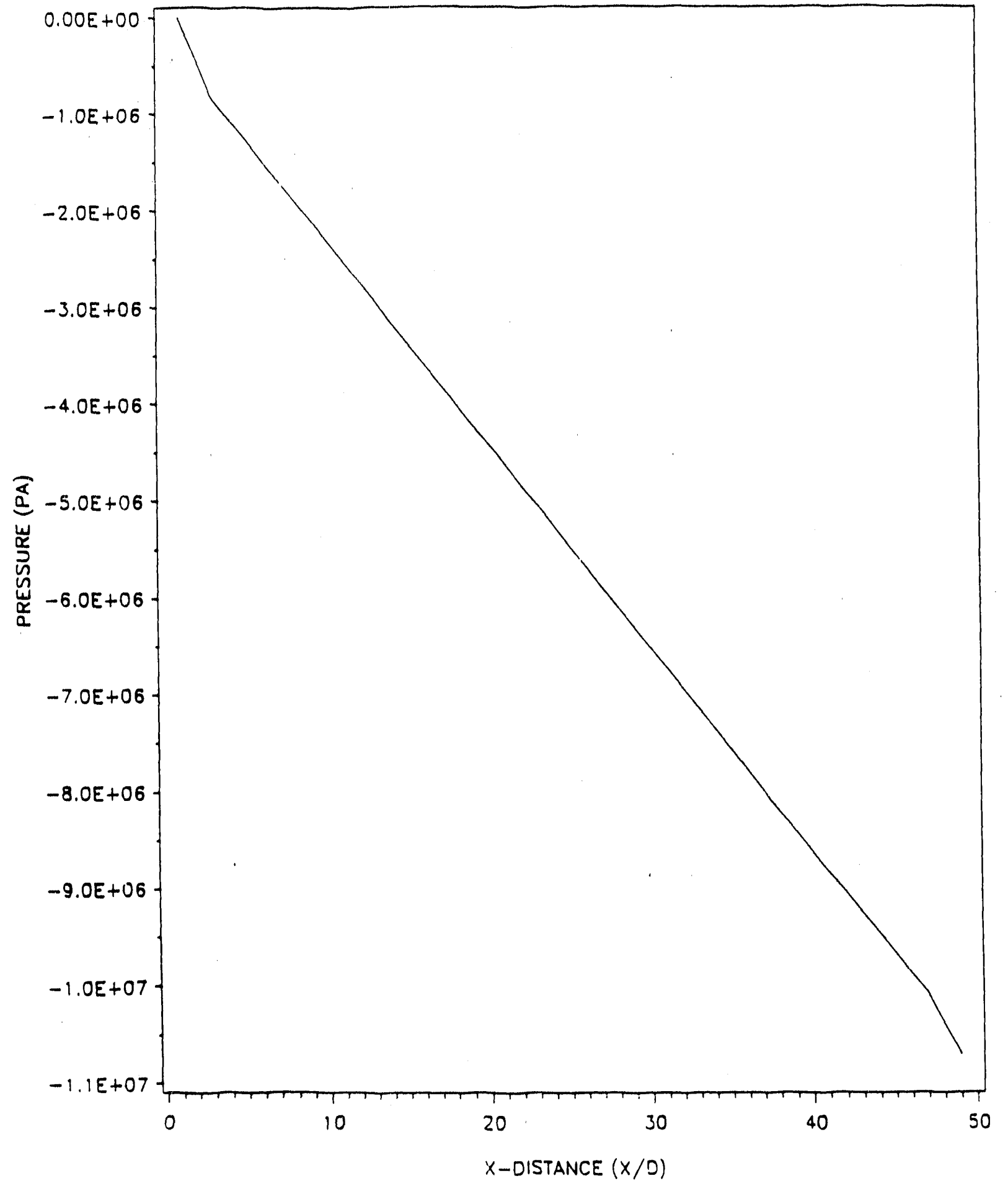

Figure 29. Midline Pressure for Newtonian/Von Mises/Bounded Viscosity Poiseuille-Couette Simulation. 


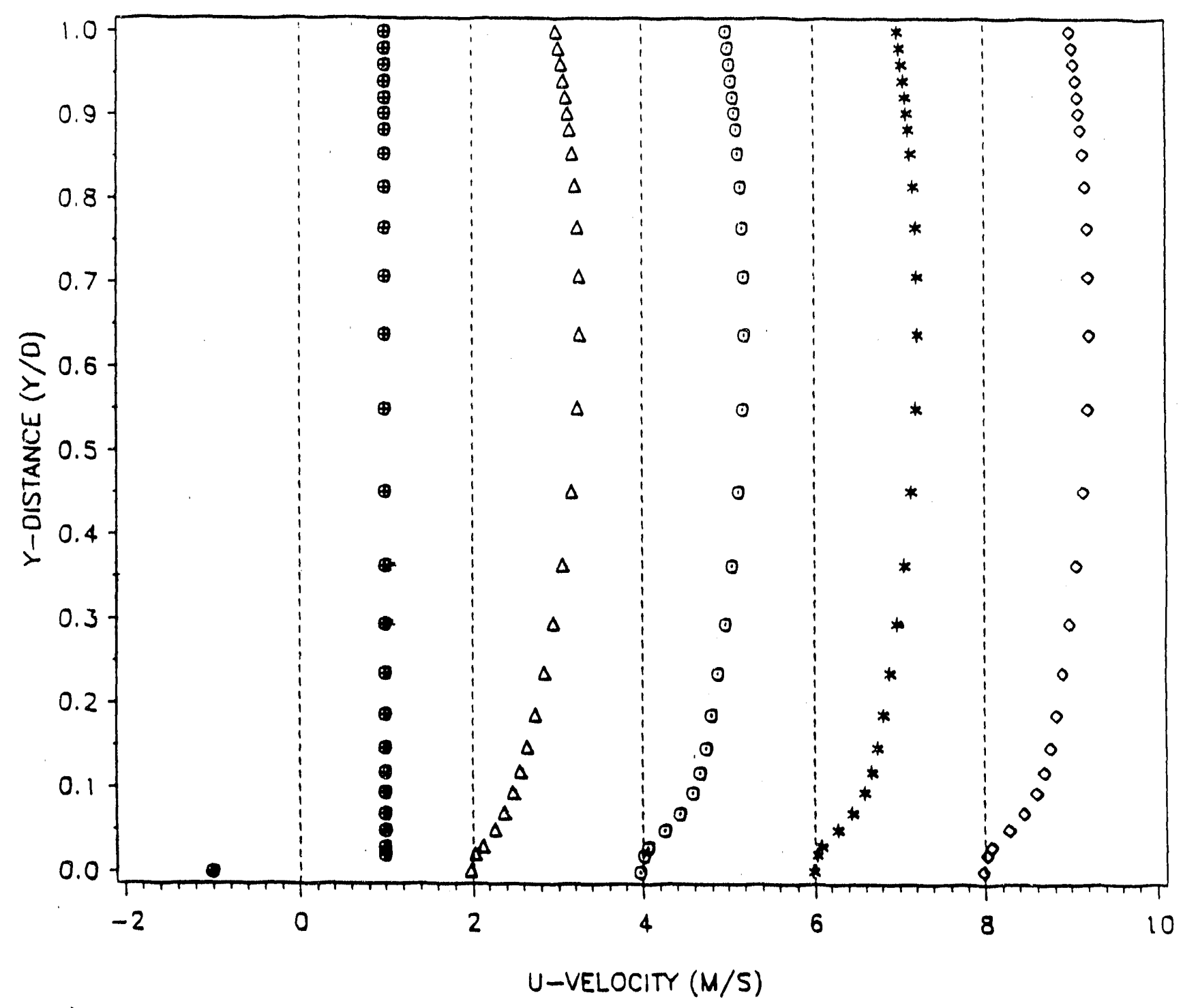

Figure 30. Velocity Profiles for Newtonian/Von Mises/Bounded Viscosity Plus Kinetic Effects Poiseuille-couette Simulation. 


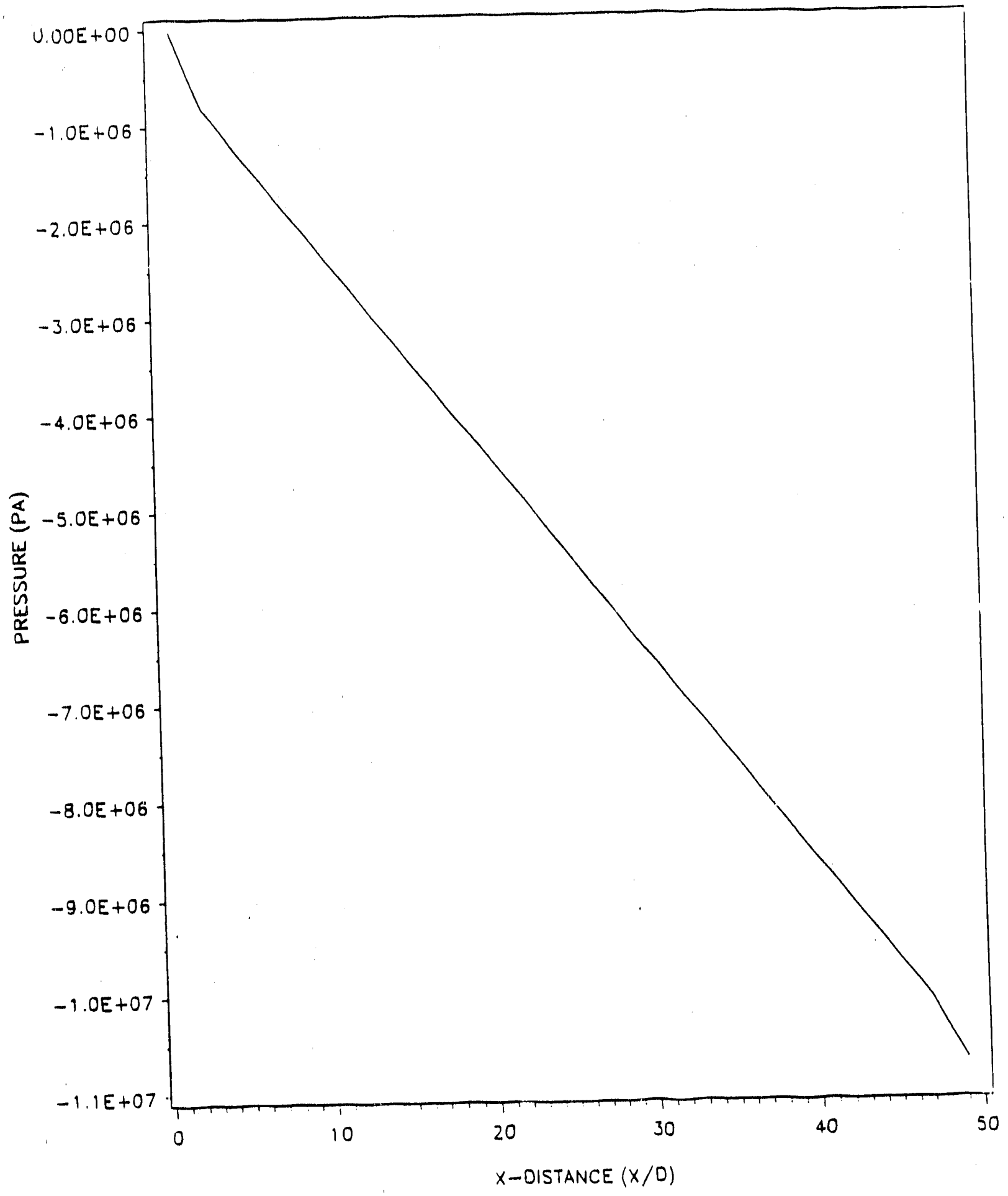

Figure 31. Midline Pressure for Newtonian/Von Mises/Bounded Viscosity Plus Kinetic Effects Poiseuille-couette simulation. 


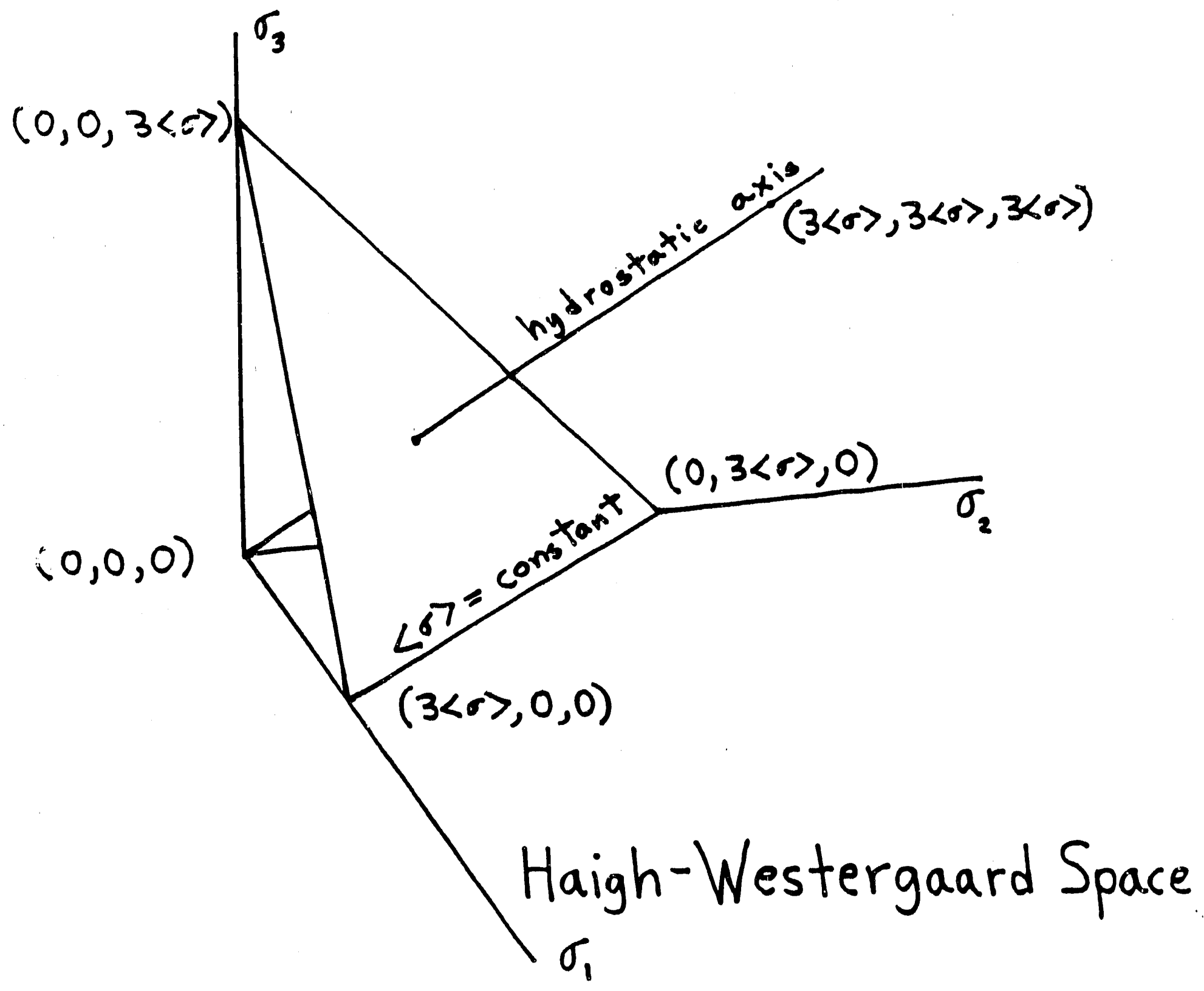

Figure 32. Features of Haigh-Westergaard Space. 


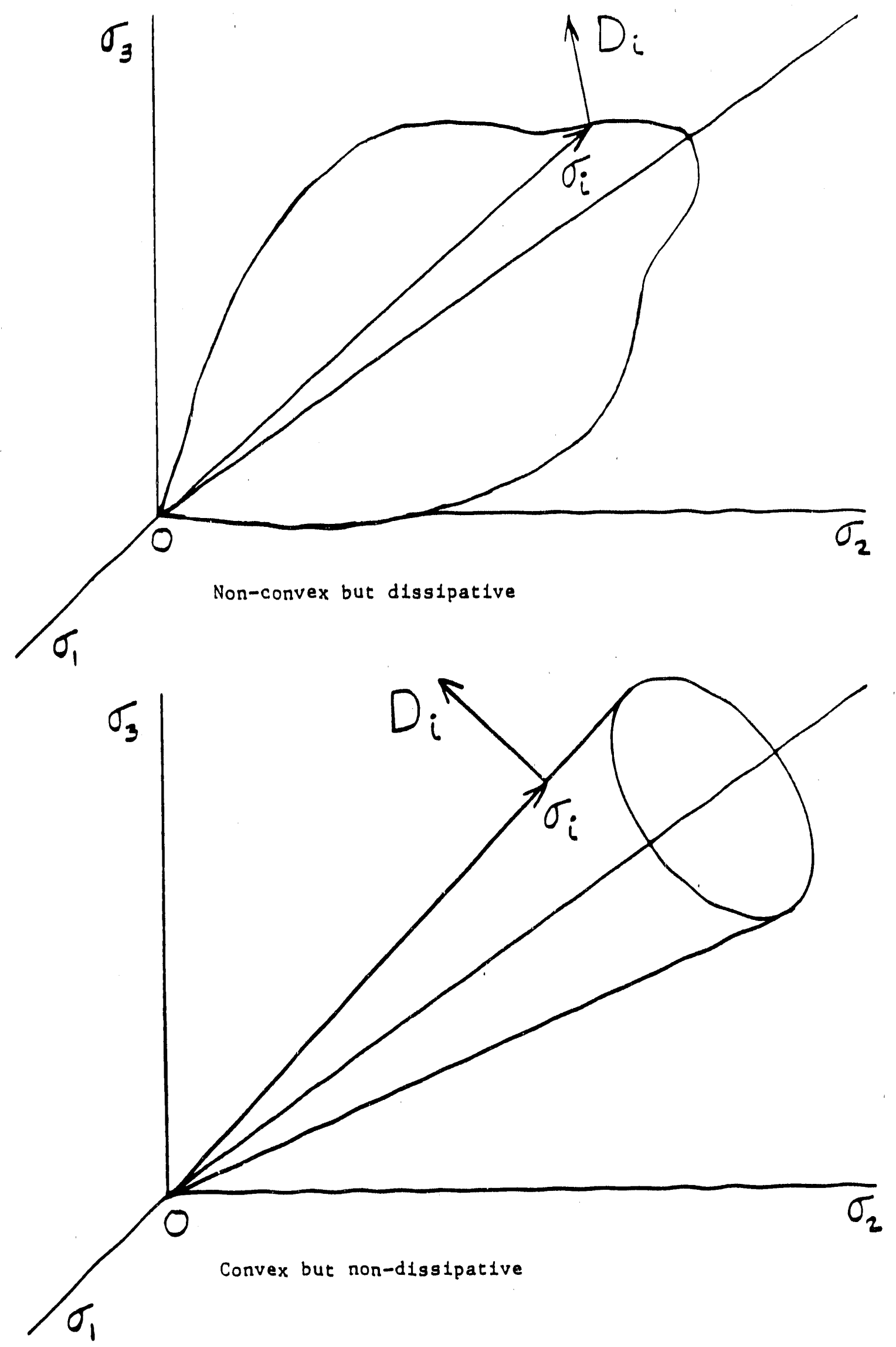

Figure 33. Distinction between Convexity and Dissipativeness. 


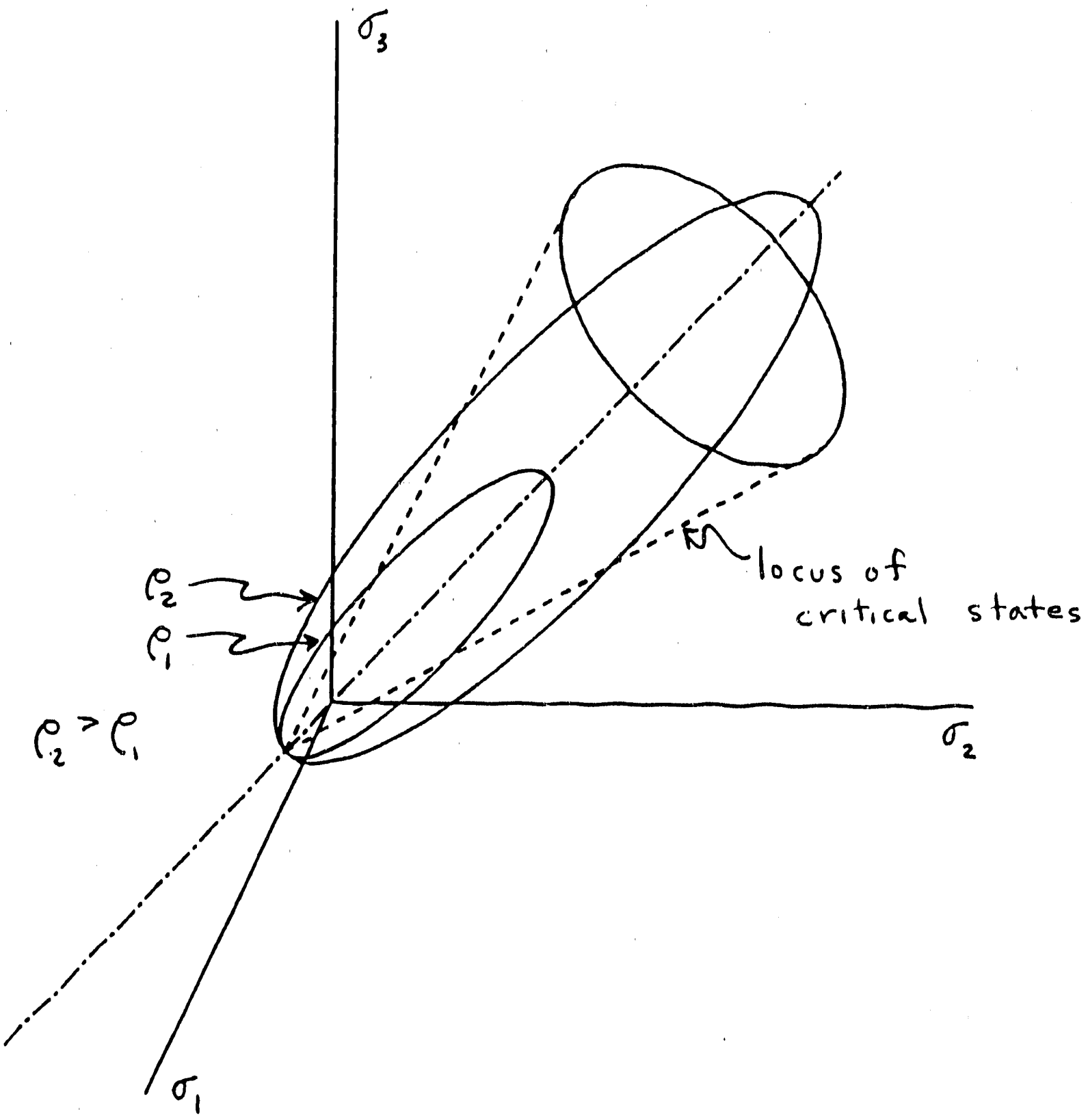

Figure 34. Gray-stiles Yield Surface for Cohesive Material. 
YIELD SURFACE F4

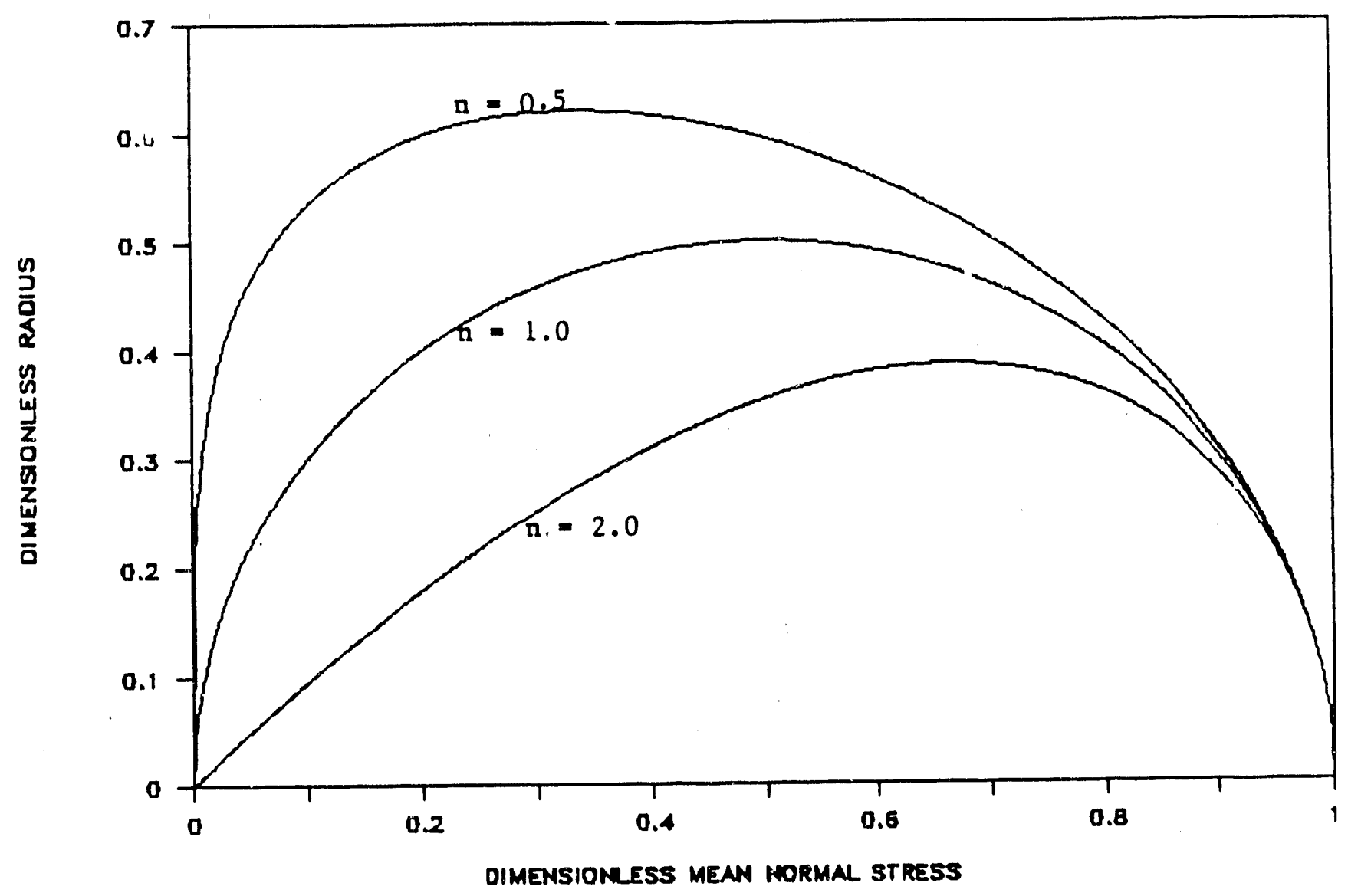

Figure 35. Dime; sionless Radius of Yield surface 4 for $n=0.5$, $1.0,2.0$. 


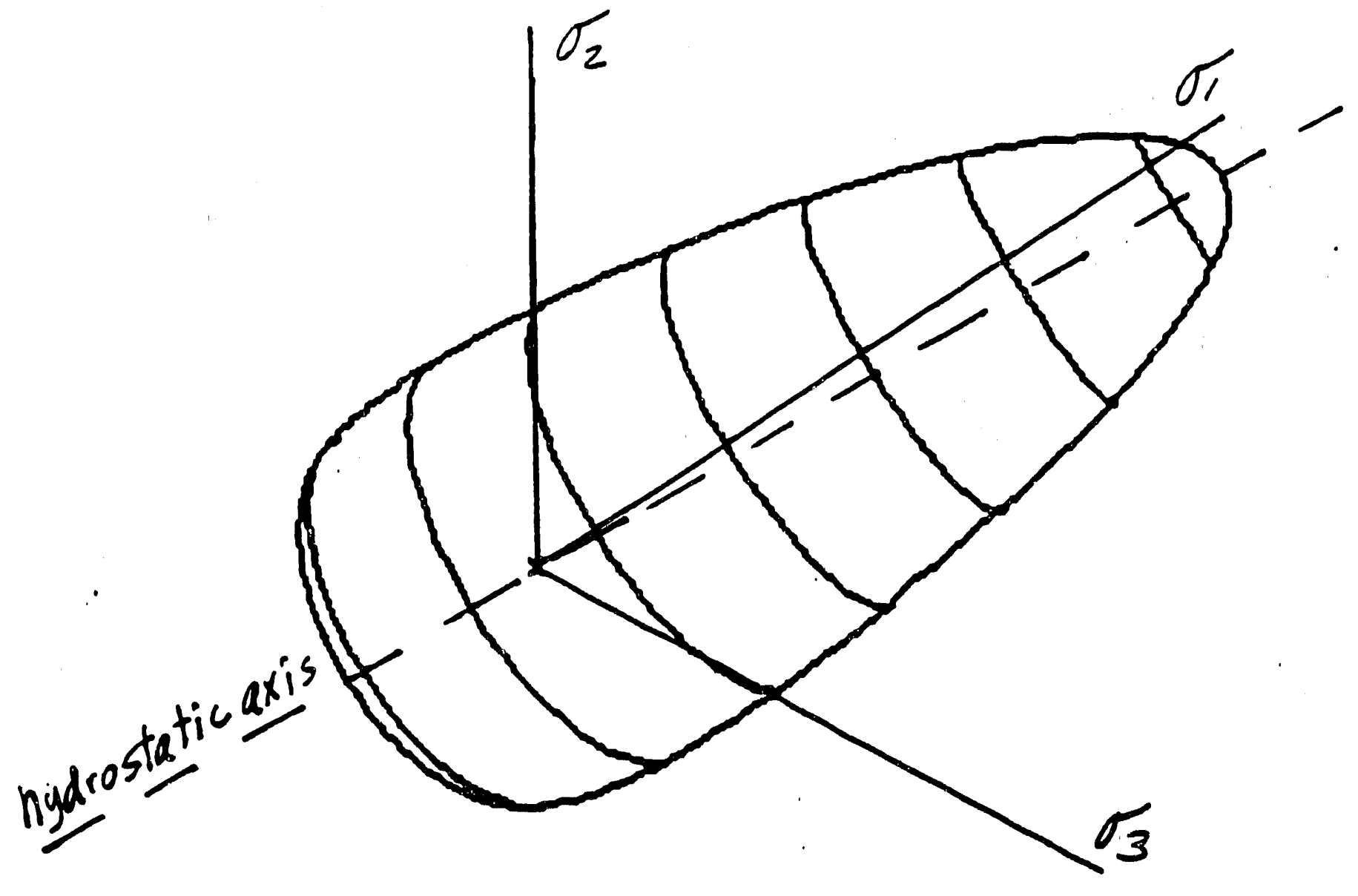

Figure 36. Yield Surface 4 in Haigh-Westergaard Space with $p=3$, $\sigma_{1}=-1, k=0.4837, n=0.2$. 
YIELD SURFACE F5

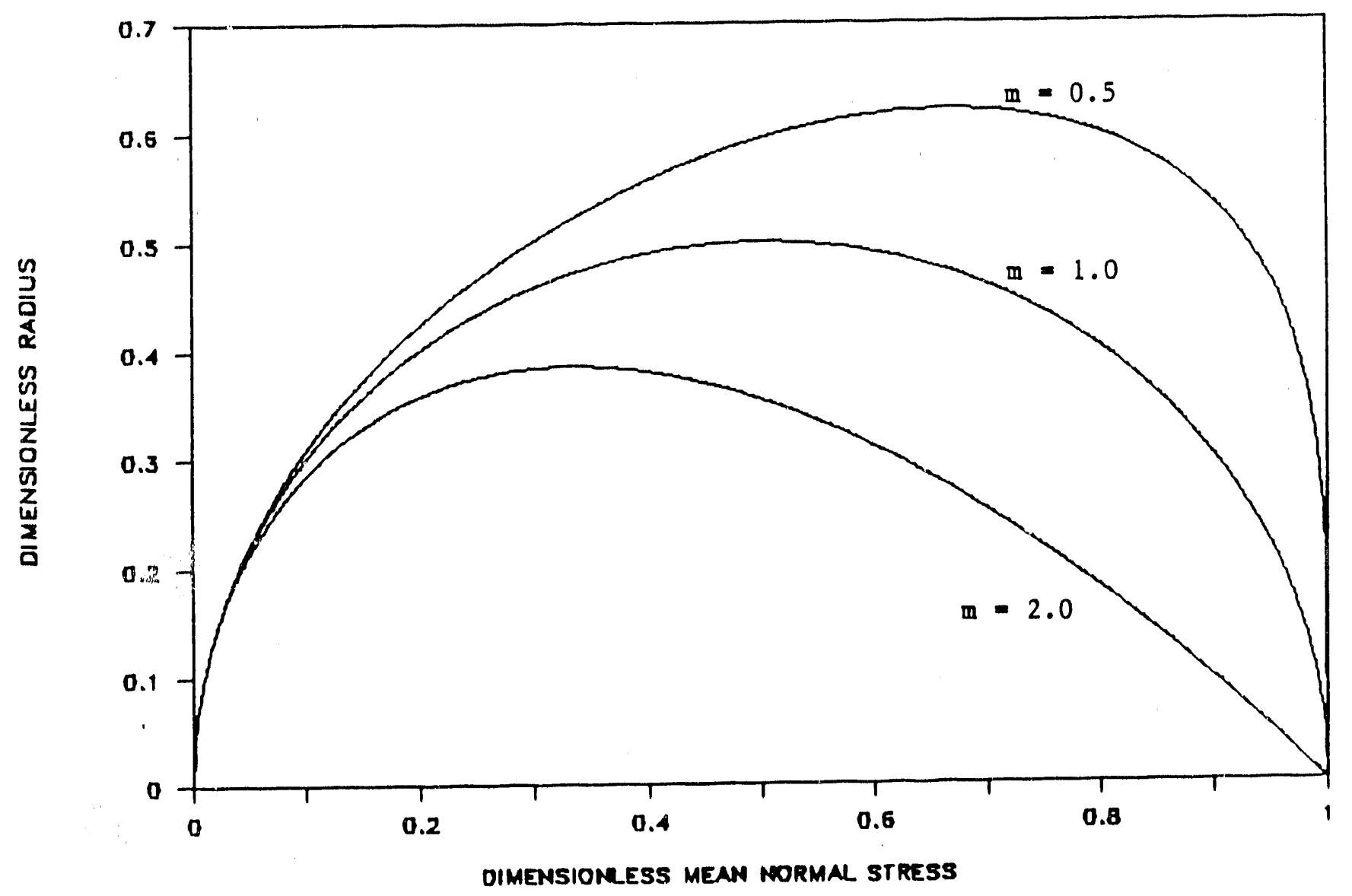

Figure 37. Dimensionless Radius of Yield Surface 5 for $m=0.5$, $1.0,2.0$. 


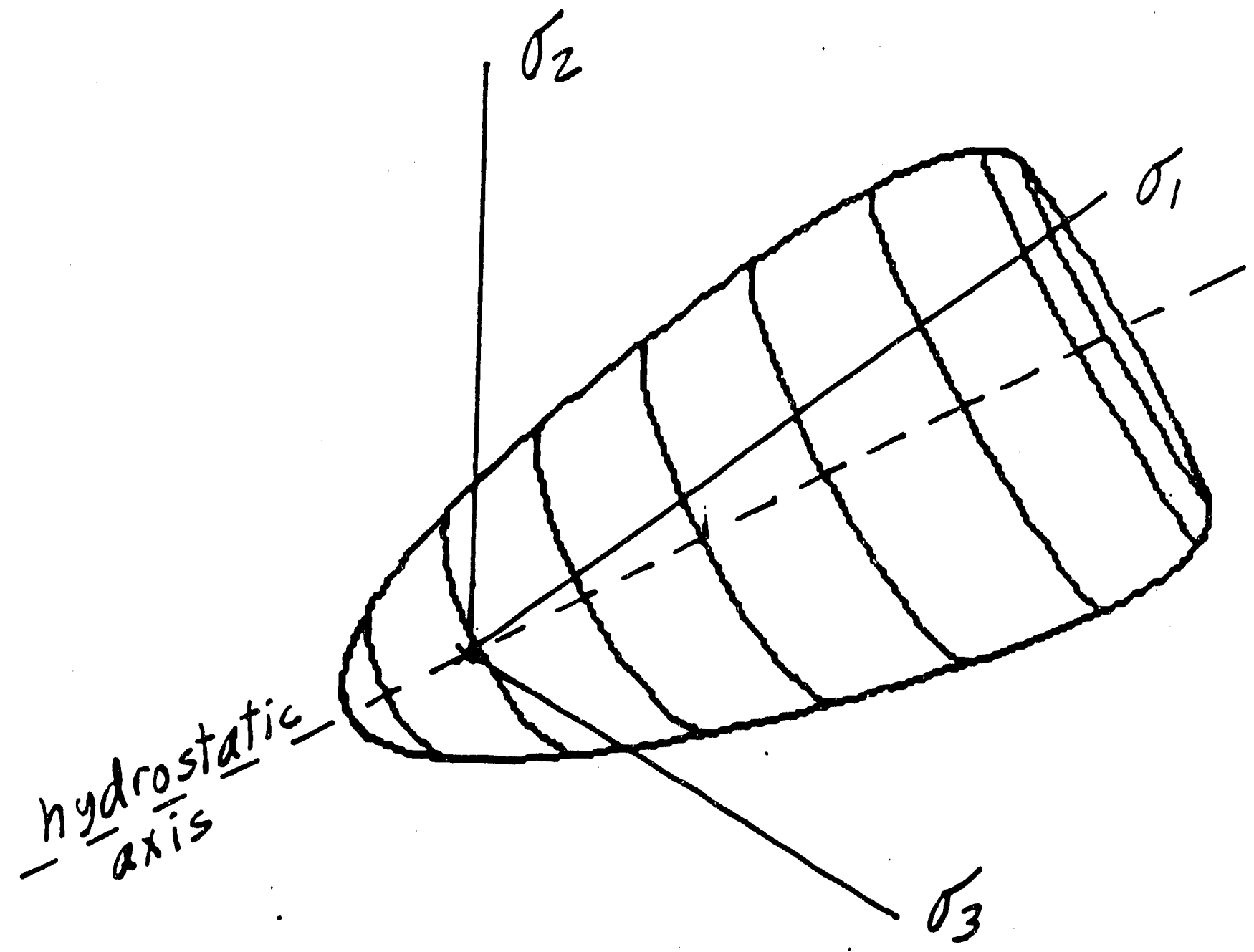

Figure 38. Yield Surface 5 in Haigh-Westergaard Space with $p=3$, $\sigma_{1}=-1, k=0.4837, m=0.2$. 


\section{APPENDIX 1. GENERALIZATION OF THE GRAY-STILES YIELD SURFACE}

Gray and stiles have proposed a model for the frictional flow of granular material which is based on the theory of plasticity (1988). This model assumes that the material behaves in a rigidplastic manner. For states of stress which are small (in a sense which will soon be clarified) there is no motion. For states of larger stress, the material flows such that the ratios of the deformation rates are proportional to the ratios of the stresses. The constitutive law for such a material consists of two related parts: the yield condition, which defines the states of stress which cause motion, and the flow rule, which relates the stresses and the deformation rates.

The state of stress at any point is defined by the six independent components of the stress tensor $\sigma_{i j}$. It is well known, however, that there is always some choice of axes for which the stress tensor is diagonalized. In such principal coordinates the state of stress is fully defined by the three principal (normal) stresses $\sigma_{i}=\left(\sigma_{1}, \sigma_{2}, \sigma_{3}\right)$. This allows the depiction of all possible states of stress in a three dimensional space whose cartesian coordinates represent the three principal stresses. This principal stress space, often termed Haigh-Westergaard space, has many useful properties.

The origin represents a stress-free state. The straight line passing through the origin and $(1,1,1)$ is the locus of states for which the three principal stresses are equal. For this reason it is called the hydrostatic axis. The set of states which have the same value of mean normal stress $\left(\langle\sigma\rangle=\left(\sigma_{1}+\sigma_{2}+\sigma_{3}\right) / 3\right)$ forms a plane normal to the hydrostatic axis. The distance of such a plane from the origin is proportional to the magnitude of the mean normal stress, and the perpendicular distance of any point from the hydrostatic axis is proportional to the magnitude of the deviatoric stress $s_{i}=\sigma_{i}-\langle\sigma\rangle$. For granular materials, most of states of interest are purely compressive and lie in the octant of positive principal stresses. Figure 32 illustrates these features of the Haigh-Westergaard space.

The yield condition $F\left(\sigma_{i j}\right)=0$ for a plastic material becomes a yield surface when represented in Haigh-Westergaard space $F\left(\sigma_{1}\right)=$ 0 . For a rigid-plastic material, states within the yield surface correspond to rigid behavior and those on the surface to plastic flow. States outside the yield surface are inaccessible, except as the yield surface may expand or shrink due to phenomena such as work hardening in metals or density change in granular materials. Because the shape and size of the yield surface depend on the properties of the material, assumptions such as isotropy correspond to restrictions on the allowable shape.

The other part of the constitutive relation is the flow rule. As 
in most theories of plasticity, the authors have adopted the associated flow rule, which requires that the deformation rate tensor $D_{i j}$ be proportional to the stress gradient of the yield condition

$$
D_{i j}=q \frac{\partial F}{\partial \sigma_{i j}}
$$

where $q$ is an undetermined positive scalar. Because $D_{i j}$ is a tensor, there is always a set of principal axes in which only normal deformation rates are nonzero. Hence one can superimpose principal deformation rate axes on the principal stress axes of Haigh-Westergaard space. The associated flow rule guarantees that these principal deformation rates $\left(D_{1}=\left(D_{1}, D_{2}, D_{3}\right)\right)$ are aligned with the principal stresses. The associated flow rule in principal coordinates becomes

$$
D_{i}=q \frac{\partial F}{\partial \sigma_{i}} \text { or } \underline{D}=q \underline{\nabla F}
$$

Thus the principal deformation rate vector is always normal to the yield surface at the point corresponding the tip of the principal stress vector.

This normality condition breaks down at corners or pointed vertices where the gradient of $F$ is undefined. Drucker (1951) asserts that at such locations, the direction of the deformation rate vector lies within the space formed by the limiting outward normals. of course this indeterminacy does not arise for smooth yield surfaces.

Although the yield condition and the flow rule may appear to be independent, they are related. Bland (1957) proved that the associated flow rule is equivalent to two hypotheses concerning plastic work which were introduced by Drucker (1950). The first hypothesis requires that whenever plastio flow is induced by an external agency, the incremental dissipation rate $\delta \mathrm{w}$ must be nonnegative

$$
\delta W=d \sigma_{i} D_{i} \geq 0
$$

where $d \sigma_{i}$ is the incremental stress.

Noting that $D_{i}$ is an outward normal to the yield surface, Drucker proved that the yield surface must be convex in order to guarantee $\delta W \geq 0$ for arbitrary $d \sigma_{i}$. It is interesting to note that the requirement of convexity, which stems from the associated flow 
rule, is distinct from the requirement of a positive dissipation rate in frictional flow

$$
W=\sigma_{i} D_{i}>0
$$

which follows from the laws of thermodynamics. The latter condition implies that the angle between the stress vector $\sigma_{i}$ and the yield surface normal be acute. A yield surface with this property will be called dissipative. Figure 33 clarifies the distinction between a convex yield surface and a dissipative one.

Granular materials do not experience work hardening. This implies that in principle they can be unloaded so that they return to the zero stress state. Thus the origin must lie on or within the yield surface. On the other hand, granular materials become more resistant to yielding as their density increases. This implies that rather than one yield surface, there is actually a family of yield surfaces, each corresponding to a particular density, with those of lower density being concained by those of higher density. of course there is some minimum density necessary to insure intergranular contact and some maximum density above which the grains are crushed. Thus the yield surface family of a compressible granular material must form a hollow laminated shell, whose finite thickness may vary.

The yield surface of a material which is isotropic must be unchanged if the principal stress axes are relabeled. This requires that the yield surface be invariant to $120^{\circ}$ rotations about: the hydrostatic axis. Therefore the allowable cross sections normal to the hydrostatic axis include circles, equilateral triangles, hexagons, and other shapes having three-fold rotational symmetry. A yield surface with this property will be termed symmetric in the ensuing d-scussion. In fact all of the yield surfaces considered below have circular cross sections.

It has been observed that a loosely packed granular material consolidates as it begins to flow, whereas a densely packed material dilatates. In either case, the material eventually flows at constant density (critical state) if conditions remain constant. The change in density is indicated by the projection of the principal deformation rate vector on the hydrostatic axis. A positive consolidation rate corresponds to a positive projection on the hydrostatic axis. When the material has reached the critical state, the principal deformation rate vector must be perpendicular to the hydrostatic axis. Behavior of this sort is assured by the normality condition and the convexity of the yield surface. The critical states are represented by the largest cross section of the yield surface. 
In order to allow flow in both pure tension and pure compression, each yield surface must have both a lower and an upper apex. For a non-cohesive material, the lower apex coincides with the stressfree state at the origin. In a cohesive material, the lower apex is at $\left(\sigma_{1}, \sigma_{1}, \sigma_{1}\right)$ where $\sigma_{1}<0$. In general tensile strength $\left|\sigma_{1}\right|$ may increase with density. For many materials, $\left|\sigma_{a}\right|$ is so small that it is difficult to measure and has no practical importance. Nevertheless, cohesion should be permitted in theoretical formulations.

Observations of flow in the critical state often follow coulomb's law of friction: the maximum shear stress is linearly proportional to the maximum compressive stress. The three dimensional generalization of this result is that the locus of maximum yield surface cross sections is a cone whose apex is at $\left(\sigma_{a}, \sigma_{a}, \sigma_{1}\right)$. Rigid-plastic materials having such yield surface families are called Coulomb powders (Brown and Richards, 1970). Yield surface families exhibiting this behavior will be called coulombic.

To summarize the preceding discussion, the yield surface family of a compressible, frictional, rigid-plastic granular material which obeys the associated flow rule must be:
1. closed
2. convex
3. nested
4. symmetric
5. dissipative

For many materials the yield surface family is also coulombic, or nearly so. These conditions are necessary, but not sufficient, to guarantee that a yield surface family accurately represents the behavior of any particular material. These considerations will now be applied to some previously known yield surface families and to motivate some new generalizations.

The von Mises yield condition, which is widely used in metal plasticity, is

$$
F_{1}=-J_{2 D}-k^{2}=0
$$

where $J_{2 D}=$ second invariant of the deviatoric stress tensor

$$
k=\frac{\sqrt{2}}{3} \times \text { maximum elastic shear stress. }
$$

Figure 2 shows that the von Mises yield condition forms a circular cylinder in Haigh-Westergaard space. The radius $R_{1}$ is given by 


$$
R_{1}=\sqrt{-2 J_{2 D}}=\sqrt{2} k
$$

It is tempting to try to account for density effects by allowing $k$ to depend on density, but this would be inconsistent. The density of a von Mises material cannot vary because the deformation rate vector is always perpendicular to the hydrostatic axis. It is clear from the geometry that this yield surface is convex, symmetric, and dissipative; but is neither closed, nested, nor coulombic. Therefore it is not adequate to describe frictional granular flow.

The extended von Mises or Drucker-Prager yield condition for cohesive material is

$$
F_{2}=-J_{2 D}-k^{2}\left(\langle\sigma\rangle-\sigma_{A}\right)^{2}=0
$$

The radius of this surface is given by

$$
R_{2}=\sqrt{2} k\left(\langle\sigma\rangle-\sigma_{1}\right)
$$

The yield surface forms a cone whose apex is at $\left(\sigma_{1}, \sigma_{1}, \sigma_{1}\right)$. According to the associated flow rule, the deformation rate vector always has a negative projection on the hydrostatic axis, indicating that the material must dilatate continuously during plastic flow. Since the material weakens as its density decreases, the cone collapses toward the hydrostatic axis until the grains cease to maintain contact. The extended von Mises yield surface family is convex, nested, and symmetric; but is not closed. Since it has no critical state, it cannot be coulombic.

If the material is cohesionless, the apex of the cones is at the origin and the stress vectors are generators of the cones. Such a cohesionless material would not be dissipative. If the material is cohesive, however, this difficulty does not arise. Nevertheless, the extended von Mises yield condition is obviously not suitable for granular materials.

The Gray-stiles (1988) yield condition, extended for a cohesive material, is

$$
F_{3}=-J_{2 D}+k^{2}\left[\left(\langle\sigma\rangle-\sigma_{1}-p\right)^{2}-p^{2}\right]=0
$$


where

$$
\begin{aligned}
\mathrm{k} & =\text { material constant } \\
\mathrm{p} & =\text { mean normal stress at critical state } \\
& =\mathrm{c} \rho^{1 / \beta}
\end{aligned}
$$$$
\text { c, } \beta=\text { material constants. }
$$

This family of closed, cigar-shaped yield surfaces is depicted in Figure 34. Each surface has a circular cross section whose radius is

$$
R_{3}=\sqrt{2} k\left[p^{2}-\left(\langle\sigma\rangle-\sigma_{1}-p\right)^{2}\right]^{1 / 2}
$$

This expression demonstrates the properties of nestedness and symmetry. The lower apex is at $\langle\sigma\rangle=\sigma$, , the upper is at $\langle\sigma\rangle=2 p+\sigma_{a}$, and the major axis has a length of $2 \mathrm{p}$. The maximum radius given by

$$
R_{3_{m}}=R_{3}\left(\langle\sigma\rangle=p+\sigma_{n}\right)=\sqrt{2} k p=\sqrt{2} k\left(\langle\sigma\rangle-\sigma_{1}\right)
$$

which shows the coulombic property.

By application of the associated flow rule, the consolidation rate is found to be

$$
D_{i i}=2 q k^{2}\left(\langle\sigma\rangle-\sigma_{1}-p\right)
$$

This is of iously negative for $\langle\sigma\rangle\left\langle p+\sigma_{a}\right.$, positive for $\left.\langle\sigma\rangle\right\rangle p$ $+\sigma_{A}$, and zero at the critical state.

The dissipation rate

$$
w=2 q k^{2}\left[\langle\sigma\rangle p+\sigma_{1}\left(\langle\sigma\rangle-\sigma_{1}-2 p\right)\right]
$$

is positive for $\langle\sigma\rangle\rangle \sigma_{a}$.

Convexity has been verified by demonstrating that

$$
\frac{\partial^{2} R_{3}}{\partial\langle\sigma\rangle^{2}} \leq 0
$$


Therefore the Gray-stiles relation for cohesive materials possesses all the properties required of an acceptable yield condition. Yet it has a very special shape which may limit its applicability to real materials. In particular, it is symmetric about the plane of the critical state cross section.

A generalization which breaks this symmetry is

$$
F_{4}=-J_{2 D}+k^{2}\left(\langle\sigma\rangle-\sigma_{A}\right)^{a}\left(\langle\sigma\rangle-\sigma_{A}-2 p\right)=0
$$

where $0<n \leq 2$. This reduces to the Gray-stiles result for $n=1$. The radius is given by

$$
R_{4}=\sqrt{2} k\left[\left(\langle\sigma\rangle-\sigma_{1}\right)^{n}\left(2 p+\sigma_{1}-\langle\sigma\rangle\right)\right]^{1 / 2}
$$

The nestedness and symmetry of this family are immediately evident. Each surface is closed with the lower apex at $\langle\sigma\rangle=\sigma_{A}$, the upper at $\langle\sigma\rangle=2 \mathrm{p}+\sigma_{1}$, and the major axis having a length of $2 \mathrm{p}$. The maximum radius occurs for

$$
<\sigma>=\left(\frac{n}{n+1}\right) 2 p+\sigma
$$

For $n<1$, the critical state is nearer the lower apex, but for $n$ $>1$ the opposite true. The largest critical mean normal stress is

$$
\langle\sigma\rangle=(4 / 3) p+\sigma_{\text {. }}
$$

when $\mathrm{n}=2$. Several cases are illustrated in Figure 35 . The critical state radius is

$$
R_{4}=\sqrt{2} K\left[\frac{n^{n}(2 p)^{n+1}}{(n+1)^{a+1}}\right]^{1 / 2}
$$

Although the radius always increases with the mean normal stress, linear proportionality is obtained only for $n=1$. Otherwise there is a deviation from the coulombic property. This behavior will be called quasi-coulombic. The property of convexity has been assured by dexonstrating that 


$$
\frac{\partial^{2} R_{4}}{\partial\langle\sigma\rangle^{2}} \leq 0
$$

Application of the associated flow rule shows that

$$
D_{i}=q\left(\sigma_{i}-\langle\sigma\rangle\right)+\frac{q k^{2}}{3}(n+1)\left(\langle\sigma\rangle-\sigma_{1}\right)^{n}-\frac{2}{3} q k^{2} n p\left(\langle\sigma\rangle-\sigma_{1}\right)^{n-1}(96)
$$

The rate of consolidation is

$$
D_{i j}=q k^{2}\left(\langle\sigma\rangle-\sigma_{A}\right)^{n-1}\left[(n+1)\left(\langle\sigma\rangle-\sigma_{A}\right)-2 n p\right]
$$

As expected, this is positive between the critical state and the upper apex. The rate of dissipation is given by

$$
W=q\left\{-2 J_{2 D}+k^{2}\langle\sigma\rangle\left(\langle\sigma\rangle-\sigma_{A}\right)^{n-1}\left[(n+1)\left(\langle\sigma\rangle-\sigma_{A}\right)-2 n p\right]\right\}
$$

It can be demonstrated that $\mathrm{W}>0$ when $\sigma_{\mathrm{a}}=0$. For cohesive materials, the origin lies within the yield surface. It is evident from the geometry that whenever the origin is contained within a convex yield surface, the angle between $\sigma_{i}$ and $D_{i}$ is always acute. Therefore the yield surface family $F_{4}=0$ is closed, convex, nested, symmetric, and dissipative, but is only quasi-coulombic. Figure 36 shows a yield surface of this type.

If it is necessary to consider yield surfaces for which the critical state lies closer to the upper apex, the following function may be used

$$
F_{5}=-J_{2 D}-k^{2}\left(\langle\sigma\rangle-\sigma_{a}\right)\left(2 p+\sigma_{1}-\langle\sigma\rangle\right)^{m}=0
$$

where $0<m \leq 2$. Once again the Gray-Stiles result is recovered for $m=1$. The equation for the radius is

$$
R_{S}=\left[2 k^{2}\left(\langle\sigma\rangle-\sigma_{a}\right)\left(2 p+\sigma_{a}-\langle\sigma\rangle\right)^{m}\right]^{1 / 2}
$$

The properties of nestedness and symmetry are evident. Each yield surface is closed, with apexes at $\langle\sigma\rangle=\sigma_{1}$ and $2 \mathrm{p}+\sigma_{\mathrm{A}}$, and a major axis length of $2 \mathrm{p}$. The mean normal stress which produces the maximum radius is 


$$
<\sigma>=\left(\frac{1}{1+m}\right) 2 p+\sigma
$$

The critical state radius is

$$
R_{g_{m}}=\sqrt{2} k\left[\frac{1}{m}\left(\frac{m 2 p}{m+1}\right)^{1+m}\right]^{1 / 2}
$$

The critical state lies between $\langle\sigma\rangle=\frac{2}{3} p+\sigma_{1}(m=2)$ and the upper apex $(m=0)$, as illustrated by Figure 37 . This yield surface family is also quasi-Coulombic except when $\mathrm{m}=1$.

The convexity of these surfaces can be demonstrated by differentiation. According to the associated flow rule the deformation rate is

$$
\begin{aligned}
D_{i}=q\left(\sigma_{i}-\langle\sigma\rangle\right)+ & \frac{m q k^{2}}{3}\left(\langle\sigma\rangle-\sigma_{1}\right)\left(2 p+\sigma_{1}-\langle\sigma\rangle\right)^{m-1} \\
& -\frac{q k^{2}}{3}\left(2 p+\sigma_{1}-\langle\sigma\rangle\right)^{m}
\end{aligned}
$$

and the rate of consolidation is

$$
D_{i i}=q k^{2}\left(2 p+\sigma_{a}-\langle\sigma\rangle\right)^{m}\left[m\left(\langle\sigma\rangle-\sigma_{a}\right)\left(2 p+\sigma_{1}-\langle\sigma\rangle\right)^{-1}-1\right]
$$

It can be seen that the consolidation rate is positive between the critical state and the upper apex. The dissipation rate is

$$
W=q\left\{-2 J_{2 D}+k^{2}\langle\sigma\rangle\left(2 p+\sigma_{1}-\langle\sigma\rangle\right)^{m}\left[m\left(\langle\sigma\rangle-\sigma_{A}\right)\left(2 p+\sigma_{1}-\langle\sigma\rangle\right)^{-1}-1\right]\right\}
$$

For cohesionless materials it is easy to prove that $w>0$. The geometric argument suffices for cohesive materials. Figure 38 shows a member of this family. In summary, the yield surface family $F_{s}=0$ is closed, convex, nested, symmetric, dissipative, and quasi-coulombiz.

The new yield surface families defined by $F_{4}=0$ and $F_{5}=0$ are more general than the Gray-stiles family $\left(F_{3}=0\right)$. They possess all of the features needed for physical realism but they are only quasi- 
Coulombic. Their greater generality is purchased at the cost of introducing one additional constant ( $m$ or $n$ ) which must be determined experimentally. 
APPENDIX 2. GRANULAR FLOW EQUATIONS FOR SPECIAL CASES AND THEIR SOLUTIONS

\author{
by \\ Ismail celik
}

In this appendix the analogy between granular flow and fluid flow equations is investigated. The granular flow equations are simplified for some special cases and analytical solutions are sought. The constitutive equations for the granular flow model are the same as those described in the main body of this report.

\title{
Analogy between Granular Flow and Fluid Flow
}

The general equations of motion in tensor notation, for a continuum are:

Continuity:

$$
\frac{\partial \rho}{\partial t}+\frac{\partial}{\partial x_{j}}\left(\rho u_{j}\right)=0
$$

Momentum :

$$
\rho \frac{\partial u_{i}}{\partial t}+\rho u_{j} \frac{\partial u_{i}}{\partial x_{j}}=\rho g_{i}+\frac{\partial \sigma_{i j}}{\partial x_{j}}
$$

where $\rho=$ density, $u=$ velocity, $g=$ gravitational acceleration, $t$ = time, $x=$ space coordinates, and $\sigma_{i j}$ is the stress tensor. The constitutive relations for granular flow and fluid flow, respectively, are given by

$$
\begin{aligned}
& \sigma_{i j}=\frac{D_{i j}}{q}-\left[\hat{p}-\left(\frac{1}{2 k^{2} q}-\frac{1}{3 q}\right) \underline{\nabla} \cdot \underline{u}\right] \delta_{i j} \\
& \sigma_{i j}=2 \mu D_{i j}-[p-(K+\lambda) \underline{\nabla} \cdot \underline{u}] \delta_{i j}
\end{aligned}
$$

where

$$
D_{i j}=\frac{1}{2}\left(\frac{\partial u_{1}}{\partial x_{1}} \cdot \frac{\partial u_{1}}{\partial x_{1}}\right)
$$

is the rate of deformation tensor, $\hat{p}$ is the pressure for granular flow, $k$ is a parameter related to the angle of internal friction, $q$ is a model scalar function, $p$ is the fluid pressure, $K$ is a constant, $\mu$ is the dynamic fluid viscosity, $\lambda$ is the second coefficient of viscosity, and $\sigma_{i j}$ is the kronecker delta. 
Note that the deformation rate tensor defined in (110) uses the opposite sign converition compared to the rest of this report. Comparison of Eqs. (108) and (109) reveals that these equations have the same features and they become exactly the same with the following equivalence statements:

$$
\mathrm{p}=\hat{\mathrm{p}} ; \quad \mu=1 / 2 \mathrm{q} ; \quad \mathrm{K}=1 / 2 \mathrm{k}^{2} \mathrm{q} ; \quad \lambda=-\frac{1}{3} \mathrm{q}=-\frac{2}{3} \mu
$$

For fluid flow $\lambda=-2 / 3 \mu$ to satisfy a consistency condition for the thermodynamic pressure $p=-1 / 3 \sigma_{i i}=-\langle\sigma\rangle$. This condition is automatically satisfied by the granular flow model and is known as the plasticity condition provided that we define a granular flow viscosity via

$$
\hat{\mu}=1 / 2 q
$$

It should be noted also that for Newtonian fluids $K=0$. The physical consequences of $K=1 / 2 \mathrm{k}^{2} q$ in the present model need to be investigated. The granular flow viscosity $\hat{\mu}$ is a function of the material properties, e.g. $k$, and the plastic dissipation rate, $w$. This relation can be written as

$$
\hat{\mu}=k^{2} \hat{p}\left[\left(1-\frac{2}{3} k^{2}\right)(\nabla \cdot \underline{u})^{2}+2 k^{2} x+k^{2} z\right]^{-1 / 2}
$$

where

$$
\begin{gathered}
X=D_{11}^{2}+D_{22}^{2}+D_{33}^{2}=\left(\frac{\partial u}{\partial x}\right)^{2}+\left(\frac{\partial v}{\partial y}\right)^{2}+\left(\frac{\partial w}{\partial z}\right)^{2} \\
Z=4\left(D_{12} D_{21}+D_{23} D_{32}+D_{31} D_{13}\right)= \\
\left(\frac{\partial u}{\partial y}+\frac{\partial v}{\partial x}\right)^{2}+\left(\frac{\partial u}{\partial z}+\frac{\partial w}{\partial x}\right)^{2}+\left(\frac{\partial v}{\partial z}+\frac{\partial w}{\partial y}\right)^{2}
\end{gathered}
$$

In deriving Eq. (112) the following identity was used

$$
Y=D_{11} D_{22}+D_{22} D_{33}+D_{33} D_{11}=\frac{1}{2}\left[(\nabla \cdot \underline{u})^{2}-x\right]
$$

Also, a new notation is introduced, for brevity, where $u, v$, and $w$ denote the velucity components in the $x-, y-$, and $z-$ directions, respectively.

To see the relation between $\hat{\mu}$ and the plastic dissipation rate, $W$, one can use the relation 
to yield

$$
\begin{aligned}
& W=D_{i j} \sigma_{i j}=\sigma_{11} D_{11}+\sigma_{22} D_{22}+\sigma_{33} D_{33} \\
& =2 q k^{2} \hat{p}\langle\sigma\rangle=2 q k^{2} \hat{p}\left[\hat{p}-\frac{\underline{\nabla} \cdot \underline{\underline{u}}}{2 k^{2} q}\right]
\end{aligned}
$$

$$
\hat{\mu}=\frac{k^{2} \hat{p}}{\hat{W}+\hat{p} \underline{\nabla} \cdot \underline{\underline{u}}}
$$

\section{Moderately Compressible, Two-Dimensional Flow}

If we assume, in addition to two-dimensionaiity, that the dilatation terms, $i . e$. terms containing $\underline{\nabla} \cdot \underline{u}$ are negligible in order of magnitude compared to the other dominating terms, the equations of motion can be reduced to a form which is amenable to fairly simple numerical treatment. This assumption, $i . e ., \underline{\nabla} \cdot \underline{u}=0$, is usually involved for slightly compressible fluid flows where the Mach number is less than about 0.3 . Invoking these assumptions leads to the following set of equations for $2 D$ granular flow.

$$
\begin{gathered}
\frac{\partial \rho}{\partial t}+\frac{\partial}{\partial x}(\rho u)+\frac{\partial}{\partial y}(\rho v)=0 \\
\rho\left(\frac{\partial u}{\partial t}=u \frac{\partial u}{\partial x}+v \frac{\partial u}{\partial y}\right)=-\frac{\partial \hat{p}}{\partial x}+\rho g_{x}+\frac{\partial}{\partial x}\left(\hat{\mu} \frac{\partial u}{\partial x}\right) \\
+\frac{\partial}{\partial y}\left(\hat{\mu} \frac{\partial u}{\partial y}\right)+\frac{\partial}{\partial x}\left(\hat{\mu} \frac{\partial u}{\partial x}\right)+\frac{\partial}{\partial y}\left(\hat{\mu} \frac{\partial v}{\partial x}\right) \\
\rho\left(\frac{\partial v}{\partial t}+u \frac{\partial v}{\partial x}+v \frac{\partial v}{\partial y}\right)=-\frac{\partial \hat{p}}{\partial y}+\rho g_{y}+\frac{\partial}{\partial x}\left(\hat{\mu} \frac{\partial v}{\partial x}\right) \\
+\frac{\partial}{\partial y}\left(\hat{\mu} \frac{\partial v}{\partial y}\right) \frac{\partial}{\partial x}\left(\hat{\mu} \frac{\partial u}{\partial y}\right)+\frac{\partial}{\partial y}\left(\hat{\mu} \frac{\partial v}{\partial y}\right)^{-1 / 2} \\
\hat{\mu}=k \hat{p}\left[2\left(\frac{\partial u}{\partial x}\right)^{2}+2\left(\frac{\partial v}{\partial y}\right)^{2}+\left(\frac{\partial u}{\partial y}+\frac{\partial v}{\partial x}\right)^{2}\right]^{-12}
\end{gathered}
$$


An additional equation of state is necessary to relate $\hat{\rho}$ to the pressure $\hat{p}$ and is given by

$$
\hat{\mathrm{p}} / \hat{\mathrm{p}}_{0}=\left(\rho / \rho_{0}\right)^{1 / \beta}
$$

where $\beta=$ constant, $\hat{p}_{0} \hat{\rho}_{\text {o }}$ are some reference values for $\hat{p}$ and $\hat{\rho}$, respectively.

Equations (118) through (122) form a closed set for the unknowns $u$, $v, \hat{p}$ and $\hat{\rho}$.

\section{Steady, Parallel Fully Developed Flow}

Here we assume that a flow exists such that $v=0, u=u(y)$ (or $\frac{\partial u}{\partial x}=0$ ), then Eqs. (118) through (122) reduce to

$$
\begin{gathered}
\frac{\partial}{\partial x}(\rho u)=0 \\
0=-\frac{\partial \hat{p}}{\partial x}+\rho g_{x}+\frac{\partial}{\partial y}\left(\hat{\mu} \frac{\partial u}{\partial y}\right) \\
0=-\frac{\partial \hat{p}}{\partial y}+\rho g_{y} \\
\hat{\mu}=k \hat{p} /\left|\frac{\partial u}{\partial y}\right|
\end{gathered}
$$

Two special cases are of interest.

(i) $g_{x}=g_{y}=0$, no gravity, then $\hat{p}=$ constant

This means there is no shear that can be imposed on the flow through the surface.

(ii) $\rho g_{y}=0$ hence $\hat{p}=\hat{p}(x)$ only, then

$$
\frac{\partial \hat{p}}{\partial x}=\rho g ; \quad \hat{p}=p_{0}+\int \rho g d x=p_{0}+\rho g x
$$


Note that from (123) $\rho=\rho(y)$ only. This case represents a vertical flow and indicates that the pressure force is balanced only by gravity, again indicating no shear force.

For the above two cases the velocity profile can be determined from Eq. (126) if $\hat{\mu}$ is known from somewhere else, say from experiments.

Consider the case of two parallel plates with the top plate moving steadily with a velocity $U_{0}$, and assume a linear profile for $u, i . e$.

$$
u=U_{0} \frac{y}{h}
$$

Eq (126) yields

$$
\hat{\mu}=h k \hat{p} / U_{0}=\text { constant }
$$

other profile assumptions can be investigated in a similar manner. Flow over a suddenly Accelerated Infinite Plate

Consider the two-dimensional, transient problem of granular flow over an horizontal, infinite flat plate. The plate is suddenly accelerated to a constant velocity $U_{0}$. The boundary and initial conditions are:

$$
\begin{aligned}
& v=0, u=0 \text { at } y=\infty \quad t>0 \\
& v=0, u=U_{0} \text { at } y=0 \quad t>0
\end{aligned}
$$

Due to the geometry of the problem

$$
\frac{\partial u}{\partial x}=\frac{\partial v}{\partial x}=0
$$

For this case Eqs. (118) through (122) reduce to

$$
\begin{gathered}
\frac{\partial \rho}{\partial t}+\frac{\partial}{\partial x}(\rho u)+\frac{\partial}{\partial y}(\rho v)=0 \\
\rho\left(\frac{\partial u}{\partial t}+v \frac{\partial u}{\partial y}\right)=-\frac{\partial \hat{p}}{\partial x}+\frac{\partial}{\partial y}\left(\hat{\mu} \frac{\partial u}{\partial y}\right)
\end{gathered}
$$




$$
\begin{gathered}
\rho\left(\frac{\partial v}{\partial t}+v \frac{\partial v}{\partial y}\right)=-\frac{\partial \hat{p}}{\partial y}+\rho g+\frac{\partial}{\partial y}\left(2 \hat{\mu} \frac{\partial v}{\partial y}\right) \\
\hat{\mu}=k \hat{p}\left[2\left(\frac{\partial v}{\partial y}\right)^{2}+\left(\frac{\partial u}{\partial y}\right)^{\partial}\right]^{-1 / 2} \\
\hat{p} / \hat{p}_{0}=\left(\rho / \rho_{0}\right)^{1 / \beta}
\end{gathered}
$$

These equations are the working equations with the least number of assumptions for the compressible flow case. In order to simplify the problem further let us assume that $\hat{\rho}=$ constant, hence $v=0$ which leads to

$$
\begin{gathered}
\rho \frac{\partial u}{\partial t}=-\frac{\partial \hat{p}}{\partial x}+\frac{\partial}{\partial y}\left(\hat{\mu} \frac{\partial u}{\partial y}\right) \\
0=-\frac{\partial \hat{p}}{\partial y}+\rho g
\end{gathered}
$$

Eq. (122) shows that $\frac{\hat{d}}{d x}=$ constant. Let us take $\frac{\hat{d}}{d x}=0$ for brevity. The granular flow viscosity $\hat{\mu}$ is given by

$$
\hat{\mu}=k \hat{p} /\left|\frac{\partial u}{\partial y}\right|
$$

Solving for $\hat{p}$ from (133) and substituting it into (132) with (134) yields

$$
\rho \frac{\partial u}{\partial t}=k \rho g
$$

The solution is

$$
\mathrm{u}=\mathrm{kgt}
$$

which tends to infinity as $t$ increases and so is not an acceptable solution. 
APPENDIX 3. MODIFIED VERSION OF TEACH FOR THE VON MISES SIMULATIONS

Note: All modifications are shown in lower case letters. 
PROGRAM CONTRO

C

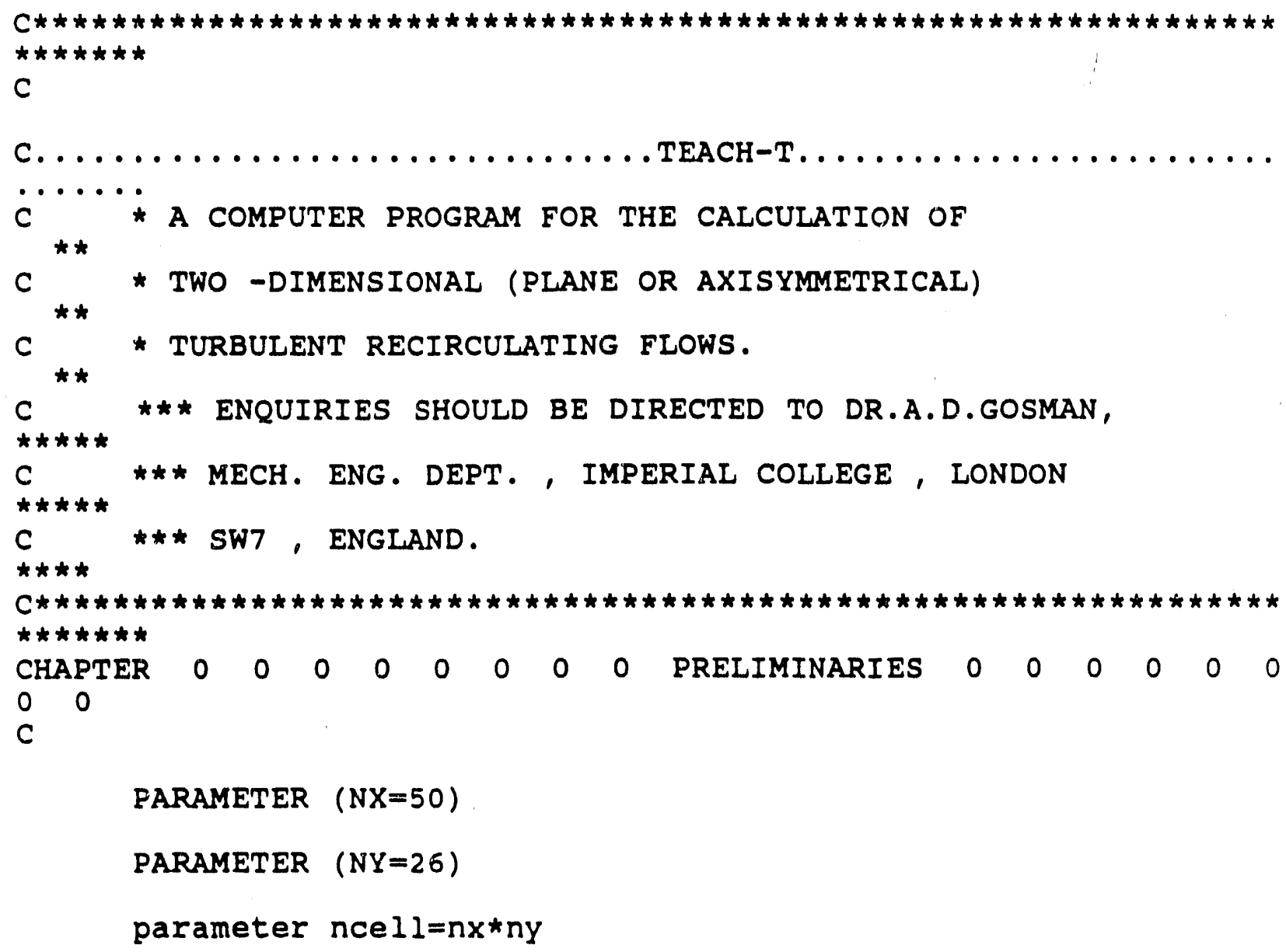


$1 / V A R / U(N X, N Y), V(N X, N Y), P(N X, N Y), P P(N X, N Y), T E(N X, N Y), E D(N X, N Y)$

* $\quad 1 / A L L / I T, J T, N I, N J, N I M I, N J M 1$, GREAT

1/all/it,jt,ni,nj,niml,njm1,great, small

$1 /$ GEOM/X(NX), Y (NY), DXEP(NX), DXPW (NX), DYNP (NY), DYPS (NY) ,

$S N S(N Y), S E W(N X), X U(N X), Y V(N Y), R(N Y), R V(N Y), R C V(N Y), \stackrel{2}{2}$ NDCOS

$1 /$ IPOL/FXP(NX), FXM(NX), FYP(NY), FYM(NY)

1/FLUPR/URFVIS, VISCOS, DENSIT, PRANDT , DEN (NX, NY) , VIS (NX, NY)

1/KASE T1/UIN, TEIN, EDIN, FLOWIN, ALAMDA,

RSMALL, RLARGE, AL1, AL2, JSTEP, ISTEP, JSTP1, JSTM1, ISTP1, ISTM1

$1 /$ TURB/GEN (NX, NY) , CD , CMU, C1, C2 , CAPPA, ELOG, PRED, PRTE, PRM

1 /WALLF/YPLUSN (NX) , XPLUSW(NY) , TAUN (NX) , TAUW (NY)

$1 / C O E F / A P(N X, N Y), A N(N X, N Y), A S(N X, N Y), A E(N X, N Y), A W(N X, N Y), S U(N X, N Y)$, 1 $\mathrm{SP}(\mathrm{NX}, \mathrm{NY})$

INCALU, INCALV, INCALP, INPRO, INCALK, INCALD, INCALM, INCALA,

1 INCALB

GREAT $=1 . E 30$

$\operatorname{small}=1.0 e-06$

ISTEP $=2$

JSTEP $=25$

CM JSTEP $=8$

$\mathrm{N}$ ITER $=0$

$\mathrm{IT}=\mathrm{NX}$

$\mathrm{JT}=\mathrm{NY}$

NSWPU $=3$

NSWPV $=3$

NSWPP $=5$ 


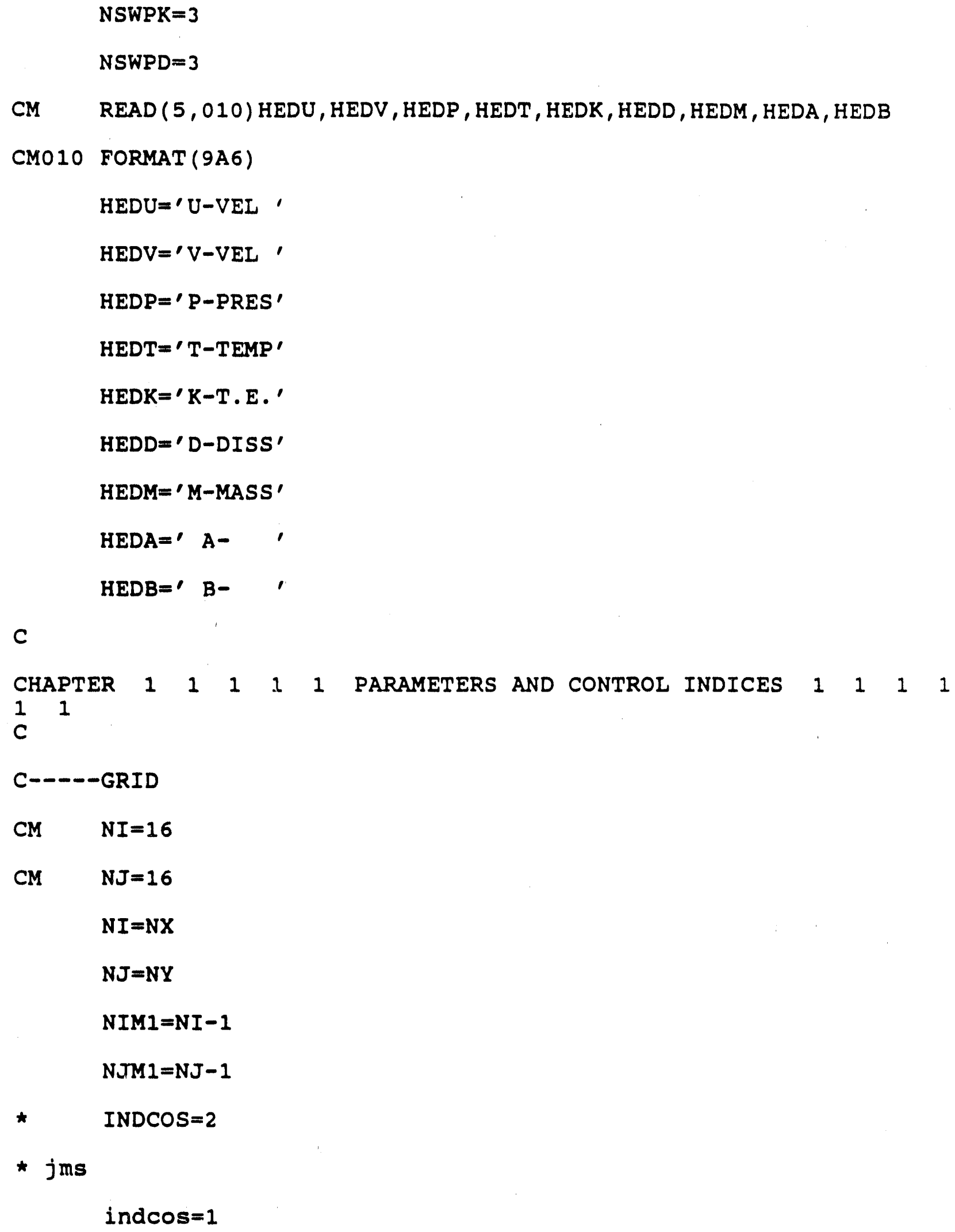


* jms

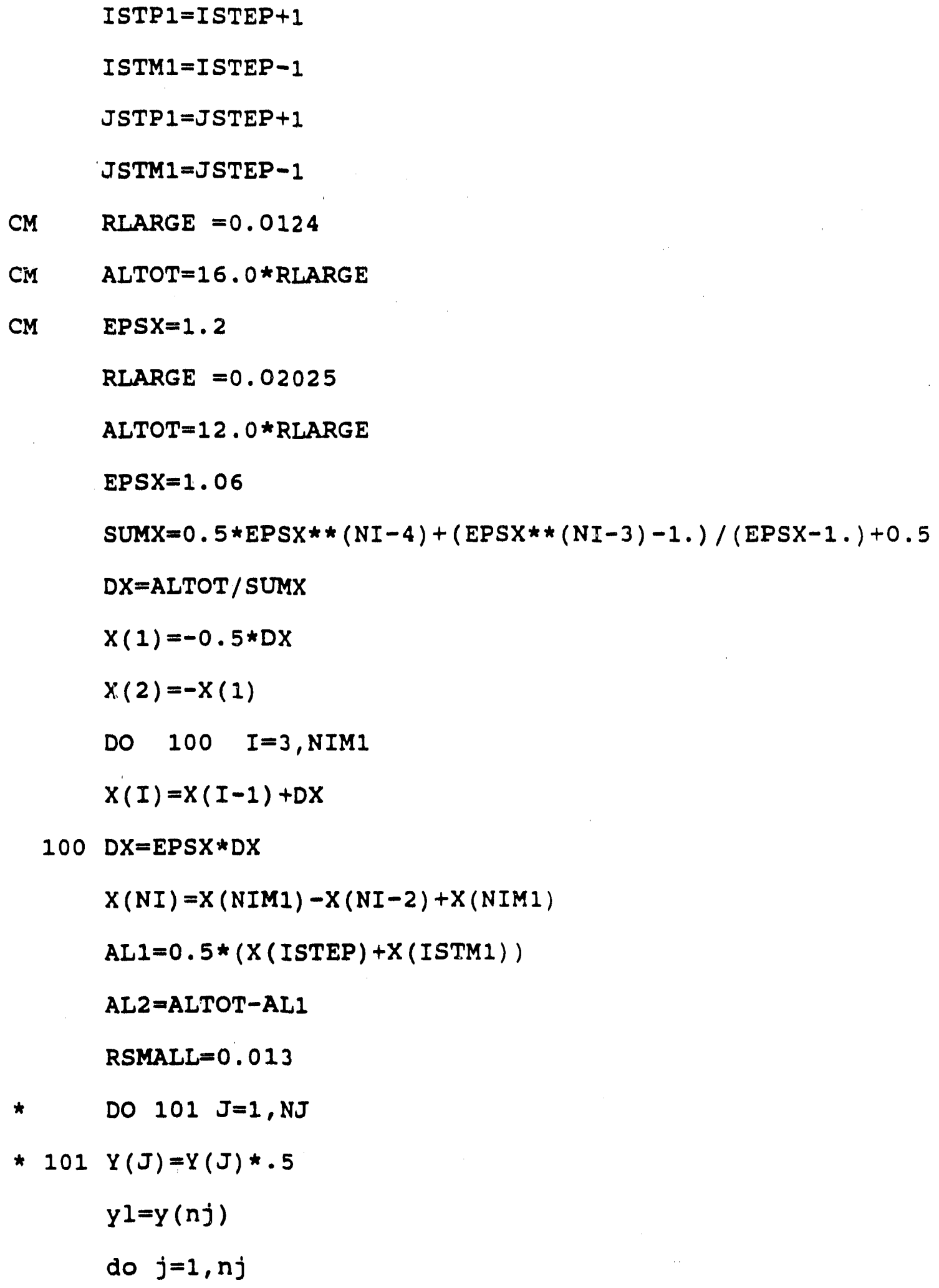




$$
\begin{aligned}
& y(j)=y(j) * 0.02 / y l \\
& \text { end do } \\
& y l=y(n j) \\
& r n=f l o a t(n i-2)+0.5 \\
& x(1)=-0.5 * y l * 100 . / \mathrm{rn} \\
& x(2)=-x(1) \\
& \text { do } i=3, n i \\
& x(i)=x(i-1)+y l * 100 . / \mathrm{rn}
\end{aligned}
$$

end do

\section{CMNEU AB 'RSMALL...' WEgLASSEN}

CM DY =RLARGE/FLOAT $(\mathrm{NJ}-2)$

$C M \quad Y(1)=-0.5 * D Y$

CM DO $101 \mathrm{~J}=2, \mathrm{NJ}$

CM101 $Y(J)=Y(J-1)+D Y$

CM DO $101 \mathrm{~J}=1, \mathrm{NJ}$

CM101 $Y(J)=Y(J-1) \star 0.5$

CM RSMALL $=0.5 *(Y($ JSTEP $)+Y($ JSTP 1$))$

C----DEPENDENT VARIABLE SELECTION

INCALU $=$. TRUE.

INCALV $=$. TRUE .

INCALP $=$. TRUE.

* jms

* INCALK=. TRUE.

incalk=. False.

* INCALD=.TR!'E.

incald=. false. 


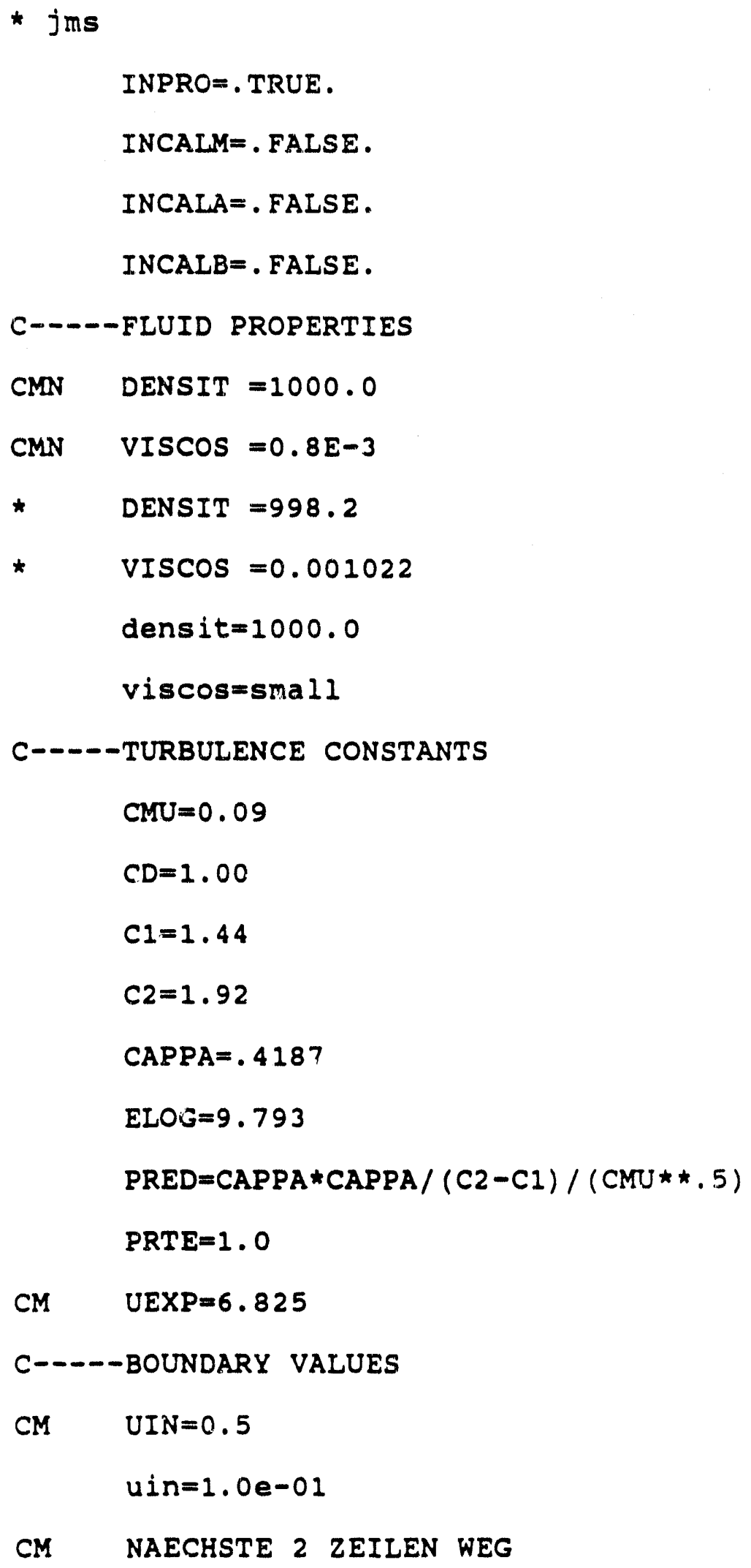




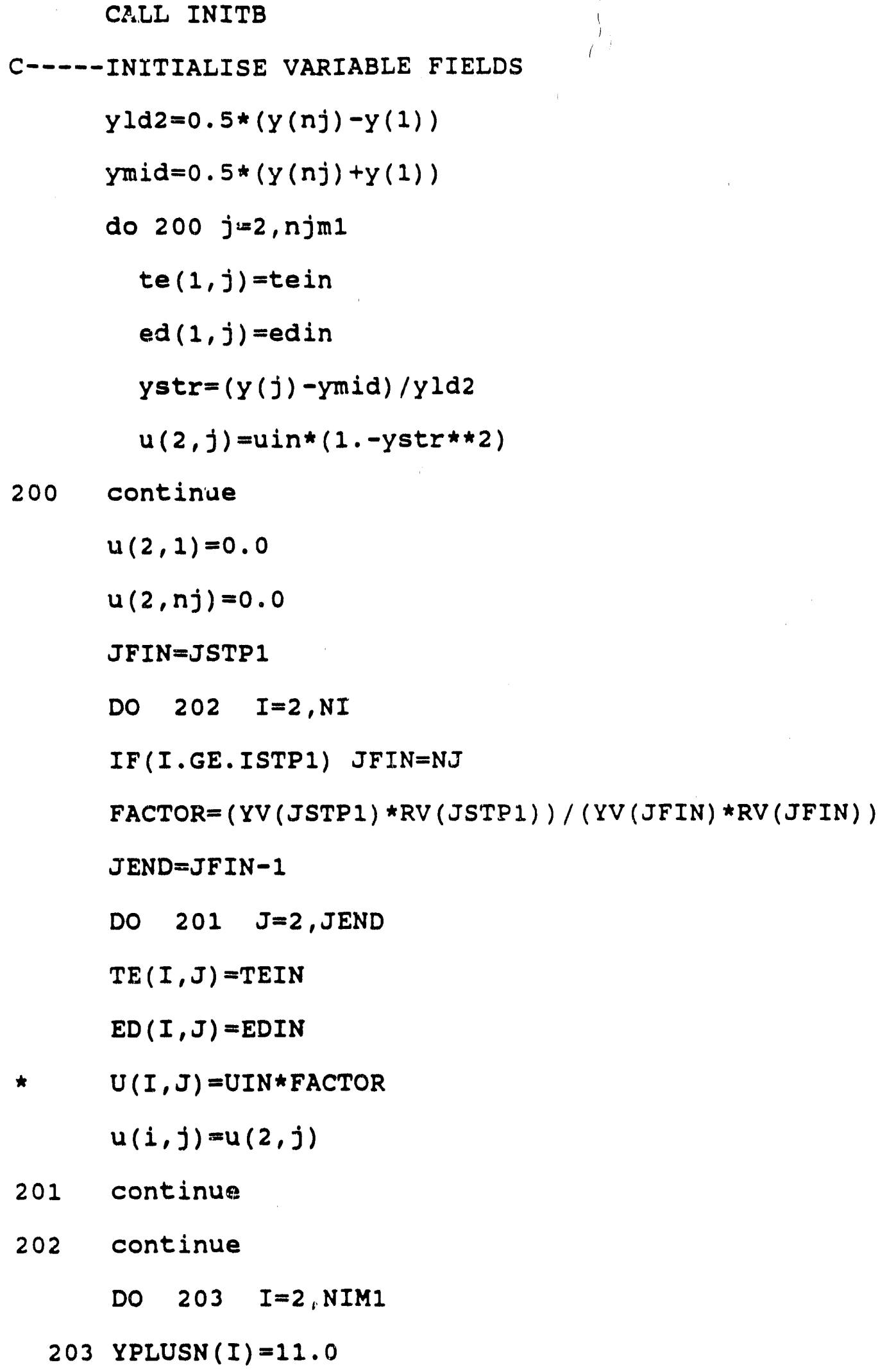




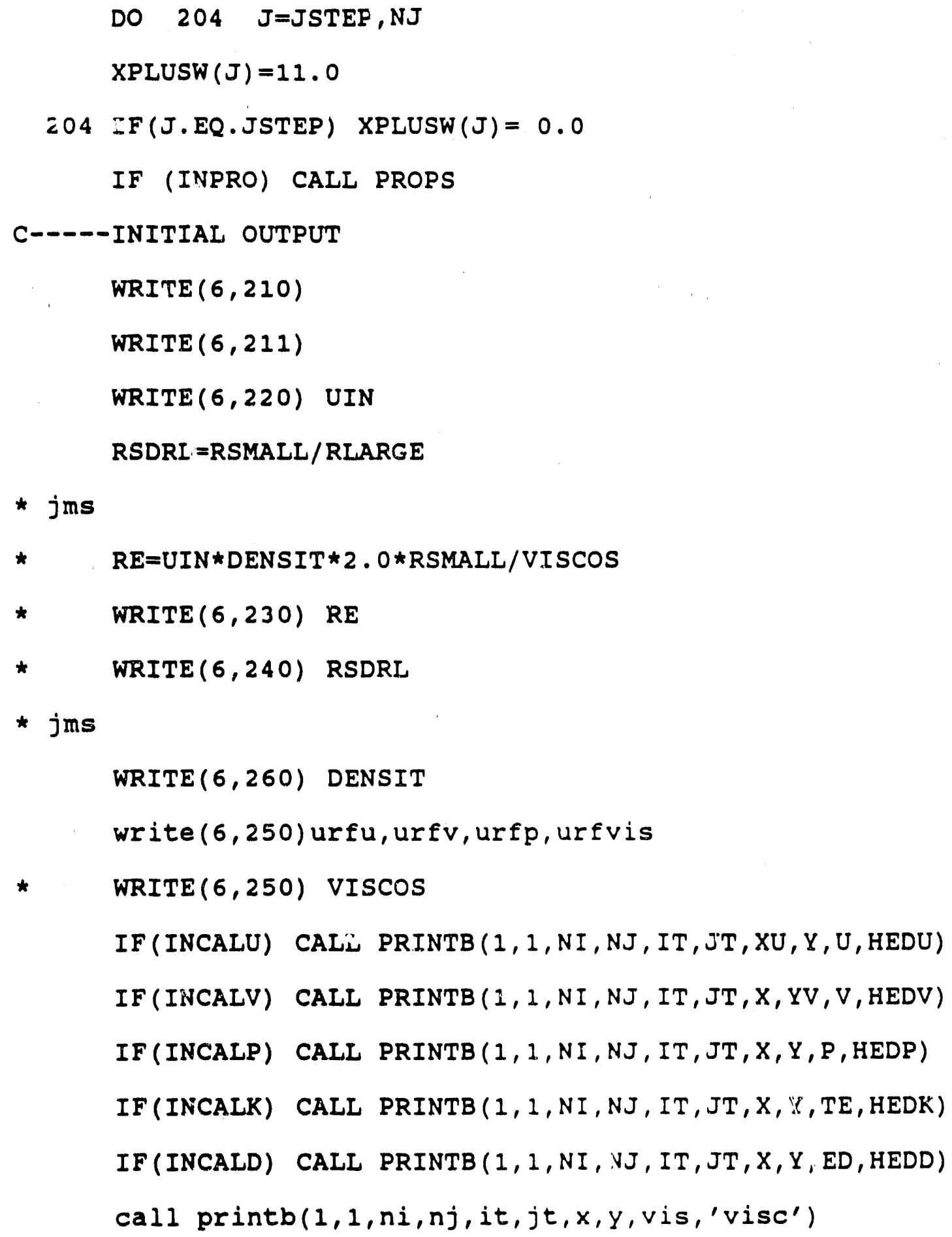

* output of grid 


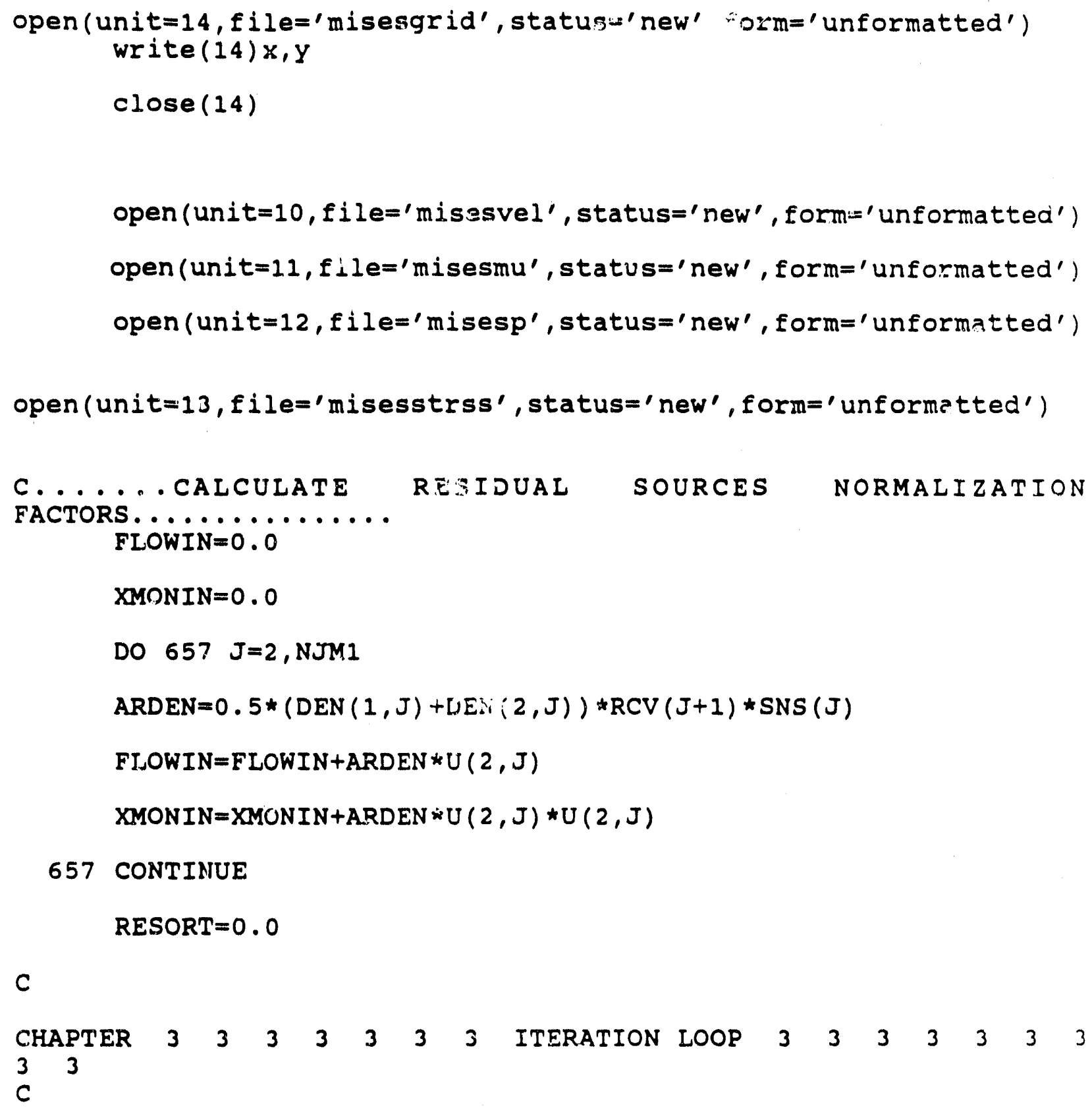




$$
\begin{aligned}
& \operatorname{vold}(i, j)=v(i, j) \\
& \operatorname{pold}(i, j)=p(i, j) \\
& \operatorname{visold}(i, j)=\operatorname{vis}(i, j)
\end{aligned}
$$

end do

end do

IF (INCALU) CALI CALCU

IF(INCALV) CALL CÁLCY

IF (INCALP) CALL CALCP

IF (INCALK) CALL CALCTE

IF (INCALD) CALL CALCED

\section{C----UPDA'TE FLUID PROPERITIES}

IF (INPRO) CALL PROPS

dum $x=0.0$

$d v m x=0.0$

dpmx $=0.0$

$d v i \operatorname{smx}=0.0$

$i c l l=0$

aveum $=0.0$

avep $=0.0$

avemu $=0.0$

do $i=1, n x$

$$
\begin{aligned}
& \text { do } j=1, \text { ny } \\
& \text { delu=abs }(u(i, j)-\operatorname{vold}(i, j)) \\
& \text { delv=abs }(v(i, j)-\operatorname{vold}(i, j)) \\
& \text { delp=abs }(p(i, j)-\operatorname{pold}(i, j)) \\
& \operatorname{delvis=abs}(\operatorname{vis}(i, j)-\operatorname{visold}(i, j))
\end{aligned}
$$


dumx=amax1 (dumx, delu)
dvmx=amax1 (dvmx, delv)
dpmx=amax1 (dpmx, delp)
dvismx=amax1 (dvismx, delvis)

if $(j \cdot g t .1$. and.j.lt.ny. and.i.gt.2. and.i.lt.nx) then

icll $=i \operatorname{cll} 1$

ave um=aveum+sqrt $(u(i, j) \star \star 2+v(i, j) \star \star 2)$

avep $=\operatorname{avep}+\operatorname{abs}(p(i, j))$

avemu=avemu+vis $(i, j)$

end if

end do

end do

aveum=aveum/float $($ icll)

avep=avep/float (icll)

avemu=avemu/float (icll)

dum $x=$ dum $x$ / aveum

$d v m x=d v m x /$ aveum

$d p m x=d p m x /$ avep

$d v i \operatorname{sm} x=d v i \operatorname{sm} x /$ avemu

C----INTERMEDIATE OUTPUT

RESORM $=$ RESORM / FLOW $\perp N$

RESORU =RESORU / XMONIN

RESORV $=$ RESORV / XMONIN

DUMMY $=0.0$

WRITE (6, 311 ) NITER, RESORU, RESORV , RESORM, RESORT, RESORK, RESORE 
write $(6,312)$ dumx, dvmx, dpmx, dvismx, a veum IF (MOD (NITER, INDPRI) . NE.0) GO TO 301

IF (INCALU) CALL PR NTB(1, 1,NI,NJ, IT, JT, XU, Y,U, HEDU) IF (INCALV) CALL PRINTB(1, 1,NI,NJ, IT, JT, X, YV,V, HEDV) IF (INCALP) CALL PRINTB (1, 1,NI,NJ,IT,JT, X, Y, P, HEDP) IF (INCALK) CALL PRINTB (1, 1,NI,NJ, IT, JT, X, Y, TE, HEDK) IF (INCALD) CALL PRINTB(1,1,NI, NJ, IT, JT, X, Y, ED, HEDD) call printb $\left(1,1, n i, n j, i t, j t, x, y, v i s,{ }^{\prime} v i s c^{\prime}\right)$ WRITE $(6,310)$ IMON, JMON

* plotting of velocity write (10) niter, $u, v$

* plotting of viscosity write(11) niter, vis

* plotting of pressure write (12) niter, p

* plotting of shear stress do $i=2, \operatorname{nim} 1$ do $j=2, n j m 1$

* velocity gradients

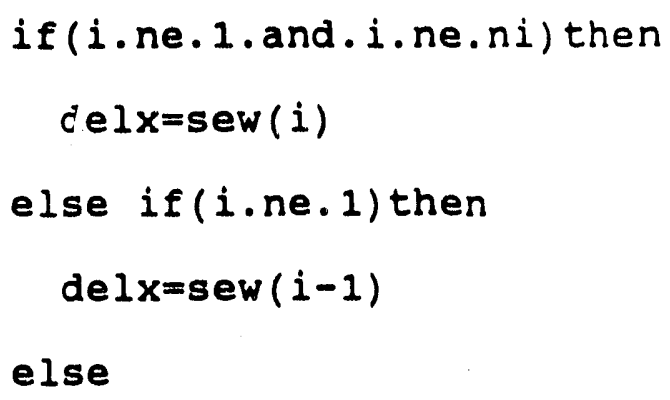




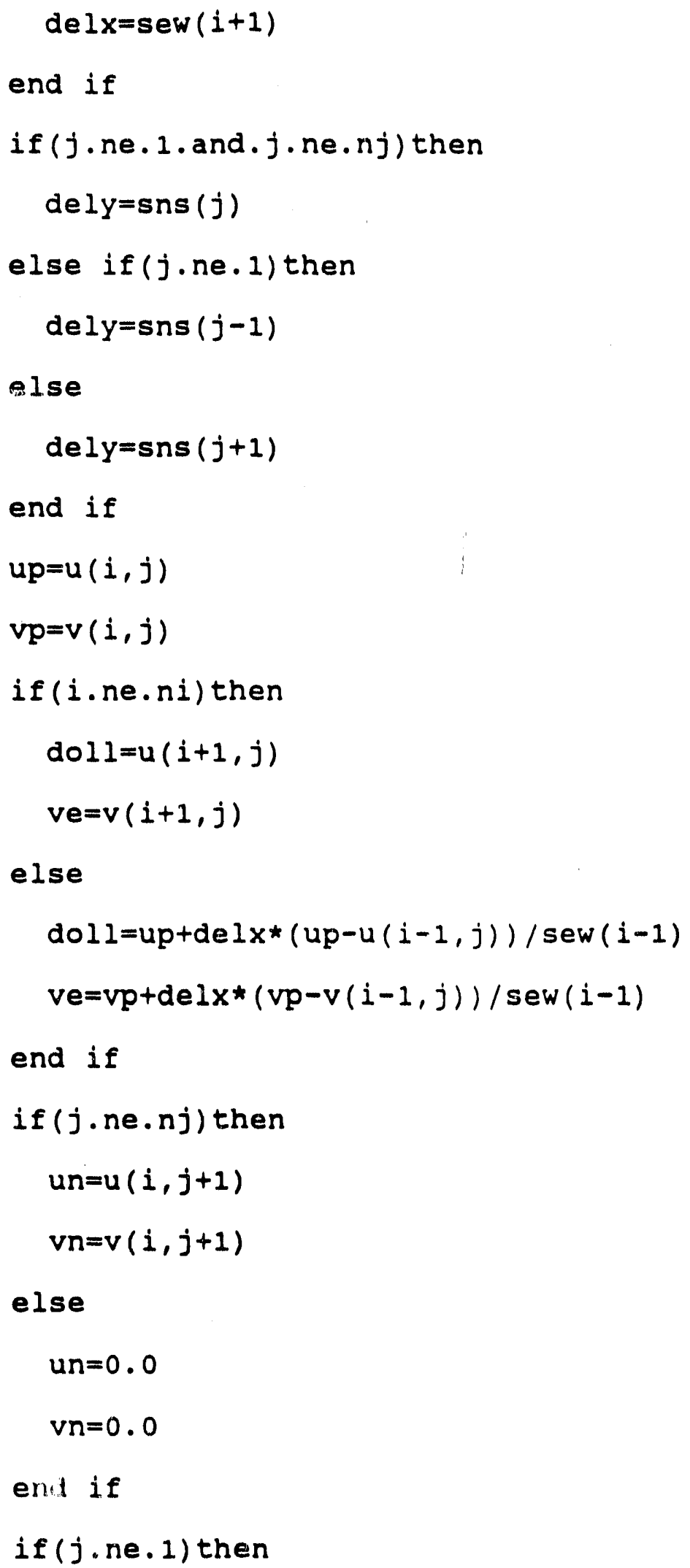




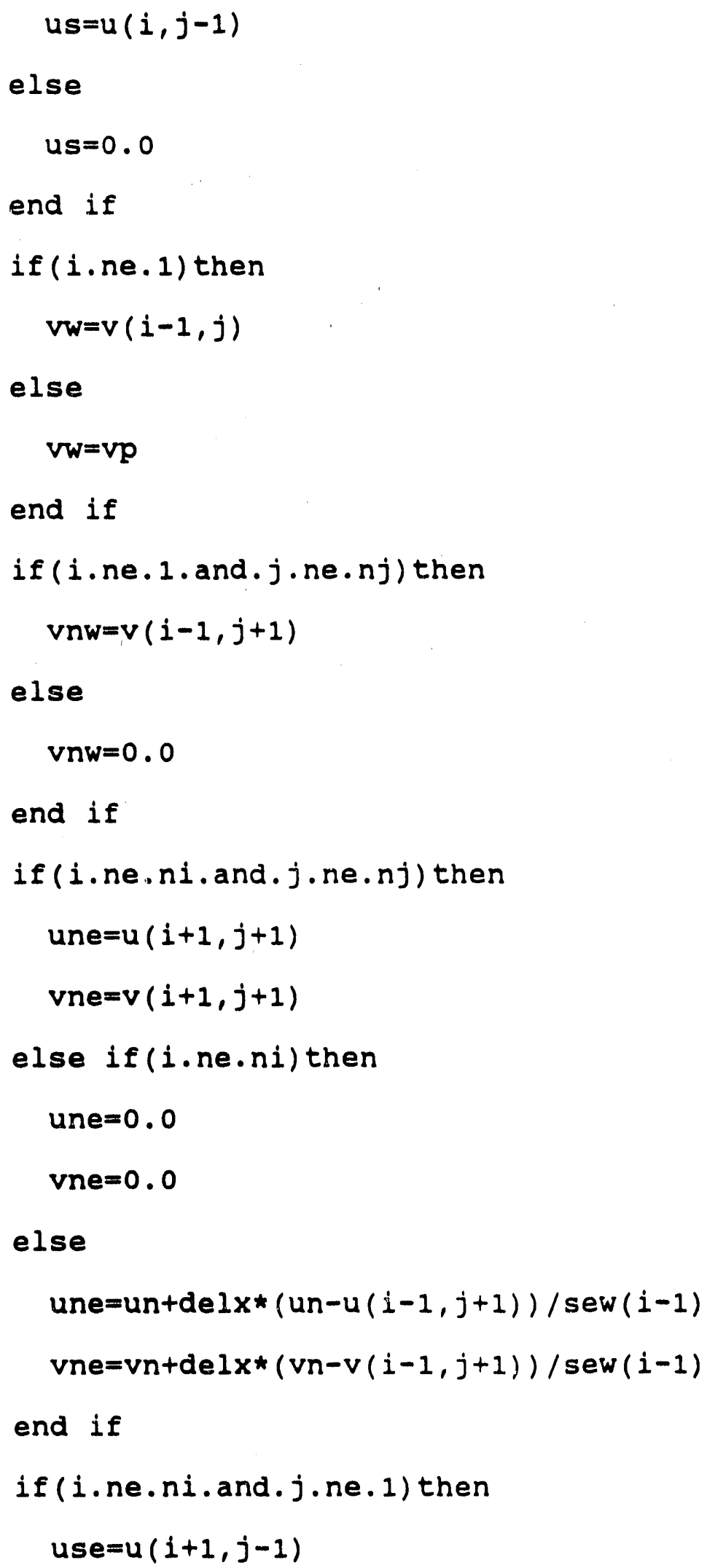




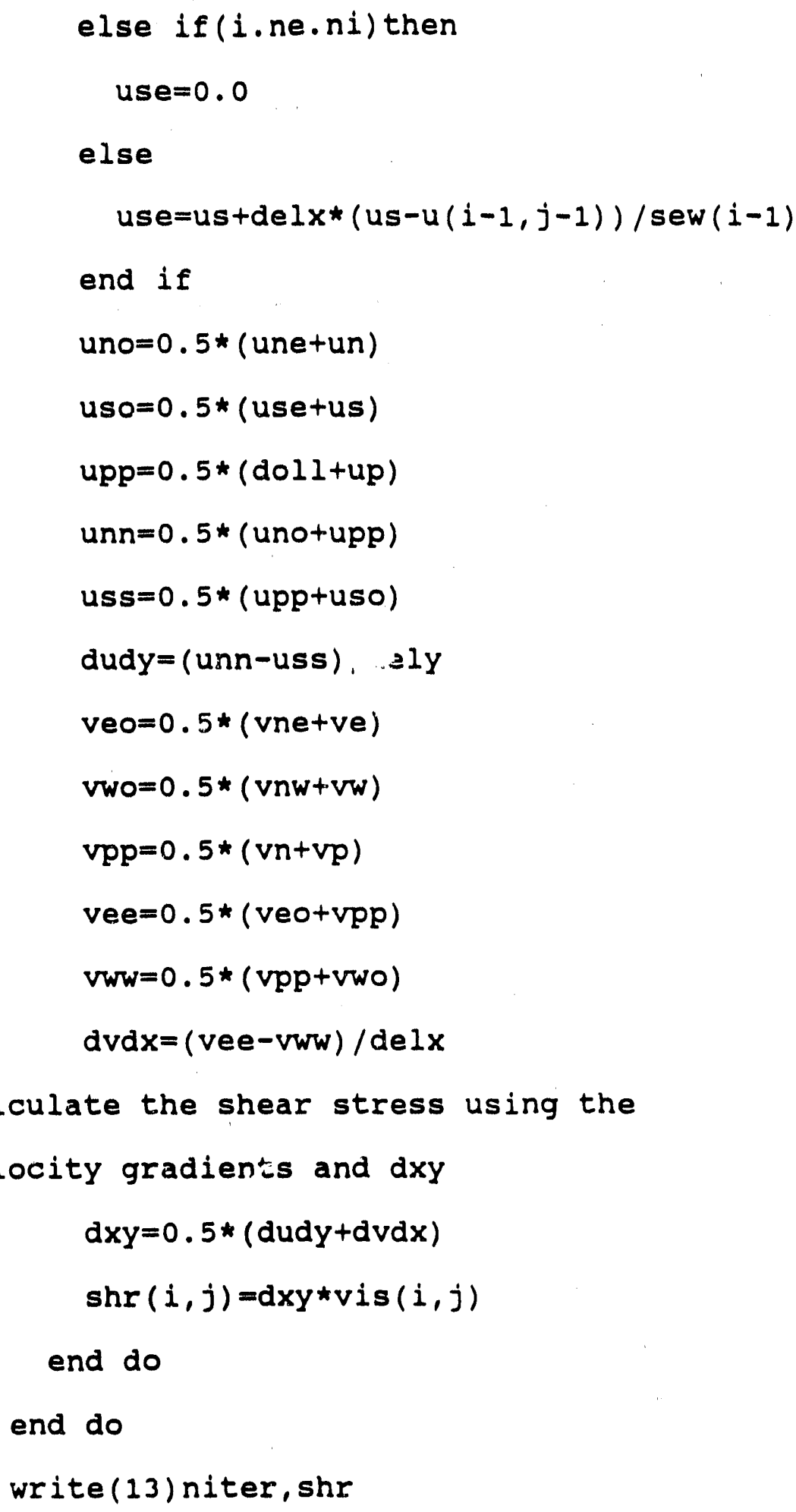

301 CONTINUE 
C-----TERMINATION TESTS

SORCE =AMAXI (RESORM, RESORU, RESORV)

* IF (NITER.EQ.20.AND.SORCE.GT.1.0E4*SORMAX) GO TO 302

IF (NITER.EQ.MAXIT) GO TO 30 ?

IF (SORCE.GT.SORMAX) GO TO 300

302 CONTINUE

C

$\begin{array}{lllllllllllll}\text { CHAPTER } & 4 & 4 & 4 & 4 & 4 & 4 & \text { FINAL OPERATIONS AND OUTPUT } & 4 & 4 & 4 & 4\end{array}$ 44

if $(\bmod (n i t e r$, indpri) ne.0) then

* final printouts

if(incalu) call printb(1, 1, ni, nj, it, jt, $x u, y, u$, hedu)

if(incalv) call printb(1,1,ni,nj, it,jt, $x, y v, v$, hedv)

if (incalp) call printb(1,1,ni,nj,it,jt, $x, y, p$, hedp)

if(incalk) call printb(1,1,ni,nj,it,jt, $x, y, t e, h e d k$ )

if(incald) call printb(1, $1, n i, n j, i t, j t, x, y$, ed, hedd)

call printb(1,1,ni,nj,it,jt, $\left.x, y, v i s, v^{\prime} s^{\prime}\right)$

* plotting of velocity

write(10) niter, u,v

* plotting of viscosity

write(11)niter, vis

* plotting of pressure

write(12) niter, p

* plotting of shear stress

do $i=2$, niml 
do $j=2, n j m 1$

* velocity gradients

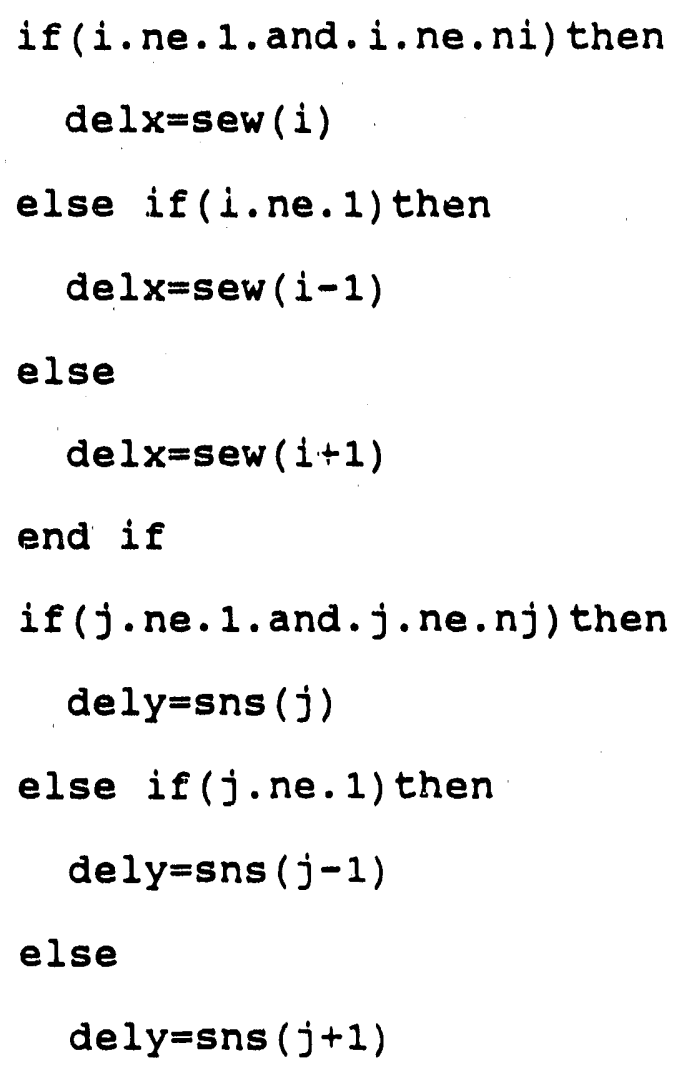




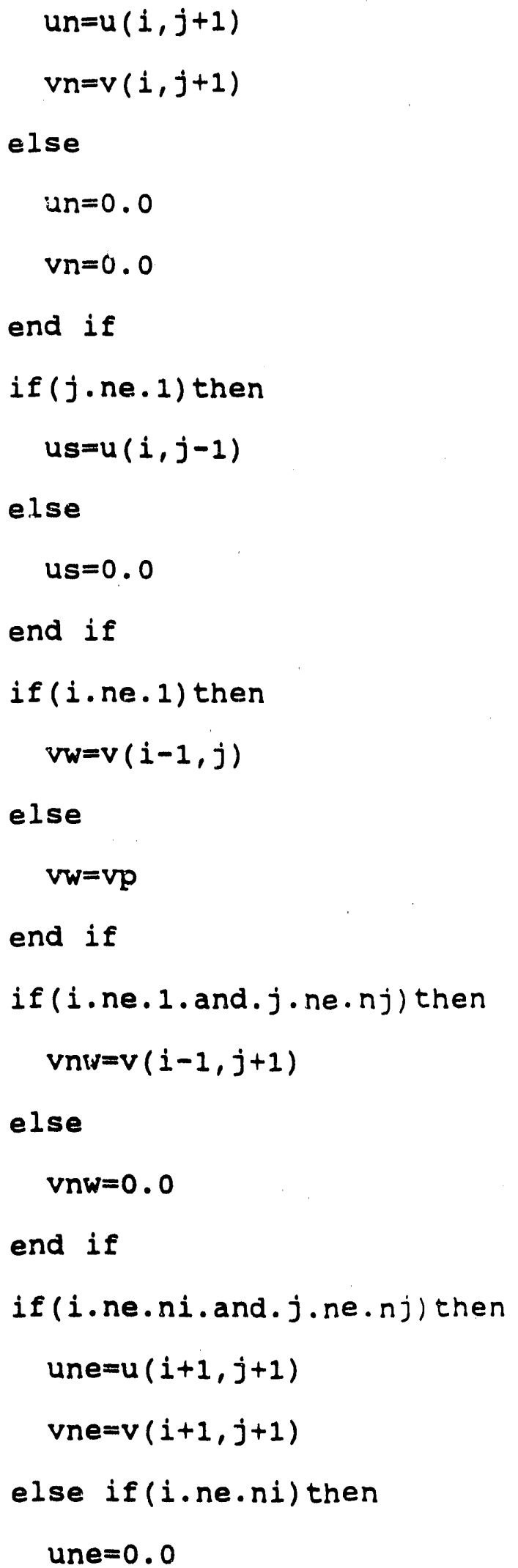




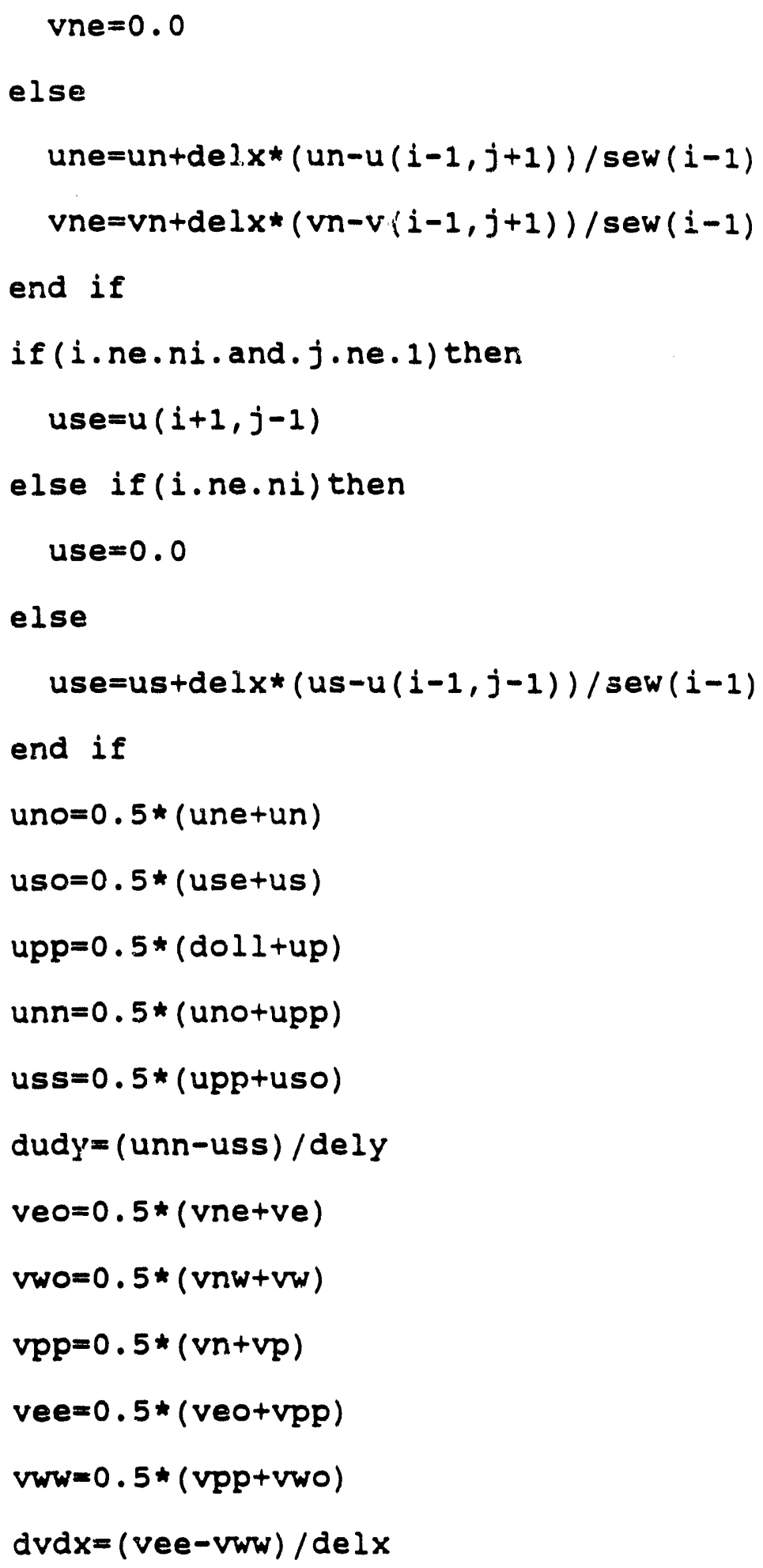




$$
\begin{aligned}
& d x y=0.5 *(d u d y+d v d x) \\
& \operatorname{shr}(i, j)=d x y * v i s(i, j)
\end{aligned}
$$

end do

end do

write (13) niter, shr

end if

close (10)

close (11)

close (12)

close (13)

STOP

C----FORMAT STATEMENTS

210 FORMAT()

211 FORMAT()

220 FORMAT(1HO, 15X,'INLET FLUID VELOCITY', T60,1H=,3X, 1PE11.3)

230 FORMAT (1H0, 15X,'REYNOLDS NUMBER ', T60, 1H=,3X, 1PE11.3)

240 FORMAT (1HO, 15X, 'DIAMETER RATIO ',T60,1H=,3X,1PE11.3)

* 250 Format (1H0, 15X,'LAMINAR VISCOSITY', T60,1H=,3X, 1PE11.3)

250 format $\left({ }^{\prime} 0^{\prime}, 15 x,{ }^{\prime}\right.$ URFU ', t60,' $=$ ', 3x, 1pg11.3, /,

$1 \quad 0^{\prime}, 15 x, '$ URFV ',t60,' =',3x, 1pg11.3,1,

$2 \quad 0^{\prime}, 15 x$, URFP $^{\prime}, \mathrm{t} 60,{ }^{\prime}=\prime, 3 x, 1 \mathrm{pg} 11.3,1$,

3

$' 0^{\prime}, 15 x, '$ URFVIS ',t60,'=',3x,1pg11.3)

260 FORMAT (1H0, 15X,'FLUID DENSITY ', T60, 1H=,3X,1PE11.3)

310 FORMAT (1HO,'ITER SOURCE SUM

$15-0-0-0-0-I$ LOCATION', '

', I-I-----FIELD VALUES AT MONITORING 


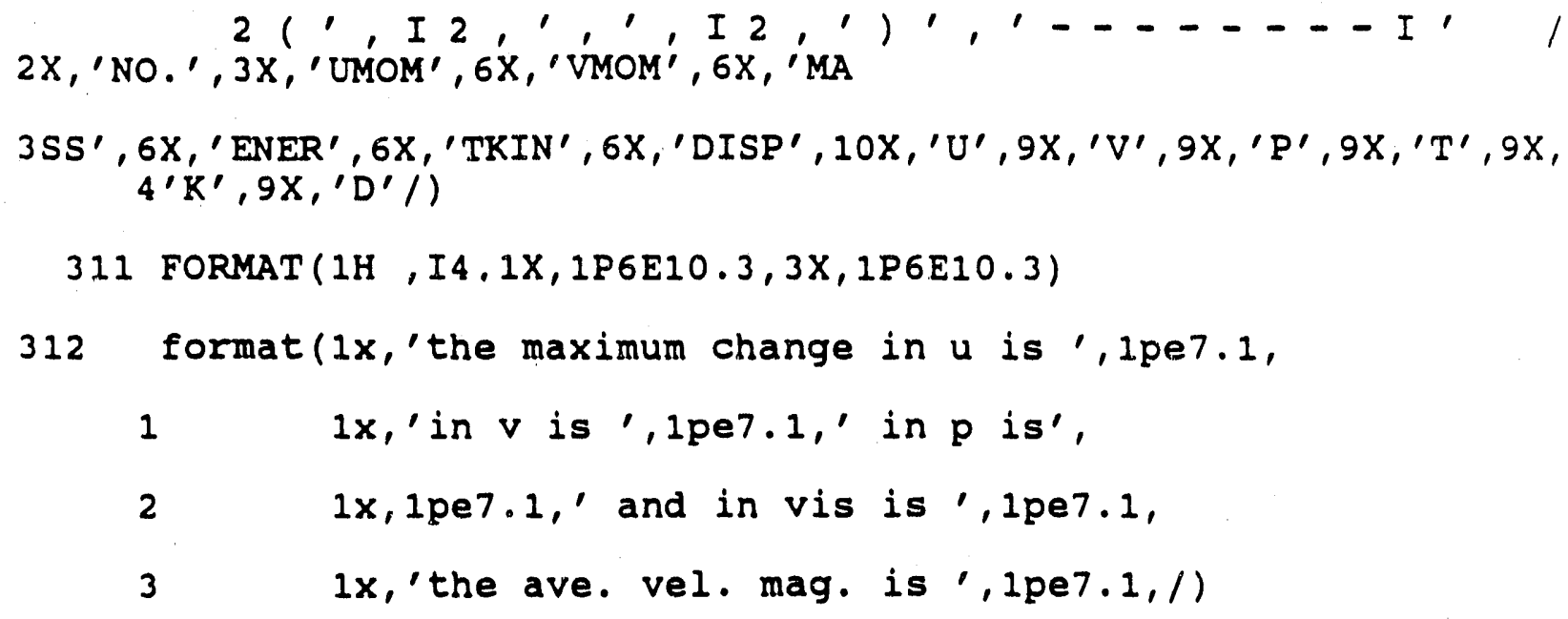

311 FORMAT (1H, I4, 1X, 1P6E10.3, 3X, 1P6E10.3)

312 format (1x,'the maximum change in $u$ is ', 1pe7.1,

$1 \quad 1 x$, 'in $v$ is ', 1pe7.1,' in $p$ is',

$21 \times, 1$ pe $7.1, '$ and in vis is ', 1pe7.1,

$31 x$, 'the ave. vel. mag. is ', 1pe7.1,/)

$402 \operatorname{FORMAT}(/ / / 5 \mathrm{X}, 1 \mathrm{HI}, 7 \mathrm{X}, 5 \mathrm{HXU}(\mathrm{I}), 6 \mathrm{X}, 10 \mathrm{HS} . \mathrm{S}$. COEFF. $)$

403 FORMAT (/5X, I5, 2(1PE11.3))

404 FORMAT $(/, " \star \star$ SOLUTION DIVERGES OR NEEDS MORE ITERATIONS

$1, / 1$

END

SUBROUTINE PROPS

C

PARAMETER $(\mathrm{NX}=50)$

PARAMETER (NY=26)

$\begin{array}{llllllllllllllll}\text { CHAPTER } & 0 & 0 & 0 & 0 & 0 & 0 & 0 & 0 & \text { PRELIMINARIES } & 0 & 0 & 0 & 0 & 0 & 0\end{array}$ 00

c

\section{COMMON}

1/FLUPR/URFVIS, VISCOS, DENSIT , PRANDT, DEN (NX, NY), VIS (NX, NY)

$1 / V A R / U(N X, N Y), V(N X, N Y), P(N X, N Y), P P(N X, N Y), T E(N X, N Y), E D(N X, N Y)$

* $\quad 1 / A L L / I T, J T, N I, N J, N I M 1, N J M 1$, GREAT

1/all/it,jt, ni, nj, niml, njm1, great, small

$1 /$ TURB/GEN (NX, NY) , CD , CMU, C1 , C2, CAPPA, ELOG, PRED , PRTE , PRM

* need geometry to calculate deformation rate tensor 
$1 / \operatorname{geom} / x(n x), y(n y), \operatorname{dxep}(n x)$, dxpw $(n x)$, dynp (ny), dyps (ny),

$\operatorname{sns}(n y), \operatorname{sew}(n x), x u(n x), y v(n y), r(n y), r v(n y), r c v(n y)$, indocos

C

$\begin{array}{llllllll}\text { CHAPTER } & 1 & 1 & 1 & \text { VISCOSITY } & 1 & 1 & 1\end{array}$

c

* material constant $k$

data rkysf/1.0/

* calculate granular dynamic viscosity

do $101 \mathrm{i}=1, \mathrm{ni}$

do $100 j=1, n j$

* velocity gradients

if ( $i$.ne.1.and.i.ne.ni) then

del $x=\operatorname{sew}(i)$

else if(i.ne.1) then

$\operatorname{del} x=\operatorname{sew}(i-1)$

else

del $x=\operatorname{sew}(i+1)$

end if

if (j.ne.1.and.j.ne.nj) then

dely=sns $(j)$

else if (j.ne.1) then

$\operatorname{del} y=\operatorname{sns}(j-1)$

else

$\operatorname{del} y=\operatorname{sns}(j+1)$

end if

$u p=u(i, j)$ 


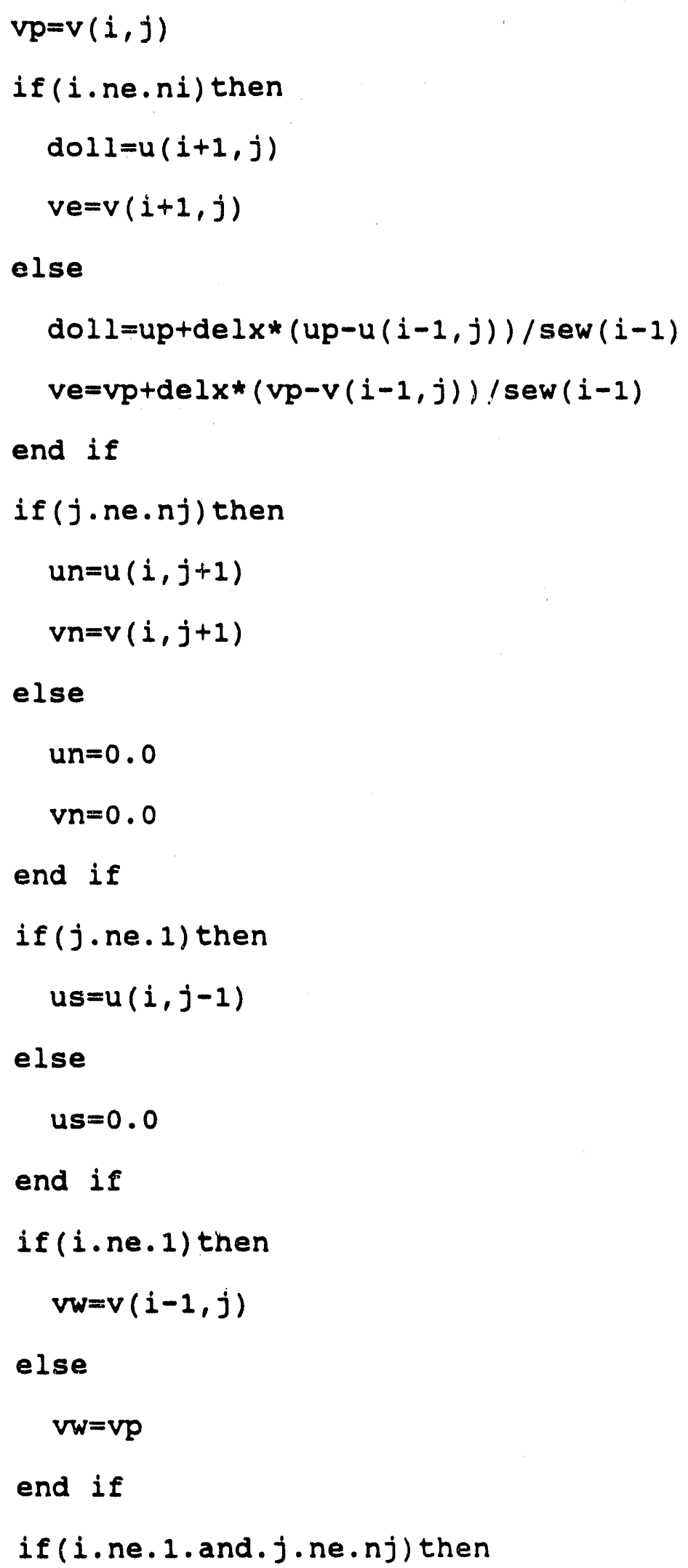




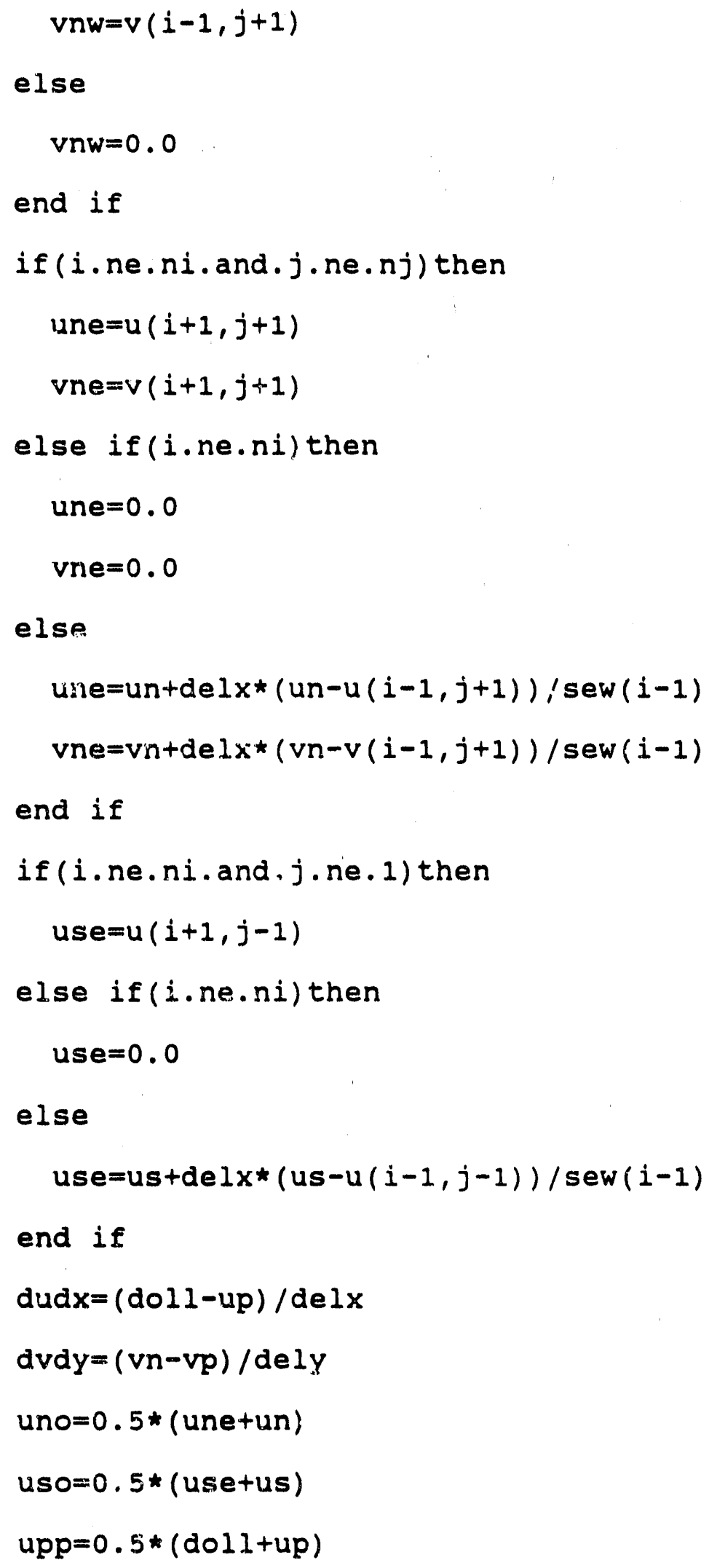




$$
\begin{aligned}
& \text { unn }=0.5 *(\text { unotupp }) \\
& \text { uss }=0.5 *(\text { upp }+u s o) \\
& \text { dudy }=(\text { unn-uss }) / d e l y \\
& \text { veo }=0.5 *(\text { vne }+v e) \\
& \text { vwo }=0.5 *(v n w+v w) \\
& \text { vpp }=0.5 *(v n+v p) \\
& \text { vee }=0.5 *(v e o+v p p) \\
& \text { vww }=0.5 *(v p p+v w o) \\
& d v d x=(v e e-v w w) / d e l x
\end{aligned}
$$

* second invariant of the deformation rate tensor

$d 2 d=-((d u d x-d v d y) * \star 2+d v d y * \star 2+d u d x * \star 2) / 6 .-(0.5 *(d u d y+d v d x)) * \star 2$

* plastic scalar at $i, j$

$$
q=\operatorname{sqr} t(-d 2 d)
$$

* effective granular dynamic viscosity at $i, j$

$$
\begin{aligned}
& \text { if }(q \cdot g t . \text { small }) \text { then } \\
& \quad \text { visnew }=0.5 * \text { rkysf } / q \\
& \text { else }
\end{aligned}
$$

* static case

$$
\text { visnew }=0.5 * \text { rkysf } / \text { small }
$$

end if

$$
\operatorname{vis}(i, j)=\operatorname{urfvis} * v i s n e w+(1 .-\operatorname{urfvis}) \star v i s(i, j)
$$

100 continue

101 continue

RETURN

END

SUBROUTINE PROMOD 
C

$\begin{array}{llllllllllllllll}\text { CHAPTER } & 0 & 0 & 0 & 0 & 0 & 0 & 0 & \text { PRELIMINARIES } & 0 & 0 & 0 & 0 & 0 & 0 & 0\end{array}$ 00

C

PARAMETER $(\mathrm{NX}=50)$

PARAMETER $(\mathrm{NY}=26)$

COMMON

1/UVEL/RESORU, NSWPU, URFU, DXEPU (NX) , DXPWU (NX) , SEWU (NX)

1/VVEL/RESORV, NSWPV, URFV, DYNPV (NY) , DYPSV (NY) , SNSV (NY)

1/PCOR/RESORM, NSWPP, URFP, DU (NX, NY) , DV (NX, NY) , IPREF, JPREF

$1 / V A R / U(N X, N Y), V(N X, N Y), P(N X, N Y), P P(N X, N Y), T E(N X, N Y), E D(N X, N Y)$

* $\quad 1 / A L L / I T, J T, N I, N J, N I M 1, N J M 1$, GREAT

1/all/it,jt,ni,nj, nim1,njm1, great, small

$1 / G E O M / X(N X), Y(N Y), D X E P(N X), D X P W(N X), D Y N P(N Y), D Y P S(N Y)$ ，

$S N S(N Y), S E W(N X), X U(N X), Y V(N Y), R(N Y), R V(N Y), R C V(N Y), \stackrel{1}{1}$ NDCOS

$1 /$ IPOL/FXP (NX), FXM(NX), FYP(NY) , FYM(NY)

1/FLUPR/URFVIS, VISCOS, DENSIT, PRANDT, DEN (NX, NY) ,VIS (NX, NY)

1/KASE T1/UIN, TEIN, EDIN, FLOWIN, ALAMDA,

RSMALL, RLARGE , AL1, AL2, JSTEP, ISTEP, JSTP1, JSTM1, ISTP1, ISTM1

$1 / \operatorname{SUSP} / \operatorname{SUKD}(N X, N Y), \operatorname{SPKD}(N X, N Y)$

$1 / C O E F / A P(N X, N Y), A N(N X, N Y), A S(N X, N Y), A E(N X, N Y), A W(N X, N Y), S U(N X, N Y)$, $1 \quad S P(N X, N Y)$

$1 /$ TURB/GEN (NX, NY) , CD , CMU, C1, C2 , CAPPA, ELOG , PRED , PRTE , PRM

$1 /$ WALLF/YPLUSN (NX) , XPLUSW (NY) , TAUN (NX) , TAUW (NY)

C

$\begin{array}{llllllllllllllllll}\text { CHAPTER } & 1 & 1 & 1 & 1 & 1 & 1 & 1 & 1 & \text { PROPERTIES } & 1 & 1 & 1 & 1 & 1 & 1 & 1\end{array}$ 11

C 


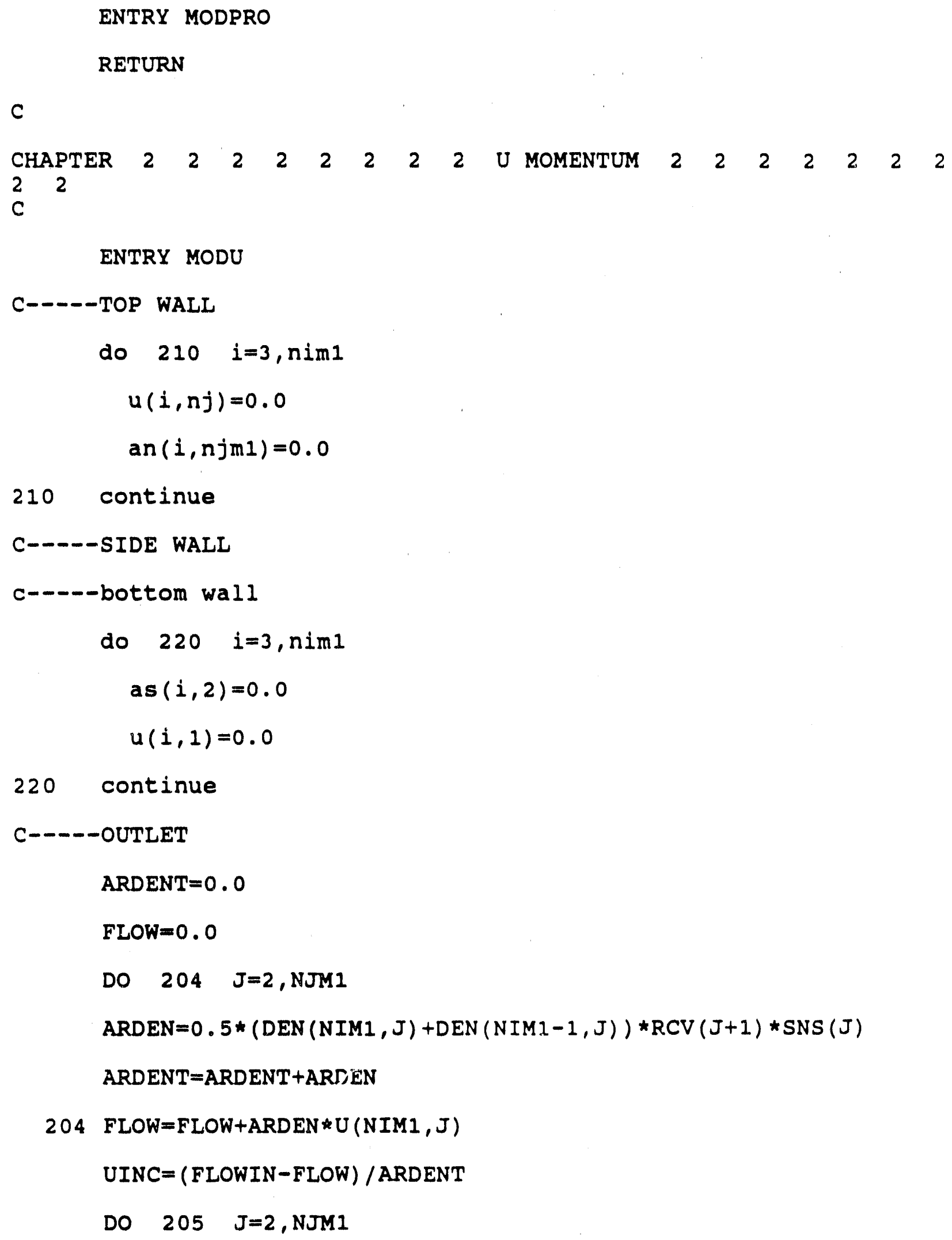




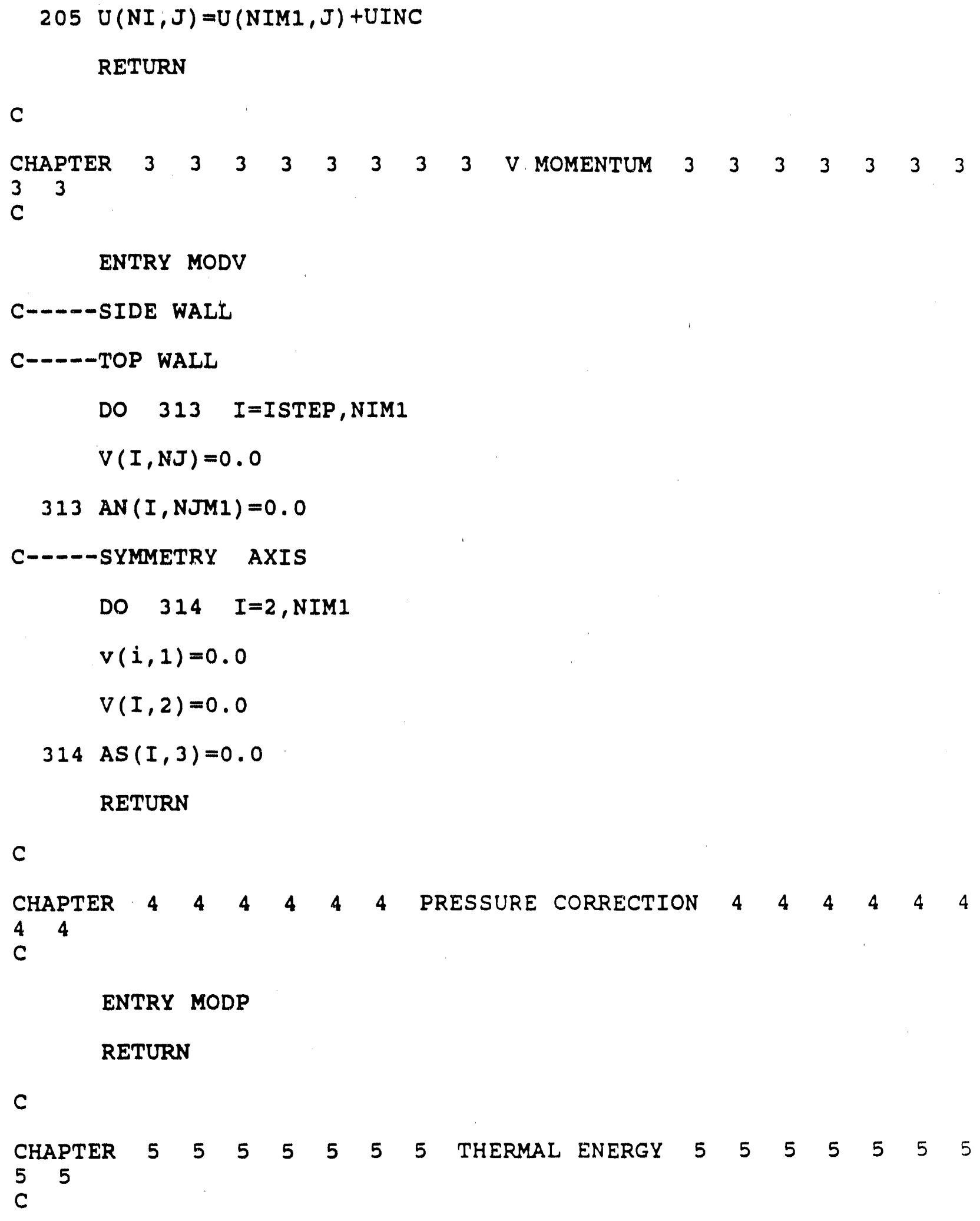

ENTRY MODT 
C----NO MODIFICATIONS FOR THIS PROBLEM

RETURN

C

CHAPTER $6 \begin{array}{lllllllll}6 & 6 & 6 & 6 & 6 & 6 & 6 & \text { TURBULENT KINETIC ENERGY }\end{array}$

C

ENTRY MODTE

C----TOP WALL

CDTERM $=C M U * \star 0.25$

$Y P=Y V(N J)-Y(N J M 1)$

$\mathrm{J}=\mathrm{N} J \mathrm{M} 1$

DO $610 \quad I=2, N I M 1$

$D E N U=D E N(I, J)$

$\operatorname{SQRTK}=\operatorname{SQRT}(\mathcal{T}(I, J))$

$\operatorname{VOL}=\operatorname{RCV}(J+1) * \operatorname{SNS}(J) * \operatorname{SEW}(I)$

$\operatorname{GENCOU}=(\operatorname{ABS}(\operatorname{TAUN}(I+1) * U(I+1, J) * F X M(I))+\operatorname{ABS}(T A U N(I) * U(I, J)$ * $\operatorname{IFXP}(I))$ ) / YP

YPLUSN,$I)=D E N U \star S Q R T K * C D T E R M * Y P / V I S C O S$

$\operatorname{DUDY}=((U(I, J)+U(I+1, J)+U(I, J+1)+U(I+1, J+1)) / 4 .-(U(I, J)+U(I+1, J)+$ $1 U(I, J-1)+U(I+1, J-1)) / 4$. $) / \operatorname{SNS}(J)$

$\operatorname{GENRES}=\operatorname{GEN}(I, J)-\operatorname{VIS}(I, J) \star D U D Y \star \star 2$

$\operatorname{GEN}(I, J)=$ GENRES + GENCOU

IF(YPLUSN(I).LE.11.63) GO TO 611

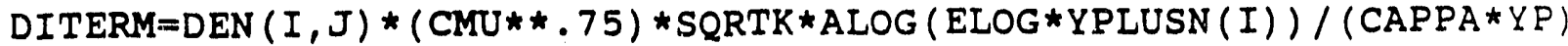
GO TO 612

611 CONTINUE

$\operatorname{DITERM}=\operatorname{DEN}(I, J) \star(C M U \star \star .75) \star S Q R T K \star Y P L U S N(I) / Y P$ 
612 CONTINUE

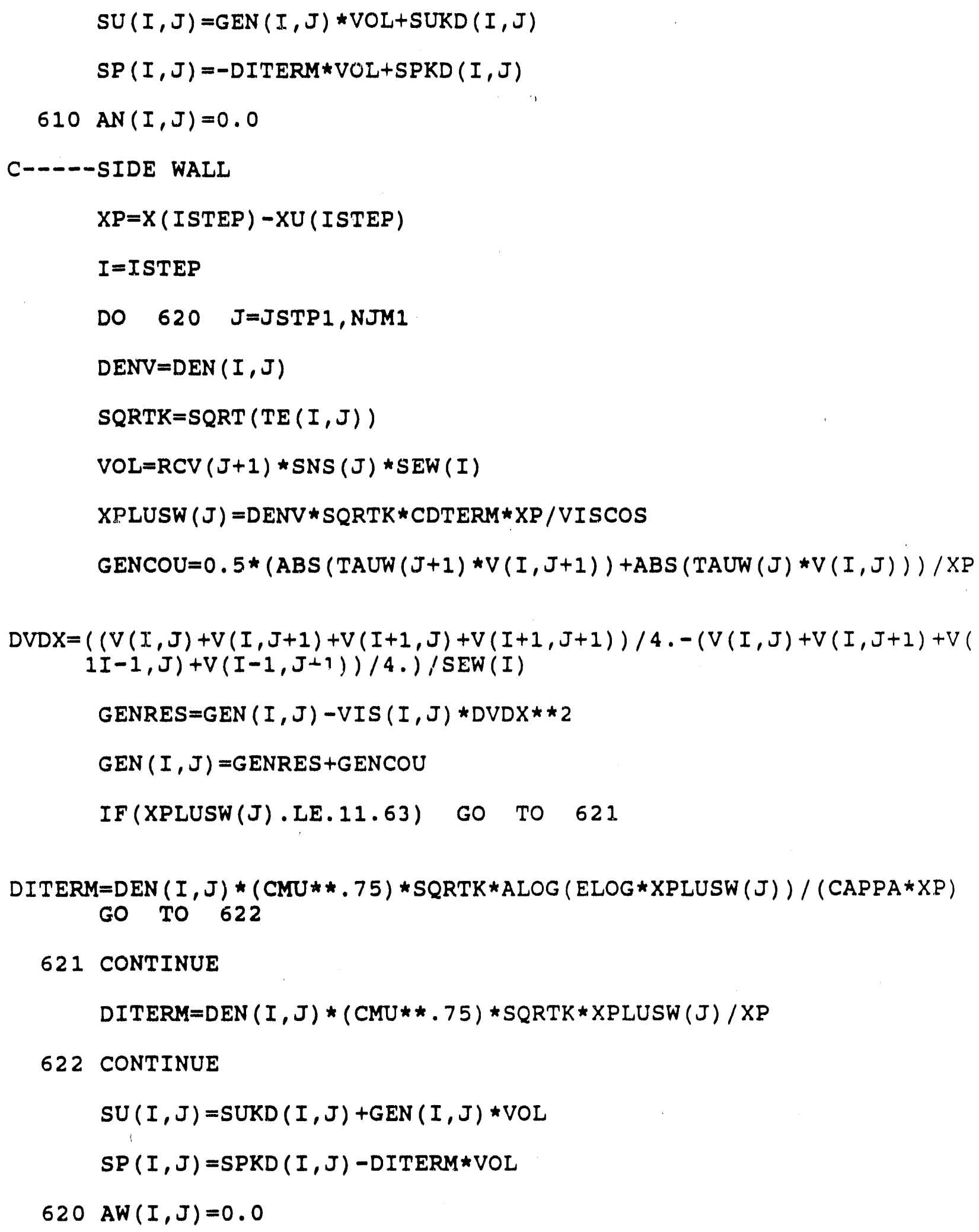




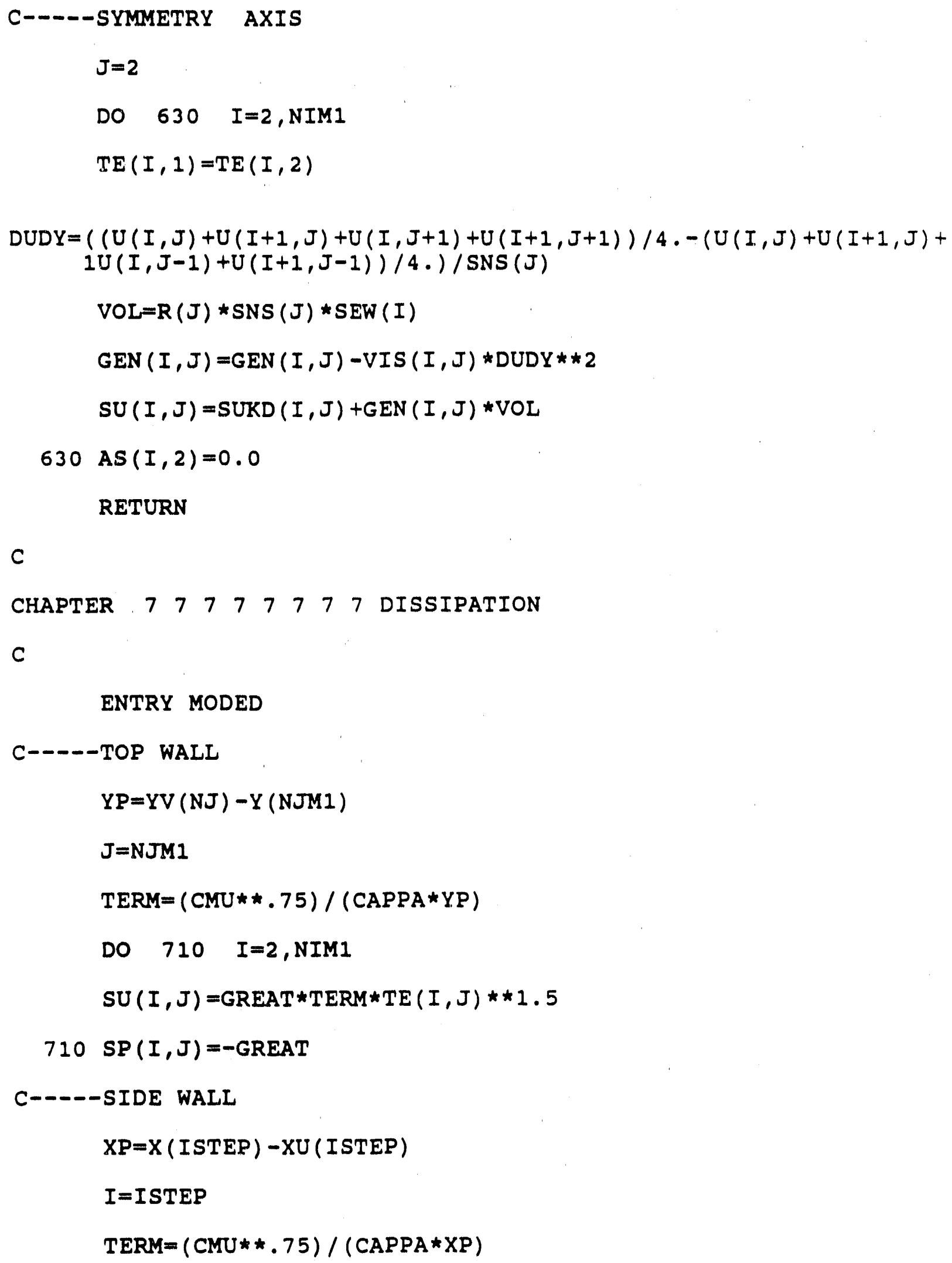




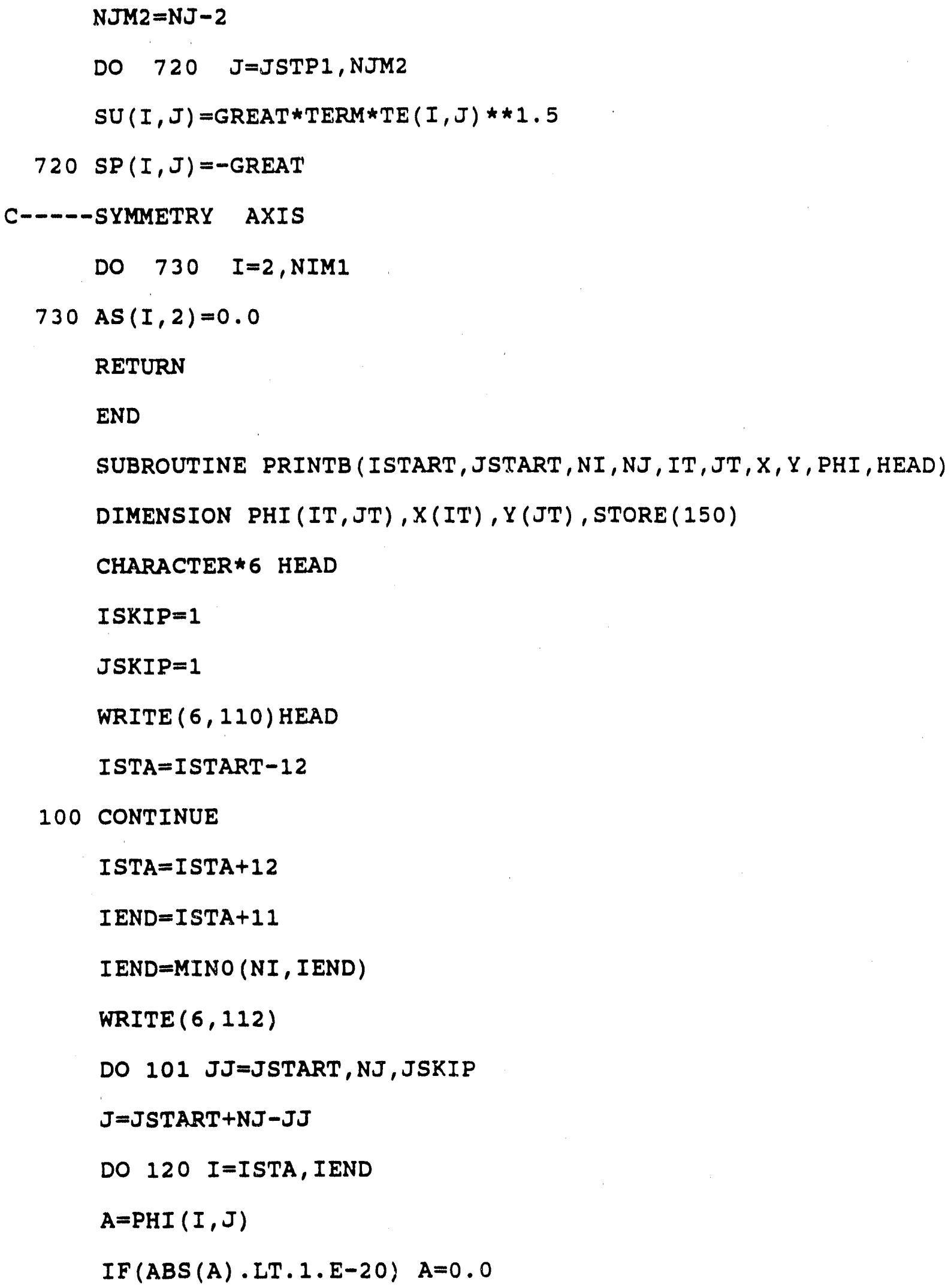




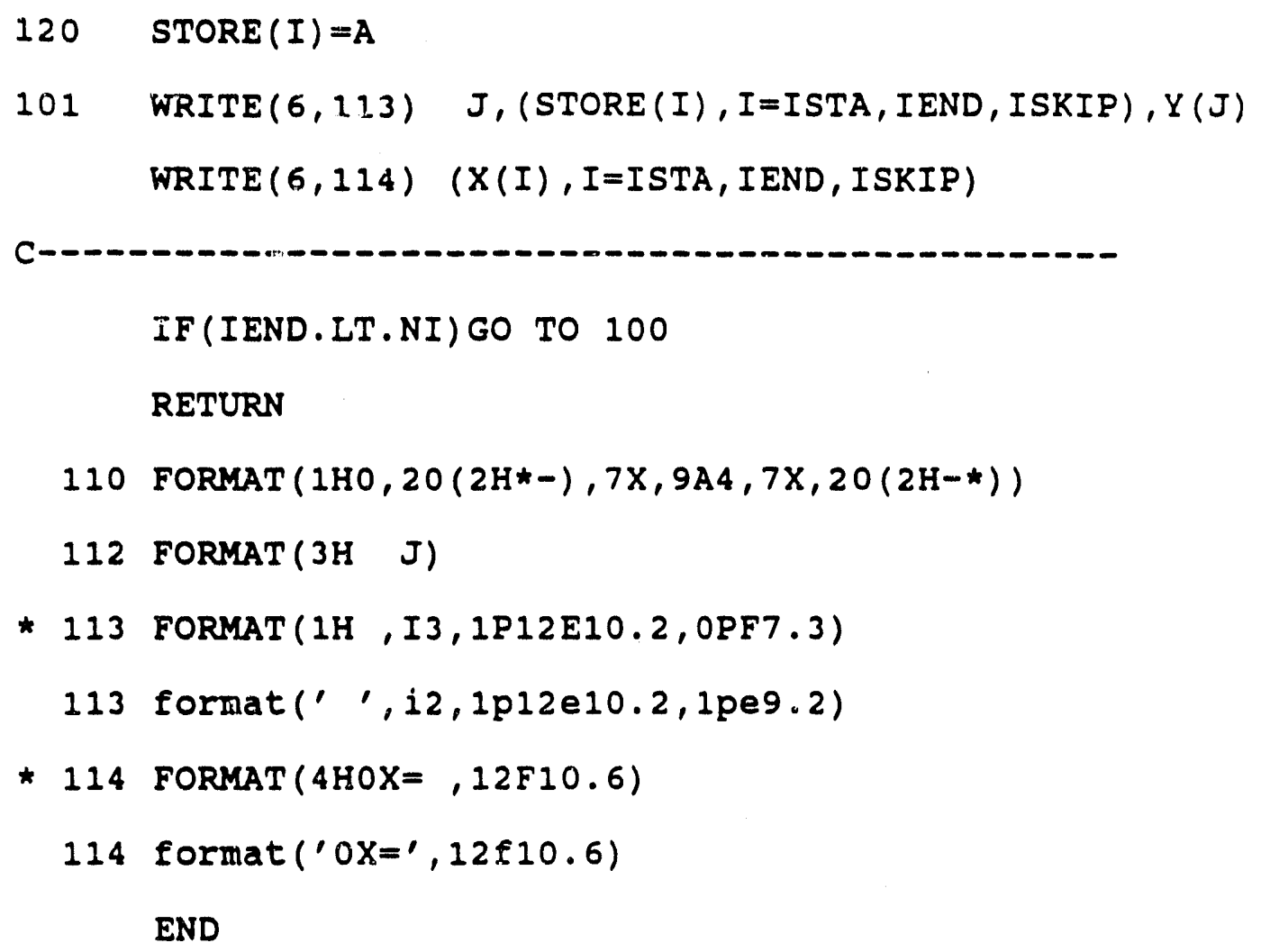


$A(J \operatorname{STM} 1)=0.0$

C-----COMMENCE W-E SWEEP

DO 100 I=ISTART, NIMI

$C(J S T M 1)=P H I(I, J S T M 1)$

C-----COMMENCE S-N TRAVERSE

DO $101 \mathrm{~J}=$ JSTART, NJM1

C-----ASSEMBLE TDMA COEFFICIENTS

$A(J)=A N(I, J)$

$B(J)=A S(I, J)$

$C(J)=A W(I, J) * \operatorname{PHI}(I-I, J)+A E(I, J) * \operatorname{PHI}(I+I, J)+S U(I, J)$

$D(J)=A P(I, J)$

C---CALCULATE COEFFICIENTS OF RECURRENCE FORMULA

$\operatorname{TERM}=1 \cdot /(D(J)-B(J) \star A(J-1))$

$A(J)=A(J) \star T E R M$

$101 C(J)=(C(J)+B(J) * C(J-1)) \star T E R M$

C-----OBTAIN NEW PHIES

DO 102 JJ=JSTART, NJM1

$\mathrm{J}=\mathrm{N} \mathrm{J}+\mathrm{JSTM} 1-\mathrm{J} \mathrm{J}$

$102 \operatorname{PHI}(I, J)=A(J) * \operatorname{PHI}(I, J+1)+C(J)$

100 CONTINUE

RETURN

END

SUBROUTINE INITB

C

$\begin{array}{llllllllllllllll}\text { CHAPTER } & 0 & 0 & 0 & 0 & 0 & 0 & 0 & 0 & \text { PRELIMINARIES } & 0 & 0 & 0 & 0 & 0 & 0\end{array}$ 00 C 
PARAMETER $(\mathrm{NX}=50)$

PARAMETER $(N Y=26)$

COMMON

1/UVEL/RESORU, NSWPU, URFU, DXEPU (NX), DXPWU (NX) , SEWU (NX)

1/VVEL/RESORV, NSWPV, URFV, DYNPV (NY) , DYPSV (NY) , SNSV (NY)

1/PCOR/RESORM, NSWPP, URFP, DU (NX, NY) , DV (NX, NY) , IPREF, JPREF

$1 / V A R / U(N X, N Y), V(N X, N Y), P(N X, N Y), P P(N X, N Y), T E(N X, N Y), E D(N X, N Y)$

* 1/ALL/IT, JT, NI, NJ , NIMI, NJMI , GREAT

1/all/it,jt,ni,nj,nim1,njm1, great, small

$1 / G E O M / X(N X), Y(N Y), D X E P(N X), D X P W(N X), D Y N P(N Y), D Y P S(N Y)$,

$\operatorname{SNS}(N Y), \operatorname{SEW}(N X), X U(N X), Y V(N Y), R(N Y), R V(N Y), R C V(N Y), \stackrel{1}{1}$ NDCOS

$1 /$ IPOL/FXP (NX), FXM (NX), FYP (NY), FYM(NY)

1/FLUPR/URFVIS, VISCOS, DENSIT, PRANDT, DEN (NX, NY), VIS (NX, NY)

1/KASE TI/UIN, TEIN, EDIN, FLOWIN, ALAMDA,

RSMALL , RLARGE , AL1, AL2, JSTEP, ISTEP, JSTP1, JSTM1, ISTP1, ISTM1

$1 /$ TURB / GEN (NX,NY) , CD, CMU, C1, C2, CAPPA, ELOG, PRED, PRTE, PRM

$1 / \mathrm{COEF} / \mathrm{AP}(\mathrm{NX}, \mathrm{NY}), \mathrm{AN}(\mathrm{NX}, \mathrm{NY}), \mathrm{AS}(\mathrm{NX}, \mathrm{NY}), \mathrm{AE}(\mathrm{NX}, \mathrm{NY}), \mathrm{AW}(\mathrm{NX}, \mathrm{NY}), \mathrm{SU}(\mathrm{NX}, \mathrm{NY})$, $\operatorname{ISP}(\mathrm{NX}, \mathrm{NY})$

C

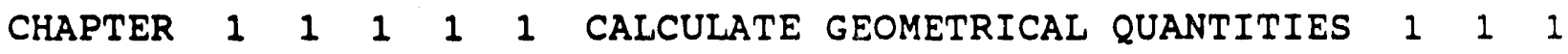
11

C

DO $100 \mathrm{~J}=1, \mathrm{NJ}$

$R(J)=Y(J)$

100 IF (INDCOS.EQ.1) R(J) $=1.0$

$\operatorname{DXPW}(1)=0.0$

$\operatorname{DXEP}(N I)=0.0$ 
DO $101 \mathrm{I}=1$, NIMI

$\operatorname{DXEP}(I)=X(I+1)-X(I)$

$101 \operatorname{DXPW}(I+1)=\operatorname{DXEP}(I)$

$\operatorname{DYPS}(1)=0.0$

$\operatorname{DYNP}(\mathrm{NJ})=0.0$

DO $102 \mathrm{~J}=1$, NJM1

$\operatorname{DYNP}(J)=Y(J+1)-Y(J)$

$102 \operatorname{DYPS}(J+1)=\operatorname{DYNP}(J)$

$\operatorname{SEW}(1)=0.0$

$\operatorname{SEW}(N I)=0.0$

DO $103 I=2$, NIMI

$103 \mathrm{SEW}(I)=0.5 *(\operatorname{DXEP}(I)+\operatorname{DXPW}(I))$

$\operatorname{SNS}(1)=0.0$

$\operatorname{SNS}(N J)=0.0$

DO $104 \mathrm{~J}=2$, NJM1

$104 \operatorname{SNS}(J)=0.5 *(\operatorname{DYNP}(J)+\operatorname{DYPS}(J))$

$X U(1)=0.0$

DO $105 \mathrm{I}=2, \mathrm{NI}$

$105 X U(I)=0.5 *(X(I)+X(I-1))$

$\operatorname{DXPWU}(1)=0.0$

$\operatorname{DXPWU}(2)=0.0$

$\operatorname{DXEPU}(1)=0.0$

$\operatorname{DXEPU}(N I)=0.0$

DO $106 I=2$, NIMI

$\operatorname{DXEPU}(I)=X U(I+1)-X U(I)$

$106 \operatorname{DXPWU}(I+1)=\operatorname{DXEPU}(I)$ 


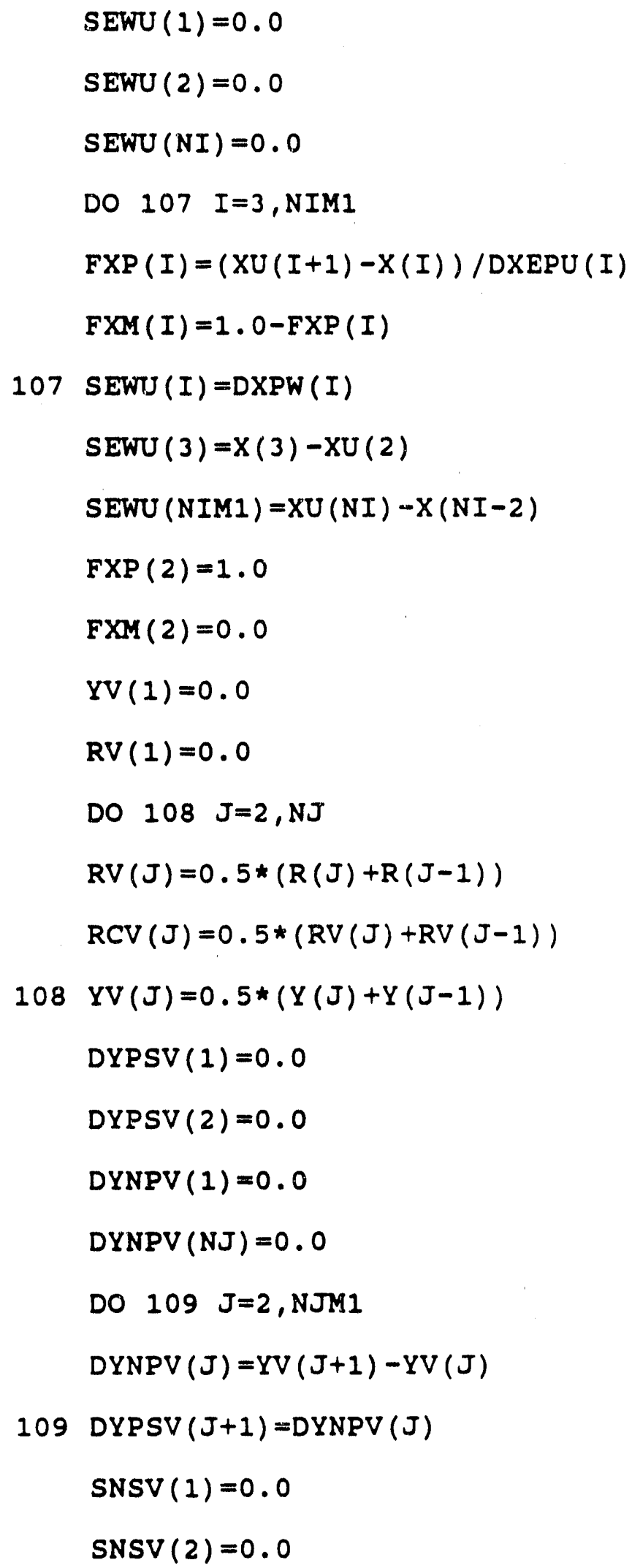




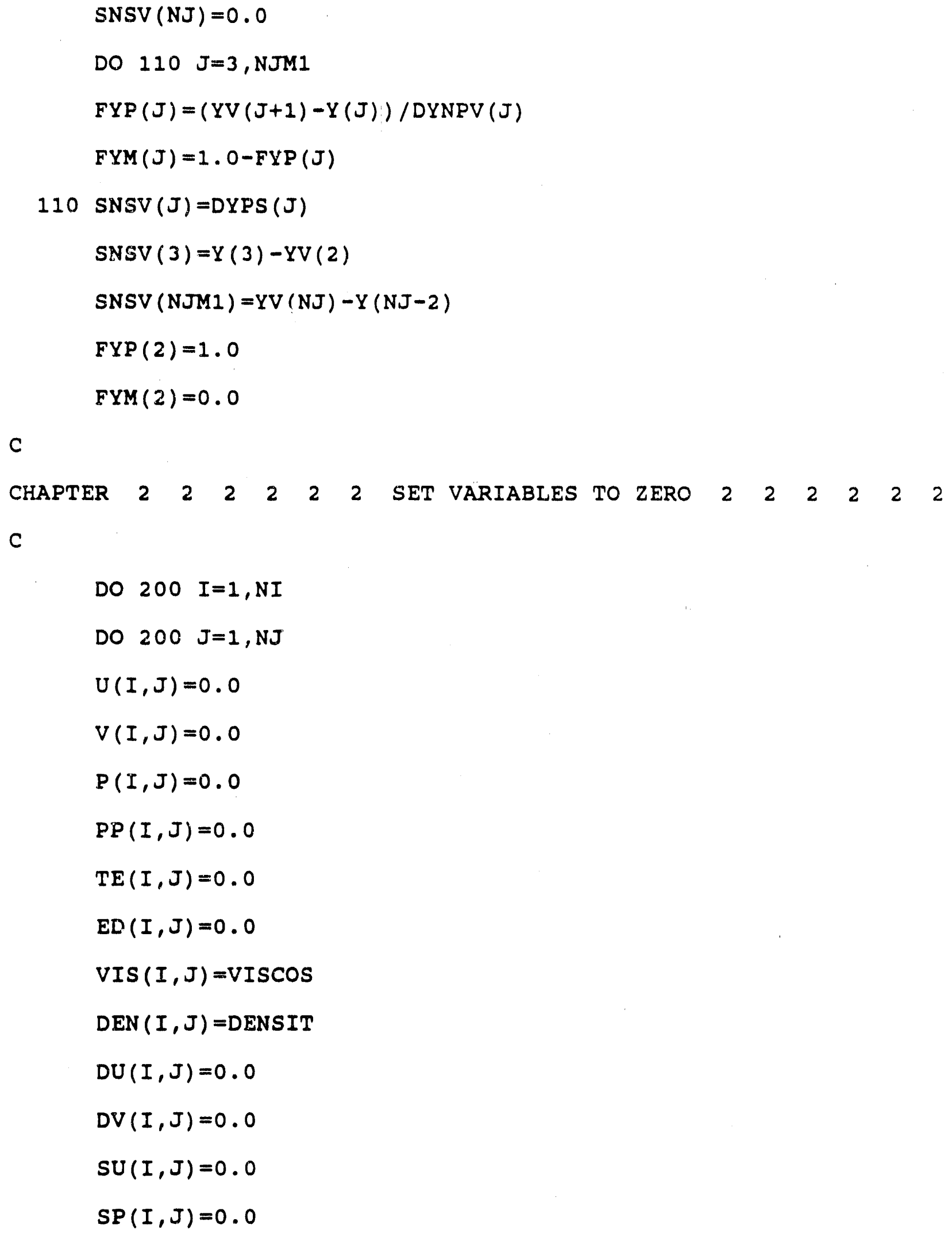


200 CONTINUE

RETURN

END

BLOCK DATA

PARAMETER $(\mathrm{NX}=50)$

PARAMETER $(N Y=26)$

COMMON/GEOM/X (NX), Y (NY), DXEP (NX), DXPW (NX), DYNP (NY) ,DYPS (NY) ,

$\operatorname{SNS}(N Y), S E W(N X), X U(N X), Y V(N Y), R(N Y), R V(N Y), R C V(N Y), \stackrel{\text { I }}{\text { NDCOS }}$

\section{d a $t$}

$(y(j), j=1, n y) /-0.5,0.5,1.5,2.5,3.5,5.5,8.5,11.5,15.5,19.5$,

$a$

$24.5,29.5,34.5,39.5,44.5,49.5,54.5,58.5,62.5$,

2

$65.5,68.5,70.5,71.5,72.5,73.5,74.5 /$

END

SUBROUTINE CALCV

C

$\begin{array}{llllllllllllllll}\text { CHAPTER } & 0 & 0 & 0 & 0 & 0 & 0 & 0 & 0 & \text { FRELIMINARIES } & 0 & 0 & 0 & 0 & 0 & 0\end{array}$ 00

C

PARAMETER $(\mathrm{NX}=50)$

PARAMETER $(N Y=26)$

COMMON

1/VVEL/RESORV, NSWFV, URFV, DYNPV (NY) , DYPSV (NY) , SNSV (NY)

1/PCOR/RESORM, NSWPP, URFP, DU (NX, NY) , DV (NX, NY) , IPREF, JPREF

$1 / V A R / U(N X, N Y), V(N X, N Y), P(N X, N Y), P P(N X, N Y), T E(N X, N Y), E D(N X, N Y)$

* $\quad 1 / A L L / I T, J T, N I, N J, N I M 1, N J M 1$, GREAT

1/all/it,jt,ni,nj,niml,njm1, great,small

$1 /$ GEOM/X(NX), Y (NY) , DXEP(NX), DXPW (NX), DYNP (NY), DYPS (NY) ,

$\operatorname{SNS}(N Y), S E W(N X), X U(N X), Y V(N Y), R(N Y), R V(N Y), R C V(N Y), \stackrel{1}{I N D C O S}$ 
$1 /$ IPOL/FXP (NX) , FXM (NX), FYP (NY), FYM(NY)

1/FLU?R/URFVIS, VISCOS, DENSIT, PRANDT, DEN (NX, NY), VIS (NX, NY)

$1 / \mathrm{COEF} / \mathrm{AP}(\mathrm{NX}, \mathrm{NY}), \mathrm{AN}(\mathrm{NX}, \mathrm{NY}), \mathrm{AS}(\mathrm{NX}, \mathrm{NY}), \mathrm{AE}(\mathrm{NX}, \mathrm{NY}), \mathrm{AW}(\mathrm{NX}, \mathrm{NY}), \mathrm{SU}(\mathrm{NX}, \mathrm{NY})$,

$1 \quad \mathrm{SP}(\mathrm{NX}, \mathrm{NY})$

1/KASE T1/UIN, TEIN, EDIN, FLOWIN, ALAMDA,

RSMALL , RLARGE , AL1, AL2, JSTEP, ISTEP, JSTP 1, JSTM1, ISTP1, ISTM1

C

$\begin{array}{lllllllllllllll}\text { CHAPTER } & 1 & 1 & 1 & 1 & 1 & 1 & \text { ASSEMBLY OF COEFFICIENTS } & 1 & 1 & 1 & 1 & 1\end{array}$ 11

C

DO $100 \quad I=2, N I M I$

DO $101 \mathrm{~J}=3$, NJM1

C-----COMPUTE AREAS AND VOLUME

$\operatorname{AREAS}=\mathrm{R}(\mathrm{J}-1) \star \mathrm{SEW}(I)$

$\operatorname{AREAN}=R(J) * S E W(I)$

AREAEW $=$ RV $(J) \star S N S V(J)$

$\operatorname{VOL}=R V(J) \star \operatorname{SEW}(I) \star \operatorname{SNSV}(J)$

C----CALCULATE CONVECTION COEFFICIENTS

$G N=0.5 *(D E N(I, J+1)+D E N(I, J)) * V(I, J+1)$

$G P=0.5 *(D E N(I, J)+D E N(I, J-1)) * V(I, J)$

$G S=0.5 *(D E N(I, J-1)+D E N(I, J-2)) * V(I, J-1)$

$G W=0.5 *(D E N(I-1, J)+D E N(I, J)) \star U(I, J)$

$G E=0.5 *(D E N(I, J)+D E N(I+1, J)) \star U(I+1, J)$

$G S W=0.5 *(D E N(I-1, J-1)+D E N(I, J-1)) * U(I, J-1)$

$\operatorname{GSE}=0.5 *(\operatorname{DEN}(I, J-1)+\operatorname{DEN}(I+1, J-1)) \star U(I+1, J-1)$

$C S=(G P \star F Y M(J-1)+G S * F Y P(J-1)) \star A R E A S$

$C N=(G N \star F Y M(J)+G P \star F Y P(J)) \star A R E A N$ 
$C E=0.5 *(G E+G S E) \star A R E A E W$

$C W=0.5 *(G W+G S W) * A R E A E W$

C

---CALCULATE DIFFUSION COEFFICIENTS

$\operatorname{VISW}=0.25 *(\operatorname{VIS}(I-1, J-1)+\operatorname{VIS}(I, J-1)+\operatorname{VIS}(I-1, J)+\operatorname{VIS}(I, J))$

$\operatorname{VISE}=0.25 *(\operatorname{VIS}(I, J)+\operatorname{VIS}(I+1, J)+\operatorname{VIS}(I, J-1)+\operatorname{VIS}(I+1, J-1))$

$D N=V I S(I, J) * A R E A N / D Y N P V(J)$

$D S=V I S(I, J-1) \star A R E A S / D Y P S V(J)$

$D E=V I S E \star A R E A E W / D X E P(I)$

$D W=V I S W * A R E A E W / D X P W(I)$

C----CALCULATE COEFFICIENTS OF SOURCE TERMS

$S M P=C N-C S+C E-C W$

$C P=A M A X 1(0.0, S M P)$

$\mathrm{CPO}=\mathrm{CP}$

C----ASSEMBLE MAIN COEFFICIENTS

$\operatorname{AN}(I, J)=\operatorname{AMAX} 1(\operatorname{ABS}(0.5 * C N), D N)-0.5 * C N$

$\operatorname{AS}(I, J)=\operatorname{AMAX} 1(\operatorname{ABS}(0.5 \star C S), D S)+0.5 \star C S$

$\operatorname{AE}(I, J)=\operatorname{AMAX} 1(\operatorname{ABS}(0.5 * C E), D E)-0.5 * C E$

$\operatorname{AW}(I, J)=\operatorname{AMAX} 1(\operatorname{ABS}(0.5 * C W), D W)+0.5 * C W$

$\operatorname{DV}(I, J)=0.5 *($ AREAN + AREAS $)$

$\operatorname{SU}(I, J)=C P O * V(I, J)+D V(I, J) *(P(I, J-1)-P(I, J))$

$\operatorname{SP}(I, J)=-C P$

C IF (INDCOS.EQ.2) $\operatorname{SP}(I, J)=\operatorname{SP}(I, J)-V I S(I, J) \star V O L / R V(J) \star \star 2$

$\operatorname{IF}(\operatorname{INDCOS} . \mathrm{EQ} .2) \quad \operatorname{SP}(I, J)=\operatorname{SP}(I, J)-2.0 * \operatorname{VIS}(I, J) \star \operatorname{VOL} / \mathrm{RV}(J) \star \star 2$

$\operatorname{DUDYP}=(U(I+1, J)-U(I+1, J-1)) / \operatorname{DYPS}(J)$

$\operatorname{GAMM}=0.25 *(\operatorname{VIS}(I-1, J-1)+\operatorname{VIS}(I, J-1)+\operatorname{VIS}(I-1, J)+\operatorname{VIS}(I, J))$

$\operatorname{GAMP}=0.25 *(\operatorname{VIS}(I, J)+\operatorname{VIS}(I+1, J)+\operatorname{VIS}(I, J-1)+\operatorname{VIS}(I+1, J-1))$ 


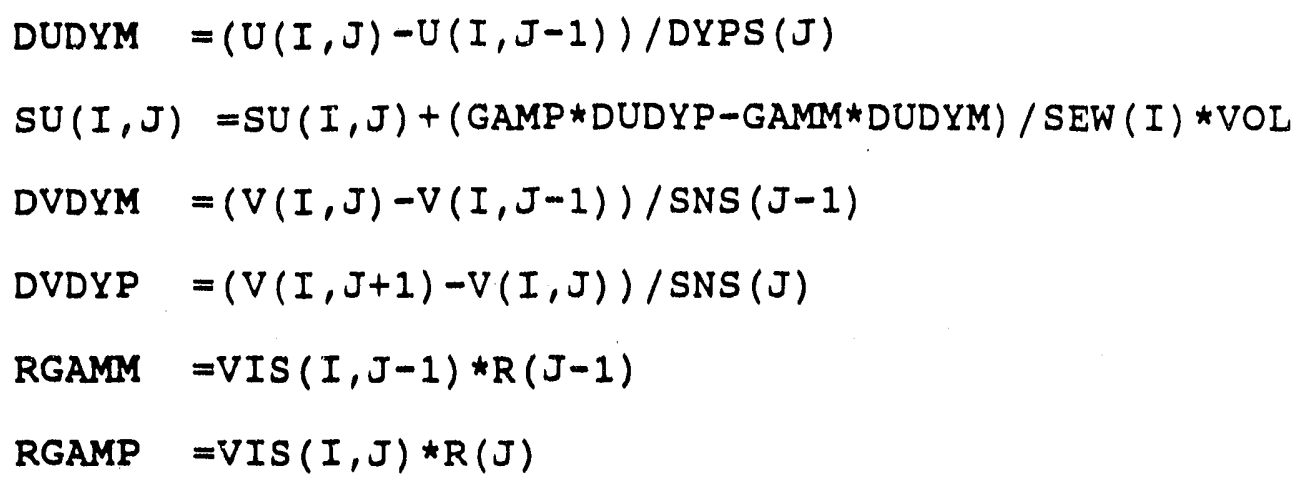




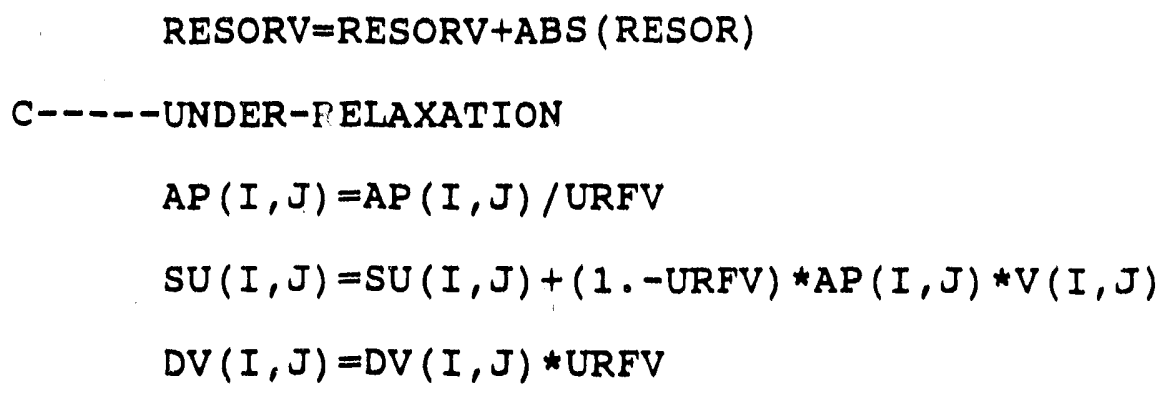


$1 /$ GEOM/X (NX), Y (NY) , DXEP (NX), DXPW (NX), DYNP (NY), DYPS (NY) ,

$\operatorname{SNS}(N Y), \operatorname{SEW}(N X), X U(N X), Y V(N Y), R(N Y), R V(N Y), R C V(N Y), \stackrel{1}{I} \operatorname{INDCOS}$

$1 /$ IPOL/FXP (NX), FXM (NX), FYP(NY), FYM(NY)

1/FLUPR/URFVIS, VISCOS, DENSIT, PRANDT, DEN (NX, NY) , VIS (NX, NY)

$1 / C O E F / A P(N X, N Y), A N(N X, N Y), A S(N X, N Y), A E(N X, N Y), A W(N X, N Y), S U(N X, N Y)$,

$1 \quad S P(N X, N Y)$

1/KASE T1/UIN, TEIN, EDIN, FLOWIN, ALAMDA,

RSMALL, RLARGE , AL1, AL2 , JSTEP , ISTEP, JSTP 1, JSTM1, ISTP1, ISTM1

C

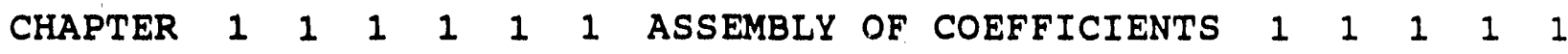
11

C

DO $100 I=3$, NIM 1

DO $101 \mathrm{~J}=2$, NJM1

C-----COMPUTE AREAS AND VOLUME

$\operatorname{AREAS}=\mathrm{RV}(\mathrm{J}) \star S E W U(I)$

$\operatorname{AREAN}=\mathrm{RV}(\mathrm{J}+1) * \operatorname{SEWU}(I)$

$\operatorname{AREAEW}=\operatorname{RCV}(J+1) \star \operatorname{SNS}(J)$

$\operatorname{VOL}=\operatorname{RCV}(J+1) * \operatorname{SEWU}(I) * \operatorname{SNS}(J)$

C----CALCULATE CONVECTION COEFFICIENTS

$\operatorname{GNW}=0.5 *(\operatorname{DEN}(I-1, J)+\operatorname{DEN}(I-1, J+1)) * V(I-1, J+1)$

$G N=0.5 *(D E N(I, J+1)+D E N(I, J)) \star V(I, J+1)$

$\operatorname{GSW}=0.5 *(\operatorname{DEN}(I-1, J)+\operatorname{DEN}(I-1, J-1)) * V(I-1, J)$

$G S=0.5 *(D E N(I, J-1)+D E N(I, J)) \star V(I, J)$

$G W=0.5 *(D E N(I-2, J)+D E N(I-1, J)) \star U(I-1, J)$

$\operatorname{GP}=0.5 *(\operatorname{DEN}(I-1, J)+\operatorname{DEN}(I, J)) * U(I, J)$

$G E=0.5 *(D E N(I, J)+D E N(I+1, J)) * U(I+1, J)$ 


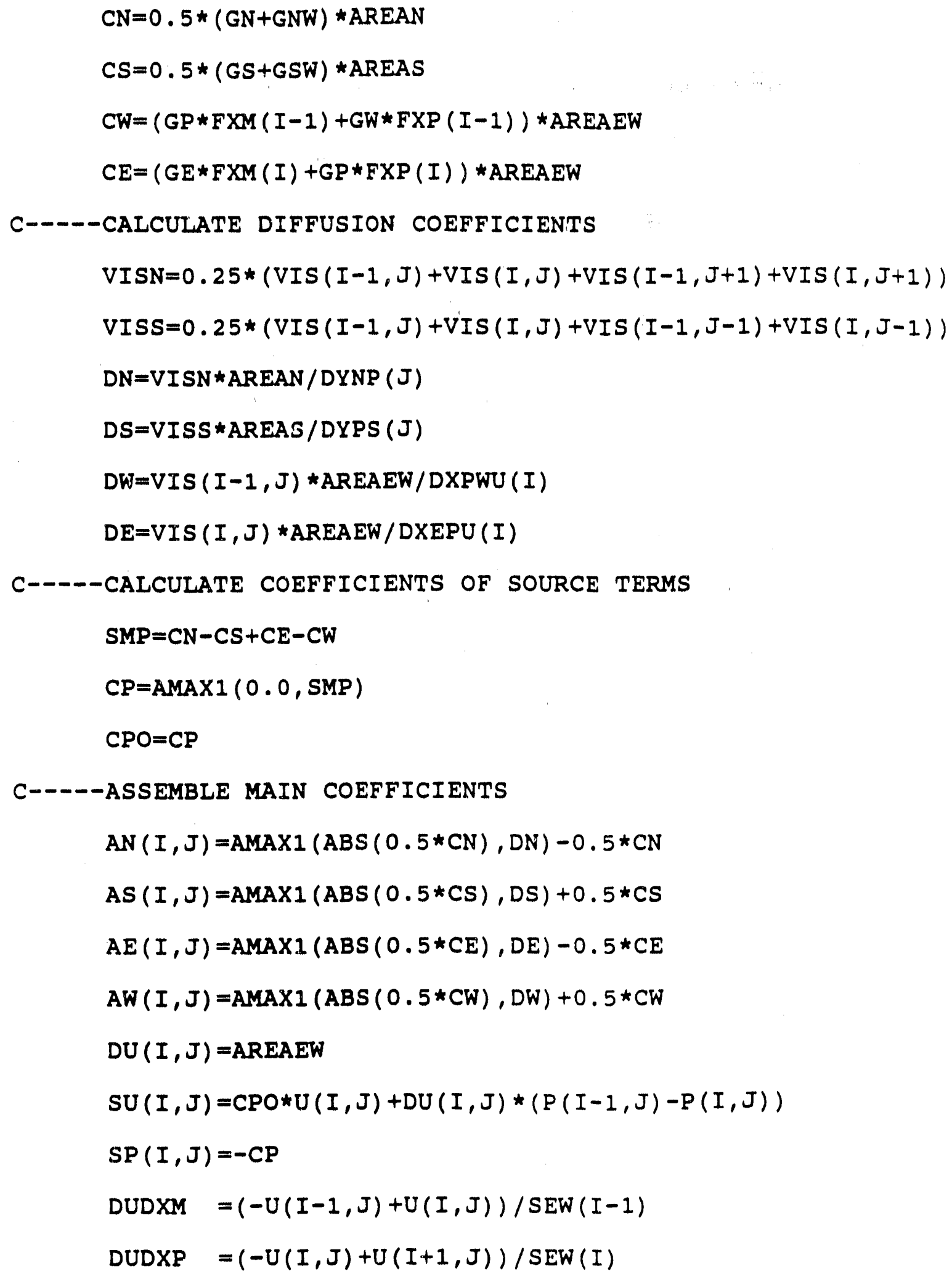




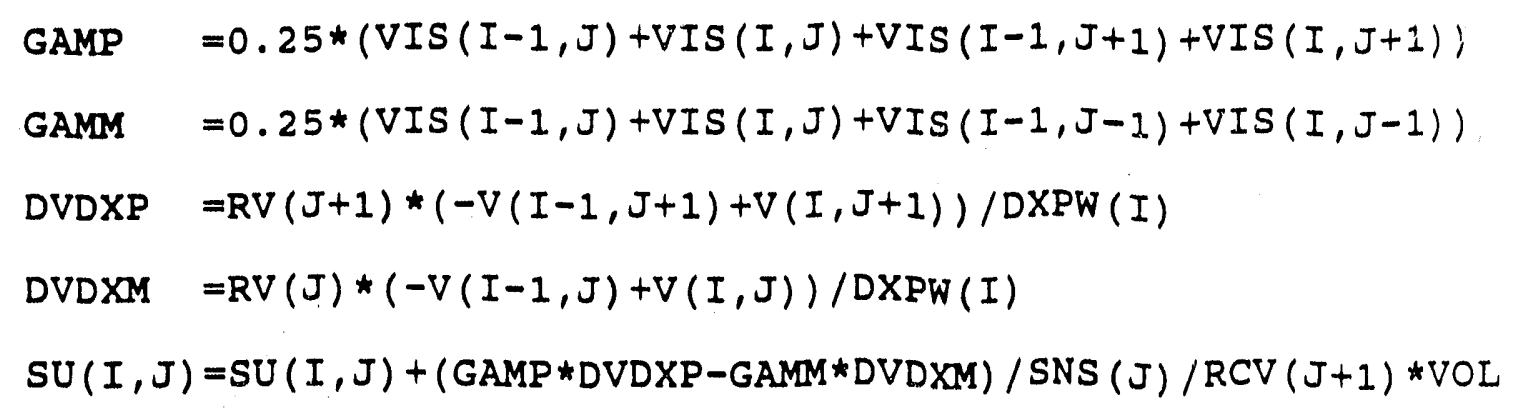

101 CONTINUE

100 CONTINUE

c

$\begin{array}{llllllllllllll}\text { CHAPTER } & 2 & 2 & 2 & 2 & 2 & 2 & 2 & \text { PROBLEM MODIFICATIONS } & 2 & 2 & 2 & 2 & 2\end{array}$ 22

C

CALL MODU

C

CHAPTER 3 FINAL COEFF. ASSEMBLY AND RESIDUAL SOURCE CALCULATION 33

C

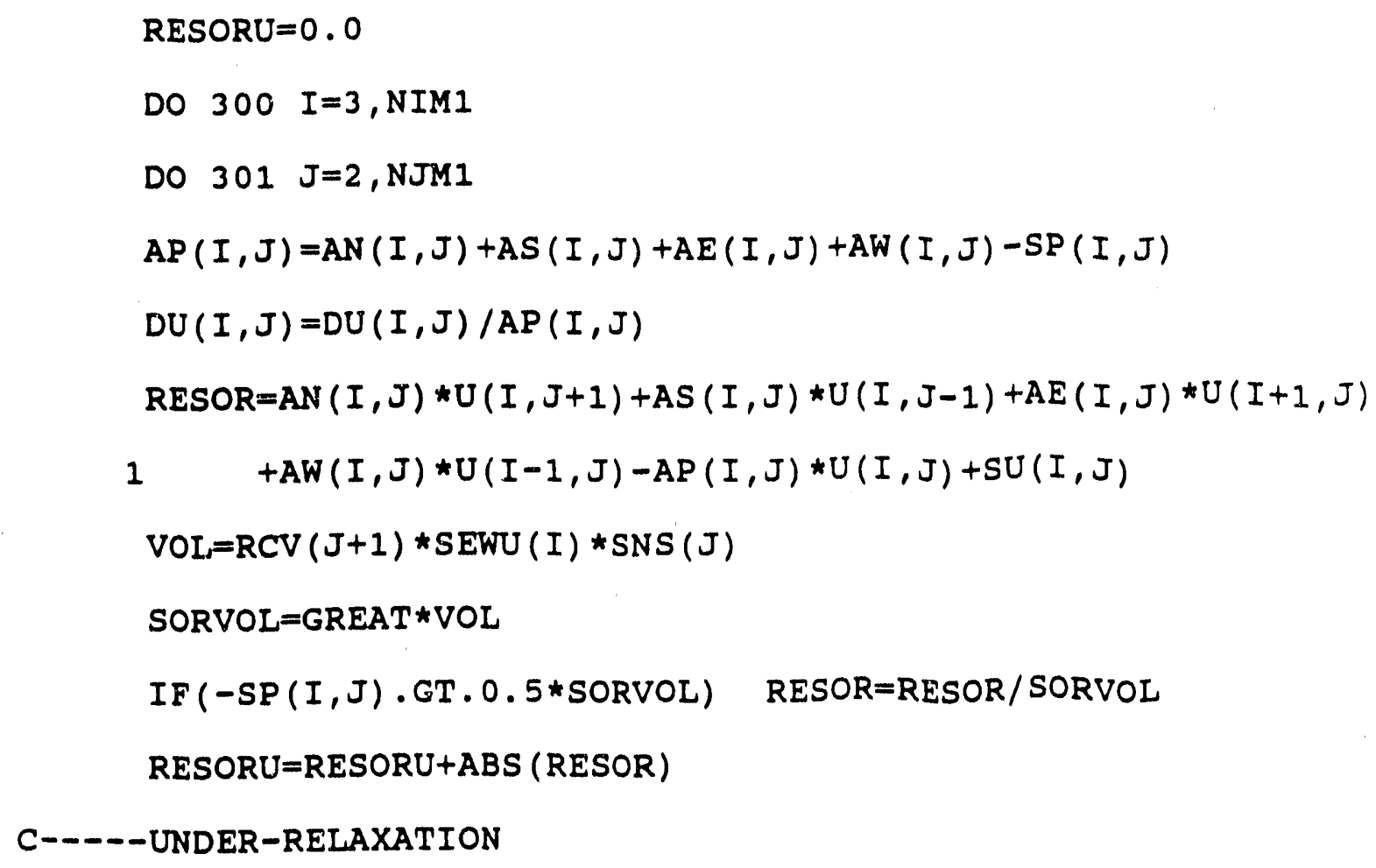




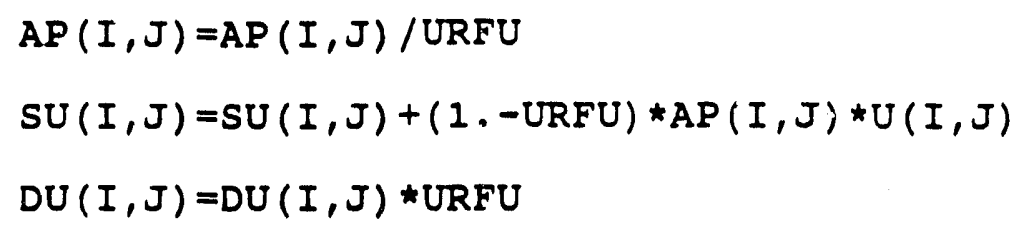

301 CONTINUE

300 CONTINUE

C

$\begin{array}{lllllllllll}\text { CHAPTER } & 4 & 4 & 4 & \text { SOLUTION OF DIFFERENCE EQUATION } & 4 & 4 & 4 & 4 & 4\end{array}$ $4 \quad 4$

DO $400 \mathrm{~N}=1$, NSWPU

400 CALL $\operatorname{LISOLV}(3,2, N I, N J, I T, J T, U)$

RETURN

END

SUBROUTINE CALCTE

C

$\begin{array}{llllllllllllllll}\text { CHAPTER } & 0 & 0 & 0 & 0 & 0 & 0 & 0 & \text { PRELIMINARIES } & 0 & 0 & 0 & 0 & 0 & 0 & 0\end{array}$ C

PARAMETER $(\mathrm{NX}=50)$

PARAMETER $(N Y=26)$

COMMON

1/TEN/RESORK, NSWPK, URFK

$1 / V A R / U(N X, N Y), V(N X, N Y), P(N X, N Y), P P(N X, N Y), T E(N X, N Y), E D(N X, N Y)$

* $\quad 1 / A L L / I T, J T, N I, N J, N I M 1, N J M 1$, GREAT

1/all/it,jt, ni, nj, niml, njm1, great, small

$1 / G E O M / X(N X), Y(N Y), D X E P(N X), D X P W(N X), D Y N P(N Y), D Y P S(N Y)$ ，

SNS (NY), SEW (NX), XU (NX; , YV (NY), R(NY), RV (NY), RCV (NY), INDCOS

$1 /$ IPOL/FXP (NX), FXM (NX), FYP(NY), FYM (NY) 
1/FLUPR/URFVIS, VISCOS, DENSIT, PRANDT , DEN (NX, NY) , VIS (NX, NY)

$1 / C O E F / A P(N X, N Y), A N(N X, N Y), A S(N X, N Y), A E(N X, N Y), A W(N X, N Y), S U(N X, N Y)$, $1 \quad \mathrm{SP}(\mathrm{NX}, \mathrm{NY})$

$1 /$ TURB / GEN (NX, NY) , CD , CMU , C1, C2, CAPPA, ELOG, PRED , PRTE , PRM

1 / WALLF / YPLUSN (NX) , XPLUSW (NY), TAUN (NX), TAUW (NY)

1/KASE T1/UIN, TEIN, EDIN, FLOWIN, ALAMDA,

2

RSMALL, RLARGE, AL1, AL2, JSTEP, ISTEP, JSTP1, JSTM1 , ISTP1, ISTM1

$1 / \operatorname{SUSP} / \operatorname{SUKD}(N X, N Y), \operatorname{SPKD}(N X, N Y)$

C

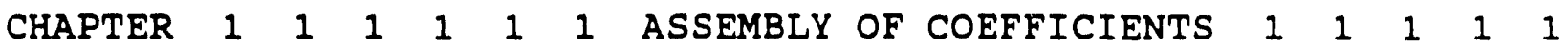
1

C

PRTE $=1.0$

DO $100 \quad I=2$, NIMI

DO $101 \mathrm{~J}=2$, NJM1

C-----COMPUTE AREAS AND VOLUME

$\operatorname{AREAN}=\operatorname{RV}(J+1) \star \operatorname{SEW}(I)$

$\operatorname{AREAS}=R V(J) \star S E W(I)$

$\operatorname{AREAEW}=\operatorname{RCV}(T+1) * \operatorname{SNS}(\mathrm{J})$

$\operatorname{VOL}=\operatorname{RCV}(J+1) * \operatorname{SNS}(J)$ *SEW $(I)$

C----CALCULATE CONVECTION COEFFICIENTS

$G N=0.5 \star(D E N(I, J)+D E N(I, J+1)) \star V(I, J+1)$

$G S=0.5 *(D E N(I, J)+D E N(I, J-1)) \star V(I, J)$

$G E=0.5 *(D E N(I, J)+D E N(I+1, J)) * U(I+1, J)$

$G W=0.5 *(D E N(I, J)+D E N(I-1, J)) \star U(I, J)$

$\mathrm{CN}=\mathrm{GN} \star A R E A N$

$C S=G S \star A R E A S$ 


\section{$C E=G E \star A R E A E W$}

$\mathrm{CW}=\mathrm{GW} \star \mathrm{AREAEW}$

C----CALCULATE DIFFUSION COEFFICIENTS

$\operatorname{GAMN}=0.5 *(\operatorname{VIS}(I, J)+\operatorname{VIS}(I, J+1)) /$ PRTE

GAMS $=0.5 *(V I S(I, J)+\operatorname{VIS}(I, J-1)) /$ PRTE

GAME $=0.5 *(V I S(I, J)+\operatorname{VIS}(I+1, J)) /$ PRTE

GAMW $=0.5$ * $(\operatorname{VIS}(I, J)+\operatorname{VIS}(I-I, J)) /$ PRTE

$D N=G A M N * A R E A N / D Y N P(J)$

$D S=G A M S * A R E A S / D Y P S(J)$

$D E=G A M E \star A R E A E W / D X E P(I)$

$D W=G A M W \star A R E A E W / D X P W(I)$

C-----SOURCE TERMS

$S M P=C N-C S+C E-C W$

$C P=A M A X 1(0.0, S M P)$

$\mathrm{CPO}=\mathrm{CP}$

$\operatorname{DUDX}=(U(I+1, J)-U(I, J)) / S E W(I)$

$\operatorname{DVDY}=(V(I, J+1)-V(I, J)) / \operatorname{SNS}(J)$

$\operatorname{DUDY}=((U(I, J)+U(I+1, J)+U(I, J+1)+U(I+1, J+1)) / 4 .-(U(I, J)+U(I+1, J)+$ $I U(I, J-1)+U(I+1, J-1)) / 4$.)/SNS (J)

$D V D X=((V(I, J)+V(I, J+1)+V(I+1, J)+V(I+1, J+1)) / 4 .-(V(I, J)+V(I, J+1)+V($ $I I-1, J)+V(I-1, J+1)) / 4.) / S E W(I)$

$\operatorname{GEN}(I, J)=(2 . \star(D U D X \star \star 2+D V D Y \star \star 2)+(D U D Y+D V D X) \star \star 2) \star V I S(I, J)$

$\operatorname{IF}(R V(J) \cdot E Q \cdot 0.0)$ GOTO 77

$\operatorname{VDR}=V(I, J) / R V(J)$

77 IF (J.EQ.2) $\quad \mathrm{VDR}=0.0$

$\operatorname{IF}(\operatorname{INDCOS} . \mathrm{EQ} .2) \quad \operatorname{GEN}(I, J)=\mathrm{GEN}(I, J)+\operatorname{VIS}(I, J) * 0.5 *(V D R+V(I, J+1) /$ 
$1 \quad \operatorname{RV}(J+1)) \star \star 2$

C----ASSEMBLE MAIN COEFFICIENTS

$$
\begin{aligned}
& \operatorname{AN}(I, J)=\operatorname{AMAX} 1(\operatorname{ABS}(0.5 * C N), D N)-0.5 * C N \\
& \operatorname{AS}(I, J)=\operatorname{AMAXI}(\operatorname{ABS}(0.5 * C S), D S)+0.5 * C S \\
& \operatorname{AE}(I, J)=\operatorname{AMAX} 1(\mathrm{ABS}(0.5 * C E), D E)-0.5 * C E \\
& \operatorname{AW}(I, J)=\operatorname{AMAXI}(\operatorname{ABS}(0.5 * C W), D W)+0.5 * C W \\
& \operatorname{SU}(I, J)=\operatorname{CPO} * \operatorname{TE}(I, J) \\
& \operatorname{SUKD}(I, J)=\operatorname{SU}(I, J) \\
& S U(I, J)=S U(I, J)+G E N(I, J) \text { *VOL } \\
& \operatorname{SP}(I, J)=-C P \\
& \operatorname{SPKD}(I, J)=\operatorname{SP}(I, J) \\
& \operatorname{SP}(I, J)=\operatorname{SP}(I, J)-C D * \operatorname{CMU} \star \operatorname{DEN}(I, J) \star \star 2 \star T E(I, J) \star V O I / V I S(I, J)
\end{aligned}
$$

101 CONTINUE

100 CONTINUE

C

$\begin{array}{llllllllllllll}\text { CHAPTER } & 2 & 2 & 2 & 2 & 2 & 2 & \text { PROBLEM MODIFICATIONS } & 2 & 2 & 2 & 2 & 2 & 2\end{array}$ C

CALL MODTE

C

CHAPTER 3 FINAL COEFFICIENT ASSEMBLY AND RESIDUAL SOURCE CALCUIATION 3

C

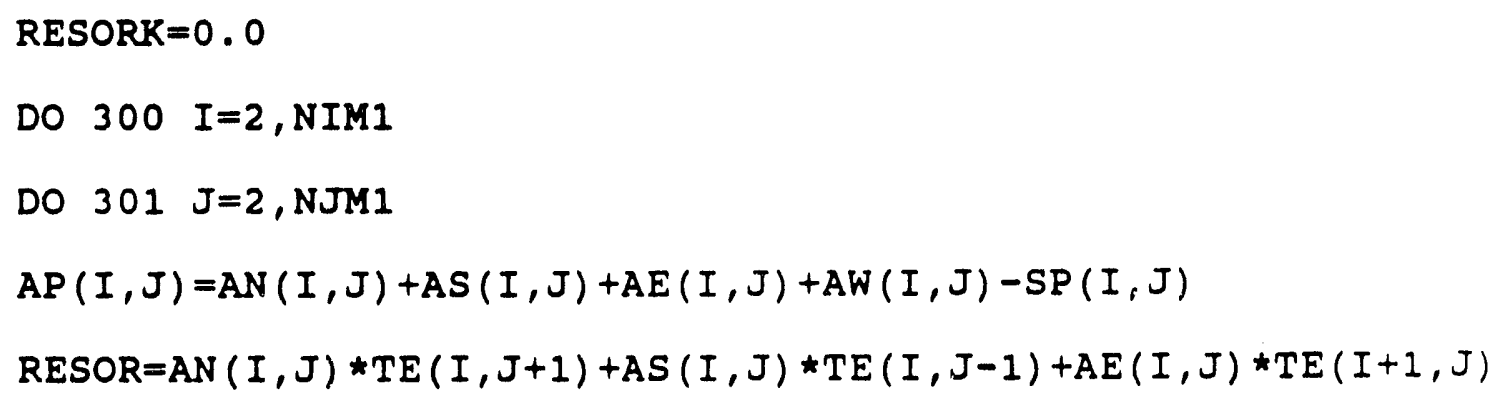




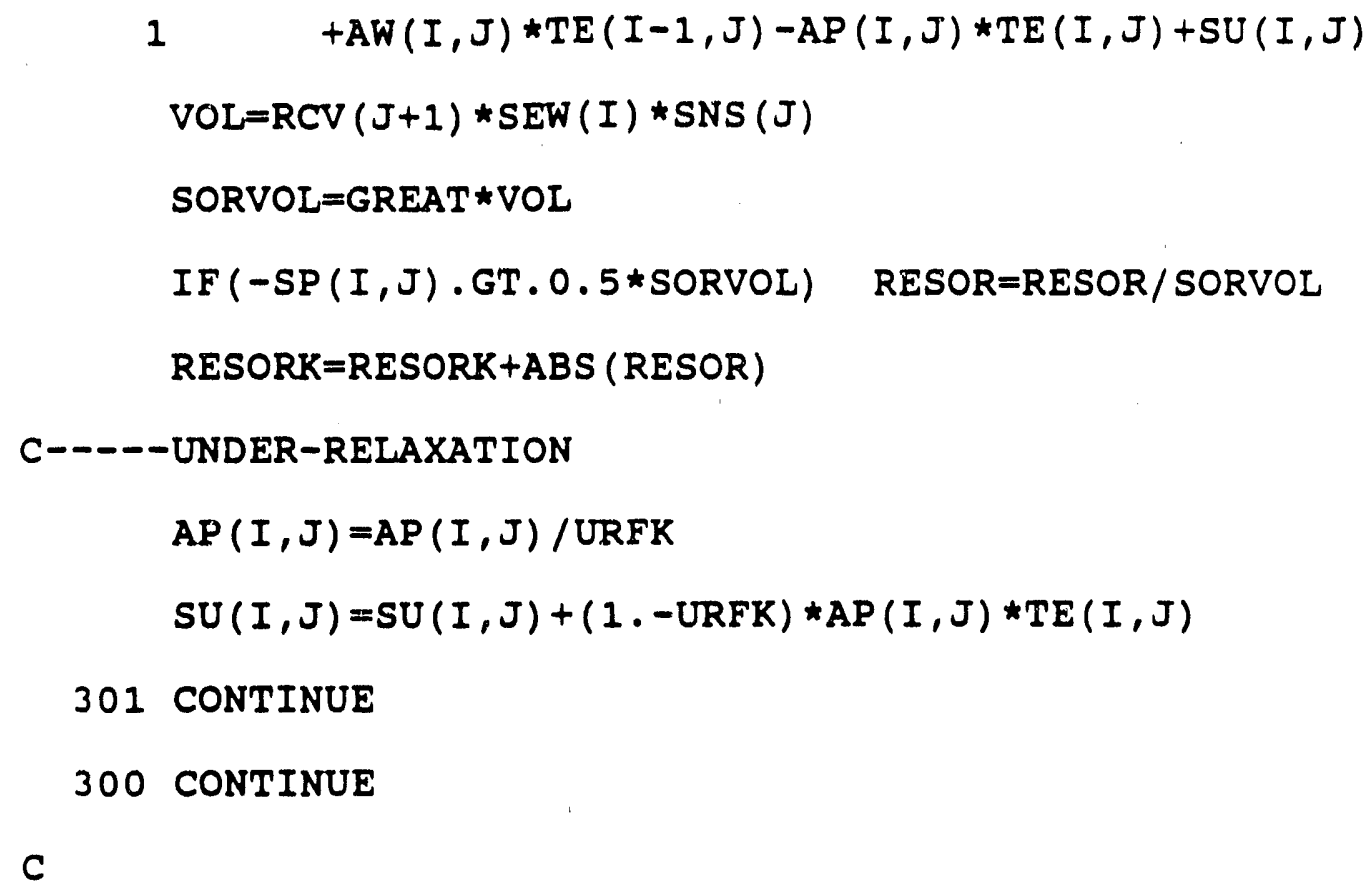


* $\quad 1 /$ ALL/IT, JT, NI, NJ , NIM1, NJM1, GREAT

1/all/it,jt,ni,nj,nim1, njm1, great,small

$1 / G E O M / X(N X), Y(N Y), D X E P(N X), D X P W(N X), D Y N P(N Y), D Y P S(N Y)$ ，

$\operatorname{SNS}(N Y), S E W(N X), X U(N X), Y V(N Y), R(N Y), R V(N Y), R C V(N Y), \stackrel{1}{\operatorname{INDCOS}}$

$1 /$ IPOL/FXP (NX) , FXM (NX) , FYP (NY) , FYM (NY)

1/FLUPR/URFVIS, VISCOS, DENSIT, PRANDT, DEN (NX, NY), VIS (NX, NY)

$1 / C O E F / A P(N X, N Y), A N(N X, N Y), A S(N X, N Y), A E(N X, N Y), A W(N X, N Y), S U(N X, N Y)$,

$1 \quad S P(N X, N Y)$

1/KASE T1/UIN, TEIN, EDIN, FLOWIN, ALAMDA,

RSMALL , RLARGE , AL1, AL2, JSTEP , ISTEP, JSTP1, JSTM1, ISTP1, ISTM1

RESORM $=0.0$

C

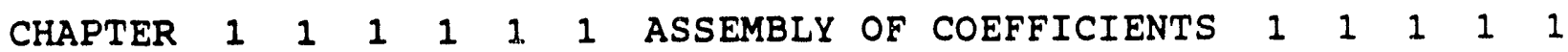
11

C

DO $100 \quad I=2, N I M 1$

DO $101 \mathrm{~J}=2$, NJM1

C-----COMPUTE AREAS AND VOLUME

AREAS $=R V(J) \star S E W(I)$

$\operatorname{AREAN}=\mathrm{RV}(\mathrm{J}+1) \star \mathrm{SEW}(\mathrm{I})$

$\operatorname{AREAEW}=\operatorname{RCV}(J+1) * \operatorname{SNS}(\mathrm{J})$

$\operatorname{VOL}=\operatorname{RCV}(J+1)$ *SNS $(J)$ *SEW $(I)$

C----CALCULATE COEFFICIENTS

$\operatorname{DENN}=0.5 *(\operatorname{DEN}(I, J)+\operatorname{DEN}(I, J+1))$

$\operatorname{DENS}=0.5 *(\operatorname{DEN}(I, J)+\operatorname{DEN}(I, J-1))$

$\operatorname{DENE}=0.5$ * $(\operatorname{DEN}(I, J)+\operatorname{DEN}(I+1, J))$

$\operatorname{DENW}=0.5 *(\operatorname{DEN}(I-1, J)+\operatorname{DEN}(I, J))$ 


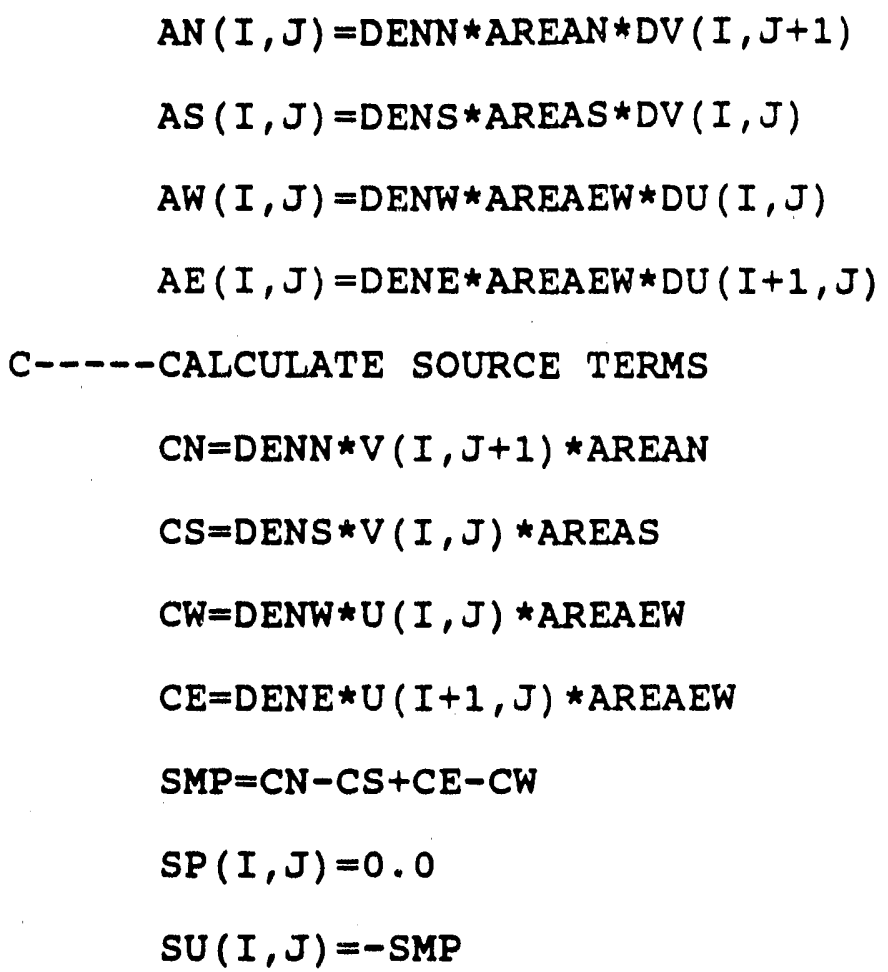


300 CONTINUE

C

$\begin{array}{llllllllllll}\text { CHAPTER } & 4 & 4 & 4 & 4 & 4 & \text { SOLUTION OF } & \text { DIFFERENCE } & \text { EQUATIONS } & 4 & 4 & 4\end{array}$ 44

C

DO $400 \mathrm{~N}=1$, NSWPP

400 CALL LISOLV $(2,2, N I, N J, I T, J T, P P)$

C

$\begin{array}{llllllllllll}\text { CHAPTER } & 5 & 5 & 5 & 5 & \text { CORRECT VELOCITIES AND PRESSURE } & 5 & 5 & 5 & 5\end{array}$ 55

C

C-----VELOCITIES

DO $500 \quad I=2$, NIM1

DO $501 \mathrm{~J}=2$, NJM1

$\operatorname{IF}(I . N E .2) \quad U(I, J)=U(I, J)+D U(I, J) *(P P(I-I, J)-P P(I, J))$

$I F(J . N E .2) \quad V(I, J)=V(I, J)+D V(I, J) *(P P(I, J-1)-P P(I, J))$

501 CONTINUE

500 CONTINUE

C-----PRESSURES (WITH PROVISION FOR UNDER-RELAXATION)

PPREF $=$ PP $($ IPREF , JPREF $)$

DO $502 \quad I=2$, NIMI

DO $503 \mathrm{~J}=2$, NJMI

$P(I, J)=P(I, J)+U R F P *(P P(I, J)-P P R E F)$

$\operatorname{PP}(I, J)=0.0$

503 CONTINUE

502 CONTINUE

RETURN

END 


\section{SUBROUTINE CALCM}

C

$\begin{array}{llllllllllllllll}\text { CHAPTER } & 0 & 0 & 0 & 0 & 0 & 0 & 0 & \text { PRELIMINARIES } & 0 & 0 & 0 & 0 & 0 & 0 & 0\end{array}$ C

PARAMETER $(\mathrm{NX}=50)$

PARAMETER (NY=26)

COMMON

1/TDIS/RESORE, NSWPD, URFE

* $\quad 1 / A L L / I T, J T, N I, N J, N I M 1, N J M 1$, GREAT

1/all/it,jt,ni,nj,niml,njml,great,small

$1 / G E O M / X(N X), Y(N Y), D X E P(N X), D X P W(N X), D Y N P(N Y), D Y P S(N Y)$,

SNS (NY), SEW (NX), XU (NX), YV (NY), R (NY), RV (NY), RCV (NY), INDCOS

$1 /$ IPOL/FXP (NX), FXM(NX) , FYP (NY) , FYM (NY)

1/FLUPR/URFVIS, VISCOS, DENSIT, PRANDT, DEN (NX, NY) , VIS (NX, NY)

$1 / C O E F / A P(N X, N Y), A N(N X, N Y), A S(N X, N Y), A E(N X, N Y), A W(N X, N Y), S U(N X, N Y)$, 1 $\mathrm{SP}(\mathrm{NX}, \mathrm{NY})$

$1 /$ TURB/GEN (NX, NY) , CD , CMU , C1, C2 , CAPPA, ELOG , PRED , PRTE, PRM

$1 /$ SUSP / SUKD (NX,NY) , SPKD (NX,NY)

$1 / V A R / U(N X, N Y), V(N X, N Y), P(N X, N Y), P P(N X, N Y), T E(N X, N Y), E D(N X, N Y)$

1/VARE / AM (NX, NY)

1/KASE T1/UIN, TEIN, EDIN, FLOWIN, ALAMDA,

2

RSMALL, RLARGE , AL1, AL2 , JSTEP, ISTEP , JSTP1, JSTM1, ISTP1, ISTM1 C

$\begin{array}{lllllllllllllll}\text { CHAPTER } & 1 & 1 & 1 & 1 & 1 & 1 & \text { ASSEMBLY OF COEFFICIENTS } & 1 & 1 & 1 & 1 & 1\end{array}$ 1

C

DO $100 \quad I=2$, NIMI 
DO $101 \mathrm{~J}=2$, NJM1

C-----COMPUTE AREAS AND VOLUME

$A R E A N=R V(J+1)$ *SEW $(I)$

$\operatorname{AREAS}=\mathrm{RV}(\mathrm{J})$ *SEW $(I)$

$\operatorname{AREAEW}=\operatorname{RCV}(J+1) * \operatorname{SNS}(J)$

$\operatorname{VOL}=\operatorname{RCV}(J+1) \star \operatorname{SNS}(J) * \operatorname{SEW}(I)$

C----CALCULATE CONVECTION COEFFICIENTS

$\operatorname{GN}=0.5 *(\operatorname{DEN}(I, J)+\operatorname{DEN}(I, J+1)) * V(I, J+1)$

$G S=0.5 *(D E N(I, J)+D E N(I, J-1)) * V(I, J)$

$G E=0.5 *(D E N(I, J)+D E N(I+1, J)) \star U(I+1, J)$

$G W=0.5 *(D E N(I, J)+\operatorname{DEN}(I-1, J)) \star U(I, J)$

$\mathrm{CN}=\mathrm{GN} * \mathrm{AREAN}$

CS $=G S * A R E A S$

$C E=G E \star A R E A E W$

$C W=G W * A R E A E W$

C----CALCULATE DIFFUSION COEFFICIENTS

$\operatorname{GAMN}=0.5 *(\operatorname{VIS}(I, J ;+\operatorname{VIS}(I, J+1)) / P R M$

GAMS $=0.5 *(\operatorname{VIS}(I, J)+\operatorname{VIS}(I, J-1)) / P R M$

$\operatorname{GAME}=0.5 *(\operatorname{VIS}(I, J)+\operatorname{VIS}(I+1, J)) / P R M$

GAMW $=0.5 *(\operatorname{VIS}(I, J)+\operatorname{VIS}(I-1, J)) / P R M$

$D N=G A M N * A R E A N / D Y N P(J)$

DS=GAMS *AREAS / DYPS $(\mathrm{J})$

$D E=G A M E \star A R E A E W / D X E P(I)$

$D W=G A M W * A R E A E W / D X P W(I)$

C-----SOURCE TERMS

$S M P=C N-C S+C E-C W$ 
$C P=A M A X 1(0.0, S M P)$

$\mathrm{CPO}=\mathrm{CP}$

C----ASSEMBLE MAIN COEFFICIENTS

$\operatorname{AN}(I, J)=\operatorname{AMAX} 1(\operatorname{ABS}(0.5 \star C N), D N)-0.5 \star C N$

$\operatorname{AS}(I, J)=\operatorname{AMAX1}(\operatorname{ABS}(0.5 * C S), D S)+0.5 * C S$

$\operatorname{AE}(I, J)=\operatorname{AMAX} 1(\operatorname{ABS}(0.5 * C E), D E)-0.5 * C E$

$\operatorname{AW}(I, J)=\operatorname{AMAX} 1(\operatorname{ABS}(0.5 * C W), D W)+0.5 * C W$

$\mathrm{SU}(I, J)=\mathrm{CPO} * \mathrm{AM}(I, J)$

$\operatorname{SP}(I, J)=-C P$

101 CONTINUE

100 CONTINUE

C

$\begin{array}{llllllllllllll}\text { CHAPTER } & 2 & 2 & 2 & 2 & 2 & 2 & \text { PROBLEM MODIFICATIONS } & 2 & 2 & 2 & 2 & 2 & 2\end{array}$ C

CALL MODT

C

CHAPTER 3 FINAL COEFFICIENT ASSEMBLY AND RESIDUAL SOURCE CALCULATION 3

C

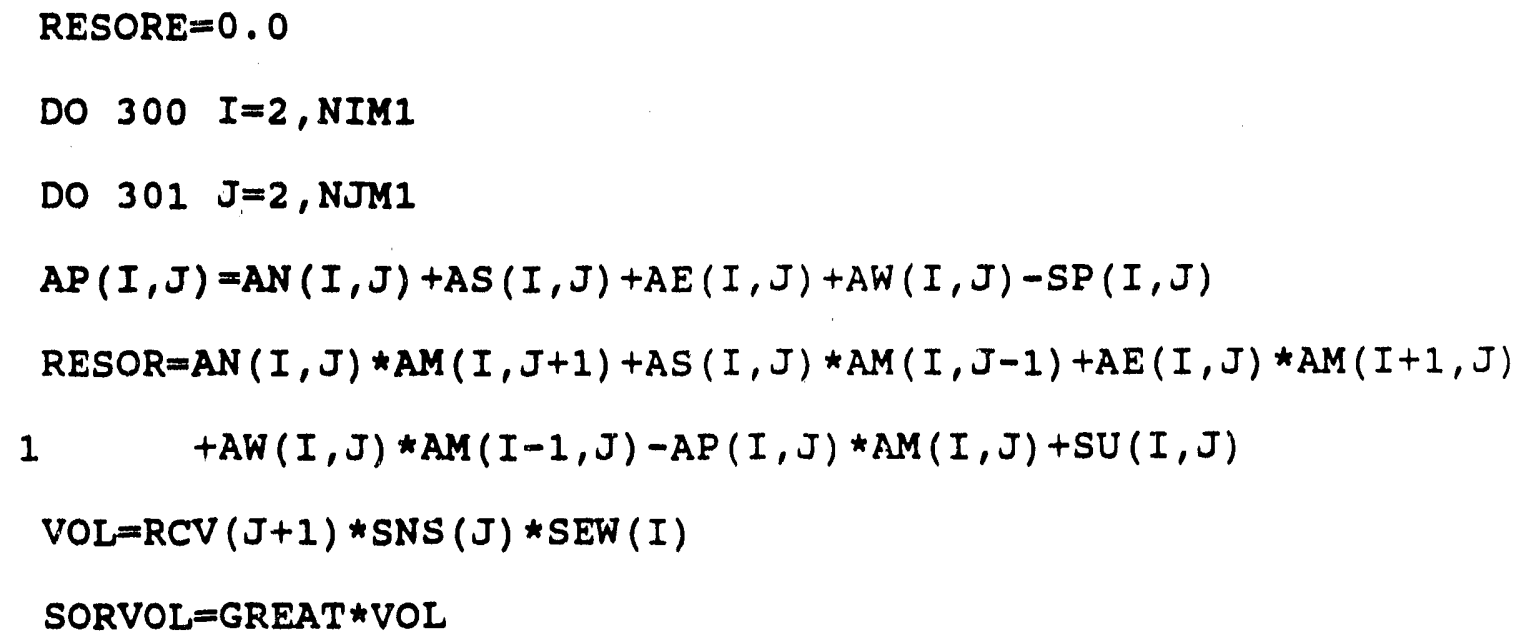


IF (-SP $(I, J) \cdot G T \cdot 0.5 *$ SORVOL) RESOR=RESOR/SORVOL

RESORE=RESORE+ABS (RESOR)

C-----UNDER-RELAXATION

$\operatorname{AP}(I, J)=\operatorname{AP}(I, J) / U R F E$

$\operatorname{SU}(I, J)=S U(I, J)+(1 .-U R F E) * A P(I, J) * A M(I, J)$

301 CONTINUE

300 CONTINUE

C

$\begin{array}{lllllllllll}\text { CHAPTER } & 4 & 4 & 4 & 4 & 4 & \text { SOLUTION OF DIFFERENCE EQUATIONS } & 4 & 4 & 4\end{array}$ 44

C

DO $400 \mathrm{~N}=1$, NEWWPD

400 CALI LISOLV $(2,2, N I, N J, I T, J T, A M)$

RETURN

END

SUBROUTINE CALCED

C

$\begin{array}{llllllllllllllll}\text { CHAPTER } & 0 & 0 & 0 & 0 & 0 & 0 & 0 & \text { PRELIMINARIES } & 0 & 0 & 0 & 0 & 0 & 0 & 0\end{array}$

C

PARAMETER $(N X=50)$

PARAMETER $(N Y=26)$

COMMON

1/TDIS/RESORE, NSWPD, URFE

* $\quad 1 / A L L / I T, J T, N I, N J, N I M 1, N J M 1$, GREAT

1/all/it,jt,ni,nj, niml,njml, great, small

1/GEOM/X(NX), Y (NY), DXEP(NX), DXPW (NX), DYNP (NY), DYPS (NY),

$\operatorname{SNS}(N Y), \operatorname{SEW}(N X), X U(N X), Y V(N Y), R(N Y), R V(N Y), R C V(N Y), \operatorname{INDCOS}$ 
1/IPOL/FXP(NX), FXM(NX), FYP(NY), FYM(NY)

1/FLUPR/URFVIS, VISCOS, DENSIT, PRANDT, DEN (NX, NY) , VIS (NX, NY)

$1 /$ COEF / AP (NX,NY), AN (NX,NY), AS (NX,NY), AE (NX,NY), AW (NX,NY), SU (NX,NY), $1 \quad S P(N X, N Y)$

1/TURB/GEN (NX, NY) , CD , CMU, C1, C2 , CAPPA, ELOG , PRED , PRTE , PRM

1/WALLF/YPLUSN (NX), XPLUSW(NY), TAUN (NX), TAUW (NY)

$1 / \operatorname{SUSP} / \operatorname{SUKD}(N X, N Y), \operatorname{SPKD}(N X, N Y)$

$1 / V A R / U(N X, N Y), V(N X, N Y), P(N X, N Y), P P(N X, N Y), T E(N X, N Y), E D(N X, N Y)$

1/KASE T1/UIN, TEIN, EDIN, FLOWIN, ALAMDA,

RSMALL , RLARGE, AL1, AL2, JSTEP, ISTEP, JSTP1, JSTM1, ISTP1, ISTM1

C

$\begin{array}{lllllllllllllll}\text { CHAPTER } & 1 & 1 & 1 & 1 & 1 & 1 & \text { ASSEMBLY OF COEFFICIENTS } & 1 & 1 & 1 & 1 & 1\end{array}$ 1

C

DO $100 \quad I=2$, NIMI

DO $101 \mathrm{~J}=2$, NJM1

C-----COMPUTE AREAS AND VOLUME

$\operatorname{AREAN}=\operatorname{RV}(J+1) * \operatorname{SEW}(I)$

AREAS $=R V(J)$ REW $(I)$

AREAEW $=R C V(J+1) * \operatorname{SNS}(J)$

VOL $=R C V(J+1) \star S N S(J) \star S E W(I)$

C----CALCULATE CONVECTION COEFFICIENTS

$\operatorname{GN}=0.5 *(\operatorname{DEN}(I, J)+\operatorname{DEN}(I, J+1)) * V(I, J+1)$

$G S=0.5 *(D E N(I, J)+D E N(I, J-1)) * V(I, J)$

$\operatorname{GE}=0.5 *(\operatorname{DEN}(I, J)+\operatorname{DEN}(I+1, J)) \star U(I+1, J)$

$G W=0.5 *(\operatorname{DEN}(I, J)+\operatorname{DEN}(I-1, J)) \star U(I, J)$

$C N=G N \star A R E A N$ 


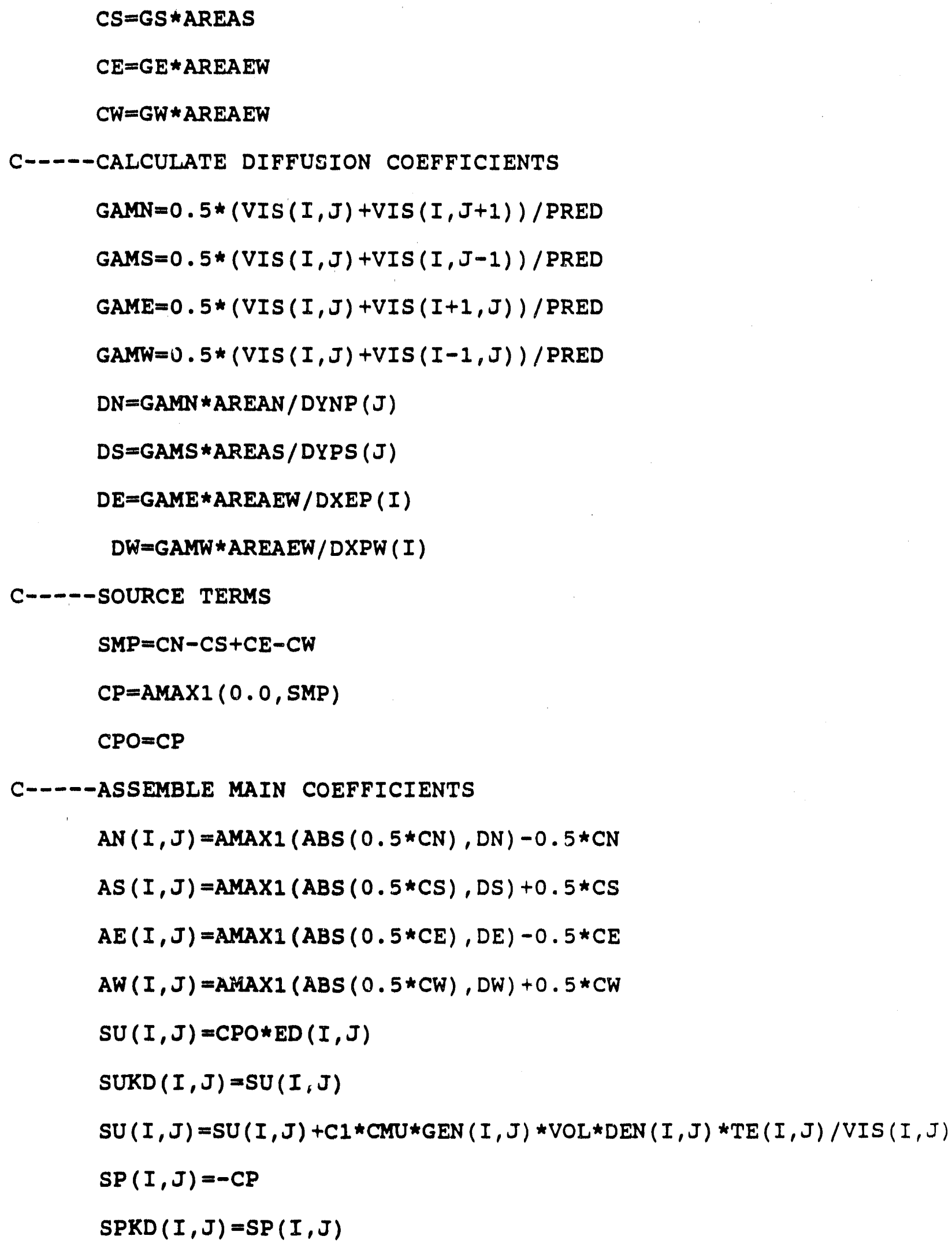




$$
\operatorname{SP}(I, J)=\operatorname{SP}(I, J)-C 2 * D E N(I, J) \star \operatorname{ED}(I, J) \star \operatorname{VOL} / T E(I, J)
$$

101 CONTINUE

100 CONTINUE

C

$\begin{array}{lllllllllllllll}\text { CHAPTER } & 2 & 2 & 2 & 2 & 2 & 2 & \text { PROBLEM MODIFICATIONS } & 2 & 2 & 2 & 2 & 2 & 2\end{array}$ C

CALL MODED

C

CAAPTER 3 FINAL COEFFICIENT ASSEMBIY AND RESIDUAL SOURCE CALCULATION 3

C

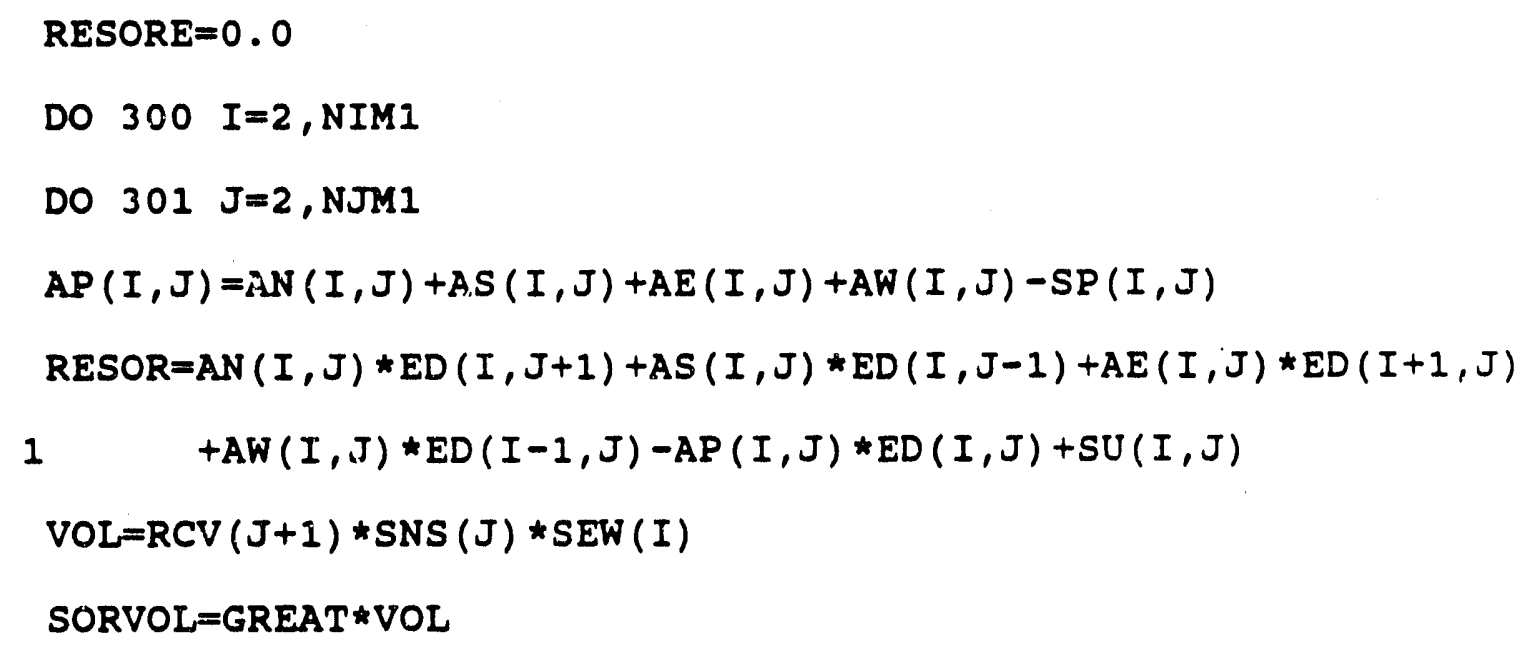




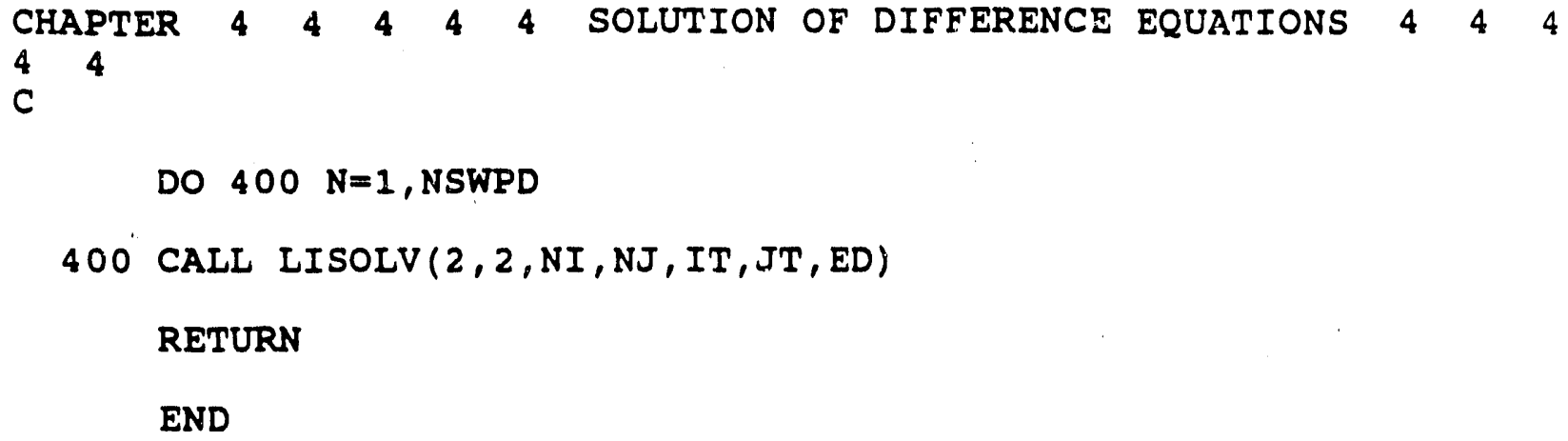



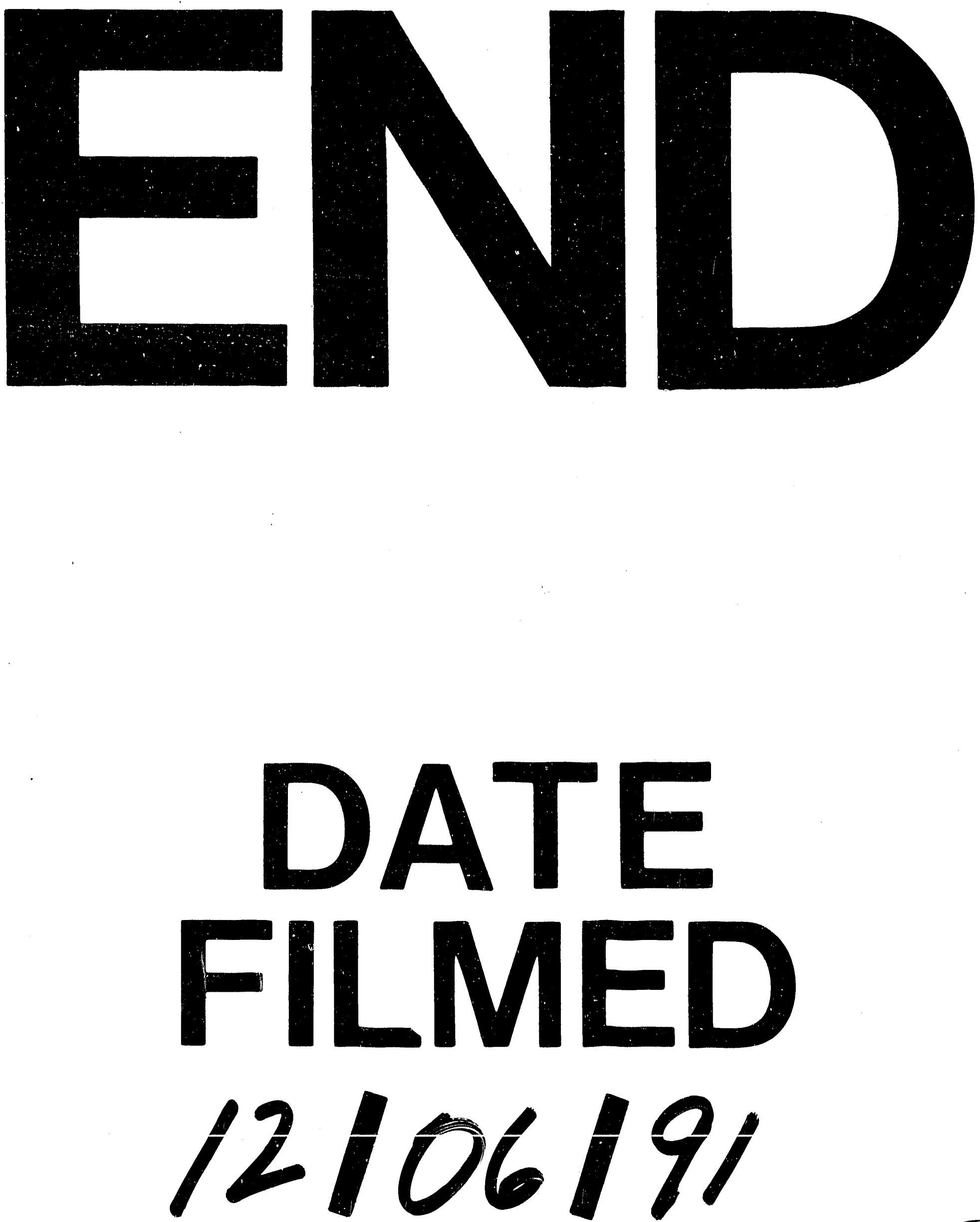

1 
